\title{
Measurement of the Electron Antineutrino Mass from the Beta Spectrum of Gaseous Tritium
}

\author{
David Andrew Knapp
}

\section{DISCLAIMER}

This report was prepared as an account of work sponsored by an agency of the United States Government. Neither the United States Government nor any agency thereof, nor any of their employees, makes any warranty, express or implied, or assumes any legal liability or responsibility for the accuracy, completeness, or usefulness of any information, apparatus, product, or process disclosed, or represents that its use would not infringe privately owned rights. Reference herein to any specific commercial product, process, or service by trade name, trademark, manufacturer, or otherwise does nol necessarily constitute or imply its endorsement, recommendation, or favoring by the United States Government or any agency th $-\mathrm{f}$. The views and opinions of authors expressed herein do not necessarily state or refs. vose of the United States Government or any agency thereof. 


\section{Measurement of the Electron Antineutrino Mass \\ From the Beta Spectrum of Gaseous Tritium \\ David Andrew Knapp}

\section{Abstract:}

A measurement has been made of the mass of the electron antineutrino using the beta spectrum from a source of gaseous molecular tritium, and an upper limit of $36 \mathrm{eV} / \mathrm{c}^{2}$ has been set on this mass. This measurement is the first upper. Iimit on neutrino mass that does not rely on assumptions about the atomic configuration after the beta decay, and it has significantly smaller systematic errors associated with it than do previous measurements.

The mass of the electron antineutrino manifests itself as a small decrease in the available phase space for beta particles emerging from a nucleus. This change in the phase space is observable as a small change in the shape of the beta spectrum of that nucleus. Tritium, with its simple nuclear structure, short half-life, and low decay energy, is the ideal nucleus to use for these measurements. Corrections to the spectrum must be made for the atomic final-state effects, the experimental resolution, and energy loss in the source. The intensity as a function of energy for electrons with kinetic energies near the endpoint of the tritium beta spectrum (18.6 keV) has been measured by extracting the electrons from a low-pressure gaseous source of tritium into an iron-free toroidal-fieid beta spectrometer with an energy resolution of $37 \mathrm{eV}$ FWHA. The data are normalized to the activity in 
the source, and the neutrino mass is determined by fitting the observed spectrum to a theoretical spectrum using a maximum likelihood estimator. The apparatus for this measurement corsists of a gaseous source, a differential pumping restriction, a nonadiabatic magnetic electron focusing system, and a toroidal-field beta spectrometer. The electrons emitted from tritium gas in the source are extracted from it by means of a solenoidal magnetic field, and then accelerated to an energy of $26 \mathrm{keV}$. They are then transported through the differential pumping region and focused through a collimator, which defines the source of the spectrometer. After transport through the spectrometer, they are detected in a position-sensitive resistive-wire proportional counter. The tritium gas itself is recirculated through the source, so. that only a small inventory is required.

Measurements using this apparatus continue, and an eventual sensitivity to a neutrino mass on the order of $10 \mathrm{eV} / \mathrm{c}^{2}$ is expected. 


\section{Contents}

Abstract . . . . . . . . . . . . . . . . . . . . . . . . .

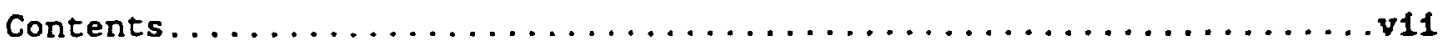

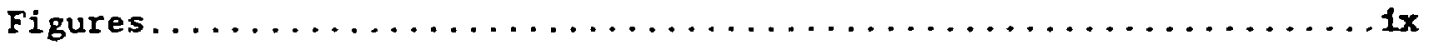

Tables......................................

Chapter 1: Introduction. .......................

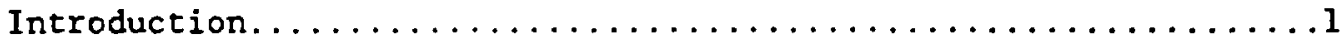

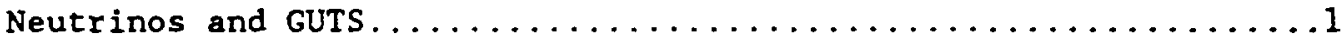

Neutrinos and Cosmology $\ldots \ldots \ldots \ldots \ldots \ldots \ldots \ldots \ldots \ldots \ldots \ldots \ldots \ldots \ldots \ldots \ldots$

Experimental Evidence for Neutrino Mass.................4

The Current Experiment........................5

Chapter 2: History and Theory.....................6

The Fermi Theory of Beta Decay.....................6

The Discovery and Measurement of the Beta Spectrum of Tritium...14 Modern Measurements of the Tritium Beta Spectrum..........17

Experimental Corsiderations in Tritium Beta Decay Experiments....19

Measurements of the Q-Value in Tritium Beta Decay..........27

Methods of Measuring the Tritium Beta Spectrum...........27

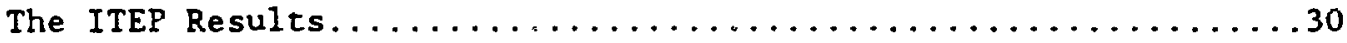

New Results from Tritium Beta Decay....................... 35

Other Experiments to Determine the Neutrino Mass............36

Chapter 3: The Current Experiment.....................44

Difficulties with Solid Sources.....................44

Advantages of Gaseous Tritium Sources.................47

Design Considerations for the Los Alamos Experiment.........48

Design of the Los Alamos Experiment.................51

Chapter 4: Apparatus. . . . . . . . . . . . . . . . . . . 54

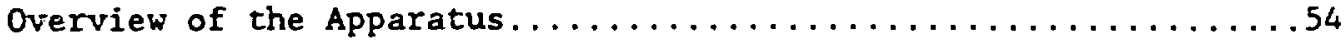

Determination of Source Tube Material.................56

The Gasecus Tritium Source......................61

The Pumping Restriction and Extraction Region..............69

The Beta Spectrometer....................... 76

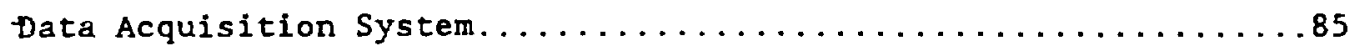


Calculated and Measured Characteristics of the Apparatus......887

Pumping Restriction. ........................

Extraction..............................

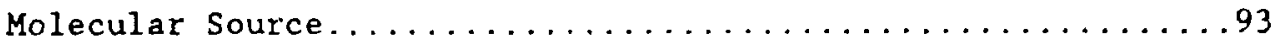

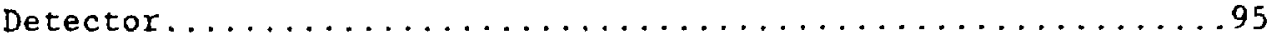

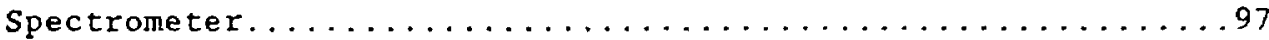

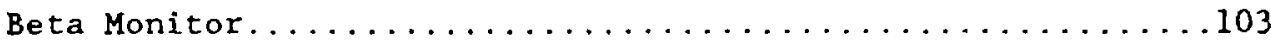

Chapter 5: Data Acquisition, Reduction, and Analysis............ 108 Overview of the Acquisition and Reduction Procedure......... 108 Overview of the Analysis Procedure.................... 108

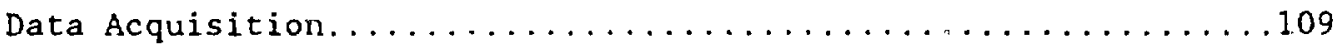

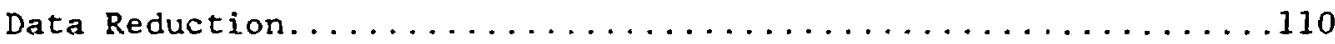

Generation of Theoretical spectra......................113

Fitting Data to the Theoretical Spectrum..................115

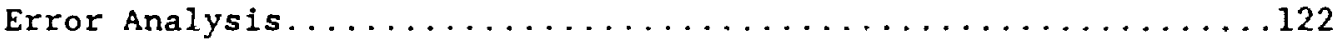

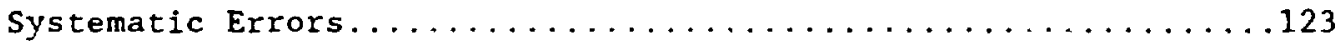

Determination of the Resolution Function...................

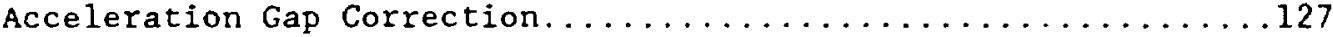

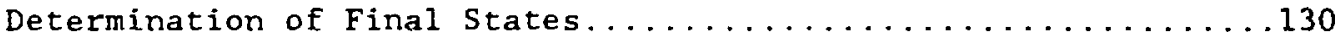

Determination of Scattering in the Source....................

Chapter $6:$ Results................................ 140

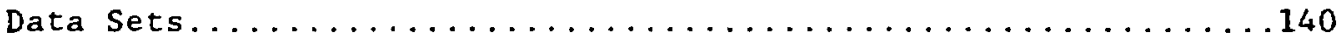

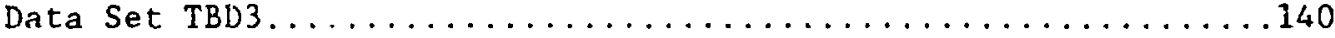

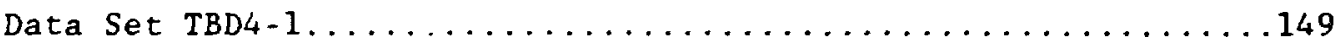

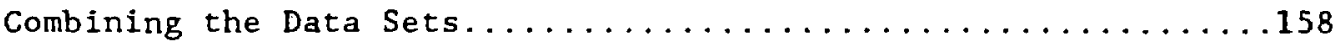

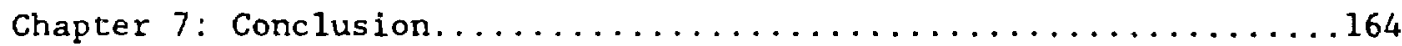

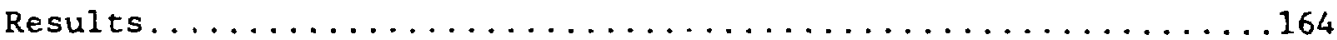

Future Directions for the Experiment.................. 165

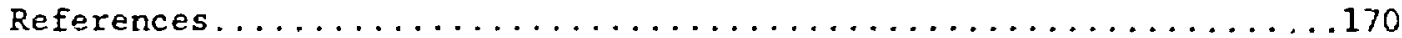

Acknowledgements.................................... 179 


\section{Figures}

Fig. 2.1 Beta Energy Spectrum for Tritium................

Fig. 2.2 Relativistic Unscreened Coulomb Correction for Tritium...8

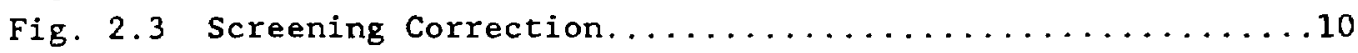

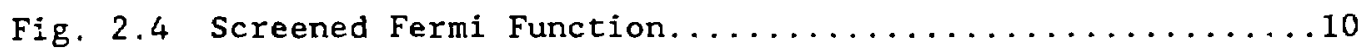

Fig. 2.5 Effect of Corrections to Tritium Beta Spectrum........12

Fig. 2.6 Simpson's Approximation to the Fermi Function........12

Fig. 2.7 Effect of Neutrino Mass on Beta Spectrum of Tritium....13

Fig. 2.8 Summary of Early Tritium Beta Spectrum Results.......21

Fig. 2.9 Final State Distributions for Free Tritium..........23

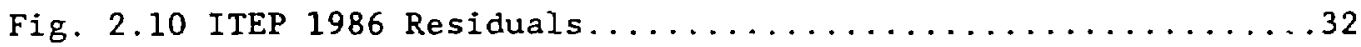

Fig. 2.11 ITEP 1986 Total Resolution Function.............. 32

Fig. 2.12 ITEP 1983 Residuals Analyzed by Bennett........ . . 34

Fig. 2.13 Zurich 1986 Residuals ................... 37

Fig. 2.14 Zurich 1986 Total Resolution Function.............37

Fig. 2.15 Neutrino Oscillation Limits from Reactors..........41

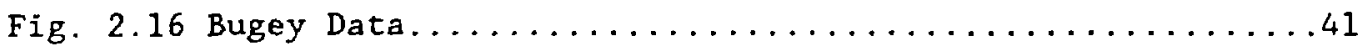

Fig. 3.1 ITEP 1986 Total Resolution Function............46

Fig. 3.2 Schematic of Los Alamos Experiment Functions.........53

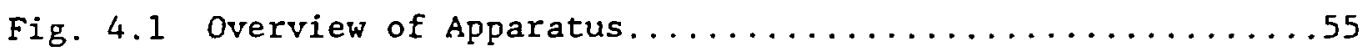

Fig. 4.2 Apparatus used for Recombination Experiments........57

Fig. 4.3 Results of Recombination Experiments...........60

Fig. 4.4 Gaseous Tritium Source..................62

Fig. 4.5 Temperature Control System for the Source...........64

Fig. 4.6 Dissociator and Side Penetration into Source Tube....664

Fig. 4.7 Field Profile of the Superconducting Solenoid.......67

Fig. 4.8 Tritium Gas Handling system...............68

Fig. 4.9 Lọation of Ruthenium Source.................. 70

Fig. 4.10 Pumping Restriction and Extraction Region...........70

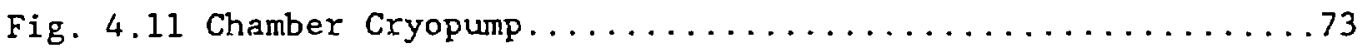

Fig. 4.12 Z-Component of Extraction Field and Electron Orbits ...75

Fig. 4.13 Control Electronics for Extraction Region Magnets..... .77

Fig. 4.14 Geometry of a Toroidal spectrometer...............79

Fig. 4.15 Comparison between Los Alamos and ITEP Spectrometers...79

Fig. 4.16 Detail of the Los Alamos Spectrometer...........80

Fig. 4.17 Active Portion of the Detector...............83

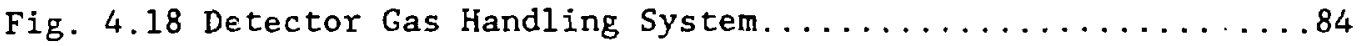

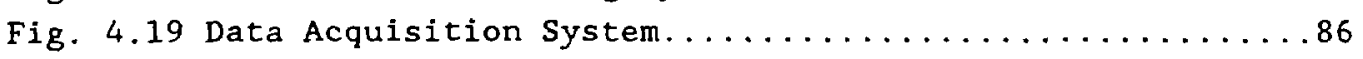


Fig. 4.20 Apparatus used to Measure Pumping Speeds...........88

Fig. 4.21 Apparatus used to Determine Extraction Efficiency,....91

Fig. 4.22 Extraction Efficiency Test Data..............92

Fig. 4.23 Typical Spectra of Spectrometer Detector............98

Fig, 4.24 Resolution Functions Obtained June $1985 \ldots \ldots \ldots \ldots \ldots \ldots 100$

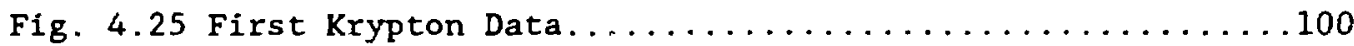

Fig. 4.26 Optimal Electron Gun Resolution................102

Fig. 4.27 Resolution Function at Different Detector Positions...102

Fig. 4.28 Typical Beta Monitor Spectrum.................105

Fig. 5.1 Distribution of Fitted Neutrino Masses for MoCkUP Data.120

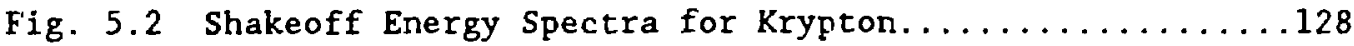

Fig. 5.3 Total Shakeoff Energy Spectrum from Krypton.........128

Fig. 5.4 Final state Spectra for Gaseous Tritium Sources.......132

Fig. 5.5 Energy Loss Spectrum for Singly Scattered Particles...135

Fig. 5.6 Energy Loss Spectrum for Trapped Particles..........135

Fig. 6.1 TBD3 Data........................... 141

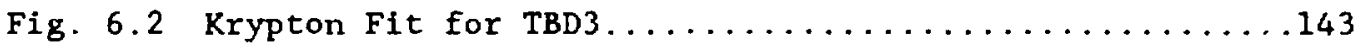

Fig. 6.3 Total Resolution Function for TBD3............146

Fig. 6.4 Residuals for TBD $\ldots \ldots \ldots \ldots \ldots \ldots \ldots \ldots \ldots \ldots \ldots$

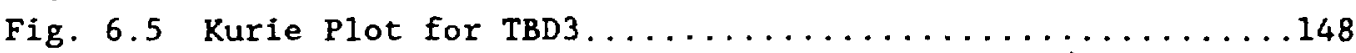

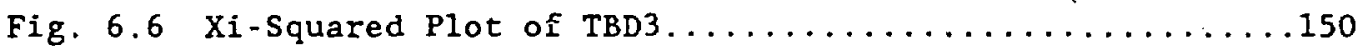

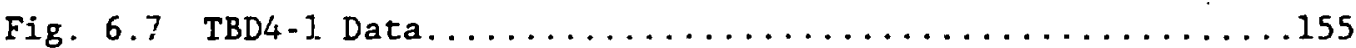

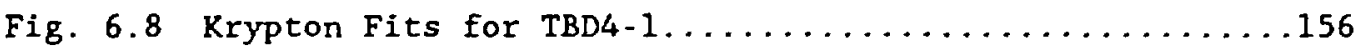

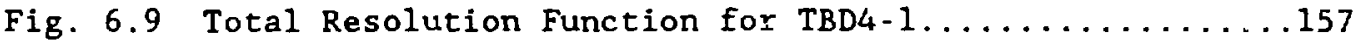

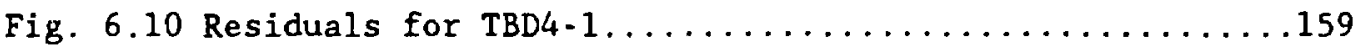

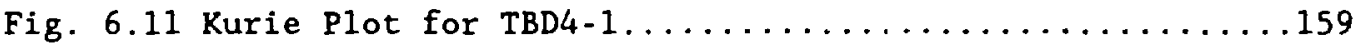

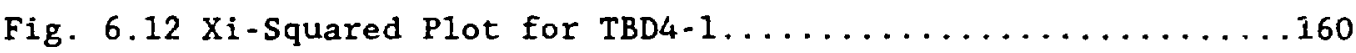

Fig. $6.13 \mathrm{Xi-Squared} \mathrm{Plot} \mathrm{for} \mathrm{Both} \mathrm{Sets} \mathrm{of} \mathrm{Data...........161}$ 


\section{Tables}

Table 2-1 Summary of Early Tritium Results................20

Table 2-2 Sumnary of Q-Value Measurements..............28

Table 5-1 Tests of Variaus Estimators with Random Data.......121

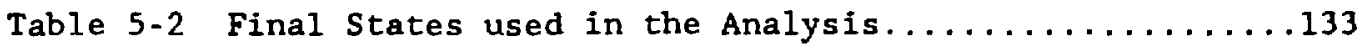

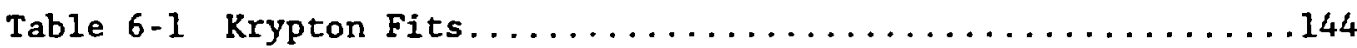

Table 6-2 Data Fits..........................147

Table 6-3 Systematic variation Checks................. 151

Table 6-4 Curvature, Covariance, and Correlation Matrices......152 


\section{Chapter 1:}

Introduction

\section{Introduction}

The possibility of the existence of a rest mass for the electron antineutrino is currently a topic of considerable interest. A nonzero rest mass for the neutrino would be the first indication of physics beyond the "standard model". In addition, the neutrino has been put forward as a possible candidate for the "missing mass" problem in cosmology. Many candidates for observable departures from the "standard model" have not been observed, so attention has been placed on the neutrino as one of the best remaining hopes for discriminating between differing Grand Unification Theories (GUTS). The properties of neutrinos are still not very well understood more than 50 years after their existence was first postulated. A positive value for the mass was reported in 1980 [Lyu80], and a large number of other experiments have been undertaken to check this result.

Neutrinos and GUTS

In the "standard model", the neutrino is constrained to be completely left-handed and to have zero mass as an initial condition. There is no compelling theoretical reason, however, for the mass to be zero; indeed, by analogy to the quark sector, one would expect $m_{\nu} \approx m_{\ell}$, where $\ell$ is the lepton associated with the particular neutrino. In fact, for the electron and electron neutrino, $\mathrm{m}_{\mathrm{e}} / \mathrm{m}_{\nu}>10^{4}$. In general, 
however, grand unified theories (GUTS) predict nonzero neutrino masses. Therefore, the neutrino masses pose one of the most interesting unsolved problems in theoretical physics [Ros86]. One way of generating small neutrino masses is by the "see-saw" mechanism of GeIl-Mann, Ramond, and Slansky [Ge179]. It requires that neutrinos be Majorana particles, that is, that they be self-conjugate. Neutrinos, since they have no charge, could be self-conjugate, unlike the other leptons, which must be Dirac particles. However, a Majorana mass for the neutrino implies that lepton number cannot be strictly conserved. In the "see-saw" scheme, a large mass term for right-handed neutrinos creates a small left-handed Majorana mass through a Dirac mass term which mixes the right-and lefthanded fields. Clearly, this model predicts lepton number violation. This idea was extended by witten [Wit80], who showed that in the minimal $O(10)$ theory, neutrinos would naturally acquire a mass of $-1 \mathrm{eV}$. Neutrinos can acquire a natural Dirac mass in left-right symmetric theories [Cea84] through radiative corrections. Indeed, a model has been proposed to generate a Dirac mass in the $S U(2) \times U(1)$ and $S U(5)$ models [Ron83]. This model introduces a right-handed reutrino and a new symmetry for that neutrino, which couples it to the left-handed neutrinos. The mass of the electron neutrino is predicted to be about $1-10 \mathrm{eV}$.

The question of whether neutrinos are Majorana or Dirac particles remains unsolved. As will be seen later, different experiments are sensitive to this question. In order to differentiate between theories, some idea of the magnitude of the neutrino mass would be halpful. So a variety of experiments sensitive to different kinds of neutrino masses seems indicated. 


\section{Neutrinos and Cosmology}

A tantalizing hint of physics beyond the "standard model" is provided by the "missing mass" problem in cosmology. This problem has been a topic of considerable interest in recent years. The average density of the Universe can be characterized by the parameter $\Omega=\rho / \rho_{C}$, where $\rho_{c}$ is the critical density required to close the Universe. That is, if $\Omega>1$, the Universe is closed and will eventually collapse; if $\Omega<1$ it will expand indefinitely; and if $\Omega=1$ it is "flat" and will expand forever but come to rest at $t=\infty$. The observed mass from the light emitted by galaxies gives an upper limit on baryonic mass such that $\Omega_{B}<0.1$ [Fra82]. Galculations of primordial nucleosynthesis provide an upper limit for the total possible baryonic density: $\Omega_{B} \leq 0.14-0.19$ [Yan84]. If $\Omega<1$, then it varies with time as $\Omega-t^{-1}$. with a characteristic time scale of the Planck time $t_{P}=5 \times 10^{-44} \mathrm{sec}$. [Fra82]. Therefore, if $\Omega=0.1$ at the present, then $1-\Omega$ would have been about $10^{-60}$ at $t=t_{P}$. It is more appealing to have $\Omega$ exactly 1 . Indeed, new "inflationary" theories of the early evolution of the Universe require that $\Omega=1$ [Gut81]. If this is indeed the case, then 80-908 of the mass of the Universe is made up of nonbaryonic matter. The neutrino is the only particle known to exist that could provide this mass. The neutrino mass required for closure would be $10^{\prime} \mathrm{s}$ of $\mathrm{eV}$. An early upper limit on the mass of $8 \mathrm{eV}$ was calculated by Cowsik and McClelland [Cow72] based on a value of $50 \mathrm{~km} \mathrm{sec}-1 \mathrm{Mpc}^{-1}$ for the Hubble constant and an assumption that there were two kinds of neutrinos, each of which had an antiparticle and two spin states. This limit seems too extreme when compared to newer calculations, but it generated interest in neutrinos as possible contributors to the mass of the Universe. The 
calculations of Schramm and Steigman [Sch81] imply that the summation of masses of stable neutrinos $\sum_{i} m_{\nu}<100 \mathrm{eV}$ for Majorana neutrinos and $\sum_{i} m_{\nu}<50 \mathrm{eV}$ if the neutrinos are Dirac particles.

The missing mass problem extends down to smaller scales as well. Rotation curves of galaxies indicate the presence of nonluminous halos surrounding them [Rub83]. Cluster dynamics indicate that the galaxies contained in them are more massive than could be accounted for by ordinary matter [Fab79]. Neutrinos have been proposed as a candidate for this missing mass [Har82, Rub83], but they are considered by some theorists to be too energetic to be gravitationally bound [Kra84]. It is possible, of course, that the halos around the galaxies are nonluminous baryonic matter.

Other objections have been raised about the suitability of a neutrino dominated universe, based on models of galaxy formation [Dav81, Kai83, Hut84]. The conclusion of Hut and White [Hut84] was that a neutrino dominated universe would require either two species of massive neutrinos, the heavier of which would be unstable, or the addition of new particles which would be responsible for galaxy formation.

\section{Experimental Evidence for Neutrino Mass}

The final impetus for the measurement of the neutrino mass comes from interesting experimental results. In 1980, when the experiment described herein was initiated, there were several puzzling experimental results outstanding. The first was the measurement of the solar neutrino flux by Davis et a1. [Row84]. This flux has consistently come out to be about $1 / 3$ the expected flux from astrophysical calculations [Bah84]. The problem posed by this discrepancy has not yet been solved. 
Neutrino oscillations had been reported by Reines [Rei80], and, though this result was later negated, it served to spark the field. Also in 1980, a positive result for the electron antineutrino mass $\left(m_{\nu} \approx 30 \mathrm{eV}\right)$ was reported in a tritium beta decay experiment [Lyu80]. This experiment will be treated in more detail in Chapter 2 . It has not yet, however, been refuted.

The Current Experiment

The experiment described in this thesis is an attempt to resolve some of the outstanding issues about neutrino masses. A neutrino mass on the order of $10 \mathrm{eV}$ is a very difficult quantity to measure. Atomic electron effects and solid-state effects have similar energies associated with them, and, unless they are extremely well quantified, they can easily overwhelm a small neutrino mass. To minimize the uncertainties inherent in this kind of measurement, an apparatus has been constructed at Los Alamos to measure the beta spectra of gaseous tritium sources. The use of a gaseous source assures that the small effects which can interfere with a measurement of the neutrino mass are minimized and well understood. Data will be presented on the spectrum of molecular tritium, and an upper limit on the electron antineutrino mass derived. 
Chapter 2:

History and Theory

The Fermi Theory of Beta Decay

The neutrino was first postulated by Pauli in 1930 to account for the electron energy spectrum of beta decay. In a typical beta decay process:

$$
{ }^{\wedge} \mathrm{Z} \rightarrow{ }^{\wedge}(\mathrm{Z}+1)+\mathrm{e}^{-}+\bar{\nu}_{\mathrm{e}}
$$

the energy spectrum of the beta particles is a continuum, which would violate energy conservation if there were no third body to take away the excess momentum and energy. Interestingly, the neutrino was first known as the "neutron"; the particle which we now call the neutron had not yet been discovered [Pei83]. In 1934 Fermi published his famous theory of beta decay, of which the neutrino was an important part [Fer34]. He pointed out that the rest mass of the neutrino would affect the energy spectrum of electrons given off in beta decay, and gave the general form for the spectral shape in the presence of a nonzero neutrino rest mass.

The Fermi form for the probability of emitting an electron with total energy $E$ is given by:

$$
d N(E)=K|M|^{2} F(Z, R, E) P_{e} E\left(E_{0}-E\right)\left[\left(E_{0}-E\right)^{2}-m_{\nu}{ }^{2} c^{4}\right]^{1 / 2} d E,
$$

where $K=G_{F}^{2}\left(m^{5} c^{4} / 2 \pi^{3} h^{7}\right) \cos ^{2} \theta_{c}, M$ is the nuclear matrix element for the transition (for tritium $|M|^{2} \approx 5.55$ ), $P_{e}$ is the electron momentum, $E_{0}$ is 
the endpoint energy (the maximum electron energy for zero neutrino mass), and $m_{\nu}$ is the neutrino mass. The shape of this function is shown in Fig. 2.1.

$F(Z, R, E)$, known as the Fermi function, is a Coulomb correction term that results from the effect of the nuclear charge on the wavefunction of the emitted electron. The function is just the ratio of the electron wavefunction at the nuclear radius with the coulomb field on to that with it off. For positrons, it tends to decrease the probability of emission, and for electrons, it enhances it. In both cases the effect is greatest at low beta energies. Indeed, it produces a finite probability of emission of an electron of zero kinetic energy. In a nonrelativistic model with a point nucleus it is given by:

$$
F_{N}(Z, E)=y / \mid 1-e^{-y_{1}}
$$

where $y=2 \pi Z \alpha /\left(v_{e} / c\right)$. A more sophisticated model [NBS51] that includes relativistic wavefunctions and the nuclear size gives the result:

$$
F(Z, R, E)=2(S+2)\left(2 P e^{m} e^{c^{2}} \frac{R}{\pi c}\right)^{2 S} e^{\pi \delta} \frac{|\Gamma(1+S+i \delta)|^{2}}{|\Gamma(3+2 S)|^{2}}
$$

where $S=\left[1-(Z \alpha)^{2}\right]^{1 / 2}-1, Z$ is the charge of the daughter nucleus, $R$ is the nuclear radius, $\delta=Z \alpha /\left(\mathrm{v}_{e} / \mathrm{c}\right)$, and $\mathrm{p}_{e}$ is the electron momentum in units of $\mathrm{m}_{e} \mathrm{c}$. For tritium $(\mathrm{z}-2)$ beta particles emitted at $18 \mathrm{keV}$, $F(Z, R, E)$ is about 1.18. A plot of $F(Z, R, E)$ for tritium is shown in Fig. 2.2 .

In 1936, Rose proposed that the atomic electrons would screen the nuclear charge from the outgoing beta particle, thereby changing the 


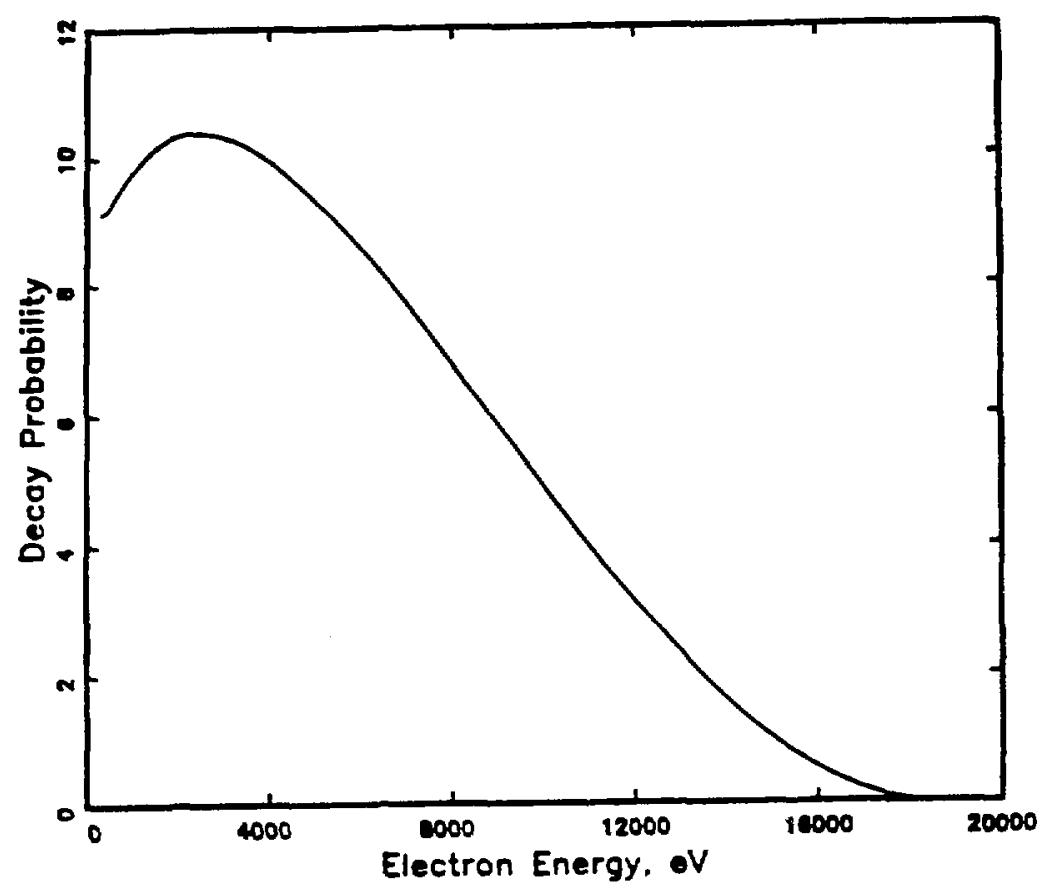

F1g. 2.1. The beta energy spectrum for tritium. Amplitude is in arbitrary units.

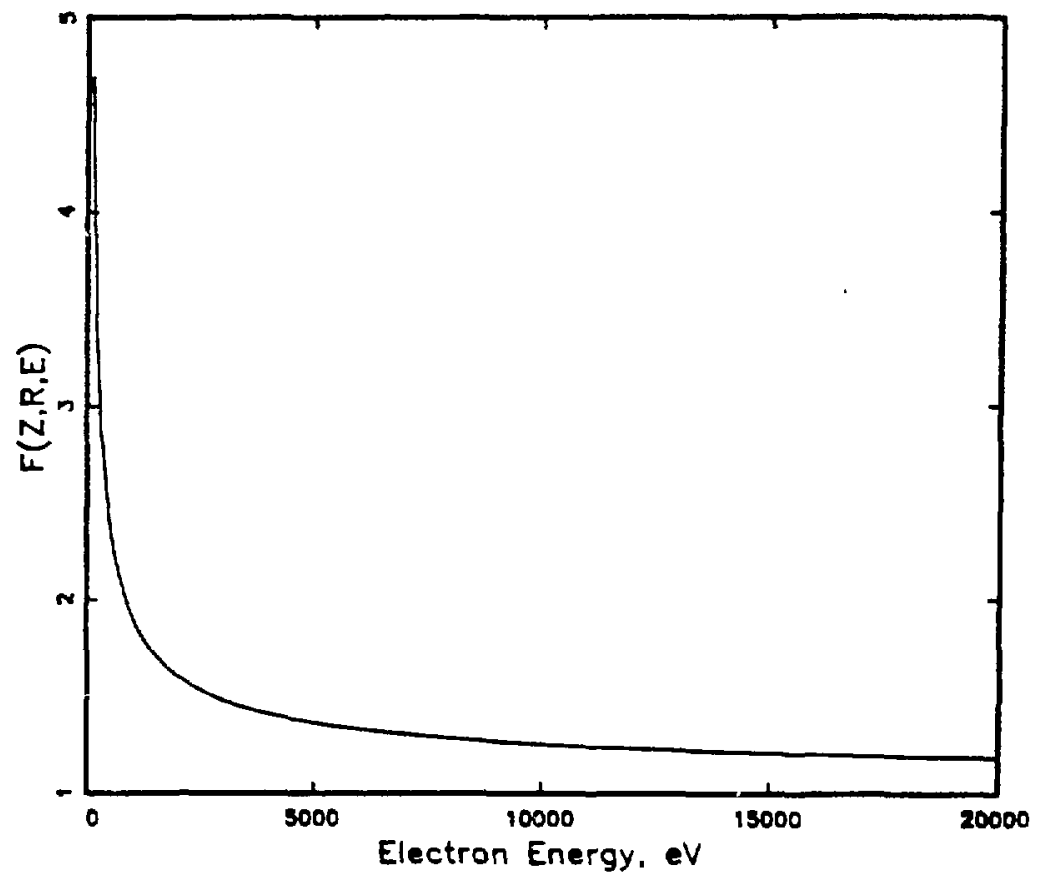

Fig. 2.2. Relativistic unscreened Coulomb correction factor $F(Z, R, E)$ for tritium. 
form of the Coulomb correction factor [Ros36]. Using a W.K.B. approximation for the outgoing wavefunction, he derived a new formula for the factor:

$$
F^{\prime}(Z, R, E)=F\left(Z, R, E-V_{0}\right)\left[\left(E-V_{0}\right) / E\right]
$$

Where $E$ is the total energy of the electron and $V_{0}$ is the Thomas-Fermi potential at the nuclear radius, given by:

$$
v_{0}=1.45 z^{4 / 3} \alpha^{2} m_{e}
$$

In this case $z$ is the charge of the parent nucleus. The ratio between the unscreened and screened spectra is shown in Fig. 2.3. The magnitude of this correction is very small at higher electron energies, but becomes significant for electrons with kinetic energy less than $2 \mathrm{keV}$. A plot of the screened Coulomb corrections is shown in Fig. 2.4, and the effect on the beta spectrum is shown in Fig. 2.5.

Longmire and Brown re-derived a similar expression in 1949 [Lon49], also using the W.K.B. method, and assuming that the effect of screening is constant over the volume between the nuclear radius and the radius of the atom. Their expression is:

$$
F^{\prime}(Z, R, E)=F\left(Z, R, E-V_{0}\right)\left[\left(E-m_{e} c^{2}\right) /\left(E-m_{e} c^{2}-v_{0}\right)\right]^{1 / 2}
$$

The difference between these two expressions is interesting but negligible above about $I \mathrm{keV}$ for light nuclei. It is interesting to note that both approximations break down at $\mathrm{E}-\mathrm{m}_{\mathrm{e}} \mathrm{c}^{2}=\mathrm{v}_{0}$, predicting an infinite probability of decay. 


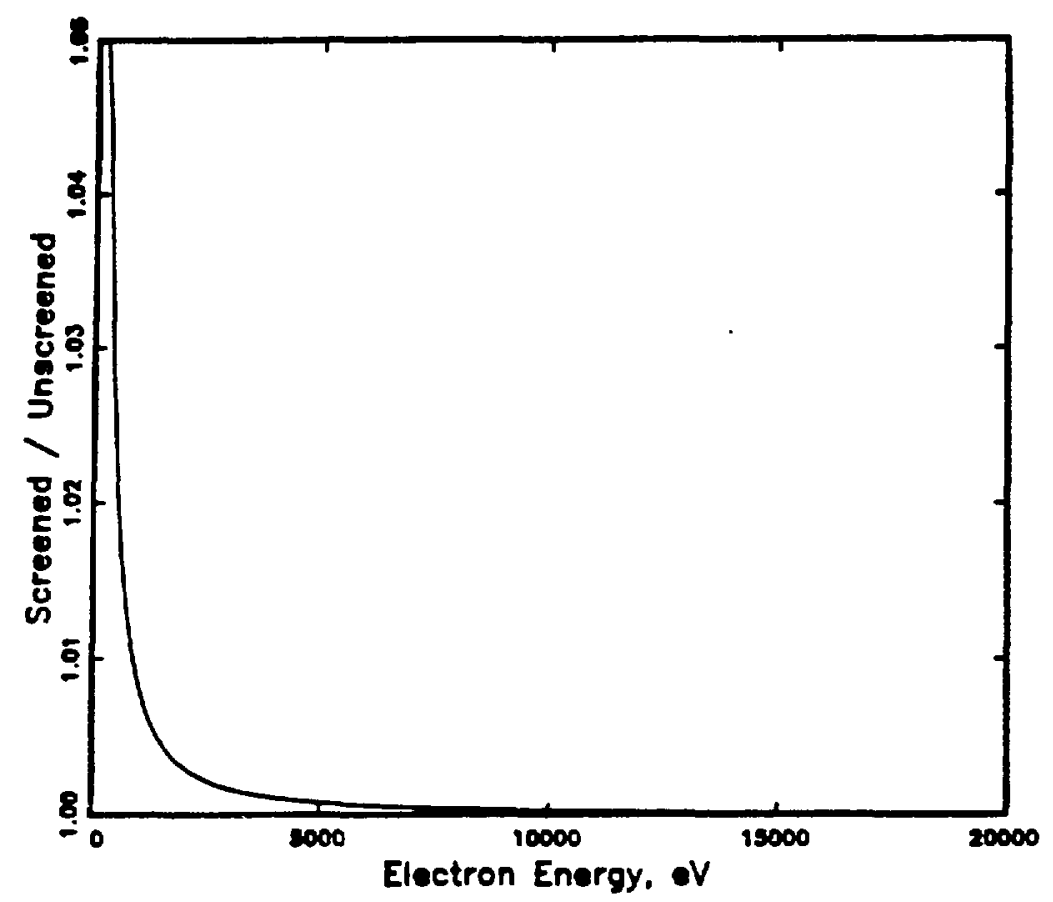

Fig. 2.3. The screening correction of Rose [Ros36].

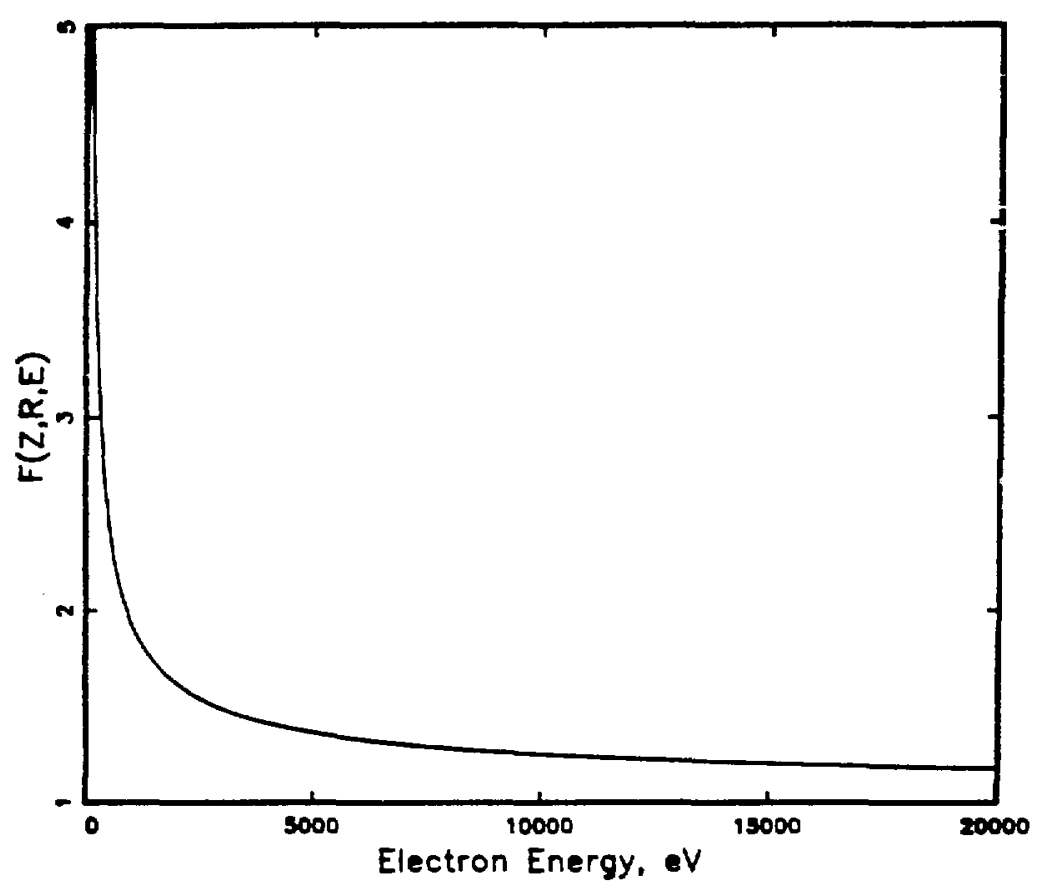

Fig. 2.4. The screened Fermi function $F^{\prime}(2, R, E)$. 
Simpson [Sim81] stated that for tritium, the relativistic Fermi function, including the screening corrections of Rose, can be approximated by an expansion of the nonrelativistic one without screening corrections:

$$
F(Z, R, E) \propto F_{N}(Z, E)\left(1.002037-0.001427 v_{e} / c\right)
$$

This approximation is good to better than 0.058 for most of the spectrum, excluding only extremely low energy electrons (see Fig. 2.6).

Kurie showed that the Fermi form for the beta spectrum could be linearized by plotting $\left[N(E) / E p_{e} F(Z, R, E)\right]^{1 / 2}$ vs. $E$ [Kur36]. This parameterization allows the effect of neutrino mass to be seen much more clearly, as is shown in Fig. 2.7. In addition, it allows the endpoint energy to be extrapolated from data taken at lower energies, where the count rates tend to be higher. This extrapolated endpoint is independent of the neutrino mass. It turns out that the spectrum of tritium has an extremely straight Kurie plot, since the transition is allowed and the nuclear effects are small.

Many of the first beta spectra that were measured were composite spectra: that is, they contained transitions which leave the nucleus in various excited states, so the shapes did not agree well with the predictions of the Fermi theory [Pei83, Bet39]. In addition, some of these spectra involved forbidden transitions, in which the matrix elements for the decays can depend on energy. This prompted some theoretical speculation and alternative forms for the spectral shape were proposed [Kon35]. However, as is well known, the Fermi theory has withstood the test of time. 


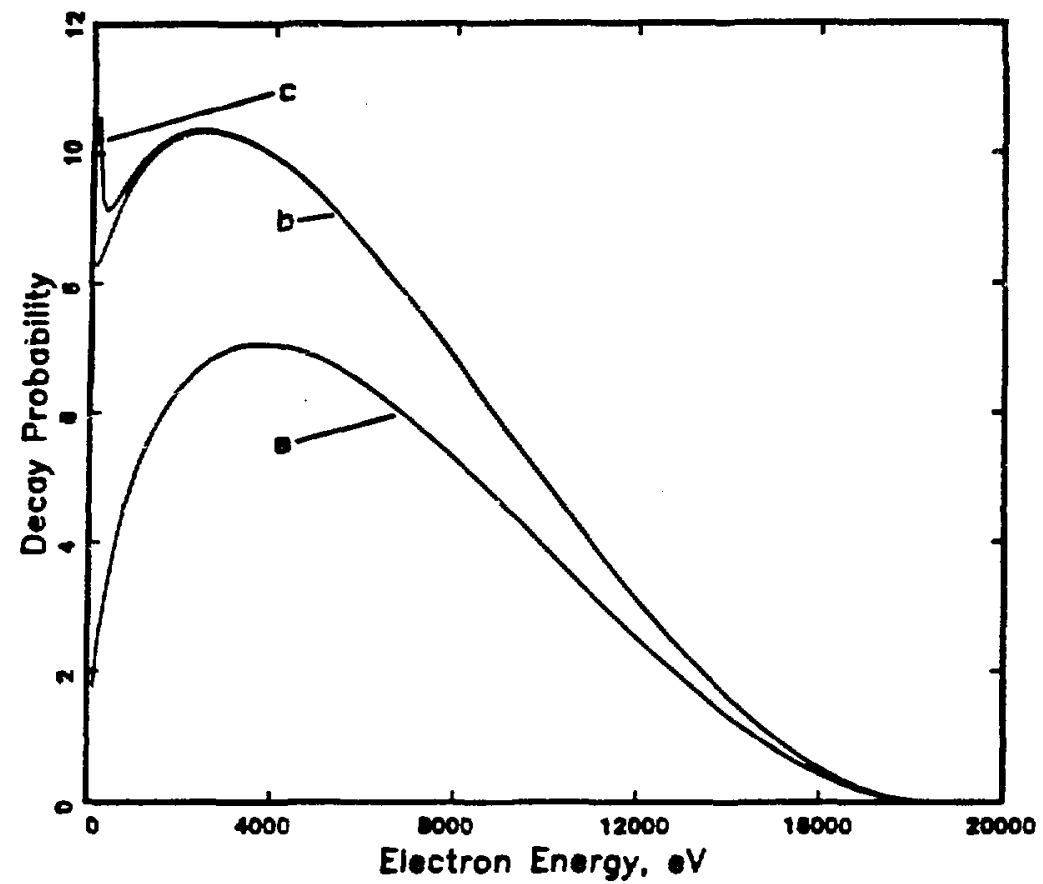

Fig. 2.5. Effect of various corrections to the beta spectrum of tritium: a) uncorrected spectrum, b) with relativistic unscreened Fermi function, c) with relativistic screened Fermi function.

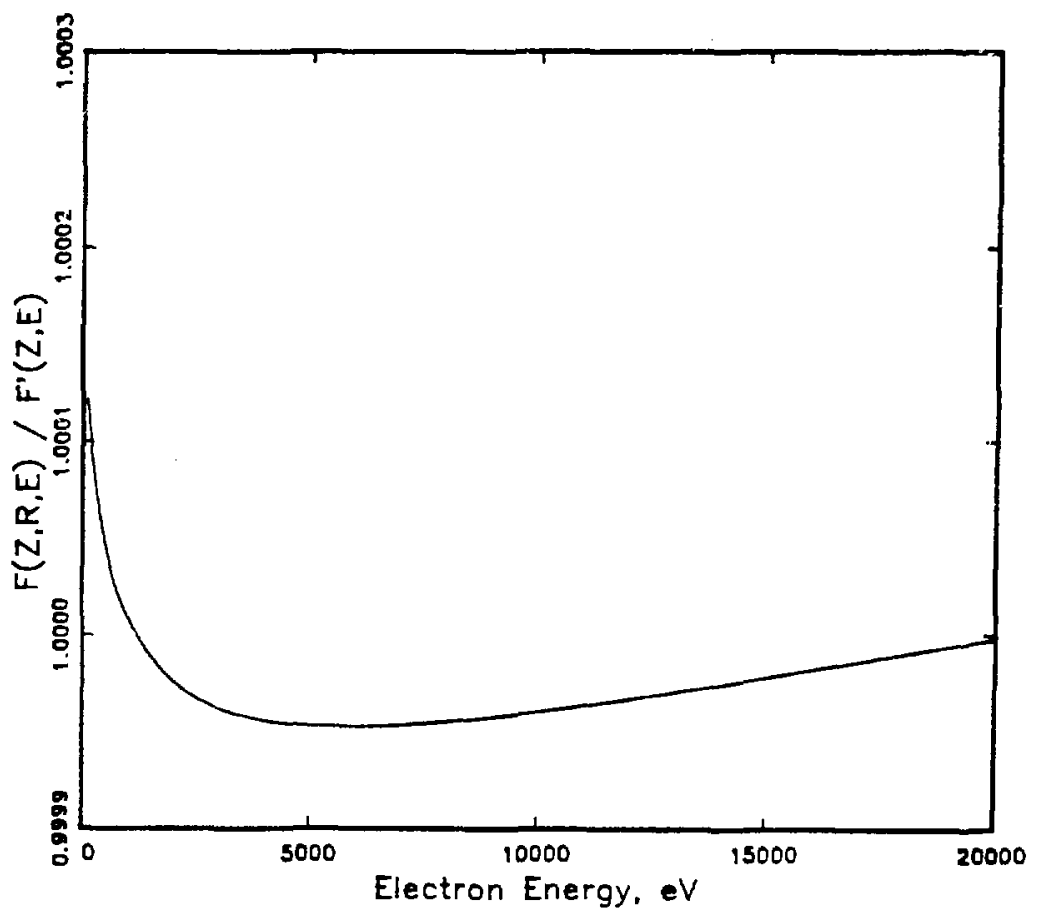

Fig. 2.6. Simpson's approximation to the screened relativistic Fermi function. $F^{\prime}(Z, E)$ is the approximation; $F(Z, R, E)$ is the true value. 
a)
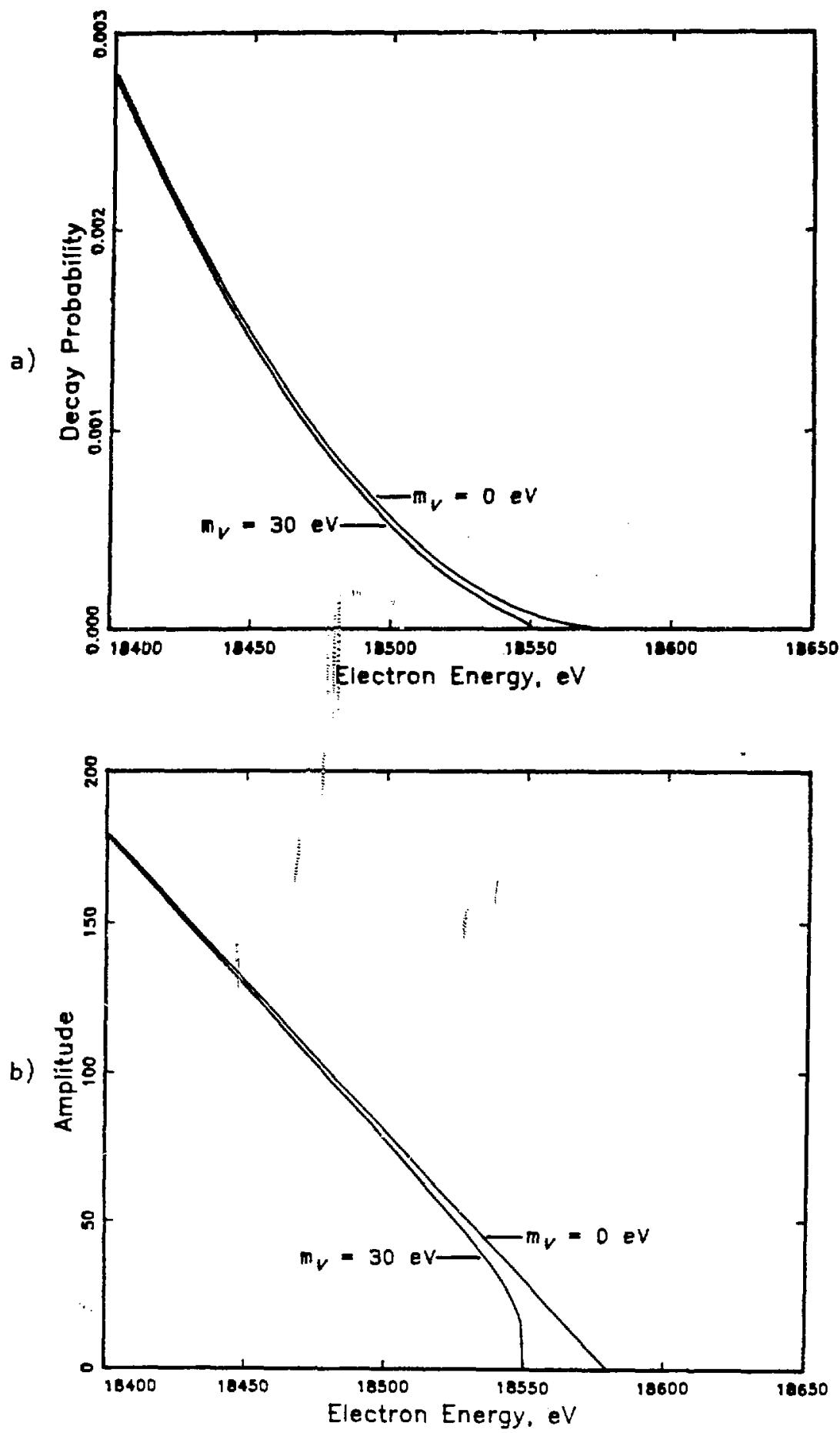

Fig. 2.7. Effect of neutrino mass on the beta spectrum of tritium: a) actual beta spectrum, b) Kurie plot. 
The Discovery and Measurement of the Beta Spectrum of Tritium

The beta spectrum of tritium has proven best for the measurement of the electron antineutrino mass because of its low endpoint energy, short half-life, and straight Kurie plot. The low endpoint energy maximizes the effect of the neutrino mass on the phase space factors. The decay is superallowed, which means that the half-life is short for the energy released. The straight kurie plot allows an accurate endpoint extrapolation which in turn pernits extremely high precision to be obtained for the value of the neutrino mass.

Beta rays from tritium were first observed in 1938 by Libby and Lee in the course of performance tests of a new low-energy beta detector [Lib39]. The detector consisted of a sćreen-wall Geiger counter which detected any particles entering the wall. A nagnetic fièld was varied. until no particles were seen to enter the detector, thus determining the maximum particle energy. Unfortunately, they did not recognize that the extremely soft (endpoint was given as $13 \pm 5 \mathrm{keV}$ ) electrons emitted from a Be target that had been irradiated with deuterons were from tritium. They initially attributed the radiation to ${ }^{10} \mathrm{Be}$.

In 1939 Alvarez and Cornog [Alv39a] first reported the discovery of radiation from ${ }^{3} \mathrm{H}$. They were able to show that the activity was an isotope of hydrogen by passing it through a cryogenic charcoal bed and diffusing it through palladium. Later [Alv40a] they reported a halflife of $150 \pm 40$ days. This value was reported to be in error because of diffusion of the tritium through a rubber tube connected to the first apparatus used to contain the tritium, and the new half-life was 
reported to be too long to measure by watching the activity decay over a period of months [Alv40b].

Meanwhile, 0 Neal and Goldhaber realized that the soft beta rays from Libby and Lee's beryllium bombarded with deuterons might be coming from ${ }^{3} \mathrm{H}$ [One40a]. They tested this hypothesis by precipitating the beryllium from a sample, and found that the activity had disappeared. They found a maximum energy of $15 \pm 3 \mathrm{keV}$, and later measured a halflife of 31.4 years [One400].

The endpoint energy of the beta spectrum of tritium turned out to be exceptionally difficult to measure, probably because of the low intensities that are available at higher energies. It is interesting to note that it was many years before anyone attempted to extrapolate the endpoint of the tritium spectrum by using a Kurie plot. Instead, most of the early efforts concentrated on measuring the highest energy electrons observed from tritium. In 1940, Perlow [Per40] estimated the value to be either 10 or $19 \mathrm{keV}$ from his measurements of the ${ }^{6} \mathrm{Li}+1_{\mathrm{H}} \rightarrow{ }^{3} \mathrm{He}+{ }^{4} \mathrm{He}$ reaction. In 1941 , Brown made the next direct measurement of the maximum energy by using a Geiger counter to measure the range in helium [Bro41] and found an endpoint of $9.5 \pm 2 \mathrm{keV}$.

In 1941 Nielsen measured the maximum range of tritium beta rays in a cloud chamber and observed a maximum energy of $14.5 \pm 1 \mathrm{keV}$ [1rie41]. O'Neal pointed out that the ranges he had observed agreed with those of Brown, the difference in endpoint energies (15 keV vs. $9.5 \mathrm{keV}$ ) arising from different range-energy calibrations [One41]. However, an experiment performed in Los Alamos in 1946 by Watts and Williams [Wat46] 
In which the betas were accelerated through a thin window into a Geiger counter gave an upper limit of $11 \pm 2 \mathrm{keV}$ for the beta energies.

Konopinski [Kon47] was concerned that the half-life of tritium was too short to agree with the measured maximum energies, and postulated a neutrino mass of 11 to $17 \mathrm{keV}$ to explain the discrepancy. Pruett [Pru48] refined this estimate to a mass of between 25 and $150 \mathrm{keV}$, including the Coulomb effects and a relativistic correction. He also pointed out that an energy release of around $20 \mathrm{keV}$ in the tritium would eliminate the mass needed to account for the problem.

Another cloud chamber experiment was done in 1948 by Byatt et al. [Bya48] using a small amount of tritiated water. The longest track length seen led tc an endpoint energy of $11 \pm 1 \mathrm{keV}$, which was revised to $17.0 \mathrm{keV}$ upon further analysis [Bya49]. An experiment measuring the bremsstrahlung from tritium betas stopped in zirconium and tantalum was performed by Graves and Meyer in 1949 [Gra49]. The endpoint was deduced to be $18.0+0 /-.5 \mathrm{keV}$.

The first real measurement of the shape of the tritium spectrum was made by Curran, Angus, and Cockroft in 1948 [Cur48], using a proportional counter. They initially reported that the endpoint was at $16.9 \mathrm{keV}$, and that the spectrum did not fit the Fermi distribution at low energies. In addition, they reported an upper limit on the neutrino mass of $1.7 \mathrm{keV}$. In a more extensive report published later [Cur49a], the endpoint estimate was raised to $17.9 \mathrm{keV}$, and the discrepancies beiween the observed spectrum and the theoretical Fermi spectrum were minimized (the approximation they had originally used to the Fermi distribution was a rather poor one). However, the spectrum seemed to 
fit a neutrino mass of about $1 \mathrm{keV}$. Yet another report was published [Cur49b], in which the endpoint energy was reported to be between 18.0 and $18.6 \mathrm{keV}$ and the neutrino mass (for an $18.0 \mathrm{keV}$ endpoint) to be between 0 and $1 \mathrm{keV}$.

Meanwhile, Hanna and Pontecorvo [Han49] were also engaged in a proportional counter experiment. Their endpoint energy, corrected for resolution effects, was given as $18.9 \mathrm{keV}$. Slack et al. [S1a49] calculated the endpoint energy from average decay energy data obtained by Jenks et al. [Jen49, Jen50]. The new result was $18.6 \pm .2 \mathrm{keV}$, and the problem of the half-life vs. the energy release was resolved. In addition, the problem of deviations from the Fermi distribution was shown to be nonexistent by Cooper and Rogers [Coo50], who compiled all the existing tritium data taken up to that date and showed that they fit the theoretical distribution very well.

Modern Measurements of the Tritium Beta Spectrum

More modern experiments to determine a small neutrino mass from the tritium beta spectrum began with Hamiltor et a1. in 1952 [Ham52]. A hemispherical electrostatic integral spectrometer was used to place an upper limit on neutrino mass at $250 \mathrm{eV}$. This experiment, however, was done with a thick source and the endpoint energy was measured at $19.4 \pm .4 \mathrm{keV}$, enough in error to place the results in some doubt. Also in 1952, Langer and Moffat [Lan52] used a high-resolution magnetic spectrometer to measure the spectrum. They also placed an upper 1 imit of $250 \mathrm{eV}$ on the neutrino mass, and obtained an extrapolated endpoint $(17.95 \pm .10 \mathrm{keV})$ that today seems in error. In 1959, Porter [Por59] 
attempted to resolve the discrepancies in the measured endpoint energies of tritium with another magnetic spectrometer experiment. His endpoint value of $18610 \pm 100 \mathrm{eV}$ was by far the most precise up to that time.

Up to this time, most experiments that were undertaken used standard spectrometers that could be used for other experiments besides tritium beta spectrum measurements, and the experiments measured the shape of most of the tritium spectrum. Salgo and Staub [Sal69] devised the first experimental apparatus specifically optimized for investigations of the beta spectrum of tritiun near the endpoint. Their electrostatic decelerating spectrometer was in many ways similar to those used today. Despite a signal-to-noise ratio of less than $1 / 300$ in the last $1 \mathrm{keV}$ of the spectrum, they were able to set an upper limit of $200 \mathrm{eV}$ ( $80 \mathrm{f}$ C.L.) on the neutrino mass. Their measured endpoint was somewhat higher $(18700 \pm 60 \mathrm{eV})$ than that which is accepted today; however, this would not affect their result significantly.

At about the same time, Daris and St.-Pierre [Dar69] measured the spectrum using a magnetic spectrometer with $47 \mathrm{eV}$ resolution. Their upper limit on neutrino mass was $75 \mathrm{eV}$ (at the 1 sigma leve1), and their endpoint measurement was $18570 \pm 75 \mathrm{eV}$. Both these values were a significant improvement over those existing at that time. An endpoint measurement using a tritium-implanted Si(Li) detector was also undertaken at nearly the same time by Lewis [Lew70], yielding a result of $18540 \pm 90 \mathrm{eV}$. No statement about neutrino mass was made.

In 1972 the classic study of the tritium beta decay spectrum was published by Bergkvist [Ber72a, Ber72b]. Using a magnetic spectrometer with an extended source which was biased to inprove the luminosity, he 
was able to place an upper limit of $55 \mathrm{eV}$ on the neutrino mass. In addition, he outlined the basic experimental considerations necessary for a high-precision measurement of the mass. A review of the early tritium beta decay experiments (through Bergikvist) is given in Table 2-1 and Fig. 2,8.

Experimental Considerations in Tritium Beta Decay Experiments

Bergkvist pointed out that the presence of atomic electrons surrounding the decaying nucleus would change the observed spectrum in the vicinity of the endpoint. Failure to correct for these effects can lead to inaccurate estimates of the endpoint and neutrino mass. In most cases, the final states that will appear after the beta decay are not known because they depend on the chemical environment surrounding the tritium atom in the source. The effect of the final states on the spectrum can be seen by summing the spectra for each state:

$$
\frac{d N}{d E}=\sum_{i} A P_{i} P_{e} E\left(W_{i}-E\right)^{2}\left[1-\frac{m_{\nu}^{2}}{\left(W_{i}-E\right)^{2}}\right]^{1 / 2},
$$

where $P_{\mathfrak{I}}$ is the probability of ending up in state $i, A$ is a normalization factor, and $w_{i}$ is the endpoint of the $i$ th state, given by $W_{i}=E_{0}-m_{e} c^{2}-E_{i}$, where $E_{i}$ is the energy of state $i$. Assuming that $m_{\nu}=0$, and that the average endpoint $\langle W\rangle-\sum_{i} P_{i} W_{i}-E_{0}-\langle V\rangle$, then this expands into:

$$
\frac{d N}{d E}=A P_{e} E\left(E_{0}-E-\langle V\rangle\right)^{2}\left[1+\frac{\left\langle V^{2}\right\rangle-\langle V\rangle^{2}}{\left(E_{0}-E-\langle V\rangle\right)^{2}}\right],
$$


Table 2-1:

Summary of Early Tritium Results

\begin{tabular}{|c|c|c|c|}
\hline Investigator & Method & Endpoint (keV) & $\mathrm{m}_{\nu}(\mathrm{eV})$ \\
\hline Libby and Lee (1939) & Prop. Counter & $13 \pm 5$ & - \\
\hline Alvarez and Cornog (1940) & Ion Pairs & 18 & - \\
\hline o'Neal and Goldhaber (1940) & Range & $15 \pm 3$ & - \\
\hline Brown (1941) & Range & $9.5 \pm 2$ & - \\
\hline Neilsen (1941) & Cloud Chamber & $14.5 \pm 1$ & - \\
\hline Watts and Williams (1946) & Range & $11 \pm 2$ & - \\
\hline Byatt et al. (1948) & Cloud Chamber & $11 \pm 1$ & - \\
\hline Curran et al. (1948) & Prop. Counter & 16.9 & $<1700$ \\
\hline Hanna and Pontecorvo (1949) & Prop. Counter & 18.9 & - \\
\hline Jenks et al. $(1949,50)$ & Calorimeter & $18.6 \pm .2$ & - \\
\hline Byate et al. (1949) & Cloud Chamber & 17.0 & - \\
\hline Graves and Meyer (1949) & Bremsstrahlung & $18.0-.5$ & - \\
\hline Curran et al. (1949) & Prop. Counter & $18.3 \pm .3$ & $<1000$ \\
\hline Hamilton et al. (1952) & Elec. Spect. & $19.4 \pm .4$ & $<250$ \\
\hline Langer and Moffat (1952) & Mag. Spect. & $17.95 \pm .10$ & $<250$ \\
\hline Porter (1959) & Mag. Spect. & $18.61 \pm .1$ & - \\
\hline Salgo and Staub (1969) & Elec. Spect. & $18.7 \pm .1$ & $<200$ \\
\hline Daris and St.-Pierre (1969) & Mag. Spect. & $18.570 \pm .075$ & $<75$ \\
\hline Lewis (1970) & $S i(L i)$ & $18.540 \pm .090$ & - \\
\hline Bergkvist (1972) & Mag, Spect. & $18.610 \pm .016$ & $<55$ \\
\hline
\end{tabular}



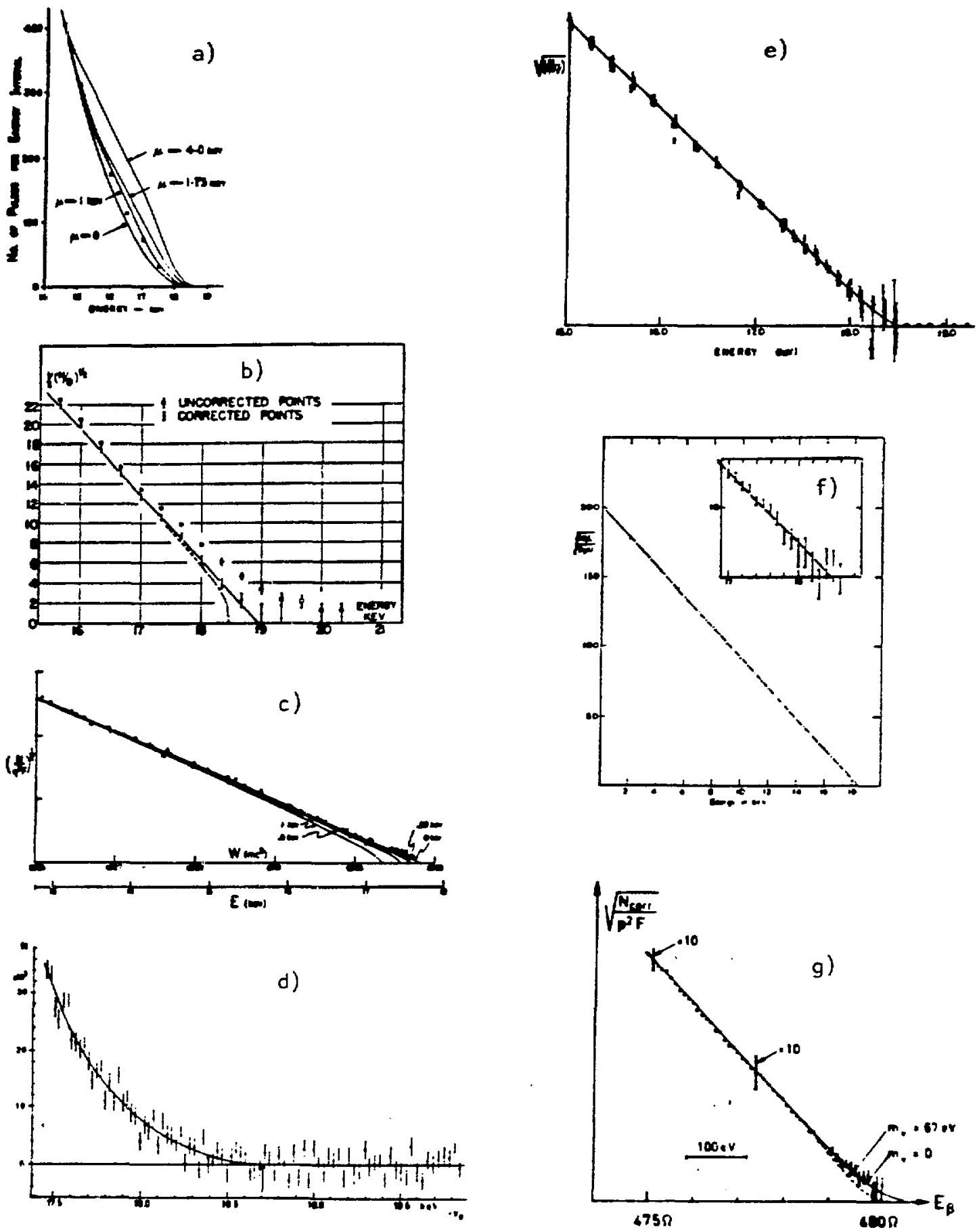

Fi8. 2.8. Summary of tritium beta spectrum results through 1972. a) Curran, Angus, and Cockroft [Cur49], b) Hanna and Pontecorvo [Haní;: c) Langer and Moffat [Lan52], d) Salgo and Staub [Sa169], e) Daris and St.-Pierre [Dar69], f) Lewis [Lew70], 8) Bergkvist [Ber72]. 
This looks like a spectrum with a shifted endpoint at $E_{0}-\langle V\rangle$ and a "neutrino mass" squared of $-2\left(\left\langle v^{2}\right\rangle-\langle v\rangle^{2}\right)$.

The atomic effects can be neglected in obtaining a lower limit for the neutrino mass because the curvature introduced into the spectrum by the final state effects is opposite to that introduced by the neutrino mass. Therefore, assuming that all the decays lead to a single state will yield a lower bound on the neutrino mass. Making this assumption inherently limits the experimental sensitivity to neutrino mass. Given an experiment with perfect instrumental resolution and atomic final states, it would be impossible to distinguish a neutrino mass of less than $20 \mathrm{eV}$ from zero if this "model-independent" lower bound were used. So, in order to attain a greater sensitivity to neutrino mass, information about the final states following beta decay is essential. In addition, it is impossible to set a "model-independent" upper bound on the neutrino mass. In order to set any upper bound at all, some assumption about the final state effects must be made. For the experiments before Bergkvist, the implicit assumption was that the width of the final state distribution was much less than the neutrino mass to which the experiments were sensitive. The width $\left\langle v^{2}\right\rangle^{1 / 2}$ of the atomic final state spectrum is about $40 \mathrm{eV}$, so any experiment setting an upper bound less than about twice that must account for the final state distribution.

The final state distributions for various materials which incorporate tritium can vary significantly, so it is in general not adequate to use the approximation of a free tritium atom to account for those states. Fig. 2.9 shows the final state distributions for free 

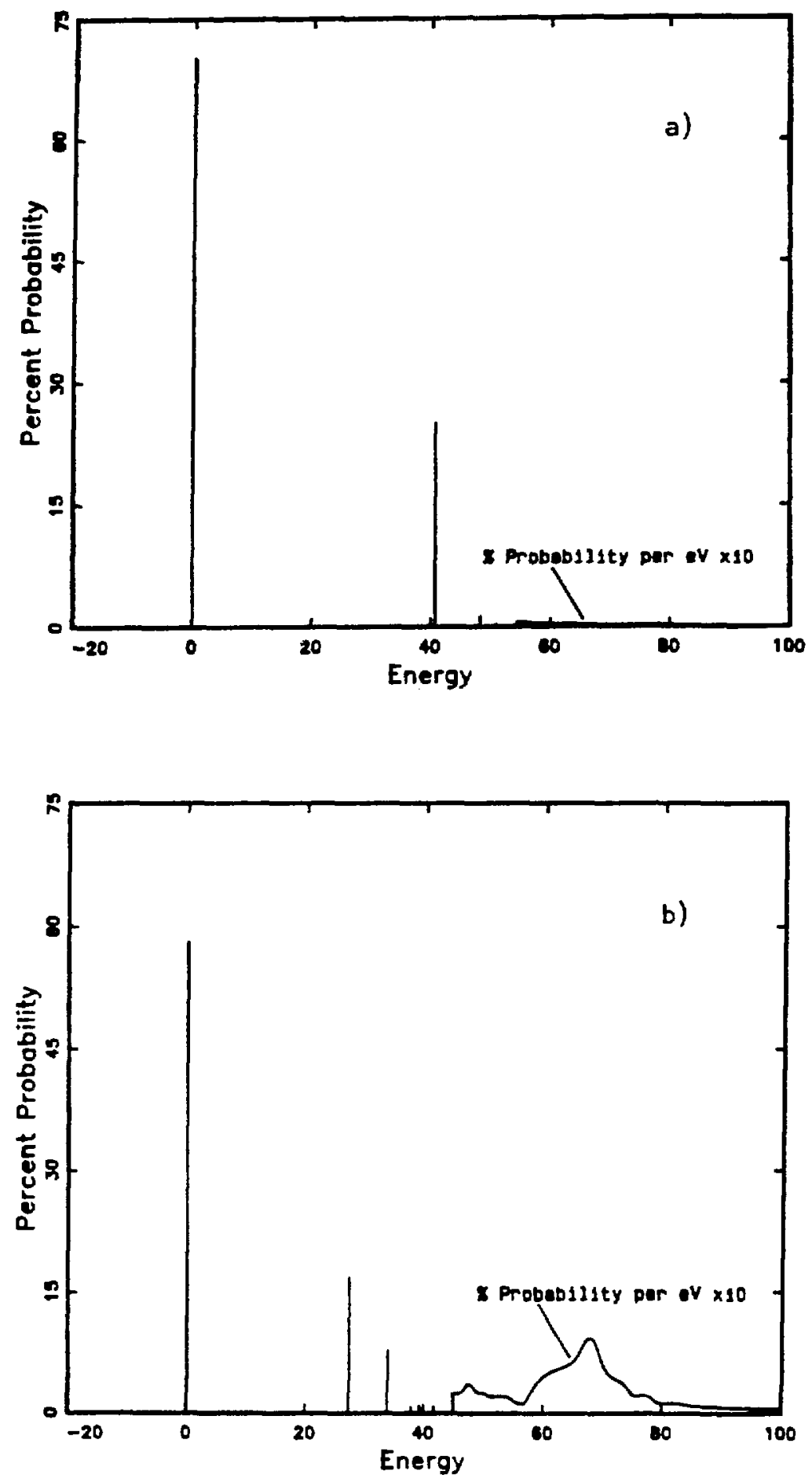

Fig. 2.9. Final state distributions for free tritium: a) atomic, and b) molecular. See Chapt. 5 for details of calculations. 
atomic and molecular tritium. The difference between even these two cases is very large. Any more complicated system will have a more complex distribution.

A second consideration that must be included in any sensitive measurement of the neutrino mass is the instrumental resolution. To first order, the effect of the resolution is the same as the effect of the final states, in that a resolution function tends to smear out the endpoint region of the spectrum. However, the resolution function does not shift the endpoint energy as long as the first moment $\langle R\rangle=0$, and it is always continuous, whereas the final state spectra may contain lines. This expands in a way similar to that of the final states, as Simpson has pointed out:

$$
\frac{d N}{d E}=A P_{e} E\left(E_{0}-E\right)^{2}\left[1+\frac{\left\langle R^{2}\right\rangle-\langle R\rangle^{2}}{\left(E_{0}-E\right)^{2}}\right] .
$$

In this case, $\langle R\rangle$ and $\left\langle R^{2}\right\rangle$ are the first and second moments of the resolution function, respectively. Since in most experiments the resolution function is defined to have $\langle R\rangle=0$, then the apparent "neutrino mass" squared is just $2\left\langle R^{2}\right\rangle$. For a resolution function, the characteristic width $\sigma$ is just $\left\langle R^{2}\right\rangle^{1 / 2}$, so an error in the resolution function width of $\Delta \sigma$ gives an apparent neutrino mass of $\left.2\left[\sigma \Delta \sigma+\Delta \sigma^{2} / 2\right)\right]^{1 / 2}$. Since $\Delta \sigma$ is usually much smaller than $\sigma$, this reduces to $2[\sigma \Delta \sigma]^{1 / 2}$. This implies that the resolution function must be known more accurately as it gets wider. Clearly, an experiment with as good a resolution as possible is desirable. In addition to the width of 
the resolution function, however, its shape must also be accurately known.

A third effect that can translate into neutrino mass is the background in the experiment. The last $100 \mathrm{eV}$ of the tritium beta spectrum contains only $2.3 \times 10^{-7}$ of the entire intensity, so it is imperative to keep the background minimal in order even to be able to see a neutrino mass signal. In addition, however, an inaccurately known background will appear as a neutrino mass. This can be seen by employing a somewhat different analysis. Doing an expansion assuming an error in the background rate of $\Delta B$ per eV gives:

$$
\frac{d N}{d E}=A p_{e} E\left(E_{0}-E\right)^{2}\left[1+\frac{\Delta B}{A p e E\left(E_{0}-E\right)^{2}}\right]
$$

Clearly the error in the neutrino mass depends on the ratio of the signal to the error in the background, but it "looks" in this equation like $-2 \triangle B / A p_{e} E$. Near the endpoint, the number of counts in a region from $E_{0}-K$ to $E_{0}$ for zero neutrino mass is given by:

$$
\mathrm{N}(\mathrm{K}) \approx \mathrm{AP}_{\mathrm{e}} \mathrm{E} \mathrm{K}^{3} / 3
$$

and the number of erroneous background counts would be given by:

$$
N_{B}(K)=(\Delta B) K
$$

So the apparent neutrino mass caused by the error in the background is given by:

$$
m_{\nu}^{2} \approx \frac{-2 K^{2} N_{B}(K)}{3(K)} \approx \frac{-2 \Delta B}{A P_{e} E} .
$$


It should be noted that this expression does not depend on $K$ as long as $\mathrm{K}$ is small compared to $\mathrm{E}_{0}$ and $\mathrm{K}>0$. A convenient way of interpreting this expression is to choose a value of $K$ for which $N_{B}(K)-N(K)$, in which case $m_{\nu}^{2}=-2 / 3 \mathrm{~K}^{2}$.

All the expressions so far have assumed a constant background as a function of energy. The problems associated with errors in the background become much more difficult to deal with when the background varies with emitted electron energy.

Other significant factors to consider in the development of a neutrino mass measurement using tritium are the efficiency of the detection apparatus as a function of energy and the energy loss that can occur in the source. A miscalibration of the efficiency of detection may lead to an incorrect endpoint extrapolation, which will in turn lead to an apparent neutrino mass. In addition, the curvature which is introduced into the spectrum by the efficiency function could very well mimic a neutrino mass. The sensitivity of an experiment to errors in the determination of the efficiency function depends on the resolution, background, and statistical accuracy as well as the magnitude of the variations in the efficiency function. An incorrect determination of energy loss in the source will affect the neutrino mass in exactly the same way as an incorrect resolution function, and can, since the mean energy loss is not zerc, lead to an incorrect determination of the endpoint energy. 
Measurements of the Q-value in Tritium Beta Decay

A measurement of particular interest to the determination of neutrino mass from tritium is that of the mass difference between tritium and ${ }^{3}$ He. The measurement of the beta spectrum is one way to determine this value, but since these measurements are more often oriented toward finding the neutrino mass, an independent measurement of the mass difference can be used to either check the results from the spectral. shape or to improve the accuracy of those results, if the energy calibration of the spectrometer is well enough known and the error in the mass difference is less than the statistical error of the endpoint from the spectrum. Table 2-2 shows the measurements which have been made of this mass difference using methods other than the measurement of the beta spectrum. The best value is probably that of Lipmaa et al. [Lip85], who obtained a value of $18599 \pm 3 \mathrm{eV}$ by comparing the ion cyclotron resonance frequencies of ${ }^{3} \mathrm{H}^{+}$and ${ }^{3} \mathrm{He}^{+}$.

Methods of Measuring the Tritium Beta Spectrum

There are several experimental methods that are currently employed to measure the tritium beta spectrum. Each of them has its strong and weak points in view of the considerations raised above.

One method is the use of a magnetic spectrometer, which analyzes the energy of the betas by measuring their curvature in a magnetic field. This method is relatively insensitive to background, and spectrometers can be designed to measure a fairly wide range of energies simultaneously. However, in a magnetic spectrometer the luminosity and 
Table 2-2:

Summary of Q-Value Measurements

\begin{tabular}{lll}
\multicolumn{1}{c}{ Investigator } & Method & Q-Value \\
\hline Bergkvist (1972) & Beta Spectrum & $18645 \pm 16$ \\
Piel (1973) & Beta Spectrum & $18619 \pm 40$ \\
Smith (1975) & RF Spectr. Doublet & $18595 \pm 7$ \\
Smith (1981) & RF Spectr. Doublet & $18579 \pm 8$ \\
Simpson (1981) & Si(Li) Implantation & $18567 \pm 20$ \\
Dixon (1982) & Si(Li) Implantation & $18594 \pm 25$ \\
Derbin (1983) & Si(Li) Implantation & $18562 \pm 20$ \\
Lippmaa (1984) & ICR Doublet & $18599 \pm 3$ \\
Nikolaev (1984) & ICR Doublet & $18584 \pm 4$ \\
Simpson (1985) & Si(Li) Implantation & $18580 \pm 7$ \\
Lippmaa (1985) & ICR Doublet & $18599 \pm 2$
\end{tabular}


resolution tend to be inversely related $\left(\Delta \mathrm{p} / \mathrm{p}-\mathrm{L}^{-2}\right)$, so that in order to obtain a reasonable count rate a relatively poor experimental resolution must be accepted.

Electrostatic deceleration spectrometers have by far the best resolution of any method. In this type of experiment the betas are decelerated by a grid at some potential $V$, so that only those electrons with kinetic energy greater than $\mathrm{V}$ can pass through the grid plane. The resolution of these spectrometers is mainly determined by the uniformity of the voltage across the grid plane. Electrostatic spectrometers can be built with very large acceptances at a minimal cost in resolution. However, they are extremely sensitive to background, since the electrons of interest have zero energy at the grid plane, and any other process that creates low energy electrons, such as photoemission, corona discharge, or residual tritium gas, will produce a background which cannot be distinguished from the signal.

Hybrids between magnetic and electrostatic spectrometers can eliminate some of the disadvantages of each. Varying the potential across the surface of a source has been used to improve the acceptance and resolution of magnetic spectrometers [Ber72, Lyu83, Fri86]. Deceleration of the betas to a lower energy (but not to zero energy, as in an electrostatic spectrometer) can improve the resolution of a magnetic spectrometer $[$ Pet86].

Solid state detectors can also be used to measure the spectrum, and have several advantages. Tritium can be implanted into the detector itself, so that all the energy from the decay except that which goes to the neutrino is detected. - This eliminates the need for atomic final 
state corrections. There is no efficiency variation, and no energy loss in the source. However, the total count rate must be kept low enough to keep the deadtime and pileup within reasonable limits, which means that the count rate of electrons with energies near the endpoint is extremely small. In addition, the resolution of solid state detectors is very poor compared to that of either electrostatic or magnetic spectrometers.

\section{The ITEP Results}

Much of the current interest in the measurement of the electron antineutrino mass stems from measurements done by a group at ITEP, in Moscow. In 1975 Tret'yakov reported on the construction of an iron-free toroidal field beta spectrometer [Tre75] that had a very good resolution and high luminosity. This spectrometer was used immediately in measurements of the beta spectrum of tritium, and an upper limit on the neutrino mass of $33 \mathrm{eV}$ was published [Tre76]. In 1980, however, the same group published a paper which claimed evidence for a non-zero neutrino mass, of $14 \leq m_{\nu} \leq 46 \mathrm{eV}$ [Lyu80a], with a best-fit value of $34 \mathrm{eV}$. The lower limit was obtained by the use of the "modelindependent" assumption about the atomic final states. The experimental procedure was amplified in later papers [Lyu80b, Lyu81], and provoked a strong discussion. A further paper was released in 1983 [Lyu83] in which more data was presented, still pointing to a nonzero neutrino mass. In the 1983 paper, however, the fit of a theoretical curve with a single neutrino mass to the data was so poor for the best fit value of $33 \mathrm{eV}$ that two neutrino masses were invoked to explain the discrepancies. The two masses and mixing weights were given as: 


$$
\begin{array}{lll}
0 \leq \mathrm{m}_{1} \leq 30 \mathrm{eV} & \text { with } & .75 \leq \mathrm{w}_{1} \leq .95 \\
80 \leq \mathrm{m}_{2} \leq 120 \mathrm{eV} \text { with } & .05 \leq \mathrm{w}_{2} \leq .25
\end{array}
$$

Another paper was published in 1985 [Bor85], in which the positive neutrino mass result was upheld, with a "valine" neutrino mass value of $34.8 \pm 1.9 \mathrm{eV}$ and a "model-independent" lower limit of $13.8+2.5 /-3.5 \mathrm{eV}$. A re-analysis of this data was presented at the Moriond conference in Jan. 1986 [Lyu86]. It consisted mainly of a more careful analysis of the shape of the experimental resolution function, and a new set of limits was announced. The "model-independent" lower limit has been eliminated because it generates an endpoint which is incompatible with the ion cyclotron resonance measurement of the $\mathrm{T}-{ }^{3} \mathrm{He}^{+}$mass difference made by Lipmaa et al. [Lip85]. Instead, the limits set were defined as those for which the fit is in agreement with this measurement. This yields a mass range of $17<\mathrm{m}_{i}<40 \mathrm{eV}$ and a best-fit value of $30.1 \pm 2 \mathrm{eV}$. The residuals plot for this data is shown in Fig. 2.10, and the total resolution function used in the analysis in Fig. 2.11.

These measurements have been subjected to close scrutiny since their publication. Simpson [Sim82] wrote to Lyubimov expressing concern that the intrinsic width of the $M$ lines in the ${ }^{169} \mathrm{Yb}$ (the lines are those of $\mathrm{Tm}$, the daughter atom) conversion source that was used to determine the resolution of the Tret'yakov spectrometer had been neglected in the determination of the resolution. This, of course, would lead to an overestimation of the width of the resolution function and therefore to an overestimation of the neutrino mass. Simpson 


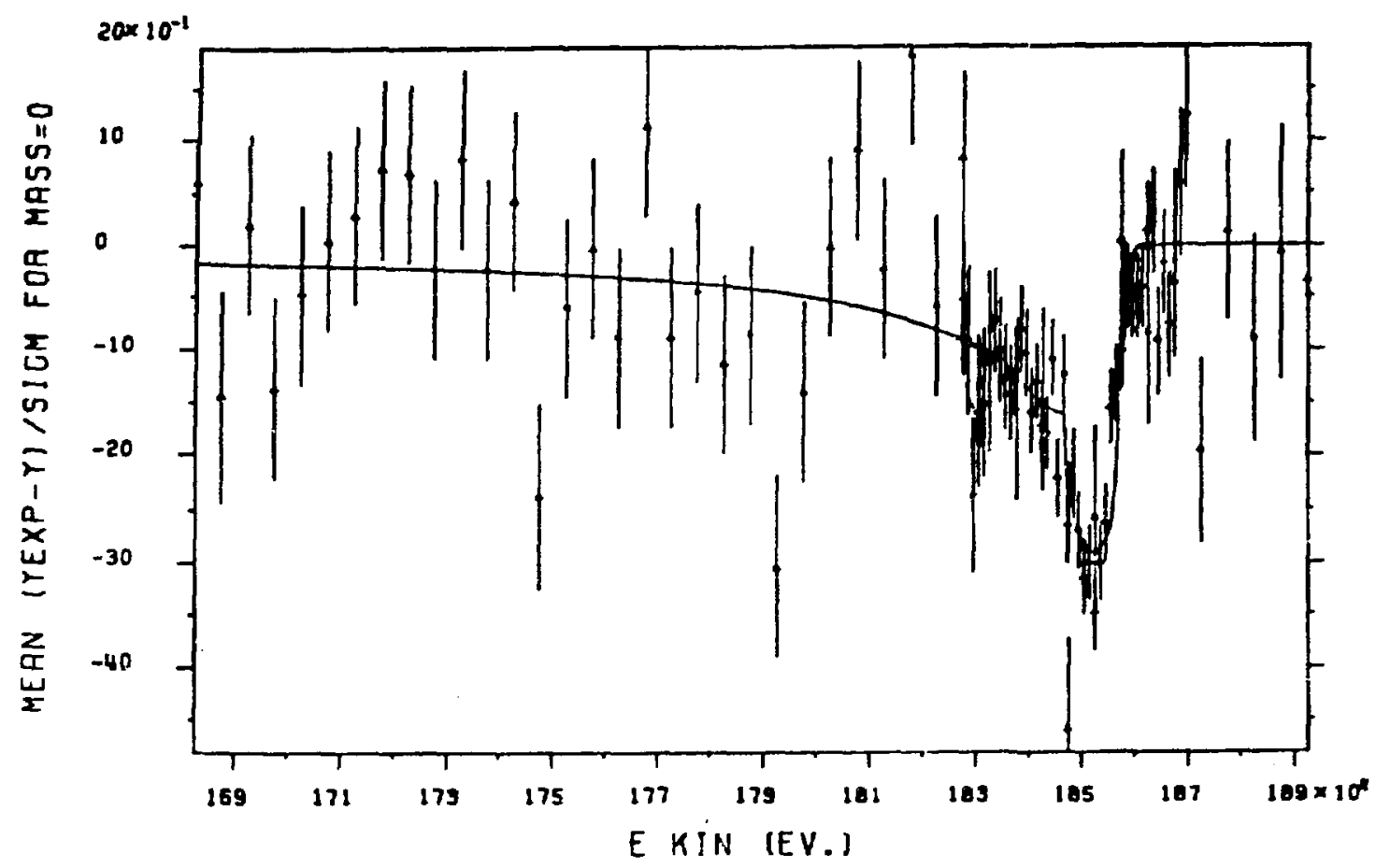

F18. 2.10. ITEP 1986 residuals [Lyu86]. It is not known whether the line for zero neutrino mass has any other parameters fitted.

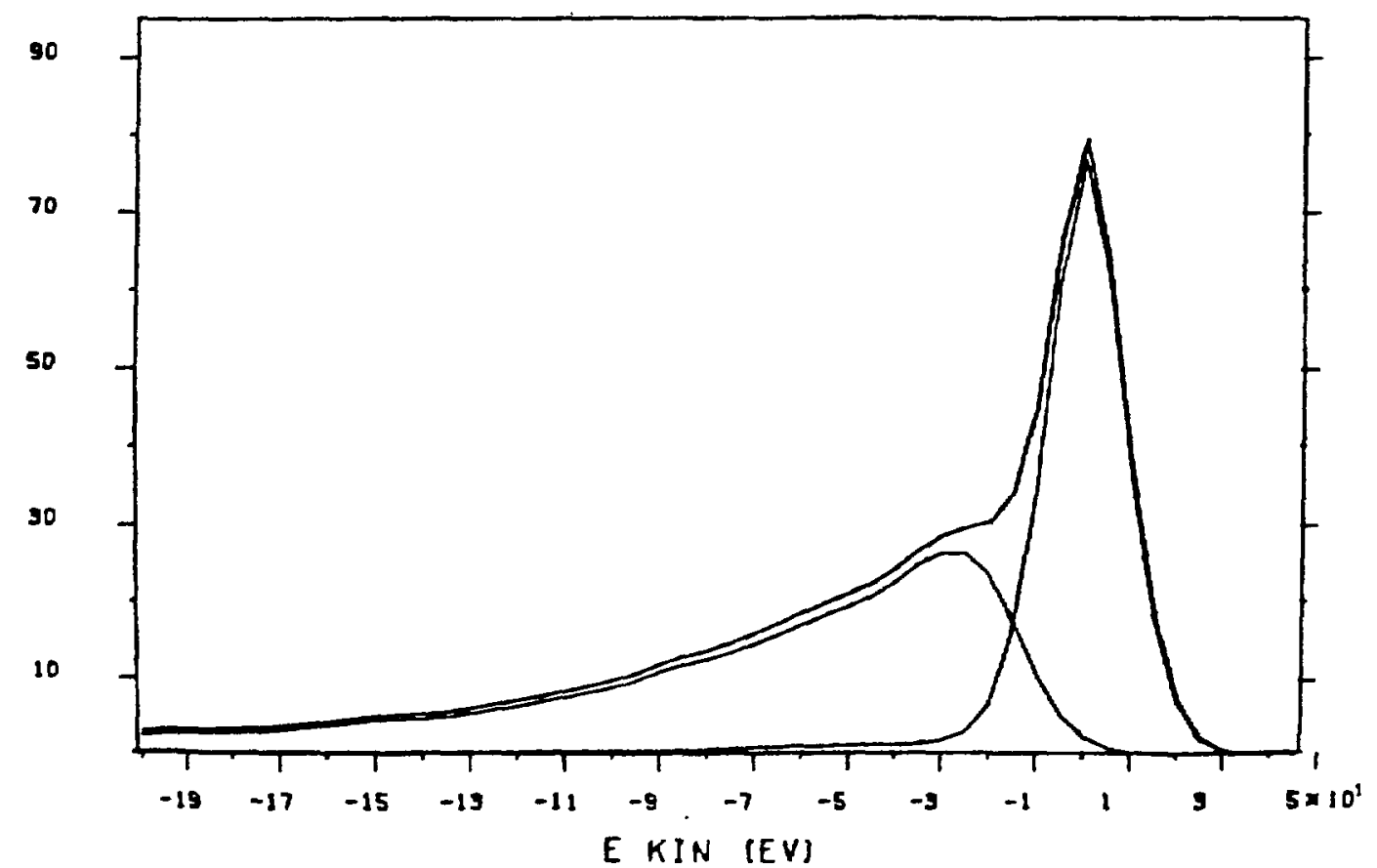

F18. 2.11. The ITEP 1986 total resolution function, including the resolution width of the spectrometer and the ionization loss spectrum (IIS). 
claimed that this problem effectively destroyed the "model-independent" lower limit, but did not entirely account for the observed central value. Bergkvist also wrote to Lyubimov, and commented on the same points [Ber82]. He did not, however, believe that the neglect of the width of the lines was sufficient to explain away the positive mass result.

Lyubimov replied to Simpson [Lyu82] that these concerns had been more recently addressed, that the widths of the lines had been determined, and that the inclusion of the width of the lines reduced the neutrino mass from 34 to $28 \mathrm{eV}$.

Bennett et al. measured the $T_{m}$ line widths after the publication of the 1983 data [Ben85], and found that the widths agreed with the ITEP measurements mentioned by Lyubimov in his letter to Simpson. However, their analysis indicated that the new "best fit" neutrino mass would fall at $24 \mathrm{eV}$. In addition, they analyzed the 1980 and 1983 ITEP data and concluded that in both cases there was not evidence for a nonzero neutrino mass. A possible explanation of the two-neutrino effect seen in the 1983 data was presented, this being that there was a shift in the endpoint energy between the data taken near the endpoint and the data taken far away (see Fig. 2.12).

Further comments on the ITEP-83 and ITEP-85 data have been published by Bergkvist [Ber85a, Ber85b]. He has argued that the shape of the long tails in the resolution resolution function in both ITEP papers is due mainly to shake-off in the $\operatorname{Im}$ atom, not to solid state effects, as is claimed by the ITEP group. If this is indeed the case, then the actual width of the resolution function of the ITEP 


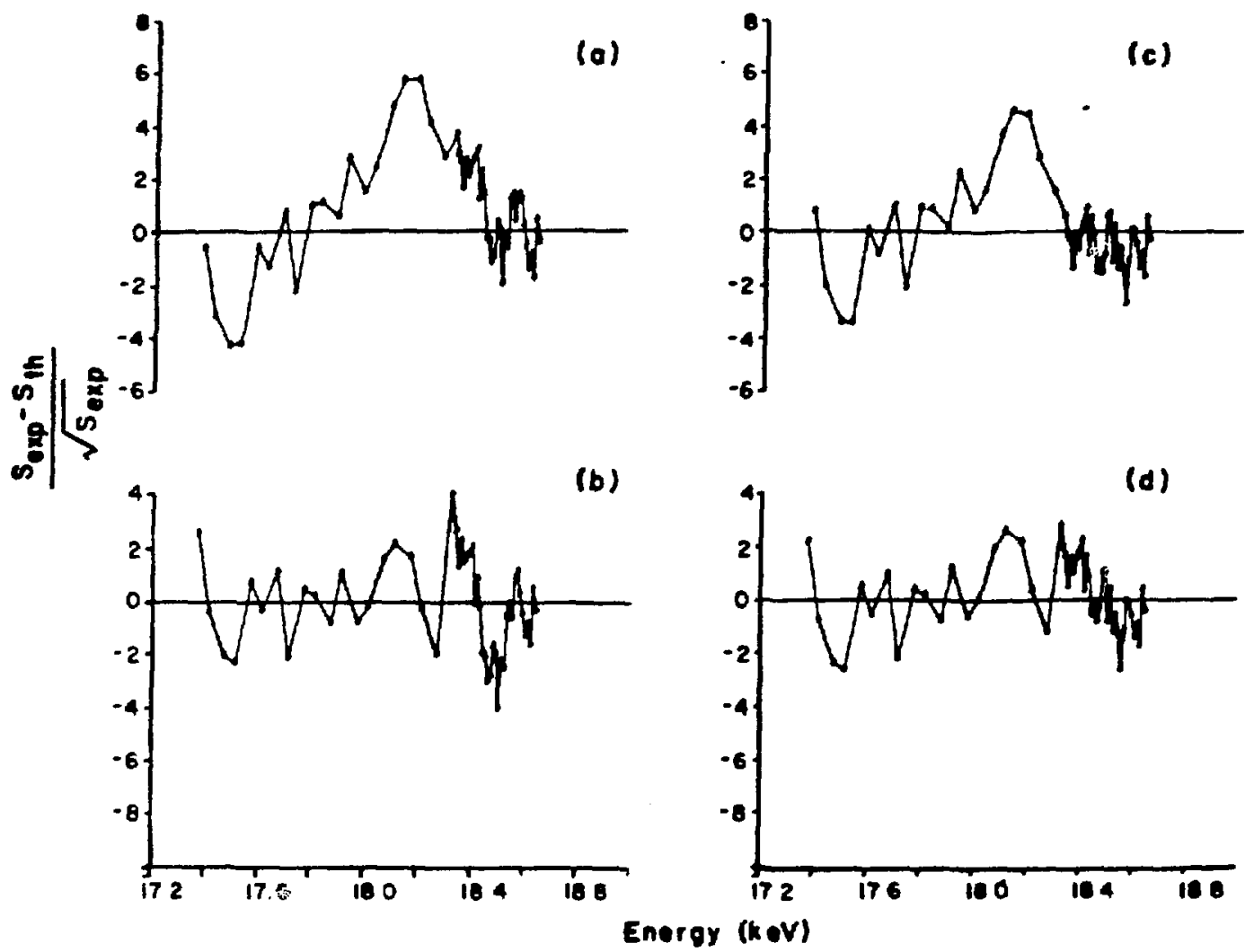

Fig. 2.12. The ITEP 1983 residuals [Lyu83] analyzed by Bennett et al. [Ben85]. a) residuals for $m_{\nu}=0, E_{0}=18568 \mathrm{eV}$. b) residuals for $m_{\nu}=0, \quad E_{0}=18571 \mathrm{eV}$, with a $-10 \mathrm{eV}$ shift for data points with $E<18.2 \mathrm{keV}$. c) residuals for 2 neutrino masses: $70 \% \mathrm{~m}_{\nu}=0$ and $30 \%$ $m_{\nu}=80 \mathrm{eV}$, with $E_{0}=18575 \mathrm{eV}$. d) same as c) but with data for $\mathrm{E}<18.2 \mathrm{keV}$ shifted by $-7 \mathrm{eV}$. 
spectrometer is much less than that which was used in the analysis, and the case for the neutrino mass is very much weaker. Indeed, using an argument based on the mean square energy spreads of various components of the spectrum, which he measured with the same spectrometer that was used for his original tritium measurements, Bergkvist states that the ITEP claims of a nonzero mass are false. However, an analysis using the mean square energy spread is not valid for resolution functions with long tails, so that the results are in doubt until a detailed calculation which takes the shapes into account is done.

The largest barrier to acceptance of the ITEP result has been an incomplete understanding of what was actually done. Very little communication between the group and Westerners has taken place since the 1980 result. Therefore, the claims have been difficult at best to interpret. It is, however, of paramount importance that the question about the rest mass of the neutrino be answered as unambiguously as possible by new experiments.

\section{New Results from Tritium Beta Decay}

The only new result from tritium beta decay that has been announced is that of Petersen et al. [Pet86, Fri86]. Their experiment uses a solid source of tritium implanted into amorphous carbon and a toroidal magnetic spectrometer into which the electrons are decelerated in order to improve the resolution. The data is of high quality, with a very high statistical accuracy. The analysis of the data included a fit to the final states as well as to the other parameters. Data presented at the Moriond meeting in January 1986 [Pet86] is shown in Fig. 2.13. 
The response function used in the analysis of this data is shown in Fig. 2.14. It consists of an optical resolution function determined by a Monte Carlo simulation added to an energy loss spectrum determined from calculations of energy loss in the carbon substrate folded with the predicted depth profile of the tritium in the carbon. An upper limit of $21.7 \mathrm{eV}$, including ail systematic errors added coherently, has been derived from this data. Clearly, this data does not support the ITEP central value, although the extreme limits are marginally in agreement.

\section{Other Experiments to Determine the Neutrino Mass}

The measurement of the shape of the beta spectrum of tritium is not the only method that has been proposed to measure the electron neutrino mass. Other methods can be used to measure the mass, but each has disadvantages when compared to tritium beta decay.

In 1980 de Rujula and Bennett proposed that the neutrino mass could be measured by observing the shape of the internal bremsstrahlung and $x$-ray spectrum following a low $Q$-value electron capture $[R u j 81$, Ben80]. The neutrino mass effects that appear in the phase space of tritium beta decay will manifest themselves in a similar way in electron capture; however, in this case the mass reduces the phase space available to the bremsstrahlung photons. However, the bremsstrahlung experiments rely on resonant enhancement of the internal bremsstrahlung amplitude from $p$-wave captures at and below the characteristic $x$-ray energies, and on finding a Q-value sufficiently low that neutrino mass effects are substantial. 


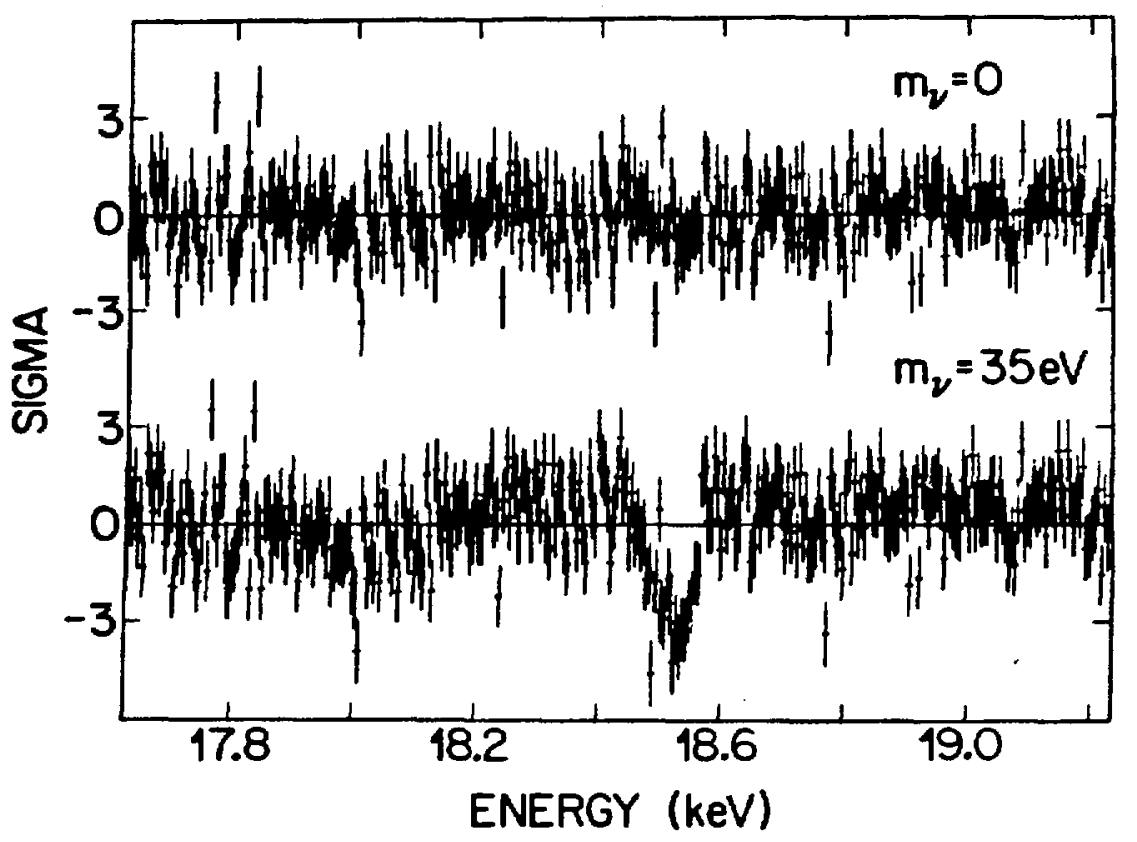

Fig. 2.13. Zurich 1986 [Kun86] residuals with neutrino mass $=0$ and 35 eV. All free parameters were fitted in both plots.

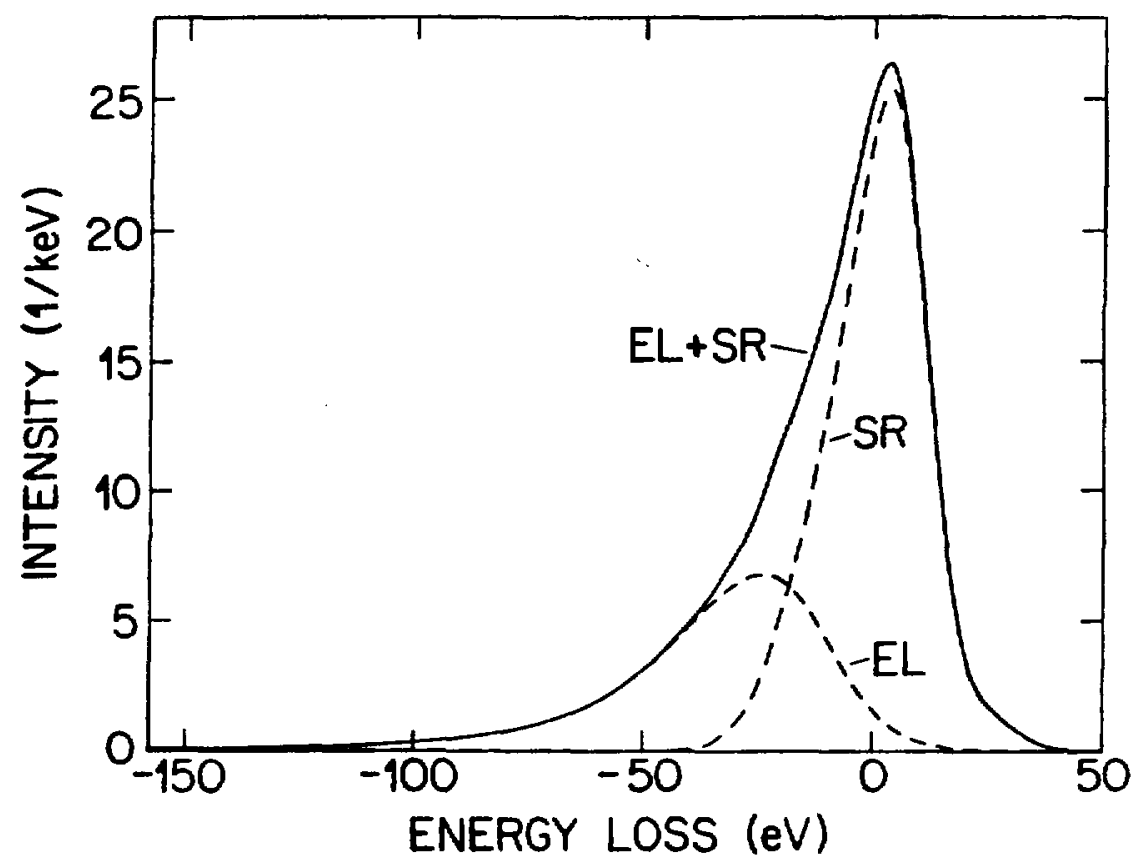

Fig. 2.14. The Zurich 1986 total resolution function. 
When it was first proposed, the IBEC (Internal Bremsstrahlung Electron Capture) method of measuring neutrino mass generated a great deal of excitement. Like tritium beta decay, this method does not require any special properties of the neutrino other than a mass in order to see a result, and it has the advantage of looking directly at the electron neutrino rest mass, rather than the mass of the electron antineutrino, as is the case in tritium beta decay experiments. Two promising nuclei, ${ }^{163} \mathrm{Ho}$ and ${ }^{193} \mathrm{Pt}$, were mentioned by de Rujula.

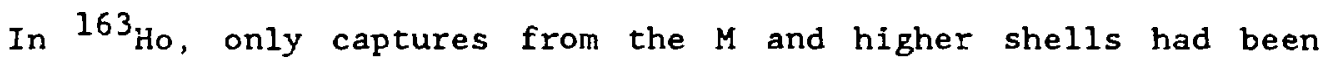
observed, and the Q-value was at the time taken to be between 2 and $10 \mathrm{keV}$. If the Q-value were to fall near an $\mathrm{x}$-ray pole, the possibilities for seeing a neutrino mass would be quite promising. In ${ }^{193} \mathrm{Pt}$, the Q-value was measured to be around $60 \mathrm{keV}$, which would make it just barely possible to observe a neutrino mass of the order of tens of $\mathrm{eV}$ (de Rujula estimated 17 events per year in the region of interest).

In 1983, Raghavan reported on an ultralow energy K-capture in ${ }^{158} \mathrm{~Tb}$, which could be used in a way similar to the IBEC method for measuring the neutrino mass [Rag83]. He reported the observation of $K$ $x$-rays in coincidence with gammas from the daughter nucleus. From the measured subshell ratios, the available energy for K-capture would be just $156 \mathrm{eV}$, making it exrremely sensitive to the neutrino mass.

The potentially very exciting case of ${ }^{158} \mathrm{~Tb}$ was ruled out by Altzitzoglou et al. [Alt85], LoSecco et al. [Los85], and Brudanin et al. [Bru85]. Altzitzoglou et al. measured the Q-value of the transition and found it too low to allow $K$-capture. The other groups performed more precise coincidence measurements, which indicated that K-capture should 
not be energetically allowed, and did not observe capture from the $k$ shell. It appears that the $\mathrm{K}$-rays reported by Raghavan were random coincidences.

Measurements using $163_{\text {Ho }}$ were made by Bennett et al. [Ben81, Ben84]. The 1981 results were a measurement of the $x$-ray spectrum, the idea being that the neutrino mass could be determined from the relative capture rates from different orbitals. The sensitivity of this method to neutrino mass was shown to be a very strong function of the Q-value and the neutrino mass. The 1981 measurements gave a lower bound of $2.1 \mathrm{keV}$ on the Q-value, but a neutrino mass could not yet be obtained. The measurements were completed in 1984, and an upper limit on the neutrino mass of $225 \mathrm{eV}$ was set. In addition, however, this group determined that the IBEC spectrum of ${ }^{163}$ Ho could never be as sensitive to the neutrino mass as the spectrum of tritium since the interference of atomic effects from higher lying states is too significant and is not calculable.

The ISOLDE group at CERN also measured the spectrum of $163^{\mathrm{Ho}}$ [And82]. Their measurement of the Q-value was consistent with the measurement by Bennett et al., and they measured the partial half-life of $M$ capture to be $(4.0 \pm 1.2) \times 10^{4}$ years. From this they were able to infer an upper limit on the neutrino mass of $1.3 \mathrm{keV}$.

Another measurement of the ${ }^{163}$ Ho spectrum was made by Yasumi et al. [Yas83], who also determined the partial half-life of $M$ capture. Their measured value was $(4.5 \pm 1.5) \times 10^{4}$ years. Using this and the upper limit on the Q-value measured by Andersen et aI. [And82], an upper limit on the electron neutrino mass of $1.25 \mathrm{keV}$ was obtained. 
The CERN group also measured the IBEC spectrum of ${ }^{193} \mathrm{Pt}$ [Jon83]. The data in this case made it possible to obtain a better limit on the neutrino mass than in the case of ${ }^{163}$ Ho. Using a method of analysis strikingly similar to that used in tritium experiments, a limit of $m_{\nu}<500$ eV was obtained.

Another method that has been proposed for the determination of the neutrino mass is the detection of neutrino oscillations. The idea that neutrinos might oscillate is not new, but it is not as general a test for neutrino mass as tritium beta decay or IBEC, because it requires lepton number violation as well as massive neutrinos. Basically, neutrino oscillation experiments investigate the reactions:

$$
\nu_{1} \rightarrow \nu_{2}, \nu_{1}+\bar{\nu}_{1} \text {, or } \nu_{1} \rightarrow \text { anything, }
$$

where $\nu_{1}$ and $\nu_{2}$ are any two neutrino flavors. The experiments either look for the disappearance of one kind of neutrino or the appearance of another. The parameters to which they are sensitive are $\Delta m^{2}$ and $\sin ^{2} 2 \theta$, where $\Delta m^{2}=\left|m_{1}^{2}-m_{2}^{2}\right|$, and $\theta$ is the mixing angle between the flavors.

An enormous number of neutrino oscillation experiments have been undertaken in the last several years. For the sake of brevity, the majority of these experiments will be skipped. The current world best limits on neutrino oscillations are shown in Fig. 2.15. A positive result for the oscillation $\nu_{e} \rightarrow$ anything was obtained by the Bugey collaboration in 1984 [Koa84, Cav84]. The data, shown in Fig. 2.16, clearly indicate a deficit of neutrinos detected at $18.3 \mathrm{~m}$ from the reactor core. Taken with the other data, this would indicate values for $\Delta \mathrm{m}^{2}$ of about $.2 \mathrm{eV}^{2}$ and $\sin ^{2} 2 \theta$ of about .2 as well. This evidence is 


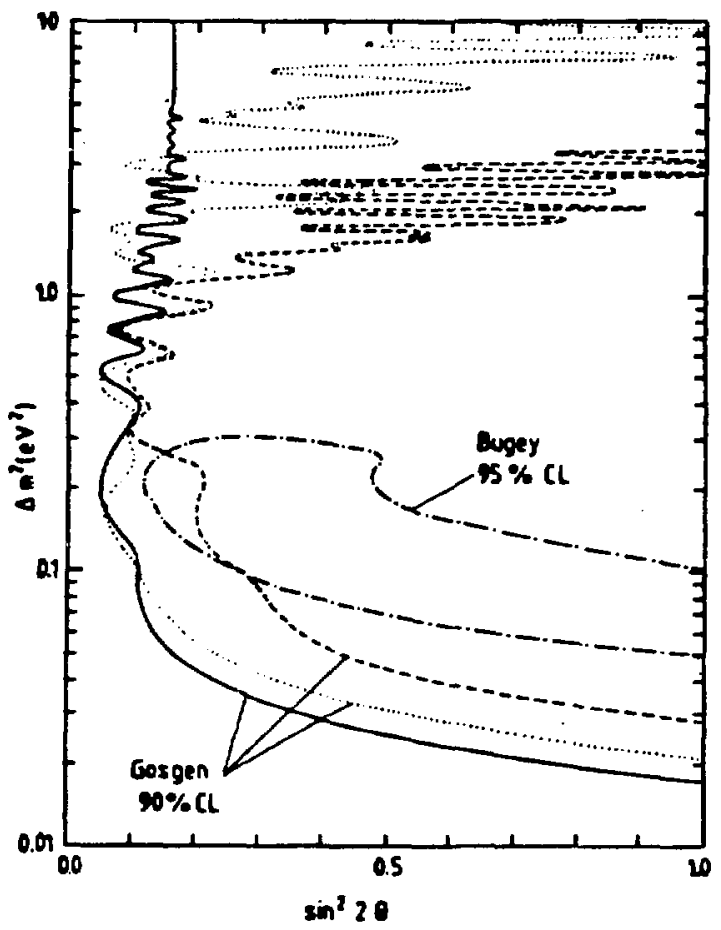

Fig. 2.15. Neutrino oscillation limits from the Bugey and Goesgen experiments [Koa84 and Vui84]. The Bugey result is contained to the right of the line, while the Goesgen result excludes the region to the right of its line.
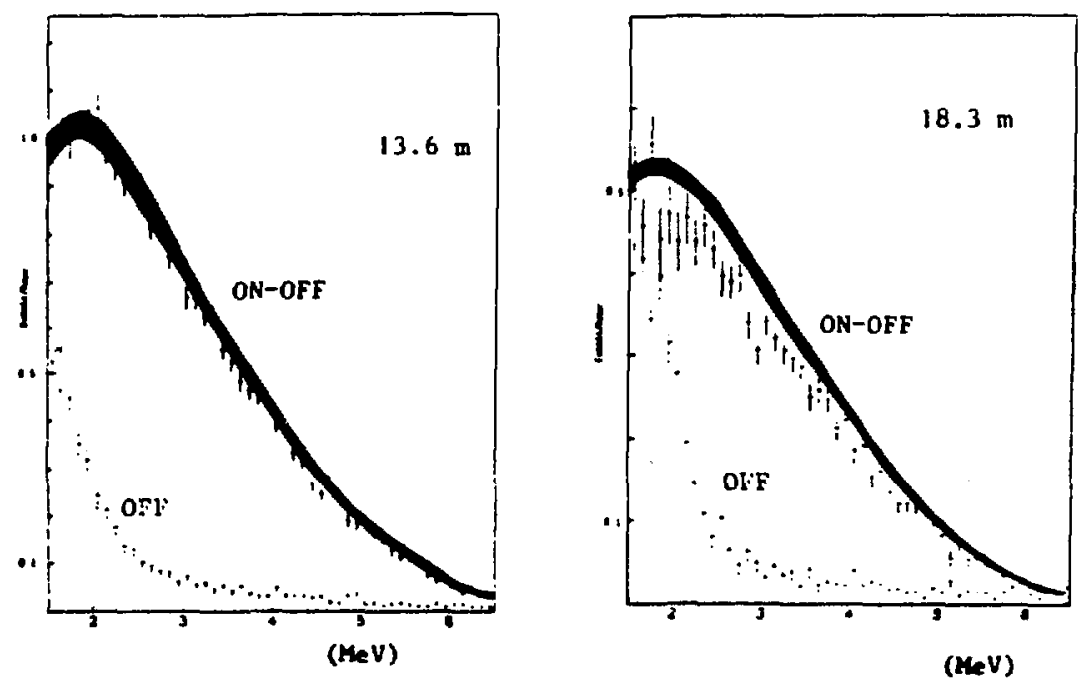

Fig. 2.16. The Bugey data [Koa84]. Positron energy spectra are shown. The dark bands are Monte Carlo calculations delimited by point-to-point error, assuming no oscillations. 
inconsistent with that obtained by another group doing an extremely similar experiment [Vui84, Boe84] in which no evidence for oscillations has been observed.

The only other significant type of experiment which is being used to search for a neutrino mass is the investigation of neutrinoless double beta decay. This process can only occur if the neutrino is a Majorana particle (that is, its own antiparticle) and has mass [Tak84]. It can be distinguished from the two-neutrino decay by the energy spectrum of the beta particles. In $2 \nu \beta \beta$ decay, the beta spectrum is a continuum, just as it is in the more familiar case of single beta decay. In $O_{\nu} \beta \beta$ decay, however, the sum of the beta energies is constant. Therefore, many double beta decay experiments search for these monoenergetic signals. The most popular isotope to use in this search has been ${ }^{76} \mathrm{Ge}$, which occurs in natural germanium and therefore is present in any germanium particle detector. Other isotopes which have been studied are ${ }^{82} \mathrm{Se},{ }^{150} \mathrm{Nd},{ }^{136} \mathrm{Xe}$, and ${ }^{48} \mathrm{Ca}$. Given assumptions about decay probabilities, matrix elements, and neutrino types, double beta decay experiments can set very stringent upper limits on the neutrino mass. The best limits from ${ }^{76} \mathrm{Ge}$ and ${ }^{82} \mathrm{Se}$ are both under $10 \mathrm{eV}$ [Moe80, Be183, For83], although there is some uncertainty about the actual limits due to conflicting experimental results [Hax83]. The most stringent limit set by geochemical data (measuring the half-lives of ${ }^{130} \mathrm{Te},{ }^{128} \mathrm{Te}$, and ${ }^{82} \mathrm{Se}$ ) is an upper limit on the neutrino mass of about $6 \mathrm{eV}$, obtained from the ratio of the decay rates for ${ }^{128} \mathrm{Te}$ and ${ }^{130} \mathrm{Te}$ [Kir83]. It is important to note in this case that the observed $2 \nu$ 
decays for both ${ }^{130} \mathrm{Te}$ and ${ }^{128} \mathrm{Te}$ have half-lives more than an order of magnitude longer than is predicted by theory.

Both neutrino oscillation experiments and neutrinoless double beta decay experiments promise an extremely high sensitivity to neutrino mass; however, since lepton number violation is required for both, these measurements cannot be directly compared to tritium beta decay or to IBEC experiments. A null result in one of these experiments does not necessarily imply that the neutrino has no mass; rather, it means that lepton number violation has not been observed. 
Chapter 3:

The Current Experiment

The Los Alamos tritium beta decay experiment has been designed to give as unambiguous a beta spectrum as possible, so that the neutrino mass and endpoint can be determined with a minimum of systematic error. Al1 high-resolution tritium beta decay experiments which have been performed to date have used solid sources with tritium in them, and each of these experiments has had difficulty with interpretation of the data due to effects in the source.

\section{Difficulties with Solid Sources}

There are several problems encountered involving the use of solid sources in tritium beta decay experiments. The most obvious is, of course, the question of the final state effects (see Chapt. 2). Those effects which can be calculated accurately have only been done for the free tritium atom or molecule. Any solid has some binding energy that changes the wavefunctions of the atomic electrons and thereby the final state spectrum. This effect is probably most significant in the case of a tritiated compound such as the valine the ITEP group used, but may ronetheless be important in implanted or frozen sources. The final state energies may be further changed by the dielectric constant of the solid medium, which changes the effective electric field seen by the atcmic electrons and thereby the energies of the eigenstates. If the material of the solid source is not a conductor, the source can charge up, changing the original potential of the emitted electrons. 
A second important effect that occurs in solid sources can be seen by examination of the 1985 ITEP data [Bor85]. While the FWHM of the optical resolution function is fairly narrow (about $25 \mathrm{eV}$ ), there is a long tail due to the "ionization loss spectrum" which extends for more than $250 \mathrm{eV}$ (see Fig. 3.1). This "ILS" is probably dominated by the plasmon excitation spectrum, which is a feature of thin layers of material. Clearly, the largest component of this resolution function is due to solid state effects, and could not be eliminated even with perfect optical resolution. Bergkvist [Ber85a, Ber85b] has asserted that this low energy tail is at least partly a result of the shake-off spectrum of the calibration source, and should not be actually included in the resolution function. Either way, the true resolution function is extremely difficult to determine for a solid source.

Another problem that affects all sources, but is especially severe in the case of solid sources, is the effect of elastic scattering on the solid angle. Any source which has a "preferred direction" (that is, a direction particles can go in which the scattering probability is less than that of other directions) will tend to emit more particles in that direction. The magnitude of this effect depends on the cross section For elastic scattering, which is a function of energy. Therefore, the accepted solid angle for an apparatus using this type of source will vary with energy. A solid source has a preferred direction, which is away from the source. Since any beta decay experiment using a solid source will make use of these electrons, this effect is unavoidable, and since the energy of the electrons is very low in the case of tritium, it could potentially be significant. 


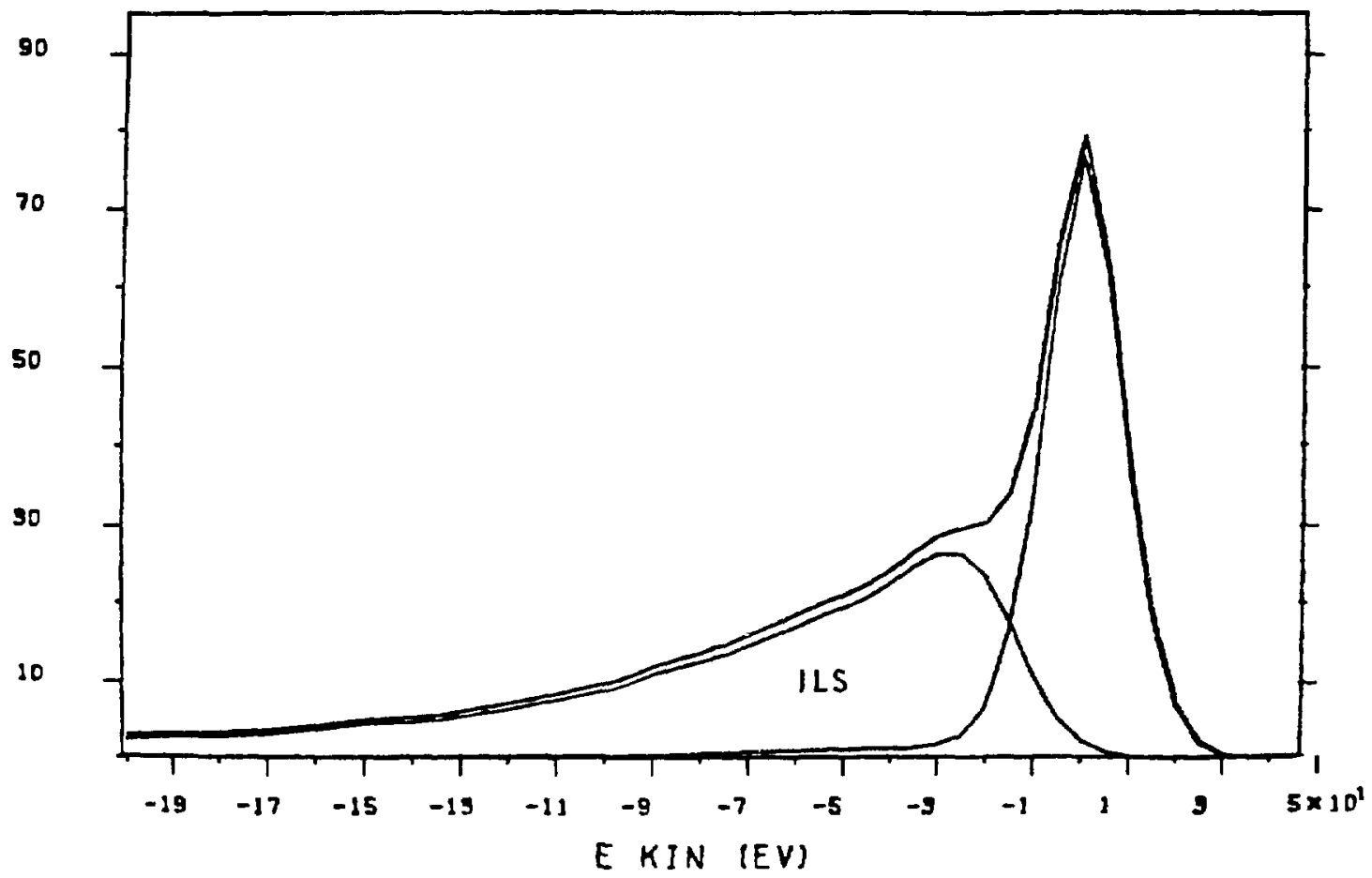

Fig. 3.1. The ITEP 1986 total resolution function. Note the width of the ionization loss spectrum (ILS). 
Implanted tritium sources have yet another problem: tritium is a very mobile atom and it is difficult to determine the profile of depths of the atoms in a substrate. During implantation of tritium ions into a crystal, some will "channel" many mean free paths deep into the crystal along the lattice. This problem can be overcome somewhat by the use of amorphous substrates, but it is still difficult to determine the distribution of tritium with depth. A similar problem faced by nonimplanted solid sources is the determination of actual source thickness. The source may be of a non-uniform thickness, making calculations of energy loss difficult.

\section{Advantages of Gaseous Tritiun Sources}

The ideal source for any experiment would be free tritium nuclei. However, a practical source made of bare nuclei has yet to be devised. Failing that, however, a gaseous source of pure tritium is the next best thing. The final states of the atoms can be easily calculated \{Wil83\}, and the spectrum for the molecular case has recently been determined by two independent groups [Mar85, Ko185]. The use of a gaseous source eliminates or reduces to a manageable level all of the problems encountered in the use of solid sources.

The dielectric constant of a low-pressure gas of tritium is essentially the same as that of a vacuum. As long as the accumulation of space charge can be eliminated, the potential of the source can be controlled very accurately. There is ionization loss in a gaseous source; however, the only mechanism for loss is inelastic scattering from gas atoms, and this has a very small cross-section when compared to the processes that can take place in a solid. The density of a gaseous 
source can be varied continuously over a wide range, allowing detailed investigation of energy loss mechanisms.

The problem of a "preferred direction" can be essentially eliminated in a gaseous source. This effect is greatly reduced by the lack of a substrate, and can be reduced much further still if the extraction from the gaseous source includes a large solid angle. If the extraction efficiency is 908 , for example, a 108 effect in the "preferred direction" (which in this case would be the direction for electrons leaving the source without being extracted) would change the extraction efficiency by only about 18 .

Since the distribution of the gas in a gaseous source is well known, there is no problem associated with the determination of the path length of any emitted beta through the gas. Thus, there is no effect comparable to the depth problem in implanted sources.

It is clear that the use of a gaseous source is the best way to provide a "clean" spectrum for determination of the neutrino mass. For this reason, the most important feature of the Los Alamos experiment is the use of pure atomic and molecular gaseous tritium sources.

\section{Design Considerations for the Los Alamos Experiment}

Although the use of a gaseous source of pure tritium greatly reduces the problems associated with interpretation of the beta spectrum, it nonetheless presents severe technical difficulties in the design of a beta decay experiment. Because tritium tends to exchange with hydrogen, a process that is catalyzed by the beta particles emitted by the tritium, the apparatus is required to be made of materials containing a minimum of hydrogen. This means that no hydrocarbons, 
including plastics or oils, could be used in the portion of the vacuum system which would see significant quantities of tritium. Even stainless steel (or any metal, for that matter) has hydrogen adsorbed into its surface, which will exchange with tritium when the gas is put into the system. This problem is much less serious than that of hydrocarbons, but is nonetheless a consideration. Teflon is also an inappropriate material for use in these areas, since the tritium will exchange with fluorine and make a gas of tritium fluoride, which is both radioactive and extremely corrosive.

Because of the low energy of the betas, the source must be windowless, which means that the gas must be continually pumped. In order to keep the inventory of tritium gas reasonably low, the gas would ideally be recirculated constantly. This, then, requires pumps with high speed and compression ratios which do not have any organic materials in them.

In order to achieve the required resolution, the potential of the source must be extremely uniform, and must be known to a high accuracy. Background can be reduced dramatically by biasing the source at a negative potential, thereby accelerating the betas between the source and the spectrometer. This raises the energy of the betas from the source above the endpoint of the betas from anywhere else in the system, so that they can be positively identified. The higher energy, however, will make the absolute experimental resolution worse, since for magnetic spectrometers the relative resolution width increases with increasing energy. The extraction from the gaseous source into the spectrometer must be very efficient in order to obtain a high enough data rate in the 
region of interest, and the betas must not have a very high probability of scattering in the source.

It is extremely difficult to make a gaseous source with a stable amount of activity, because of pressure and gas composition variations. Therefore, it is necessary to find some way to monitor the activity of the source at the same time as the data is being taken.

These difficulties are compounded when an atomic source is to be used. Atomic tritium tends to recombine very quickly on most surfaces, especially on metals. Insulators, such as Pyrex, had been tried, and performance was found to be satisfactory; however, it would be extremely difficult to control the potential of a source which has insulating walls. In addition to the determination of the total activity of a source of tritium atoms, it is also necessary to be able to measure the fraction of the gas that is atomic versus the amount that is molecular during a data run.

In order to prevent the tritium gas from entering the spectrometer, either a differential pumping system or a window of some type would be necessary. A window is impractical due to the low energy of the betas and the energy resolution necessary in these experiments. The differential pumping system must have a very low conductance for tritium gas, but a very high transmission for betas. Again this leads to the need for high-speed, organic-free vacuum pumps. In addition, however, the section of apparatus between the source and the spectrometer must emit the betas in a geometry which is suitable for entrance into the spectrometer.

The spectrometer must be insensitive to tritium that decays in the spectrometer volume, because even a very good differential pumping 
system will allow some tritium into it. In addition, it must be able to reject betas originating on or near the walls of the source. In order to obtain a high data rate, high luminosity and transmission are essential. And, of course, the spectrometer must have as good a resolution as possible.

Design of the Los Alamos Experiment

The Los Alamos experiment was designed to obtain optimal results from a gaseous source, taking into account the above considerations. The other main objective of the experimental design was to reduce background as much as possible given the need for good resolution and a high count rate. Preliminary experiments indicated that a practical flux of tritium atoms could be produced by an RF discharge in low pressure ( 1 - 10 torr) tritium gas, and, following a suggestion from D. Kleppner of M.I.T., that since there exist materials (such as Pyrex) that allow a tritium atom to bounce many times on the surface before recombining, a "bottle" could be constructed to contain enough tritium atoms to make a reasonably intense source. During the course of experiments detailed in Chapter 4, it was found that aluminum, which forms a very thin oxide layer on its surface, has excellent recombination properties. This oxide layer is so thin that electrons can easily tunnel through it, so that its potential can be very accurately defined, thereby avoiding the problems usually associated with insulating walls. A magnetic field imposed on the bottle will constrain all the electron orbits to spiral around the field lines; a high enough field will keep the electrons very near the field lines, allowing them to be "steered" away from the walls of the bottle. In 
addition, the magnetic field produces a very high extraction efficiency from the source.

A magnetic spectrometer was chosen because of the extreme sensitivity of electrostatic spectrometers to tritium decaying in the spectrometer volume, and because of concerns about other backgrounds which would be present in an electrostatic spectrometer (see Chapt. 2). The use of a magnetic spectrometer also allows the acceleration of betas from the source, which reduces the background significantly. A toroidal field spectrometer similar to the one used by Lyubimov et al. [Tre75] was chosen for its high luminosity, good resolution, and insensitivity to residual fields from the source.

The Los Alamos apparatus, then, consists of three major sections: the windowless gaseous source, a differential pumping system together with an electron transport and extraction system, and a magnetic spectrometer. When this experiment was first proposed in 1980, many considered the technical difficulties insurmountable. The problems have been overcome, however, and there is currently a working tritium beta decay experiment at Los Alamos which uses gaseous sources. A schematic diagram of the plan for this apparatus is shown in Fig. 3.2. The details of the apparatus will be covered in Chapter 4 . 


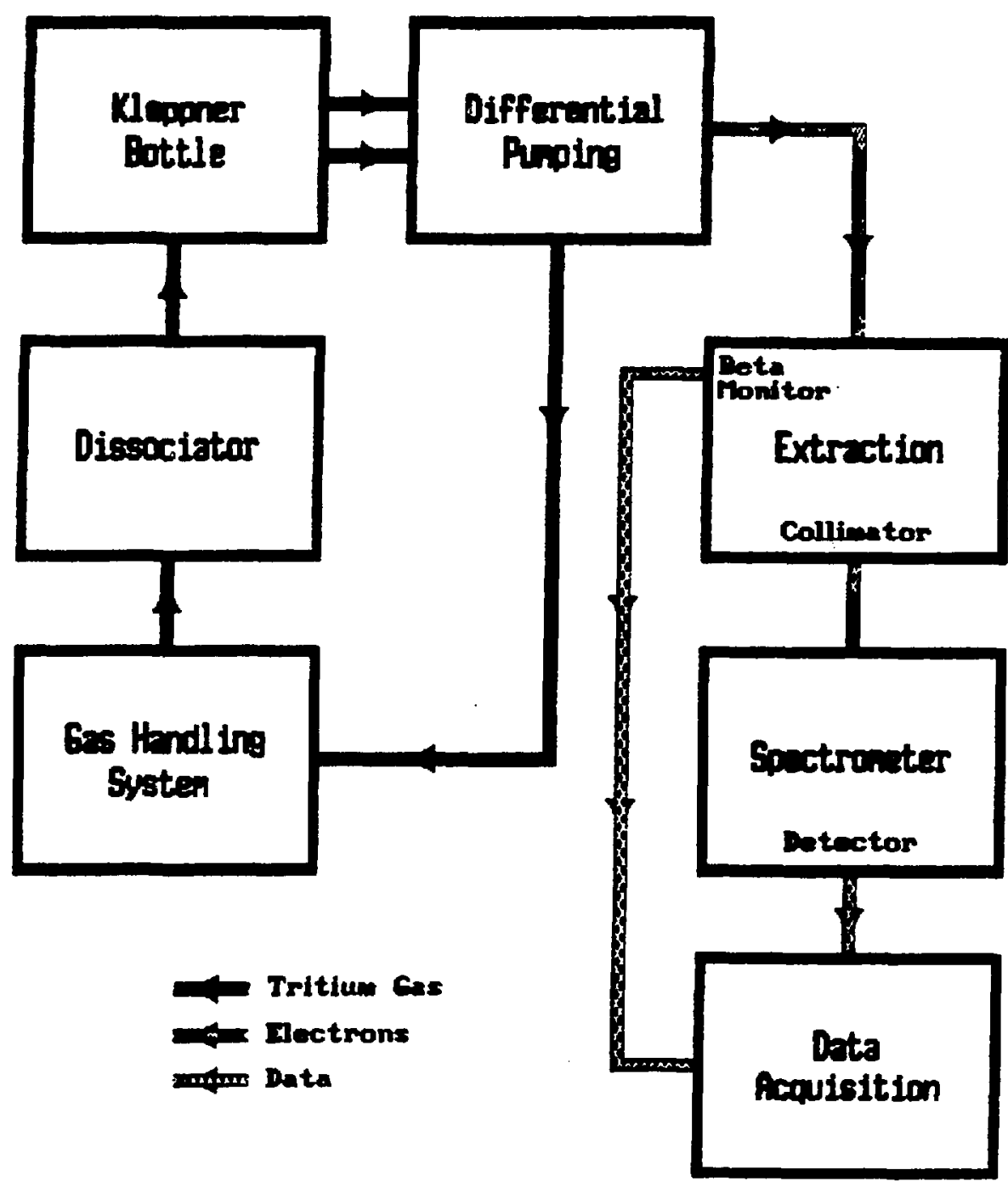

Fig. 3.2. Schematic of the Los Alamos experiment functions. 
Chapter 4:

Apparatus

\section{Overview of the Apparatus}

A cross-sectional view of the Los Alamos tritium beta decay experiment is shown in Fig. 4.1. It is quite large as beta decay experiments go, measuring more than 16 meters in length. The apparatus is divided for the purposes of this discussion into three main sections: the gaseous source, the differential pumping and electron extraction region, and the beta spectrometer. In addition to those parts of the apparatus which are shown, there is an extensive gas handling apparatus for the tritium, a data acquisition system, control electronics, and various support systems. Each of these portions of the apparatus will be described in more detail.

Tritium gas is introduced into a long tube in which it decays. The electrons are constrained by a strong solenoidal magnetic field to spiral around the field lines, which are parallel to the axjs of the tube. The electrons are accelerated by about 7 to $9 \mathrm{kV}$, and transported through a differential pumping region, which prevents the gaseous tritium from the source from entering the spectrometer. The electrons are then focused by nonadiabatic transport through a magnetic field, pass through a collimator, and are analyzed by a toroidal-field magnetic spectrometer. They are detected by a resistive-wire position-sensitive 


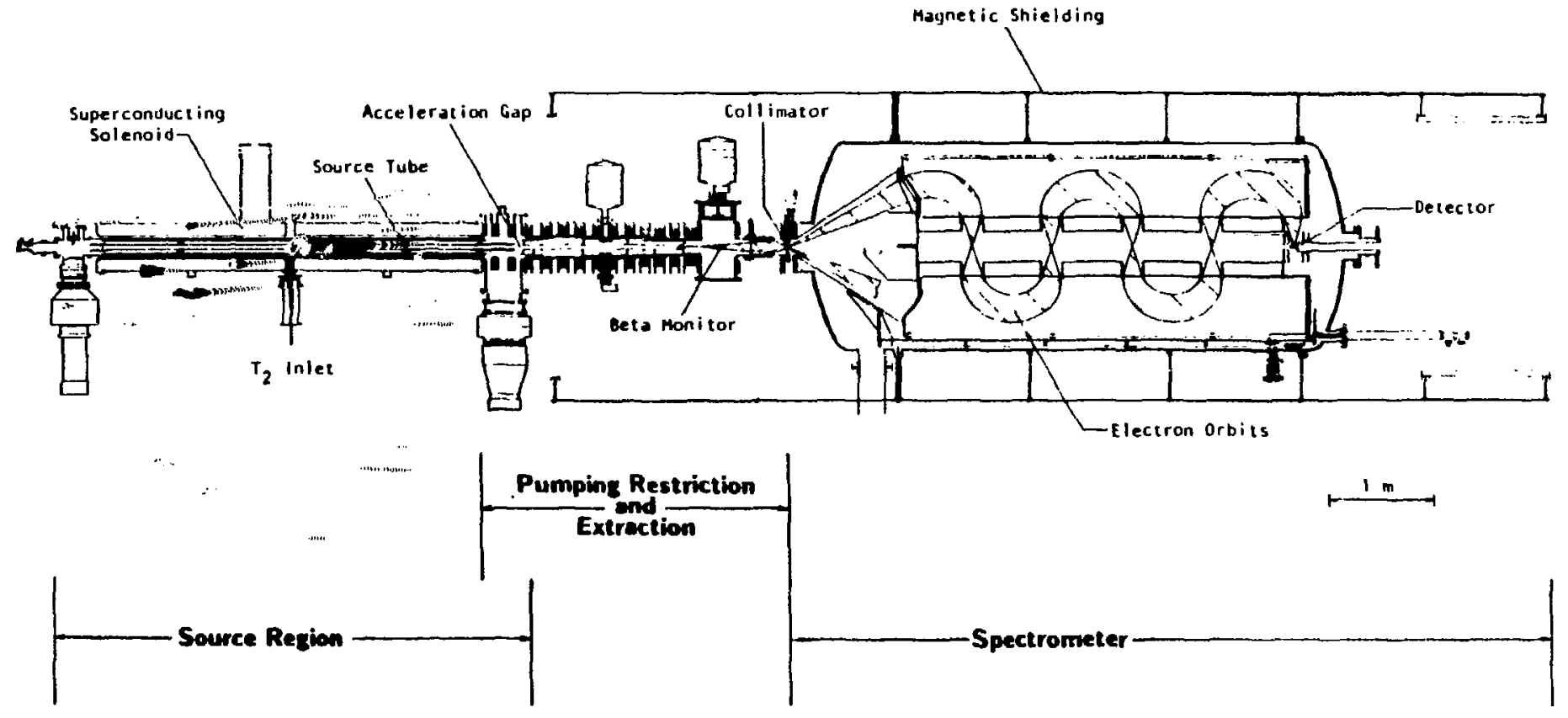

F1g. 4.1. Overview of the Los Alamos Free Atomic and Molecular Tritium Beta Decay Experiment. Major features are shown. The overall length of the apparatus is $16 \mathrm{~m}$. 
proportional counter. The total activity of the tritium in the source tube is determined by the count rate in a solid-state detector called the beta monitor, which detects electrons from the tritium decaying in the source. The apparatus has been designed to allow sources of both atomic and molecular tritium; at present, however, only data from molecular tritium has been obtained.

\section{Determination of Source Tube Material}

The primary concern in the design of the gaseous tritium source was that it be a source for atomic as well as molecular tritium. Therefore, it was necessary to design a source which would inhibit the recombination of tritium atoms into tritium molecules. This process takes place almost entirely on the surfaces of the vessel containing the tritium atoms, so it was that material which had to be selected to minimize recombination. In addition, for this experiment it was necessary that the source remain at a well-defined potential, so the ideal surface material would also be a conductor. Walraven and silvera [Wa182] had reported on an apparatus which could produce substantial fluxes of atomic hydrogen, at low temperatures. An apparatus based on similar principles, shown in Fig. 4.2, was constructed and used to test

various materials to determine the recombination coefficient $\gamma \cdot$ A tritium atom can bounce an average of $1 / \gamma$ times off the surface before recombining.

The apparatus consisted of an RF dissociator which could be cooled to-liquid nitrogen temperature $(77 \mathrm{~K})$, a tube, which could also be cooled, coated on the inside with the material being tested, a chopper, 


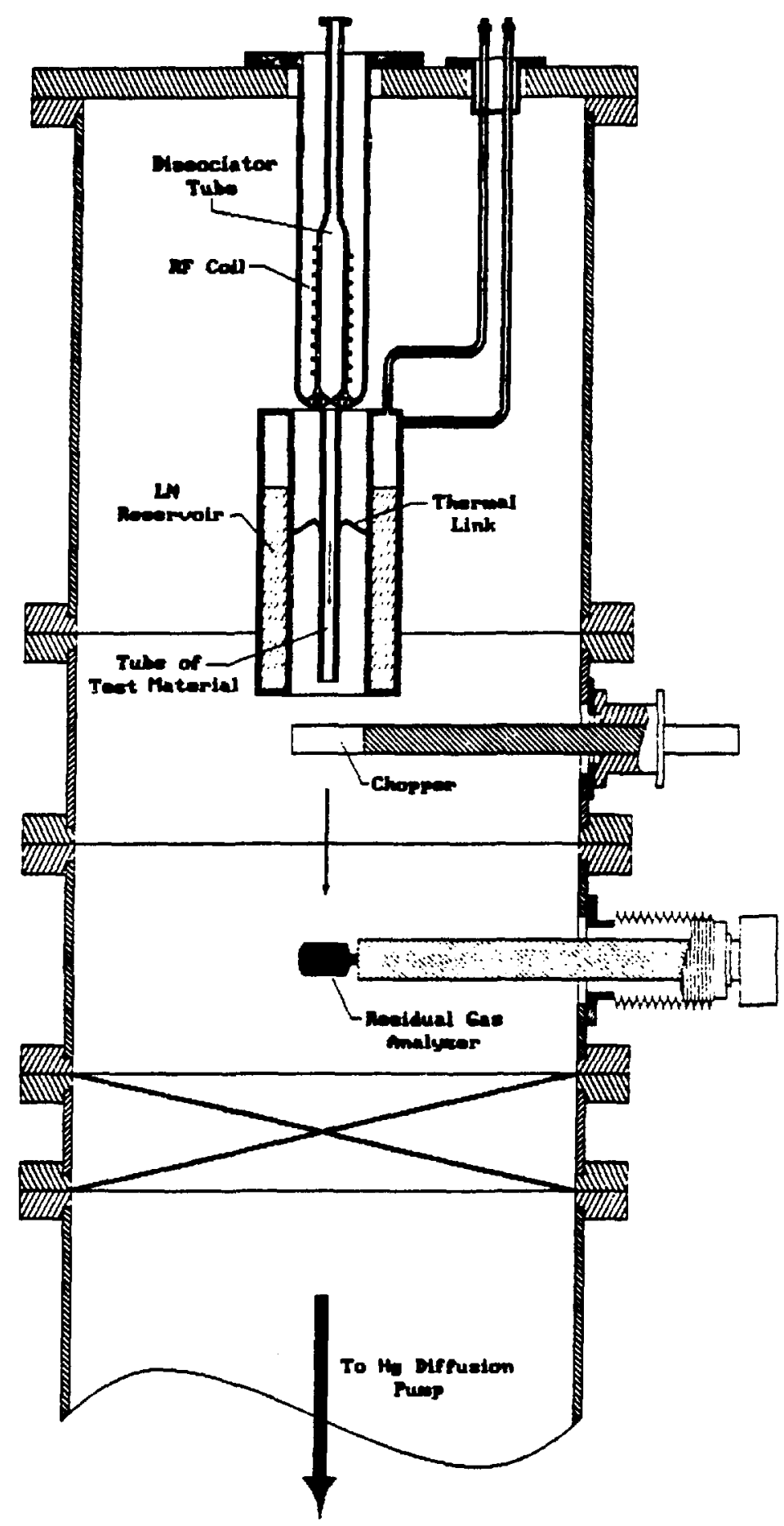

Fig. 4.2. Apparatus used for recombination experiments. Atomic tritium is generated in the RF dissociator and detected by. the residual gas analyzer. Arrows indicate the direction of gas flow. 
and a Balzers residual gas analyzer [BALO1]. The chamber was kept at a pressure, of about $1 \times 10^{-6}$ Torr by a $9^{\prime \prime}$ mercury diffusion pump. The average "number of bounces that a gas molecule makes off the walls of a tube while traversing the length of a tube is given by:

$$
\mathrm{N}_{B}=3 / 8(L / r)^{2}
$$

where $L$ is the length of the tube and $r$ is the radius. Assuming that first-order recombination processes dominate (that is, that recombination occurs between an atom "bouncing" off the wall and one trapped on the surface of the wa11), the ratio of the dissociation fraction emerging from the tube to that entering it is given by:

$$
\alpha(L) / \alpha_{0}=[\cosh (y)]^{-1}
$$

where $\alpha(z)=\mathrm{N}_{\text {atoms }} /\left(\mathrm{N}_{\text {atoms }}+\mathrm{N}_{\text {molecules }}\right)$ is the fraction of atoms at any point $z$ along the length of the tube, $\alpha_{0}$ is the initial fraction emitted from the dissociator, and $y=\left(2 \gamma \mathrm{N}_{B}\right)^{1 / 2}$.

The experiment measured $\mathrm{N}_{\text {atoms }} / \mathrm{N}_{\text {molecules }}$ with and without the dissociator power on, using a lock-in amplifier and chopper to eliminate the considerable background from the residual gas in the chamber. Measurements with no tube in place were made to determine the value of $\alpha_{0}$ for various conditions. It was found that $\alpha_{0}$ was extremely sensitive to the cleanliness of the dissociator tube and to the purity of the hydrogen gas.

The recombination coefficient $\gamma$ varies with surface temperature, due to competing processes on the surface. At high temperatures, the available chemical bonding sites on the surface are partly filled, and 
the recombination rate depends mostly on the proportion of those sites filled. In this region $\gamma$ increases with decreasing temperature, and the recombination is a second-order process (that is, it depends on the density of atoms in the tube). AC some lower temperature, the chemisorption sites are saturated, and the recombination becomes a first-order process which depends on the energy of the free atoms to excite the bound atoms on the surface sufficiently to allow recombination. In this regime, $\gamma$ decreases with decreasing temperature. Finally, at a lower temperature still, atoms begin being bound weakly to the walls by van der Waals forces (physisorption) and $\gamma$ once again increases with decreasing temperature. In Pyrex, the transition between the high temperature regimes occurs at about $550 \mathrm{~K}$, and the transition between the low temperature regimes at about $120 \mathrm{~K}$ [Wis67]. Clearly, the recombination will be a minimum at the low temperature transition.

The results of the experiment are shown in Fig. 4.3. The line for Pyrex has been taken from Wise et al. [Wis67]. As can be seen, aluminum exhibits even better behavior than does Pyrex. This is because the aluminum, unlike other metals, has a thin, impervious layer of $\mathrm{Al}_{2} \mathrm{O}_{3}$ which forms on any surface exposed to air. Other metals which were tested indicated that recombination occurred immediately on contact with the surface. The effect on $\gamma$ of the atomic weight results from the physisorption of the atoms on the walls: heavier atoms have lower velocities at a given temperature and therefore the onset of physisoxption occurs at a higher temperature. 


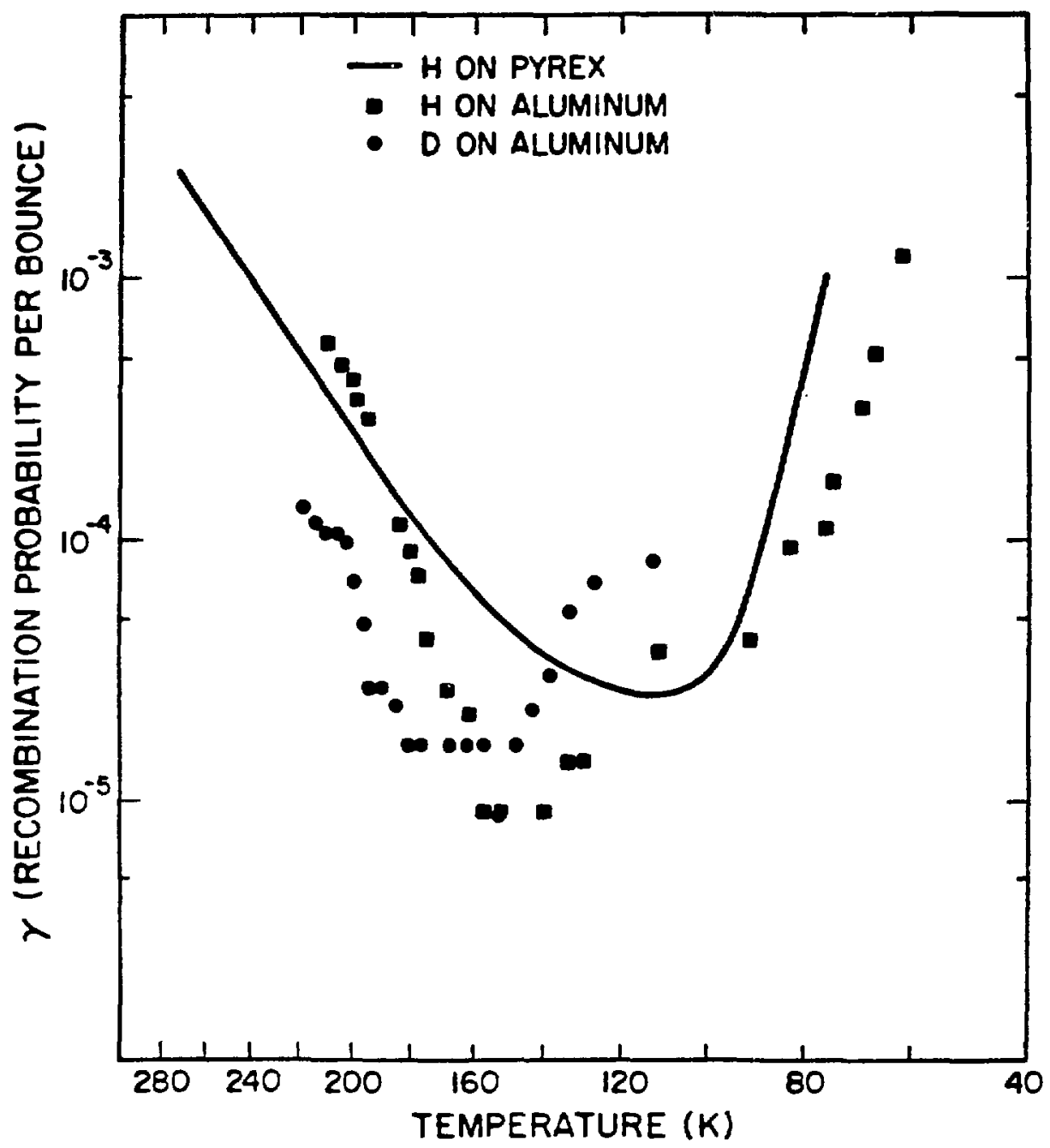

Fig. 4.3. Results of recombination experiments. Line for Pyrex has been taken from Wise and Wood [Wis69]. 
The Gaseous Tritium Source

The gaseous tritium source is shown in cross-section in Fig. 4.4. The actual source consists of an aluminum tube, $4 \mathrm{~m}$ long and $3.8 \mathrm{~cm}$ inside diameter, the inside surface of which has been polished such that the amplitude of any scratches in it is 2 microinches rms. The tube can be biased to a high potential, and its temperature can be varied by means of a temperature control system which is thermally linked to the tube through sapphire rods which are also used to support the tube. The temperature control system is shown in Fig. 4.5. The sapphire rods are clamped to the source tube by two copper rings. Each ring is cooled by a liquid nitrogen tube which passes through it and is thermally linked to it by means of an exchange gas, which is usually helium. The temperature can be controlled by altering the pressure of the exchange gas, or by means of heaters in the copper rings. The source tube wall thickness is $.64 \mathrm{~cm}$ so the temperature differences from one end of the tube to the other are minimized. The temperature of the tube can be directly monitored by means of thermocouples attached to one end; however, since the tube usually sits at a high potential, silicon temperature sensors in the copper clamps, which are at ground, are usually used to monitor and control the temperature.

Both ends of the source tube consist of a series of equipotential rings spaced $.65 \mathrm{~cm}$ apart, which maintain the potential in the center of the tube while maximizing the conductance for gas atoms to escape from the center. Near the ends of the series of rings, where the potential changes, the pressure in the center is the same as the ambient pressure 


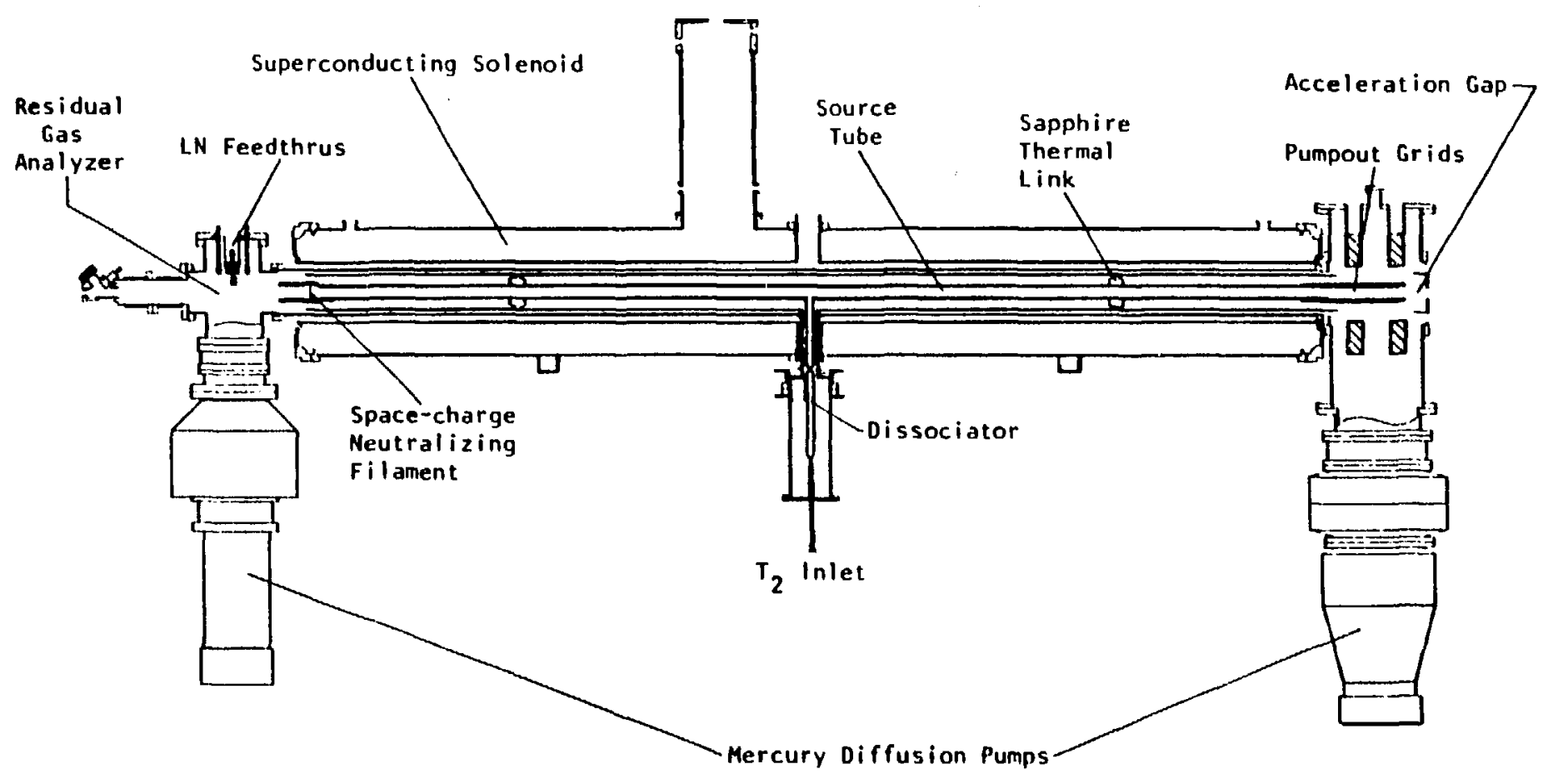

F1g. 4.4. The source region. Tritium enters through the dissociator, diffuses down the source tube, and is pumped out both ends by the nercury diffusion punps. 
of the chamber. This minimizes the distortion of the spectrum caused by tritium atoms not decaying in a well-defined potential. The negative bias of the source tube presents a potentially serious problem: the ions left behind by the tritium decay are trapped in the source, and space charge could build up and change the potential of the gas in the tube. To eliminate this problem, two tungsten filaments were strung across the center of the tube at the position of the pinch in the magnetic field. One of these filaments is heated until thermionic emission can occur. The low-energy electrons from these filaments then expand into the entire volume of the source tube and neutralize the space charge. Any extra electrons are accelerated out of the tube at the ends. The system is self-rogulating: if the space charge builds up, the emission current increases, and only the electrons which are needed to neutralize the space charge remain in the tube.

The electrons are accelerated at the end of the rings between the source tube and the pumping restriction. Great care must be taken at this point to keep both the electric and magnetic fields cylindrically symmetric about the same axis, in order to prevent the $\mathbf{E} \times \mathbf{B}$ force from changing the average radius of the beta particles from the axial center of the fields. This gap is one of the areas of the apparatus most susceptible to a high voltage breakdown because the electric and magnetic fields are parallel. Therefore, great care was taken to polish all the surfaces involved to prevent is:ona discharge.

The dissociator assembly is shown in Fig. 4.6. The tritium molecules are dissociated in an RF discharge which is induced by a coil surrounding a glass tube containing the tritium. The tritium atoms then 


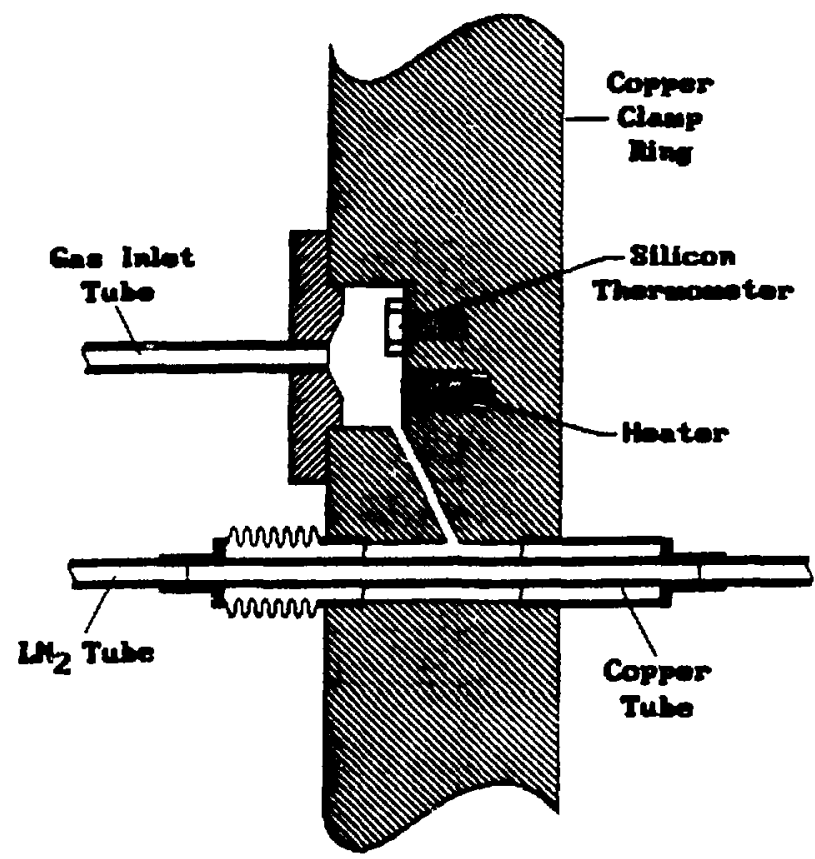

Fig. 4.5. The temperature control system for the source tube. The pressure of the exchange gas, the flow rate of liquid nitrogen through the tubes, and the power to the heaters can all be adjusted.

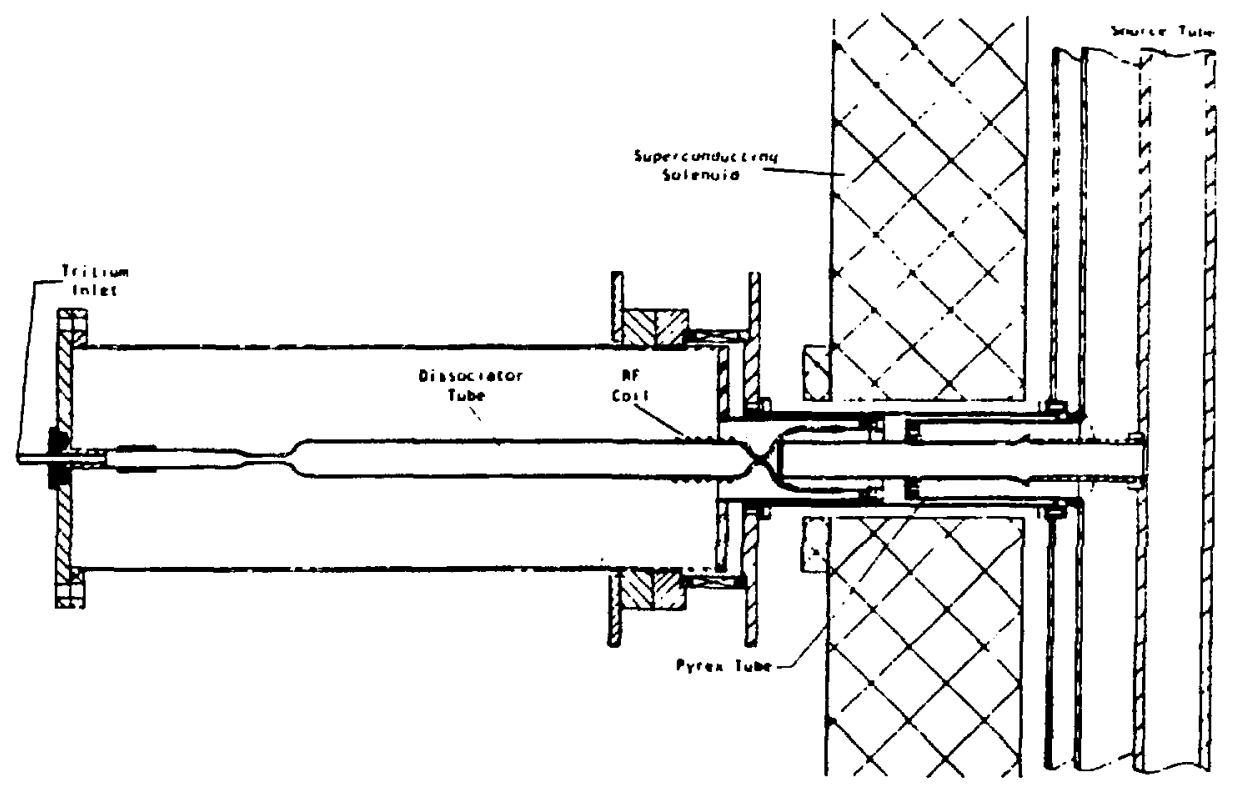

Fig. 4.6. The dissociator and side penetration into the source tube. 
diffuse through a small orifice in the end of the tube, and into the transfer region between the dissociator and the source tube. The size of the orifice is determined by the pressure needed in the dissociator to maintain a good discharge and the total flux of tritium atoms desired. In order to keep the atomic fraction high at the entrance to the actual decay region, it was necessary to ensure that the only wall materials seen by the atoms were aluminum and Pyrex. The potential of the atoms changes along this transfer region, hence the need for Pyrex. Crevices can trap tritium atoms and cause recombination, so they were eliminated by using a series of knife-edge seals between the aluminum and Pyrex. The dissociator must be kept in a sealed environment so that a break in the glass tube will not leak tritium into the surrounding air. Therefore, the RF circuit used in the dissociator can be tuned through a feedthru, and the air contained in the volume surrounding the dissociator is circulated to provide cooling for the RF coil and tube, which dissipate about $20 \mathrm{~W}$.

The entire source is kept in a solenoidal magnetic field which is provided by a superconducting solenoid [CCLOI]. The magnet was made superconducting to save money, since the power available to the building in which the experiment is housed is insufficient to provide the needed magnetic field with normal coils, and the cost of adding power would have outweighed the additional costs incurred in making the coils superconducting. The field can be shaped so that there is a constant gradient from one end to the other, which prevents stationary orbits; however, this feature has not yet been implemented. The solenoid has a pinch coil at one end that reflects most of the betas with initial 
momenta pointed into the half of phase space toward that end of the source. When the solenoid is configured for a uniform field of $1.5 \mathrm{kG}$, the field is constant to within 30 gauss along its length. The measured field profile for a constant current of $49.5 \mathrm{~A}$, corresponding to a nominal field of $3.1 \mathrm{kG}(0.31 \mathrm{~T})$ is shown in Fig. 4.7. The magnet was built as one piece, with side penetrations consisting of two $8 \mathrm{~cm}$ diameter radial holes in the center. The dissociator assembly passes through one of these, and the other is used for forced air circulation to prevent the bore of the solenoid and the vacuum tube around the source from getting too cold, a condition which would stress vacuum seals.

A significant adjunct to the source is the gas handling system. A schematic of this system is shown in Fig. 4.8. When running, the tritium is recirculated, thus minimizing the necessary inventory. The tritium is purified by diffusion through palladium metal heated to $350^{\circ} \mathrm{C}$, which only allows the passage of hydrogen isotopes. The tritium then enters the dissociator, and then the source tube. The gas is mostly recovered by the mercury diffusion pumps [EDW01] at either end of the source, with a small amount pumped by the cryopurips in the pumping restriction. The diffusion pumps are backed by a special booster diffusion pump [EDW02] which is capable of a fore pressure of about 20 Torr. The gas then passes through a series of traps and filters designed to prevent mercury from spreading throughout the system, and it is reintroduced into the high-pressure side of the palladium leak.

Non-hydrogenic gases, such as nitrogen and ${ }^{3} \mathrm{He}$, are trapped at the high pressure side of the palladium leak, After a time, they can 


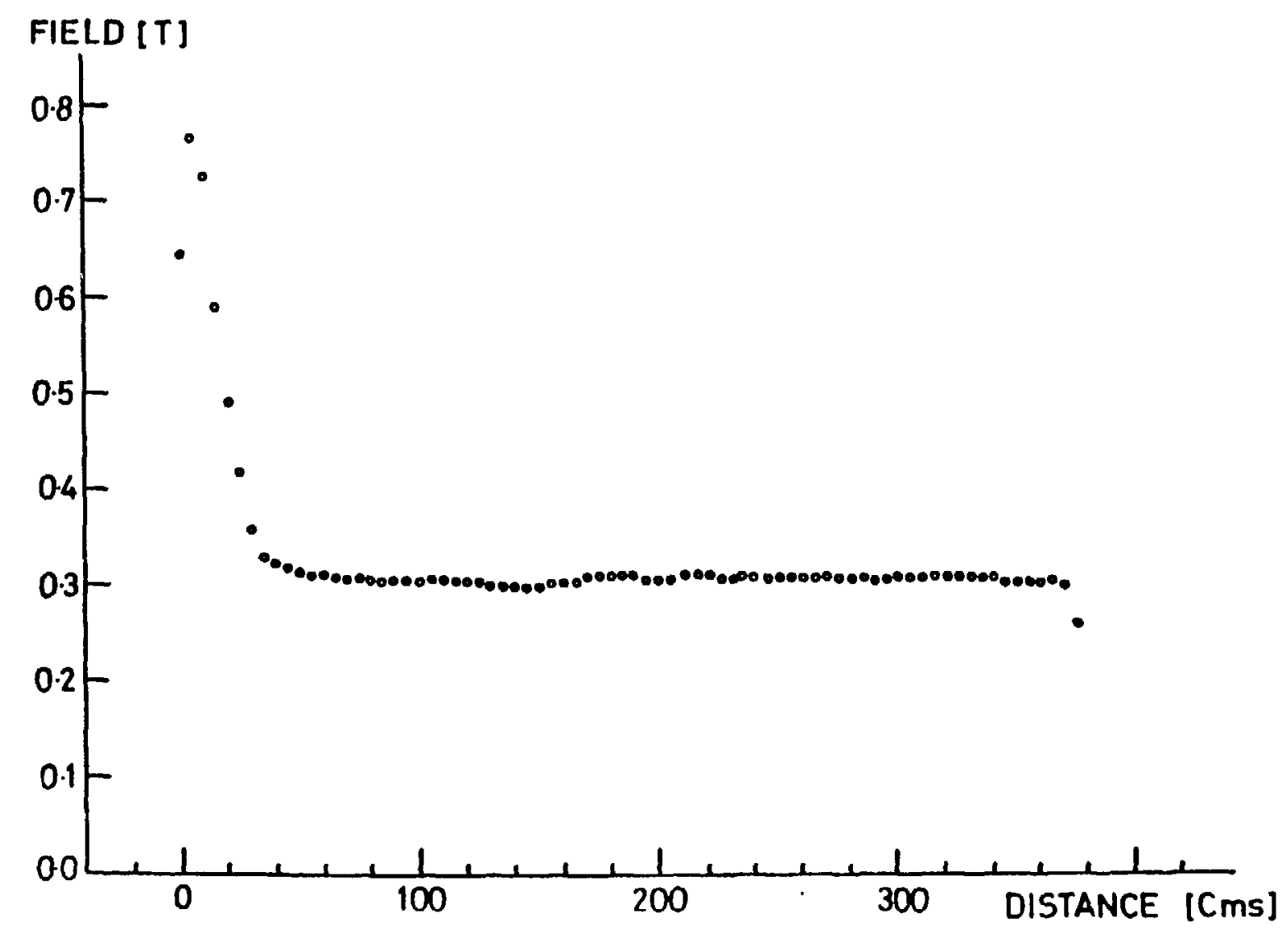

Fig. 4.7. Field profile of the superconducting solenoid, measured by Cryogenics Consultants, Ltd. The field was configured to be constant with a pinch at one end. In actual use, the magnet is run at $1.5 \mathrm{kG}$, with a pinch of $4 \mathrm{kG}$ at one end. 
IPITIUM GAS HANOLNG SYSTEM

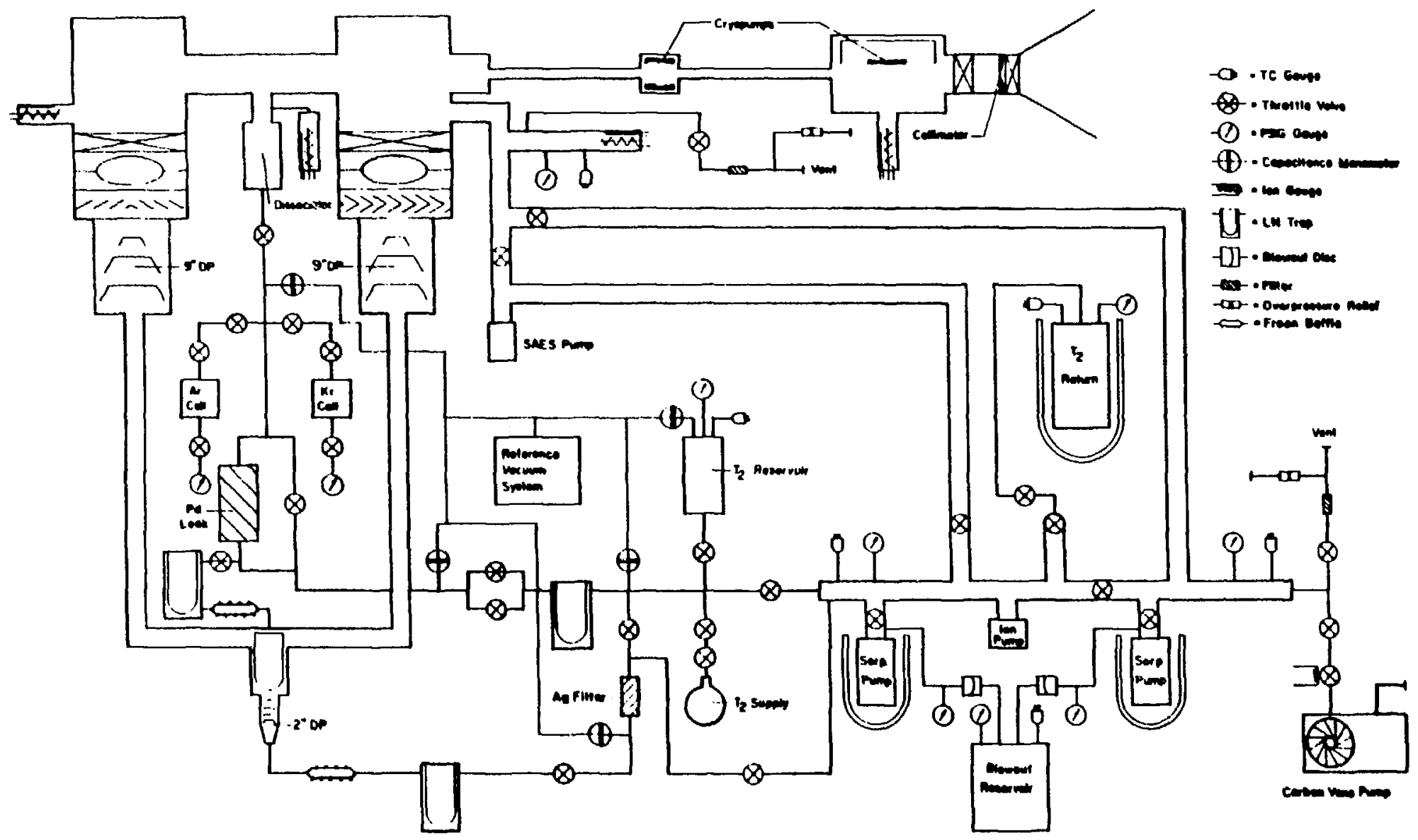

Fig. 4.8. The tritium gas handling syster. 
prevent the tritium from diffusing to the leak quickly enough to maintain an adequate pressure in the source. Therefore, these gases are recirculated by a secondary system which bypasses the source and is pumped by the booster pump. The tritium can be stored between runs in a regenerable getter pump [SAE01]. While this pump does not pump helium, it does pump nitrogen and oxygen (and is nonregenerable for those gases) so only relatively pure tritium can be stored in this manner.

An important tool in the source region is a Balzers residual gas analyzer [BAL01] which allows an analysis of the composition of the gas in the source. It is used to determine the purity of the tritium gas, in order to see any exchange that has occurred with hydrogen and deuterium in the system (see Chapt. 3). It can be used to monitor the amount of ${ }^{3} \mathrm{He}$ buildup in the tritium, although it is necessary to bypass the Pd leak to make this measurement.

A ${ }^{103} \mathrm{Ru}$ conversion-1ine electron source on a solid substrate can be inserted between rings at the end of the source near the acceleration gap (Fig. 4.9). It allows tests of extraction efficiency and spectrometer transmission. The source is mounted on an external manipulator which allows it to be inserted between and extracted from the rings while the system is under vacuum.

\section{The Pumping Restriction and Extraction Region}

The tritium pressure at the acceleration gap is normally about $1 \times 10^{-5}$ Torr, and this must be reduced by at least four orders of magnitude at the entrance to the spectrometer. The differential pumping required for this is performed by the pumping restriction, shown in Fig. 


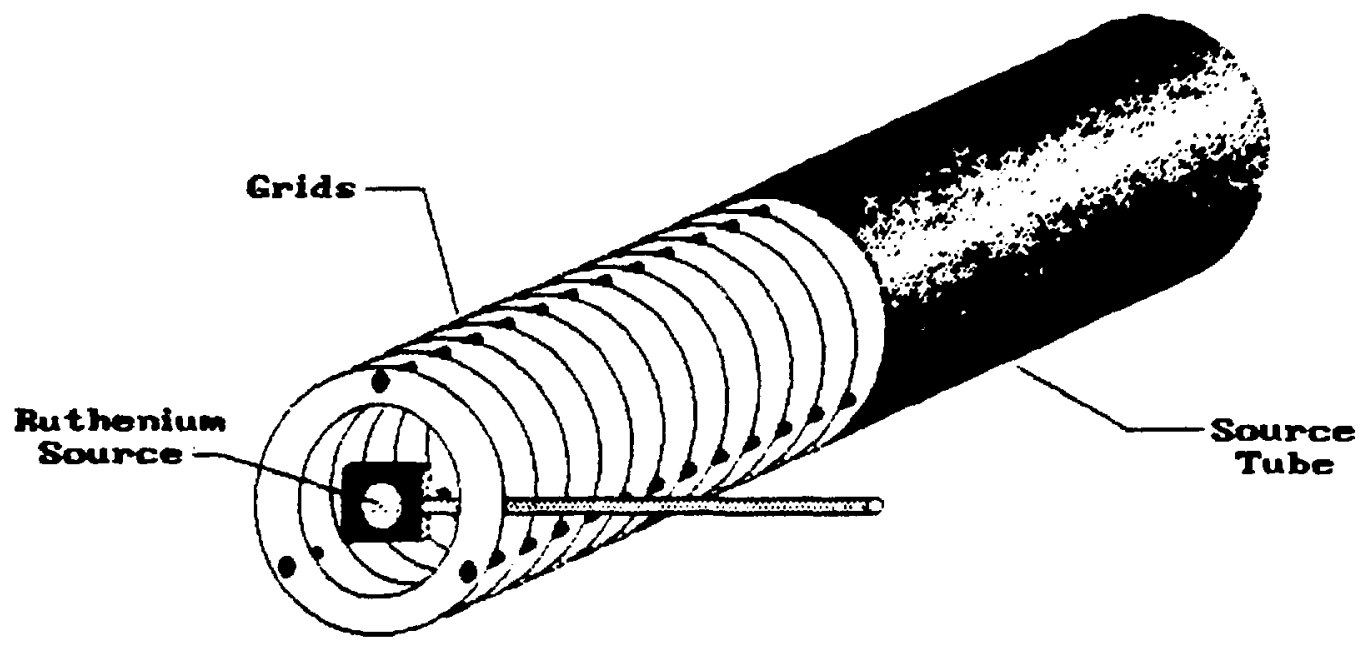

E1g. 4.9. Location of the Ruthenium source between the rings at the end of the source tube. The rings extend beyond the length shown.

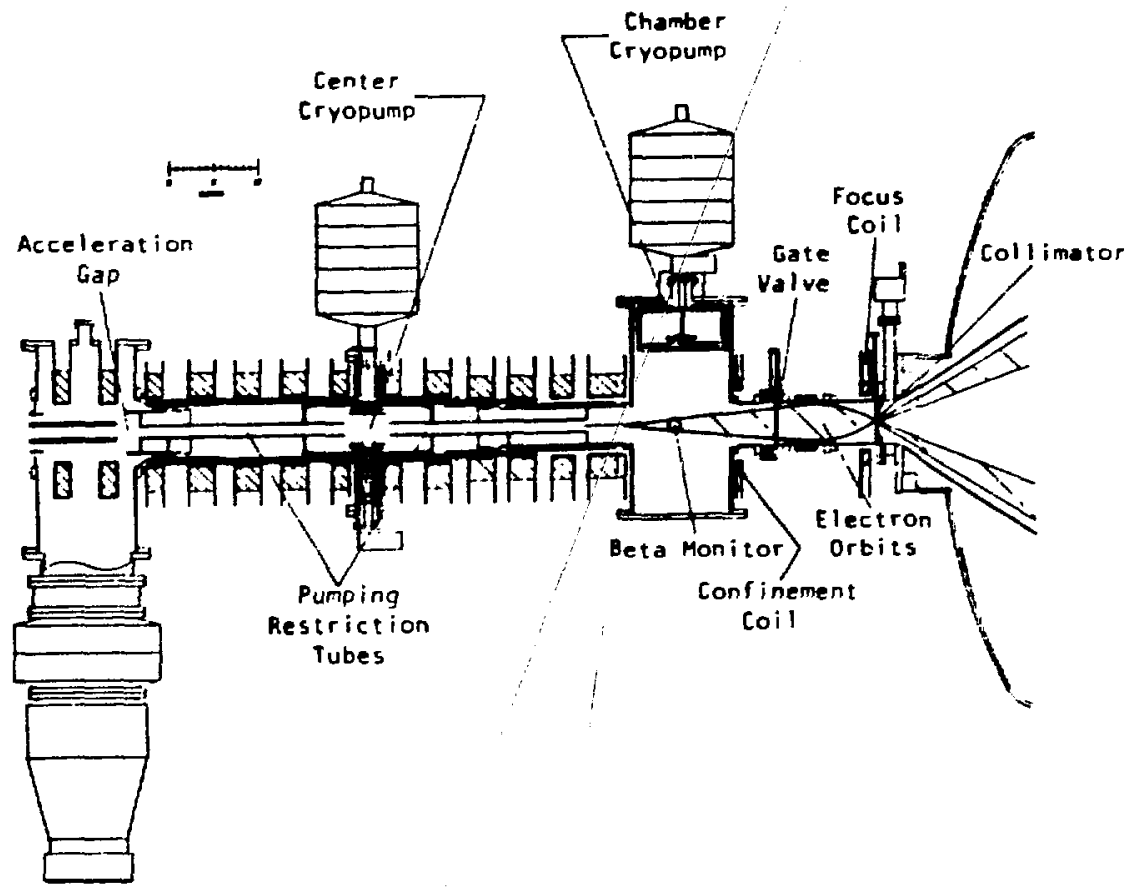

Fig. 4.10. The pumping restriction and extraction region. The envelope of electron orbits is shown. 
4.10. It consists of two $3.8 \mathrm{~cm}$ ID copper tubes, each about $60 \mathrm{~cm}$ long, which are cooled to $65 \mathrm{~K}$, with a cryopump between them and a cryopump after them, but before the entrance to the spectrometer. For low-pressure gases in the molecular flow regime (at pressures less than about 20 millitorr) the conductance of a cylindrical tube is given by:

$$
F=3.638 \mathrm{kA}(\mathrm{T} / \mathrm{M})^{1 / 2} \ell / \mathrm{sec} \text { [Dus49], }
$$

where $A$ is the cross-sectional area of the inside of the tube in $\mathrm{cm}^{2}, T$ is the temperature of the gas in Kelvin (in general this is the same as the temperature of the tube), and $M$ is the molecular weight of the gas [Dus62]. $\mathrm{K}$ is the Clausing factor, which is a function of the aspect ratio $L / a$, where $L$ is the length of the tube and $a$ is the inside radius. For the tubes in the pumping restriction, the conductance is about 10 $\ell /$ sec. The actual conductance is slightly higher due to higher temperature gas molecules that can go straight down the tubes without scattering from the walls. This process adds less than $1 \ell / \mathrm{sec}$ to the conductance.

The cryopumps were made specifically for this experiment, since it is required that they contain no hydrogenous materials. In both cases, the pumping surface is charcoal which has been glued to copper with sodium silicate. The pumping surfaces are cooled to about $13 \mathrm{~K}$ by closed-cycle helium refrigerators [CTIO1]. The refrigerators also cool a radiation shield around the pumping surface to about $65 \mathrm{~K}$, and the pumping restriction tubes are also cooled by the same refrigerator which cools the center cryopump. 
Both cryopumps were designed to have a high pumping speed for tritium gas, and therefore the highest possible conductance to the pumping surface. The center pump is built in a hexagonal shape with the pumping surface facing toward the center of the tubes. The chamber cryopump, shown in Fig. 4.11, has baffles which the gas must traverse in order to reach the cold surface, so it will not have as high a pumping speed. The baffles are necessary, however, in order to cool the pumping surface to the required temperature. Both pumps have a liquid nitrogen shield around the outside. The $\mathrm{LN}_{2}$ shields provide the ability to cool a large mass to $65 \mathrm{~K}$, as is required for the pumping restriction tubes, and they keep the radiation loads low enough to enable the refrigerator to maintain the temperature of the pumping surfaces at around $13 \mathrm{~K}$. In a conventional cryopump without the $\mathrm{LN}_{2}$ shield, the pumping surface is at about $15-20 \mathrm{~K}$, a temperature which is too high to effectively pump large volumes of hydrogen isotopes. The inside surfaces of all the shields have been painted black with pure carbon, reducing the heat load to the pumping surfaces caused by reflection of infrared radiation emitted from higher temperature surfaces.

In addition to being used for differential pumping, this section of the apparatus is used to extract the betas from the solenoidal magnetic field of the source into the toroidal magnetic field of the spectrometer. This is accomplished by nonadiabatic transport of the electrons through a solenoidal magnetic field whose axial component changes sign. Nonadiabatic transport is required to separate the electron orbits from the magnetic field lines; otherwise, the orbit radii would grow inversely as the square root of the magnetic field. 


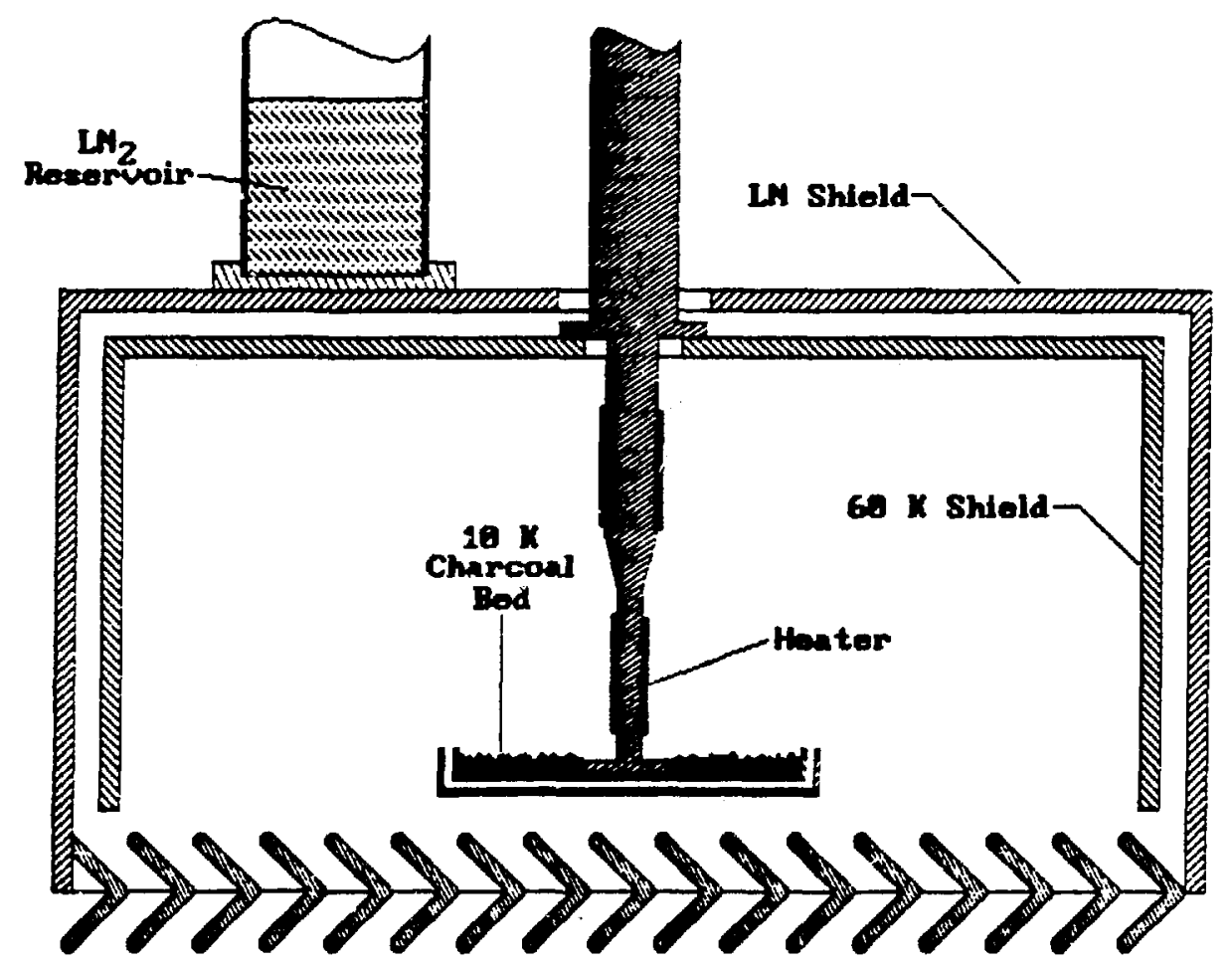

Fig. 4.11. The chamber cryopump. 
The betas are focused to a $1 \mathrm{~cm}$ diameter collimator in such a way that the electrons originating in the center of the source tube are focused while those from nearer the walls are defocused and cannot pass through the collimator. The z-component of the magnetic field (which is the component of interest) is shown in Fig. 4.12a, and the calculated orbits are shown in Fig. 4.12b. The transport of electrons through this kind of field cannot be calculated analytically, so the optimal configuration was determined by numerical ray-tracing. The field has several features of interest. At the end of the second pumping restriction tube, the magnetic field drops off suddenly, a result of a "bucking coil" placed against the end of the last coil responsible for the solenoidal field. The bucking coil has a field direction opposite that of the solenoidal coils, and it therefore reduces the field suddenly. It is in this region that most of the nonadiabatic behavior takes place. On the other side of the cryopump tank, a small coil is placed to keep the field at about $40 \mathrm{G}$, which prevents the electron orbits from striking the walls of the vacuum chamber. This coil is known as the confinement coil. Finally, a coil is located almost directly around the collimator. Like the bucking coil, the direction of its axial field is opposite to that of the other coils. The electrons are focused to an image close to the point where this negative field reaches its maximum. After the focus coil, the field drops off very rapidly, and even changes back to a positive sign as the residual field from the solenoid overwhelms the residual field from the focus coil. The coils which provide this field are not superconducting, and therefore require a significant amount of power. They are water-ccoled by a chilled water system which also is 


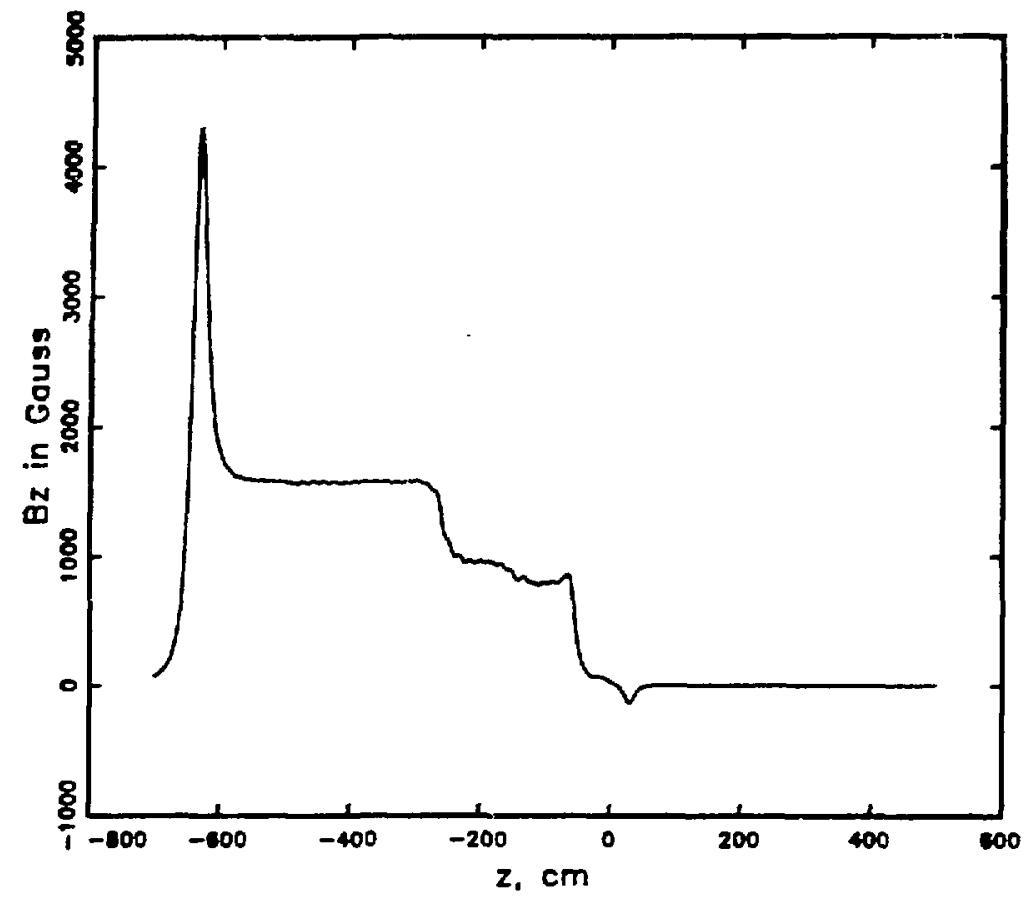

FIg. 4.12a. The $z$ component of the magnetic field in the apparatus. The collimator is located at $z-34.3$.

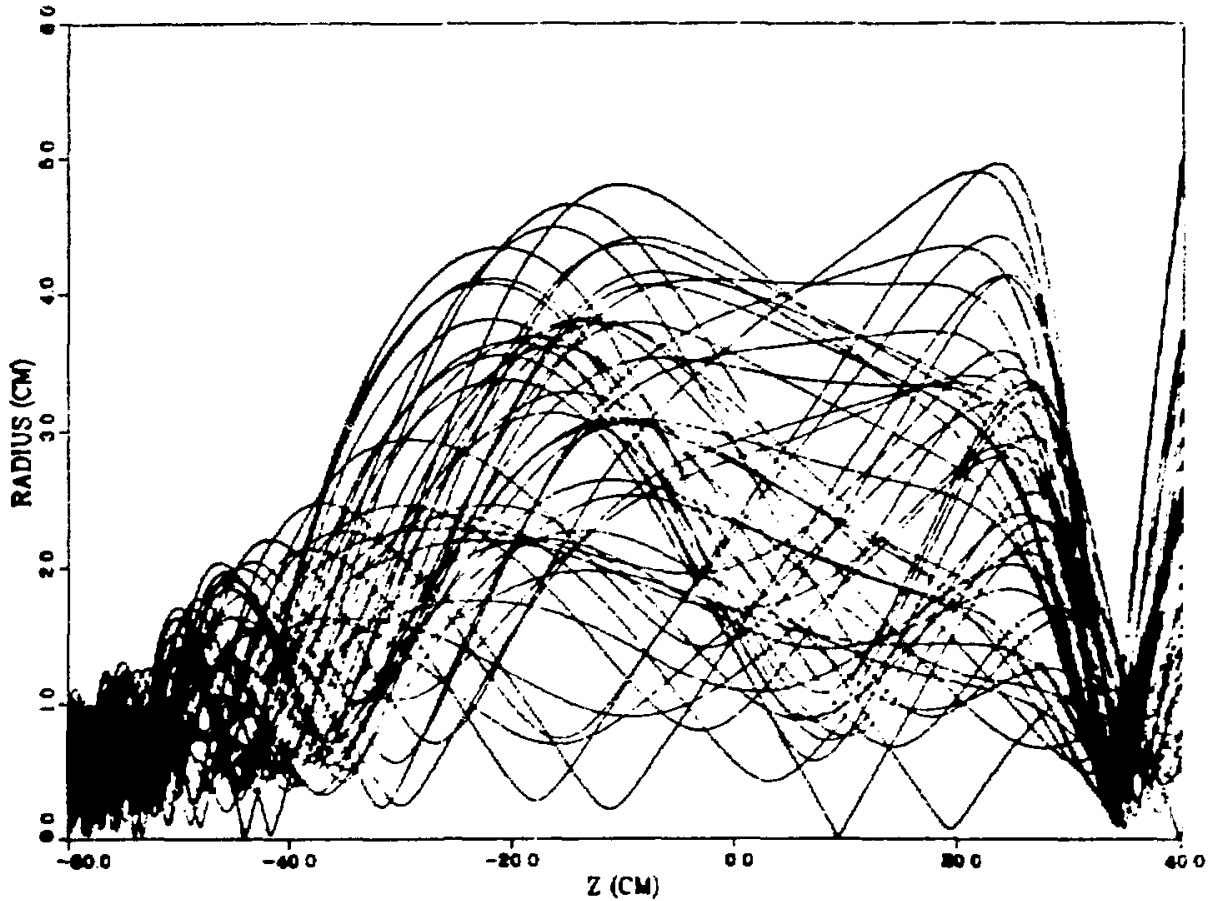

FIB. 4.12b. Calculated electron orbits in the extraction region. The collimator is located at $z=34.3$. 
used to cool the spectrometer and the diffusion pumps. The control system for these magnets is shown in Fig. 4.13. The currents in the coils responsible for the extraction can be varjed by about 10 to allow optimization of the ixtraction efficiency for the electron energy that is being used. The currents are monitored by measuring the voltages across high-precision resistors, which are water-cooled to maintain a stable temperature.

In the center of the cryopump tank, after the pumping restrictions, is a solid-state beta detector which is used to determine the source strength. This detector is located about $4 \mathrm{~cm}$ off-axis, in a portion of the electron beam which would not be accepted into the spectrometer, but which originates between the source volume and the wall of the source tube. The beta monitor and associated electronics is floated at the same potential as the source tube, so that the electrons are decelerated before entering the detector. This prevents the count rate of low energy electrons (from the space-charge neutralizing filaments, for example) from completely flooding the detector.

\section{The Beta Spectrometer}

The electrons which pass through the collimator are analyzed by a magnetic spectrometer. The spectrometer is an iron-free toroidal field spectrometer with third-order corrections to the entrance and exit apertures which allow it to accept a large fraction the beta rays from the gaseous source without significant degradation of resolving power. The geometry of this type of spectrometer is shown in Fig. 4.14. The dispersion is along the axis of symmetry (the z-axis). The electron 


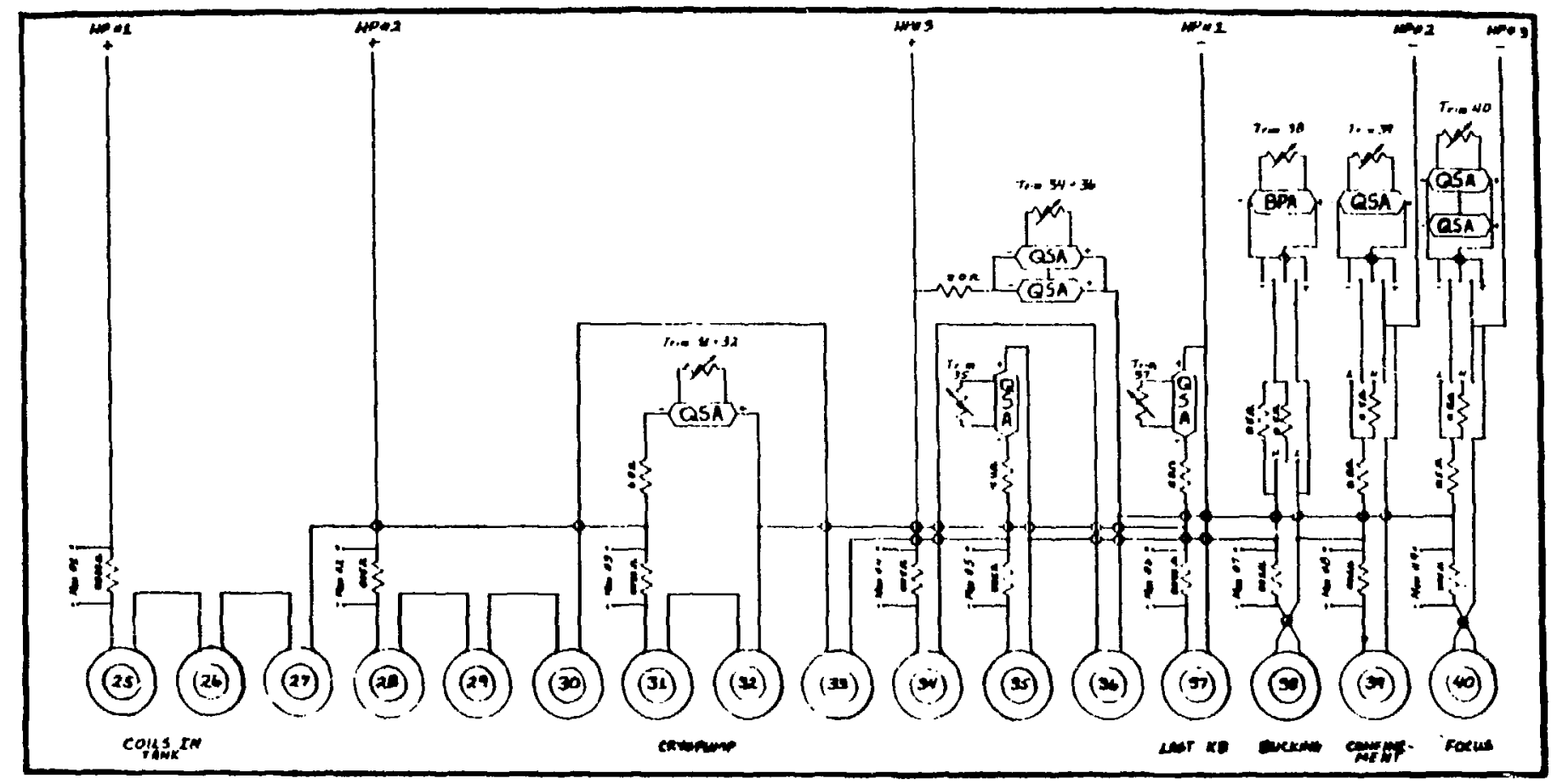

MOMINAL_CURRENTS

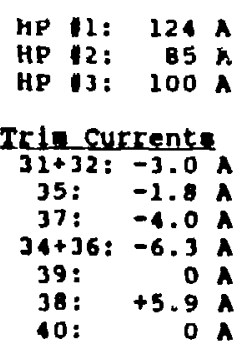

\begin{tabular}{|c|c|c|c|c|}
\hline \multirow{3}{*}{ LoCATION } & $40 \mathrm{~N}$ & VOLTAGE & RESISTANCE & POWER $\mathrm{Y}$ \\
\hline & 1 & .113 & .00333 & $\mathrm{HP} I 1$ \\
\hline & 3 & -.125 & .005 & HP 12 \\
\hline \multirow[t]{3}{*}{ Cryopunp } & 3 &. .403 & .00333 & HP IIiTrl- $31+32$ \\
\hline & 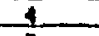 & .169 & .005 & HP 3 itrin $34+36$ \\
\hline & 5 & .416 & .005 & HP IRTTEI 35 \\
\hline Last $K B$ & 6 & -.400 & .00333 & HP UiTrIL 37 \\
\hline Bucking & 7 &. .154 & .005 & HP 12:Trin 32 \\
\hline Contine & 8 & .125 & .005 & HP IRTrin 39 \\
\hline Focue & 9 & .500 & .005 & HP IJiTrin 40 \\
\hline
\end{tabular}

Fig. 4.13. Control electronics for the extraction region magnets. 
orbits pass from the region inside the coils to a field-free region in the center of the torus several times during their transit through the spectrometer. The design is similar in concept to that of the Tret'yakov spectrometer [Tre76]; however, there are significant differences between the devices. The most novel feature of the los Alamos instrument is its entrance aperture. The Tret'yakov spectrometer accepts electrons from a solid source at a natural focal point, one of the foci seen in the field-free region along the axis of the instrument. These electrons enter the magnetic field at a nearly normal angle of incidence. The Los Alamos instrument accepts electrons extracted from a gaseous source which enter the magnetic field region at much lower angles. A comparison of the orbits of electrons in the Tret'yakov and Los Alamos spectrometers is shown in Fig. 4.15. A more detailed view of the Los Alamos spectrometer is shown in Fig. 4.16. The entrance and exit conductors in this spectrometer are shaped in order to correct for third-order aberrations and to maximize the luminosity given this geometry. In order to maximize the transmission through this spectrometer, the "crossing conductors" -- those parts of the inner conductors through which the electron $:$ bits pass - are only $.05 \mathrm{~cm}$ thick. The spectrometer is also much larger than the Tret'yakov instrument. The spectrometer volume is pumped by three vacuum pumps: a turbomolecular pump, a cryopump, and a titanium sublimation pump. The cryopump is used to maintain a good vacuum generally, while the titanium sublimation pump pumps any tritium entering the spectrometer volume from the pumping restriction very efficiently, keeping it on cooled walls near the entrance, away from the detector. The turbomolecular pump is 


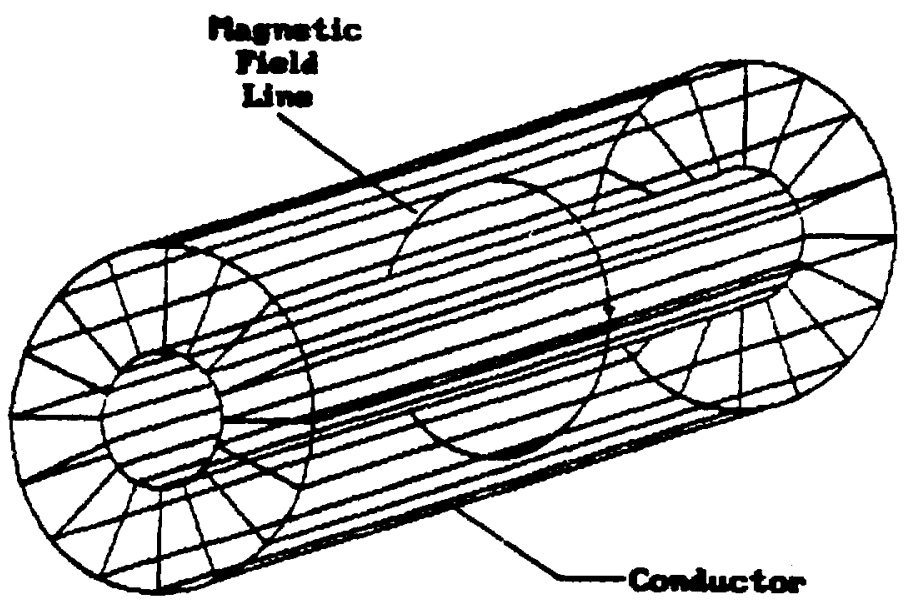

Fig. 4.14. Geometry of a toroidal spectrometer. The direction of the magnetic field lines is shown.

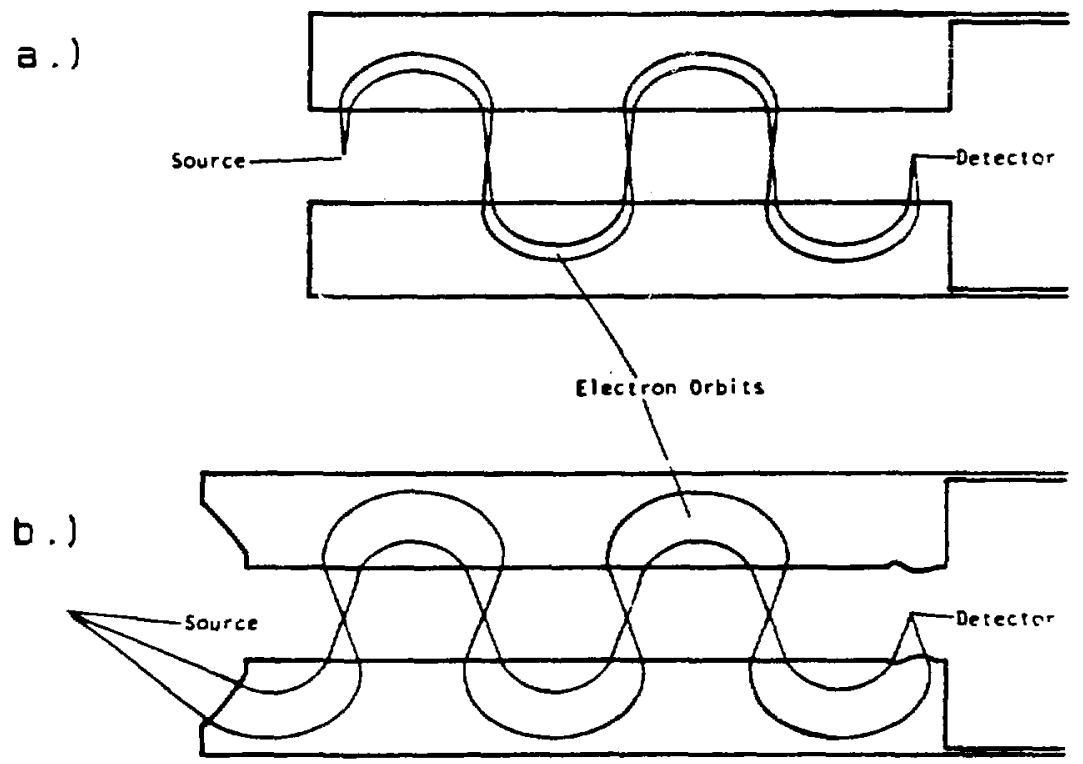

FIg. 4.15. A comparison between the Los Alamos and the ITEP spectrometers. a) the ITEP spectrometer. b) the Los Alamos spectrometer. 


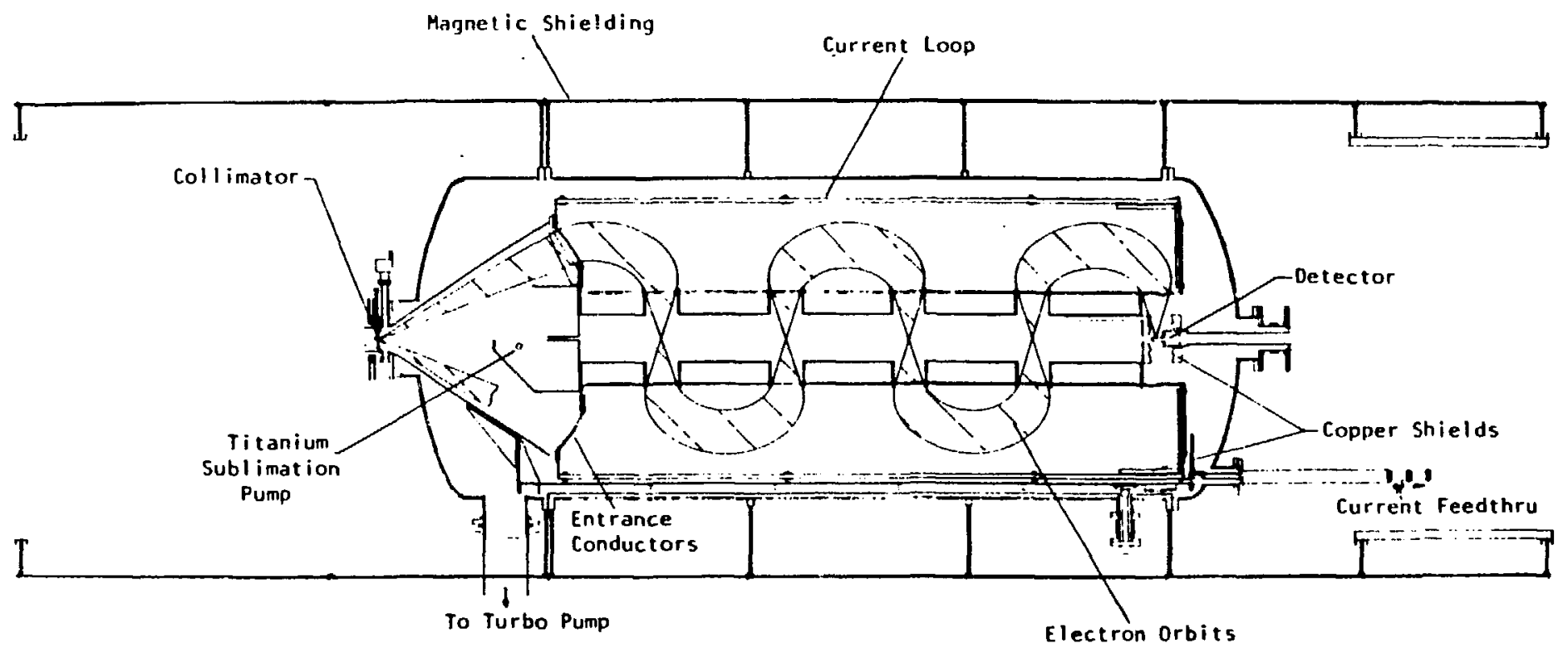

Fig. 4.16. Detail of the Los Alamos spectrometer. 
used during the operation of the experiment to remove helium gas from the system, since no other pump can effectively do so.

In order to achieve the necessary resolution, the Earth's magnetic field must be canceled inside the spectrometer volume. Helmholtz coils of the required size would be impractical, and it is not necessary to cancel the field as well in the $z$ direction as in the radial direction, since (to first order, at least) the main effects of an axial field are to induce precession of the orbits around the axis and to lengthen the focal distance slightly. Therefore, a set of $\cos \theta \operatorname{coils}$ were constructed surrounding the spectrometer. This geometry has the advantage of a very uniform field inside a large fraction of the volume occupied by the coils. The building surrounding the spectrometer was degaussed as fully as possible, and extensive field maps of the spectrometer volume determined that field gradients (which cannot be canceled by this type of coil) were acceptably small, after a compensation coil to eliminate a vertical gradient in the horizontal field was added. The mean value of the Earth's field in the building is $430 \mathrm{mG}$ with the compensation coils turned off; with the shielding on, the mean value in the spectrometer is set at zero with the fjeld at any point no higher than $20 \mathrm{mG}$ in the radial direction. Magnetometer probes were installed at the center of the spectrometer, in the field-free region, to monitor variations in the Earth's field and to allow active feedback to the magnetic shielding. This feature has thus far proved to be unnecessary.

The electrons are focused onto a detector, as shown in Fig. 4.16. The focus is not a point, but rather a ring $2 \mathrm{~cm}$ in diameter. Since the dispersion is along the axis of symetry, the detector is designed to 
accept electrons from any radial direction. To minimize backgrounds, the spectrometer has several large copper shields situated so that the only material seen by the detector is copper at least 1 inch thick. These shields are shown in Fig. 4.16. For the first set of measurements, a resistive-wire proportional counter was used, the position of the electron along the wire being determined by resistive division. A schematic of the active portion of this detector is shown in Fig. 4.17. Because the position resolution of the detector is not sufficient to determine energy from the recorded position along the wire, a $2 \mathrm{~mm}$ wide window was used to keep the momentum spread of the incoming electrons small. The position information was used primarily to reduce the background. The material for the window was .25 mil thick $\left(450 \mu \mathrm{g} / \mathrm{cm}^{2}\right.$ ) aluminized Mylar ${ }^{*}$, which was glued in place over the window gap. The gas in the detector is isobutane, which is flowed through the active volume at about 400 torr from a quartz tube. This pressure provides an optimal combination of high efficiency for betas and low cosmic-ray background. A schematic of the detector gas handling system is shown in Fig. 4.18. An automatic servo valve is used to keep the variations in the pressure of the gas flowing through the detector down to about 1 torr.

To perform tests of the spectrometer resolution, the pumping restriction can be isolated from the spectrometer by two gate valves and the collimator removed to allow the insertion of an electron gun. The electron gun used for these tests is a photoemissive source which can be biased to a high potential. Since most of the electric field gradient 


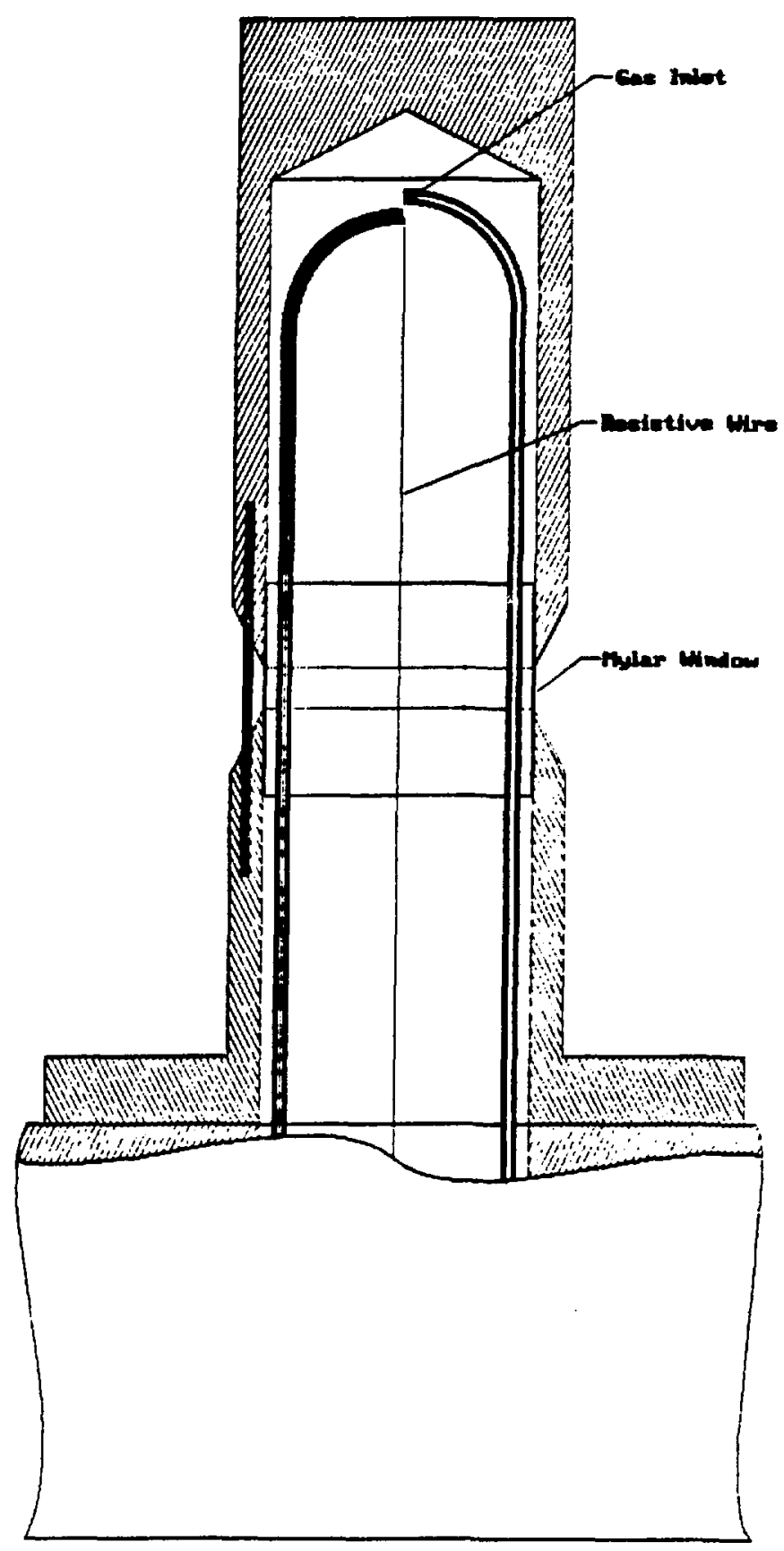

Fig. 4.17. The active portion of the detector. 


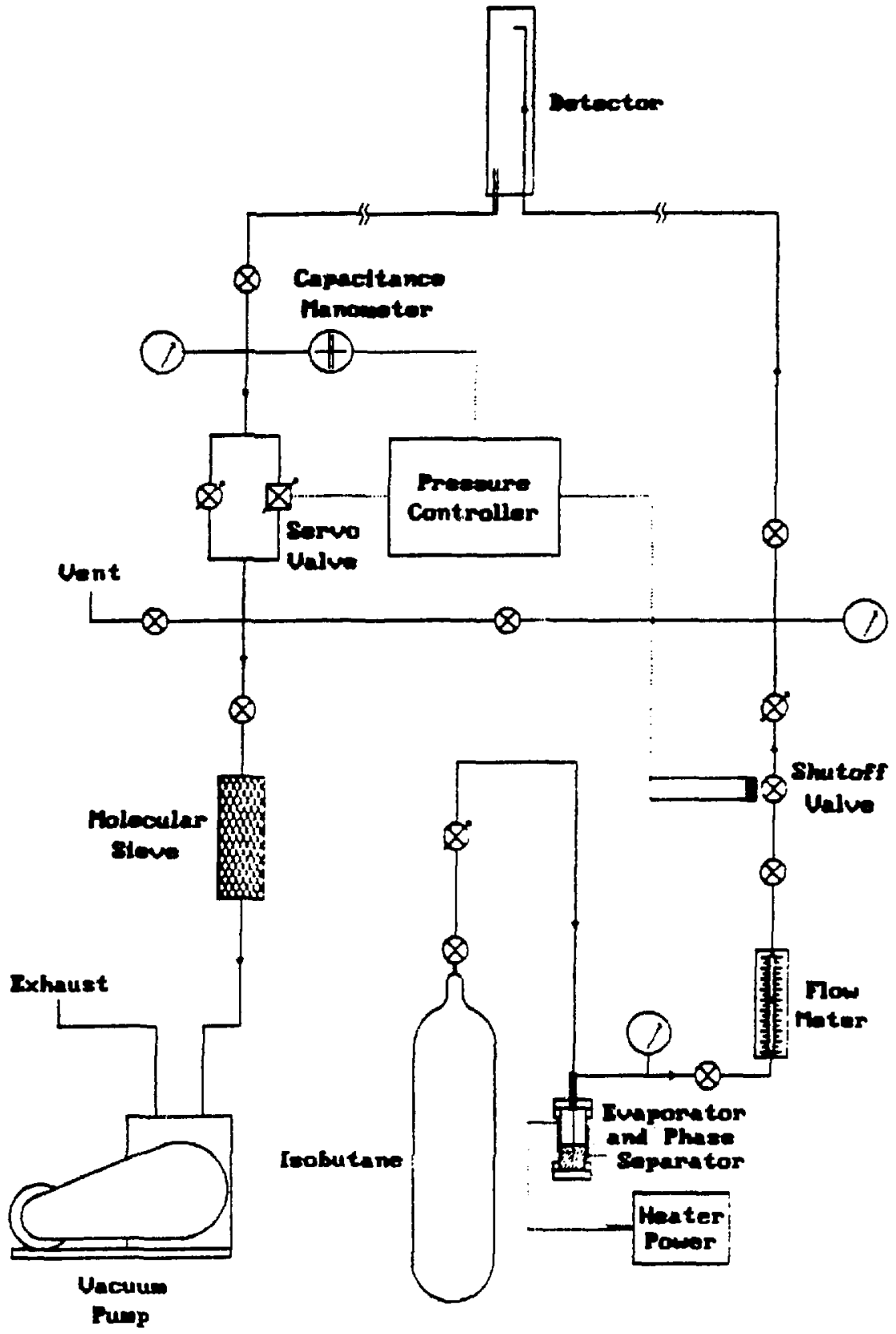

Fig. 4.18. The detector gas handling system. 
is near the surface of the source, the gun is a virtual point source of electrons.

\section{Data Acquisition System}

The signals from the detector and the beta monitor are processed by the data acquisition system, shown in Fig. 4.19. The signals from each end of the resistive wire in the detector are preamplified separately, summed, and amplified. The resulting signals are digitized and read by an IBM PC AT computer through a CAMAC bus. The computer performs the division to determine the position of the signal on the wire, which is given by:

$$
x=v_{1} /\left(v_{1}+v_{2}\right)
$$

where $v_{1}$ and $v_{2}$ are the amplitudes of the signal from each end. The signals are also gated by the computer; that is, separate spectra of amplitude gated by position and position gated by amplitude are saved.

The amplified signals from the beta monitor are transmitted from the beta monitor NIM bin, which is at the same potential as the source tube, to ground potential by means of a fiber optic link. They are then analyzed by an multichannel analyzer [LECO1], and the spectrum from the MCA is downloaded to the computer at the end of each run. A linear gate stretcher is used as a high-quality lower level discriminator, since the discriminator in the MCA was not adequate for the purpose. The gain of the beta monitor system was monitored by a pulser.

The high voltage on the source tube and the beta monitor electronics is controlled through a programmable $0-10 \mathrm{~V}$ DC voltage 


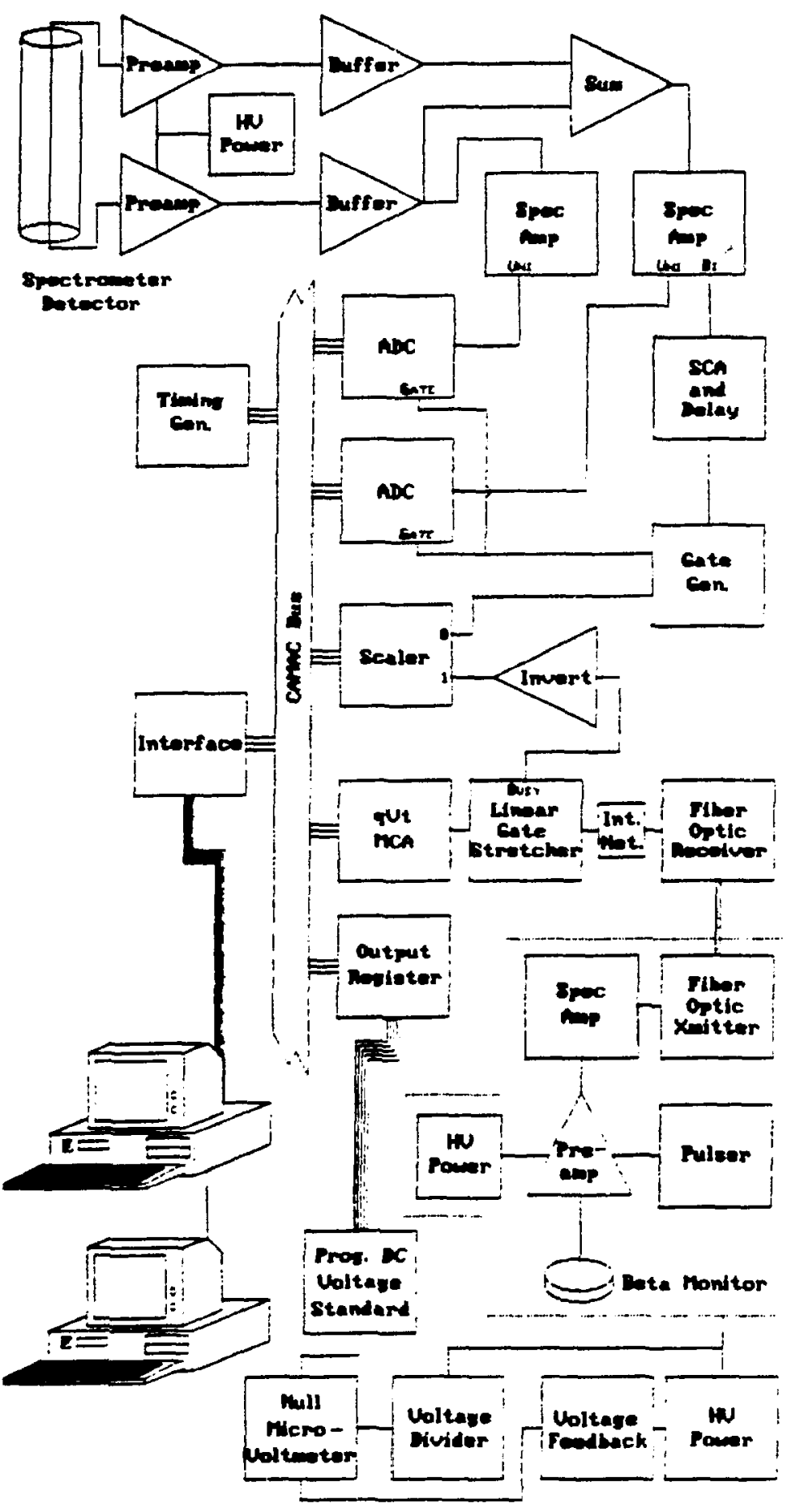

Fig. 4.19. The daca acquistion system. 
standard accurate to 6 significant digits [EDC01]. It is programmed by the same computer that acquires the data. The voltage output from the high voltage power supply is divided by a 5000 to 1 calibrated resisitive divider and compared to the reference voltage from the voltage standard. The result is then fed back into the power supply. This system provides an accuracy of better than 1 volt, with minimal variation.

\section{Calculated and Measured Characteristics of the Apparatus}

As the apparatus was assembled, its characteristics were compared to expected values to ensure that it would perform adequately. A summary of these expectations and tests follows.

\section{Pumping Restriction}

The first parts of the apparatus constructed were the cryopumps for the pumping restriction. Speed tests on both were performed using an apparatus similar to that shown in Fig. 4.20. A known volume is filled with gas and this gas is then leaked into the chamber containing the punp. The decrease in pressure per second $(\Delta P / \Delta t)$ in the known volume gives the gas flow:

$$
\mathrm{V}(\Delta \mathrm{P} / \Delta \mathrm{t})=\text { Flow in Torr-1iters / second. }
$$

Dividing this number by the pressure in the chamber containing the pump gives the speed of the pump in liters / second. The test of pumping speed for the cryopump in the center of the pumping restriction was performed with the restriction tubes in place; however, since the 


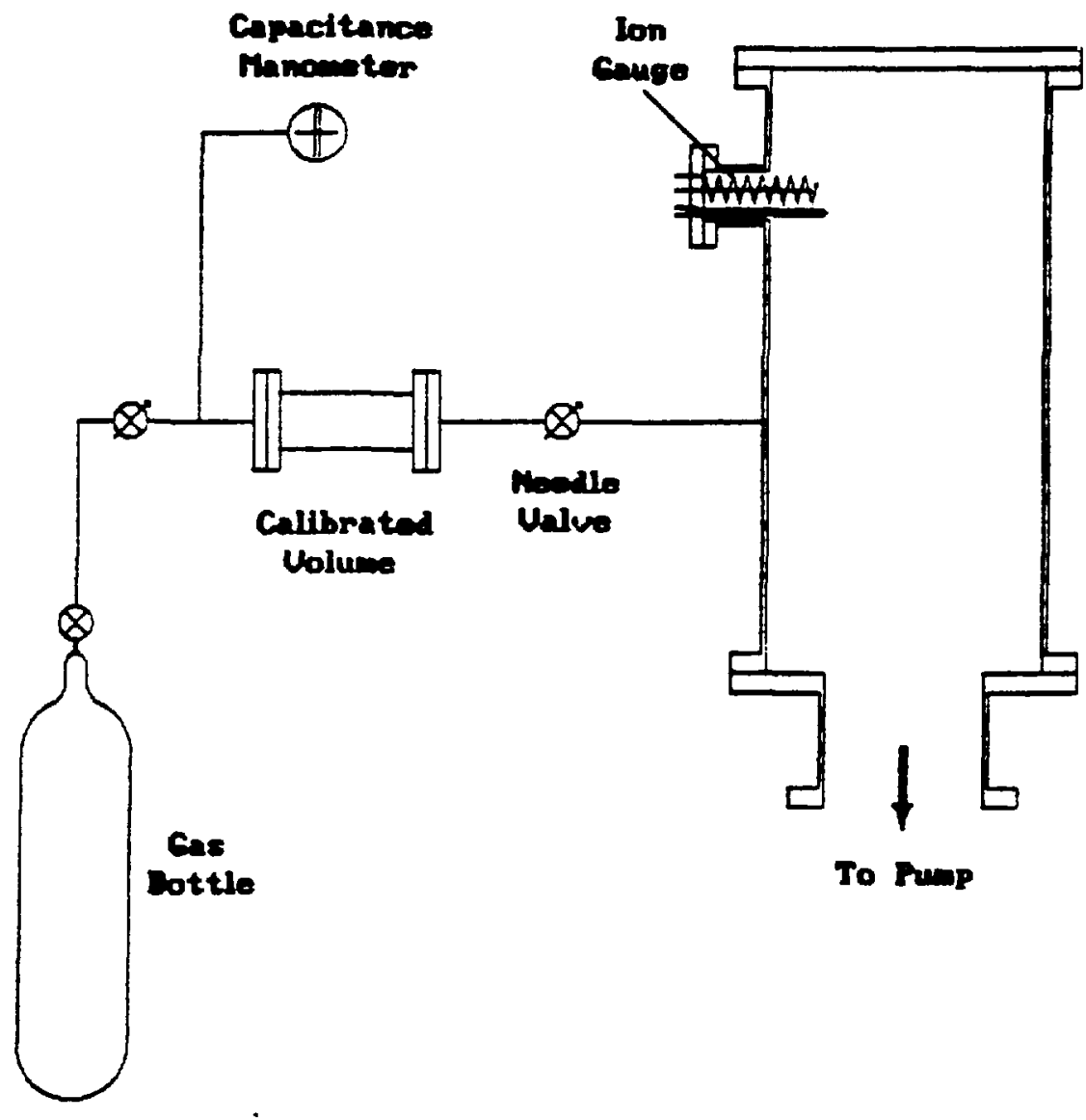

Fig. 4.20. Apparatus used to measure pumping speeds. 
pressure in the vacuum vessel was measured at the opposite end of the restriction from the gas inlet, there was no gas flow between it and the cryopump, so at equilibrium the pressure measured was the same as the pressure at the cryopump.

The pumping speed for the chamber cryopump was expected to be limited by the conductance to the pumping surface. This conductance was calculated to be about $3500 \mathrm{l} / \mathrm{s}$ for tritium. Since tritium is so difficult to handle, hydrogen and deuterium were used in the speed tests. The measured speed for hydrogen was $5700 \mathrm{l} / \mathrm{s}$ and that for deuterium was $4400 \mathrm{l} / \mathrm{s}$. These values confirm that the limiting factor in the pumping speed is the conductance, since it should vary as $\mathrm{M}^{-1 / 2}$, where $M$ is the molecular weight. The deuterium value is slightly higher than would be predicted this way, indicating that there is also some component of the speed that depends or the speed of the pumping surface. Therefore, the expected pumping speed for tritium is at least $3300 \mathrm{l} / \mathrm{s}$, but probably somewhat higher. After pumping $2200 \mathrm{~T}-\ell$ (about 3 standard litors) of deuterium, the pump showed no sign of any deterioration in 5 : The total load of tritium in the pump is small enough, even when the entire inventory of tritium ( 1 standard liter) is in it, that the heat generated by the radioactivity should not affect the pumping speed or the ultimate vacuum.

Similar tests were performed on the center cryopump. Its hydrogen pumping speed was measured to be about $6300 \ell / \mathrm{s}$, and deuterium gave a speed of $5400 \mathrm{l} / \mathrm{s}$, indicating a speed for tritium of at least $3700 \mathrm{l} / \mathrm{s}$, but probably about $4000 \ell / \mathrm{s}$. The pump was filled with 2 standard liters 
of hydrogen and deuterium and no degradation in pumping speed was observed.

Based on these observations, some estimate of the contamination of the spectrometer by tritium from the source can be made. At a pressure in the acceleration gap of $1 \times 10^{-5}$ torr, the partial pressure of tritium expected in the cryopump tank is about $7 \times 10^{-11}$ torr. Through the $1 \mathrm{~cm}$ diameter collimator, which has a conductance of $20 \mathrm{l} / \mathrm{s}$, this implies a contamination rate of $1.4 \times 10^{-9} \mathrm{~T}-\ell / \mathrm{s}$, or $5 \times 10^{10}$ molecules/sec. This amounts to $400 \mu \mathrm{Ci}$ per day.

\section{Extraction}

When the pumping restriction construction was completed, the extraction efficiency was measured using the apparatus shown in Fig. 4.21. First a ${ }^{169} \mathrm{Yb}$ source was placed in a vacuun vessel about $2 \mathrm{~cm}$ from a $100 \mathrm{~mm}^{2}$ solid-state surface barrier detector, and the spectrum was recorded, as shown in Fig. 4.22a. Then the source was placed at one end of the pumping restriction and the detector was placed at the other, at the location of the collimator. Since the detector had the same area as the collimator, the efficiency of extraction of betas to the collimator could be determined from the count rate in the detector. The spectrum of extracted betas is shown in Fig. 4.22b. The extraction efficiency for each peak was determined by subtracting the extracted spectrum multiplied by a constant from the calibration spectrum until the peak in question disappeared. This automatically corrects for the scattered electrons which make up most of the background under the lowenergy peaks. The efficiency clearly varies with energy, which is an 


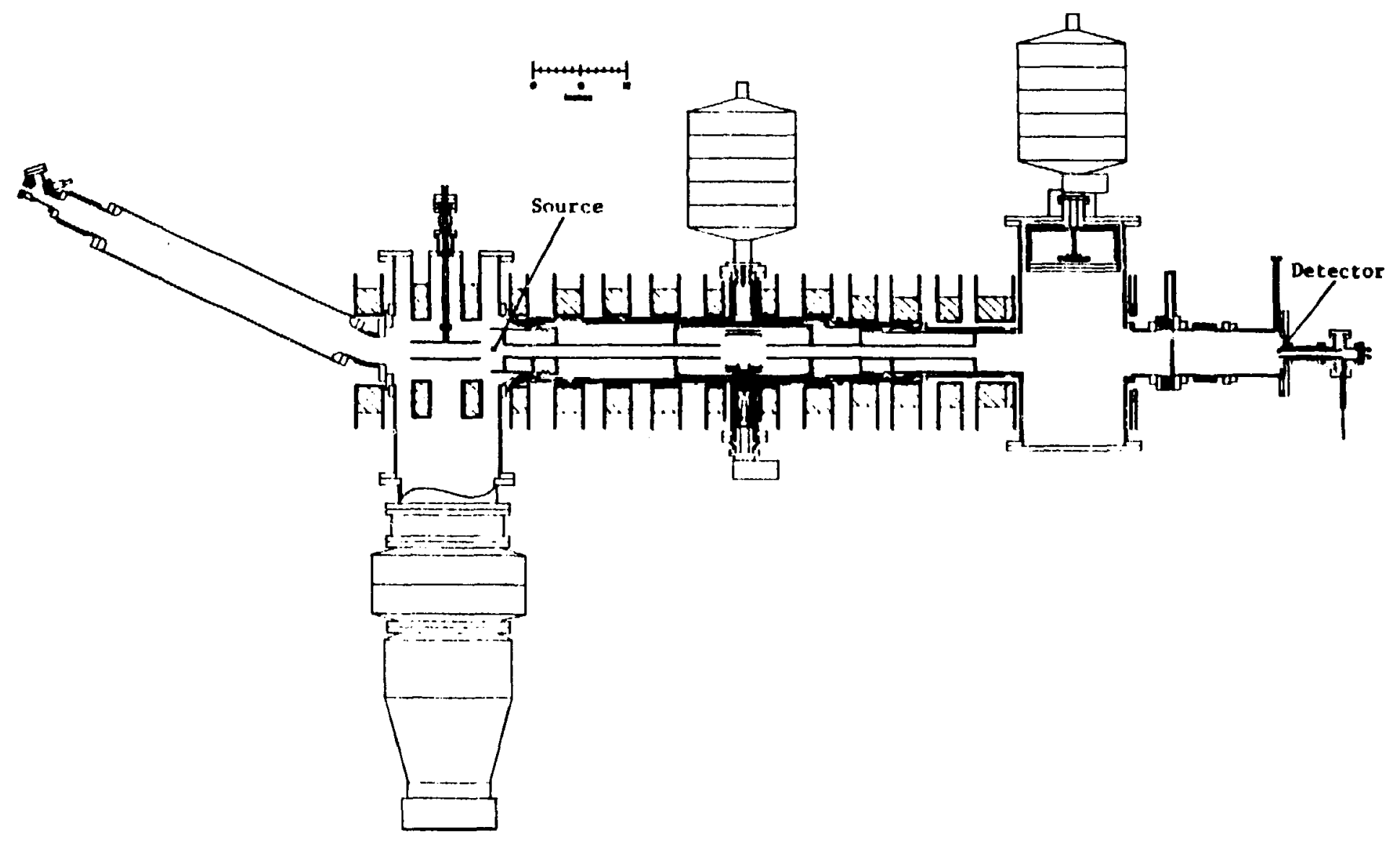

Fig. 4.21. Apparatus used to determine extraction efficiency. 


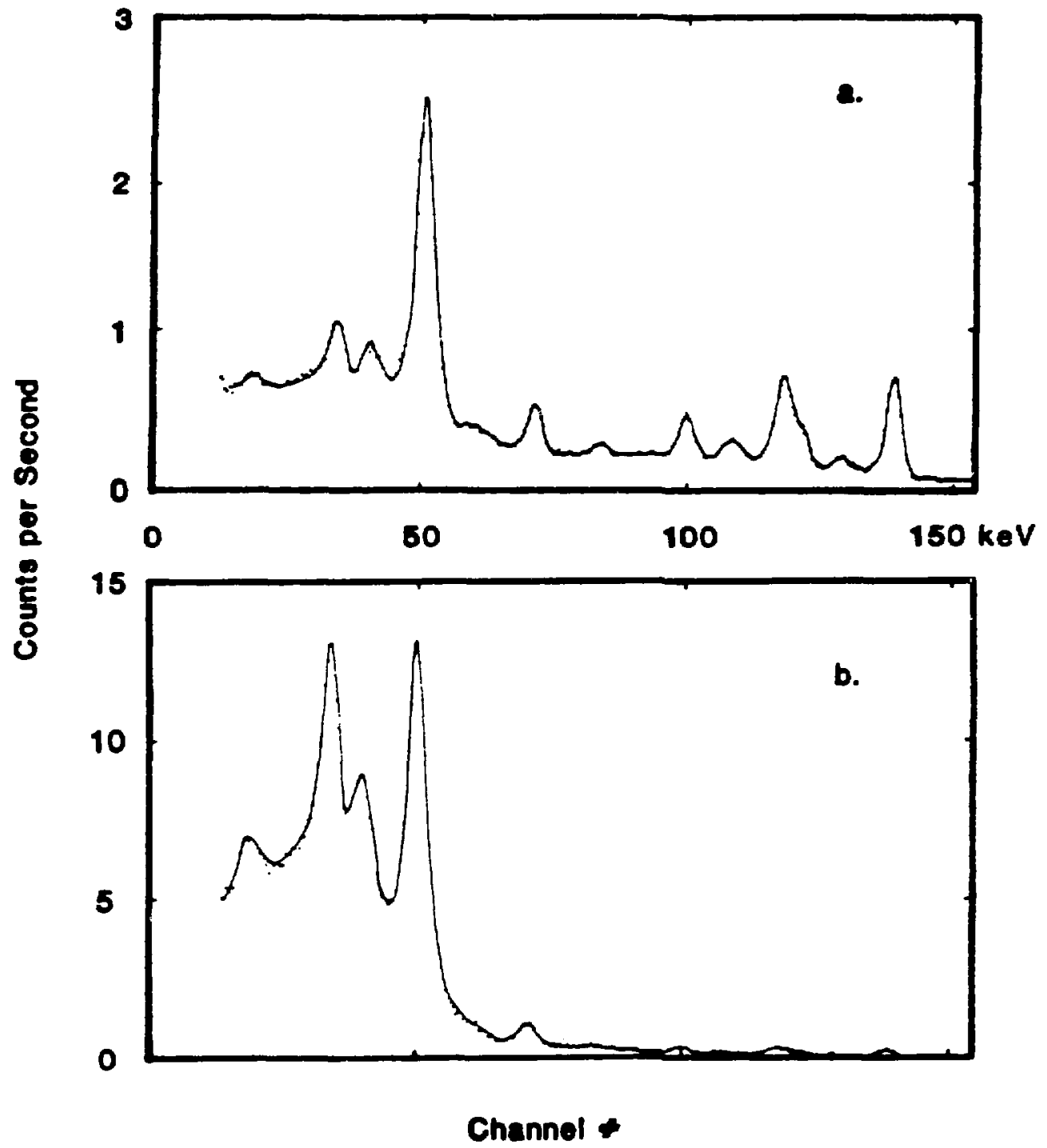

F1g. 4.22. Extraction efficiency test data. a) Spectrum recorded in the detector with source $30 \mathrm{~mm}$ away. b) Spectrum recorded in detector with source and detsctor as shown in Fig. 4.21. 
expected effect. The extraction efficiency for the $34 \mathrm{keV}$ peak was determined to be $25 \%$ using this method, almost exactly the efficiency predicted.

\section{Molecular Source}

The source region has, for the most part, lived up to expectations. It has never been run in atomic mode, however, since the diagnostics for the atomic mode have not yet been completed. The temperature of the source tube has proven very easy to control by varying the $\mathrm{LN}_{2}$ flow through the thermal transfer tubes. Two separate chambers were built into each copper clamp ring, for redundancy. With both chambers operating, the tube can be cooled to about $100 \mathrm{~K}$. One of the chambers developed a leak, however, so it was not used during most of the data runs. With only one chamber operating, the tube can only be cooled to about $115 \mathrm{~K}$, which is certainly adequate. A more serious concern was that the tube would generate such a severe discharge that it would be unable to hold high voltage. It was anticipated that a discharge could not be maintained across magnetic field lines, but only along them, so the area of most concern was the acceleration gap. Using argon gas at a pressure of about 10 microns, high voltage was applied to the tube. The discharges could be seen with the naked eye. Initially, there was a significant discharge in the tank which contains the acceleration gap, but it was found to be due to an inadvertent reversal of the field of the solenoid, which caused the field in the tank to go to zero. When this problem was remedied, no further discharge was observed at the acceleration gap. However, a significant discharge did 
occur along the surface of one of the sapphire thermal links to the tube, probably the result of a defect in the piece. The current in this discharge depended on the ambient pressure and on the temperature of the cathode (in this case the tube). The discharge was found to be small enough that tritium could be run through the system at an adequate pressure to obtain data, albeit not the pressure originally anticipated. The main effect of this discharge is a slight heating of the source tube. Over time, the problem has slowly improved.

The leakage of tritium through the knife-edge seals between the dissociator and the source tube is monitored by an ion gauge mounted nearby. The pumping speed away from this region is quite low, so the pressure is very sensitive to small leaks in tiese seals. The leakage appears to be minimal, although it is difficult to estimate the actual leakage from the pressure. Using a measurement of the flow rate and the pressure of the gas at the ends of the source tube, however, it was possible to determine that the flow was consistent with being almost completely through the source tube.

The contamination of the tritium gas by hydrogen during data runs is an important parameter to monitor, even though the number is not required for the analysis of the data, except for a minor correction for energy loss in the source. Initially, much of the hydrogen and deuterium in the system were in the palladium leak in the gas handling system, which had adsorbed considerable quantities of those gases into its surfaces. Inevitably, also, the surfaces of all the vacuum vessels contained quite a lot of hydrogen. It was expected that the first tritium which was run through the system would be depleted rather 
quickly, but the exact rate was not known. In practice, this problem has not been as severe as expected. After the first run, the hydrogen contamination increased during the runs from almost zero to about 128 , and then leveled off, apparently reaching some kind of equilibrium. Even after very long runs earlier in the experiment, the hydrogen and deuterium contamination never exceeded 158 .

\section{Detector}

The detector was tested for gain, position and energy resolution, and background. During development, the detector was tested with a ${ }^{55} \mathrm{Fe}$ gamma ray source $\left(E_{\gamma}=5.6 \mathrm{keV}\right)$ and an electron gun. After these tests determined the best gas, gas pressure, and window material, a ${ }^{103_{R u}}$ source was used to test the detector with real electrons from a source. One problem which was encountered in building a working detector was the window material. Originally, Kapton was the material of choice, since it stretches very little and is extremely strong. However, it proved impossible to get Kapton which was thin enough to let $26 \mathrm{keV}$ electrons through without completely destroying the energy resolution through straggling. The thin Kapton films were also plagued by pinholes, which meant that the detector leaked severe1y. Mylar ${ }^{\circledR}$, which is much stretchier, was then tried, and was found to be adequately strong to hold a pressure differential of an atmosphere in the desired geometry, if more support was added. It can be obtained in thinner films than Kapton, and the material used was free of pinholes. The other major problem proved to be the detector gas. Many combinations of gases were tried, but pure isobutane proved to be most effective. For these low- 
energy electrons, the detector gas must be as dense as possible to provide adequate stopping power while having a low average $z$ to prevent backscattering. Of critical importance in the choice of detector gas is the lack of tritium or other radioactive isotopes in the gas itself, since these will cause a background which cannot be avoided. The isobutane which was used for these measurements was $99.5 \%$ pure, obtained from deep wells free of tritium and ${ }^{14} \mathrm{C}$ contamination. The major disadvantage of isobutane is its tendency to build up deposits on the detector wire, requiring periodic regeneration. This layer of material results from polymerization of the gas induced by the passage of the charged particles and the high voltage on the wire. The regeneration is performed by heating the wire in oxygen (usually just room air at one atmosphere) for several minutes.

The gain and background rate of the detector depend on the pressure of the gas in it. The gain goes up with decreasing pressure (as long as the pressure is comparable to an atmosphere), and the background, which is primarily from cosmic ray interactions and from radioactivity in the detector gas itself, goes down. Therefore, the optimal pressure is the lowest possible which still has a high detection efficiency. The gain was found to depend almost exclusively on the detector pressure, so the pressure was held constant to an accuracy of 1 Torr, which resulted in a gain stable to within about 48. The gain and resolution of the detector did not vary with count rate. In the case of the measurements reported here, the detector pressure was kept at about 400 Torr. At this pressure, the background rate (gated for position and energy) is about .004 counts/sec, mostly from cosmic rays. 
For $26 \mathrm{keV}$ electrons, the energy resolution in the detector is 208 and the position resolution is $4.0 \mathrm{~mm}$. Typical position and energy spectra from the detector are shown in Fig. 4.23 .

\section{Spectrometer}

The spectrometer resolution has proven to be the most recalcitrant parameter of the experiment to bring into agreement, with expectations. Initial measurements were attempted using a $103_{\mathrm{Ru}}$ source. The resolution was inadequate to resolve the $\mathrm{L}_{2}$ and $\mathrm{L}_{3}$ peaks expected at 36.60 and $36.75 \mathrm{keV}$. Source problems were suspected, so an electron gun was constructed, as described earlier. The first spectra obtained from this diagnostic showed double peaking (Fig. 4.24a). Changing the horizontal component of the magnetic shielding eliminated this characteristic, and eventually a resolution of $25 \mathrm{eV}$ at $25 \mathrm{keV}$ electron energy was obtained (Fig. 4.24b). This width includes the width of the detector window, which acts like a square function $21 \mathrm{eV}$ wide at $25 \mathrm{keV}$. Removing this width from the resolution results in an effective width of $21 \mathrm{eV}$ FWHM.

The ideal measurements of spectrometer resolution depend on having a gaseous source which behaves like the tritium that is actually used. The isotope $83 \mathrm{~m}_{\mathrm{Kr}}$ is used for these measurements. In addition to being a noble gas, it has a short ( $1.8 \mathrm{hr}$ ) halflife and can be produced from a long-lived parent, ${ }^{83} \mathrm{Rb}$, which has a halflife of 86 days. A number of $83 \mathrm{~m}_{\mathrm{Kr}}$ sources were prepared, all using emanation of the krypton from a $83_{\mathrm{Rb}}$ source. The initial source consisted of the rubidium implanted in a resin. No sign of krypton was seen using this source. It was then 
a)

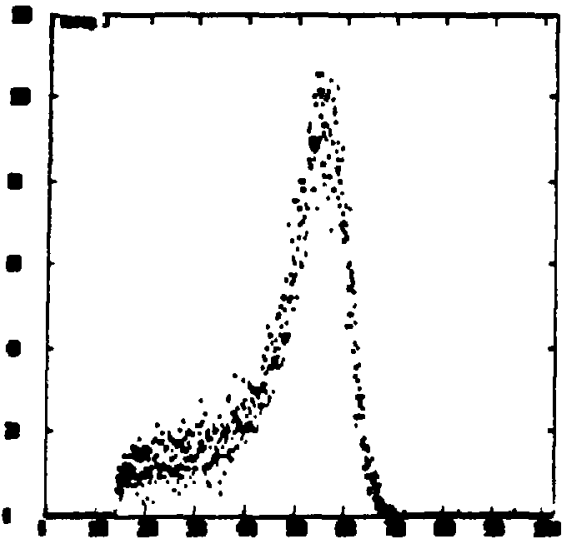

c)

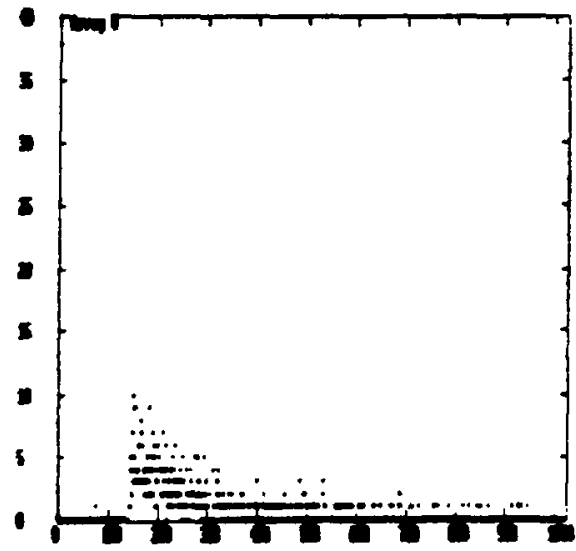

e)

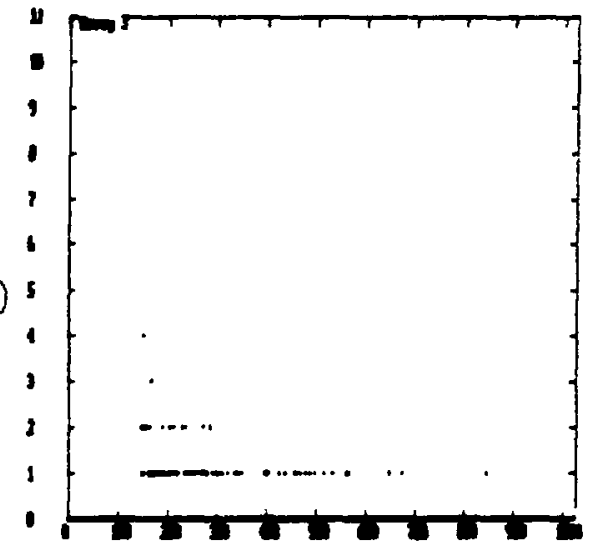

b)

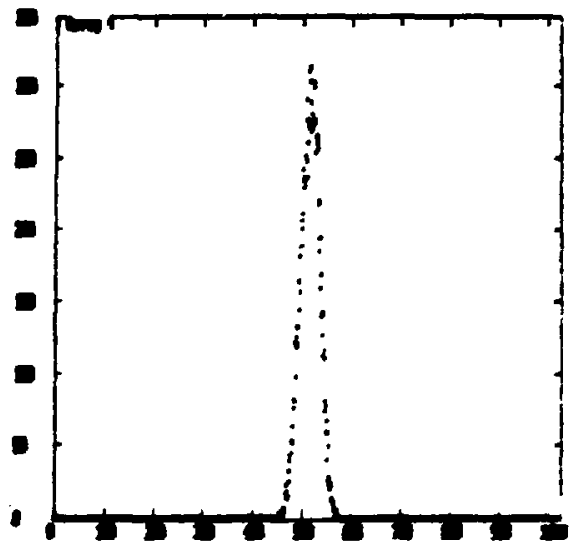

d)
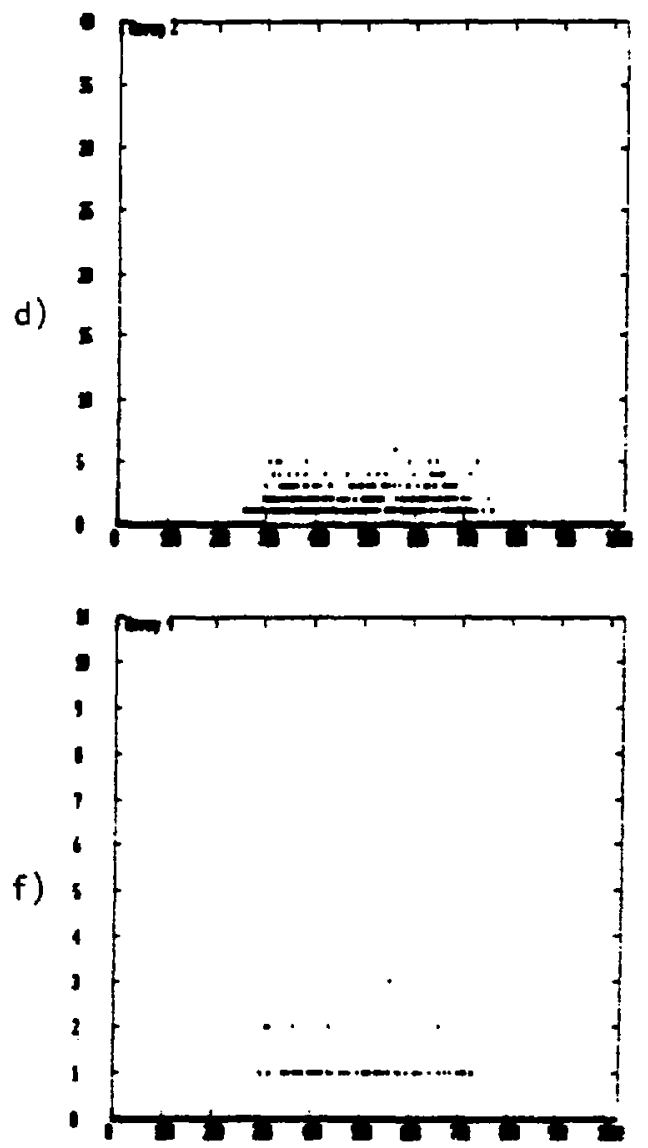

18. 4.23. Typical position and energy spectra recorded in spectrometer detector. Tritium data was taken for $600 \mathrm{sec;}$ background for $3000 \mathrm{sec}$. a) Energy spectrum for tritium, gated by position. b) Position spectrum for tritium, gated by energy. c) Raw background energy spectrum. d) Raw background position spectrum. e) Background energy spectrum gated by position. The background is determined by the sum between channels 430 and 690 of this spectrum. f) Background position spectrum gated by energy. 
suggested by $K$. Wolfsberg [Wo185] that perhaps the krypton was not emanating from the resin. He prepared a new source, somewhat stronger, with the $\mathrm{Rb}$ in a mixed $\mathrm{Na}-\mathrm{Rb}$ stearate. The krypton conversion electrons were indeed seen from this source. The initial measurements, taken after a data run in October 1985, showed a FWHM of about $65 \mathrm{eV}$ (Fig. 4.25). Field mapping around the spectrometer after this measurement revealed two problems: first, several conductor bars in the spectrometer had fallen out of position and created dipole fields in the horizontal direction, and secondly, a gradient of $80 \mathrm{mG}$ from the field in the room was found in the horizontal component of the residual field going from top to bottom. The conductors in the spectrometer were repaired and a coil was added to the magnetic shielding to compensate for the gradient. Resolution measurements using the electron gun were again made, and the double peaking behavior which had been observed at zero field disappeared. It had been a result of the gradient in the horizontal field, which had gone undetected because of inadequate accuracy of the magnetometer probes used during the initial measurements. A new threeaxis magnetometer was purchased in the interim, making much more precise measurements possible.

After the improvements, the resolution was measured with the electron gun as a function of detector position, source position, and electron energy. The measurements made at three energies, $18 \mathrm{keV}$, $26 \mathrm{keV}$, and $34 \mathrm{keV}$, allowed a determination of the relative contributions to the resolution width of field errors (errors, for example, in the magnetic shielding) and conductor position errors. The former varies as the energy, while the latter varies as the square root 

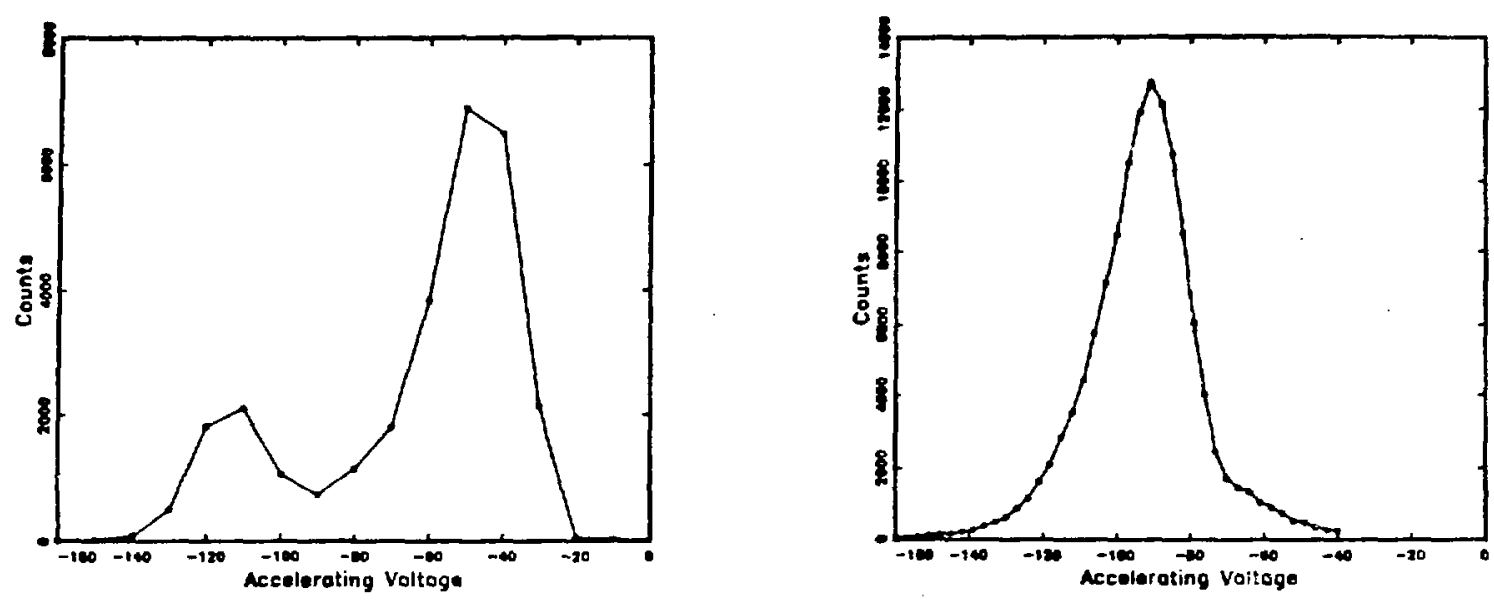

Fig. 4.24. Resolution functions obtained with electron gun, June 1985: a) with fields nominally zero. b) with optimal offset in horizontal compensation field.

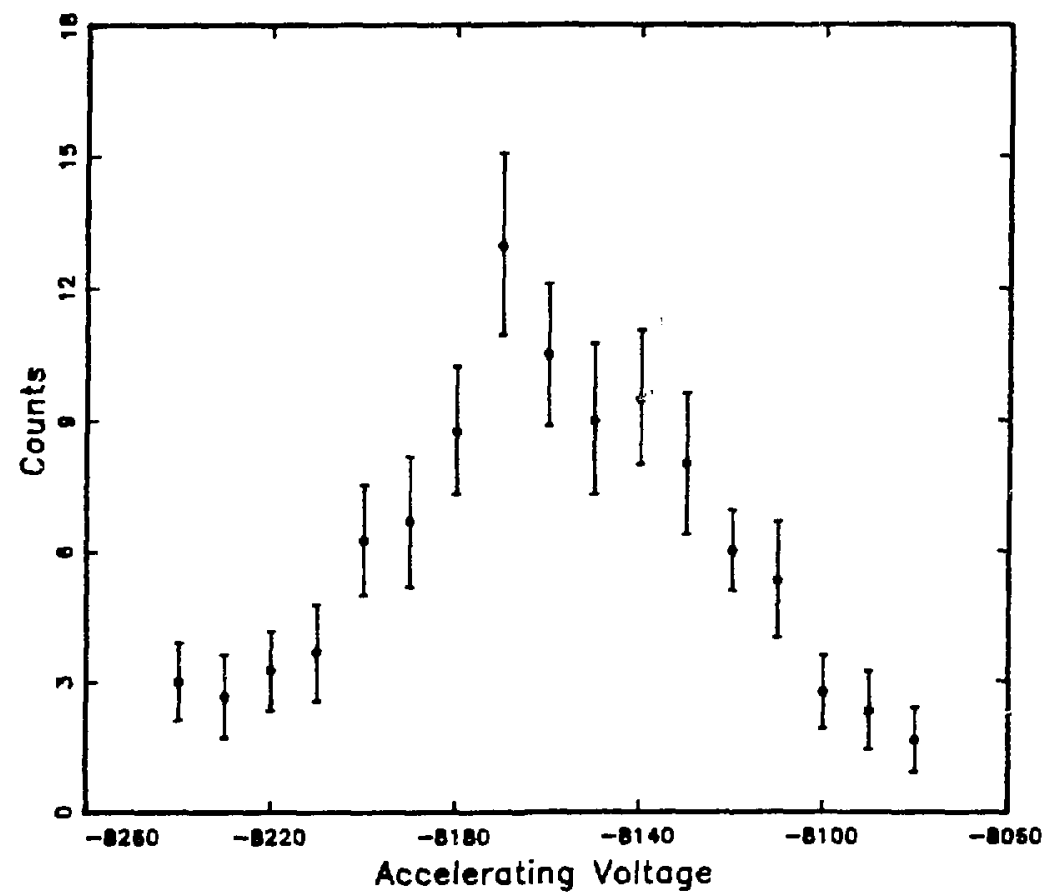

Fig. 4.25. First Krypton resolution function, obtained in December 1985. 
of the energy. The results of these measurements allowed the resolution width due to field errors to be minimized. The resolution obtained at $26 \mathrm{keV}$ is shown in Fig. 4.26.

The variation of the resolution with detector position was also measured. The detector was moved forward and backward along the axis of the spectrometer, and the shape of the response function was measured. The results, seen in Fig. 4.27, show that the symmetry of the peak depends on the position of the detector. The position was then optimized before the data was taken. This procedure also allowed a measurement of the dispersion of the spectrometer, which was found to be $10.5 \mathrm{eV} / \mathrm{mm}$.

After the January 1986 data were taken, tests were performed with the krypton source. In this case, the energy of the line could not be changed, so the acceptance energy of the spectrometer was varied to obtain resolution functions at 22,26 , and $34 \mathrm{keV}$. The variation of the width with energy strongly suggested that the dominant contribution was that of the field errors, probably from the residual fields from the solenoid extraction region. These fields diverge away from the focus coil, and change sign as the residual field from the solenoid overwhelms that from the focus coil. In addition, the spectrometer was constructed with an odd number of electron crossings of the axis, which means that the effects of the residual fields cannot cancel as well as they would for an even number. A compensation coil was constructed to nearly eliminate the axial field in the spectrometer volume. It allowed the spectrometer resolution to improve to about $45 \mathrm{eV}$. However, another error in the field was detected, this time a residual field from the 


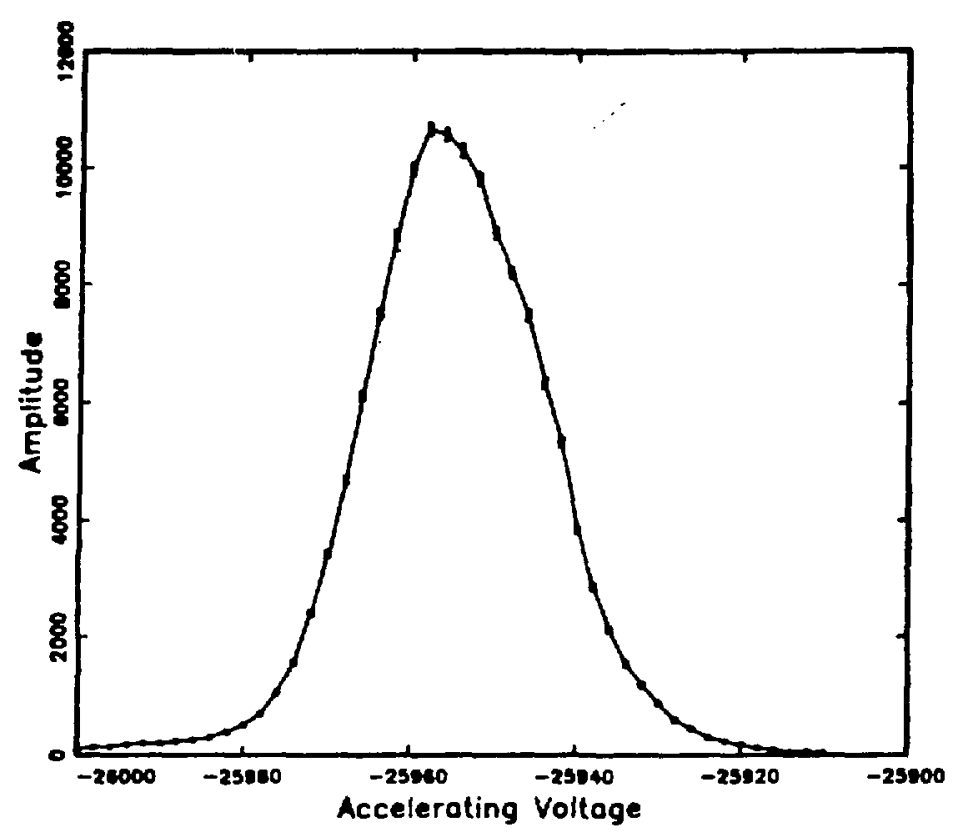

Fig. 4.26. Optimal electrun gun rese tion obtained in February 1986.
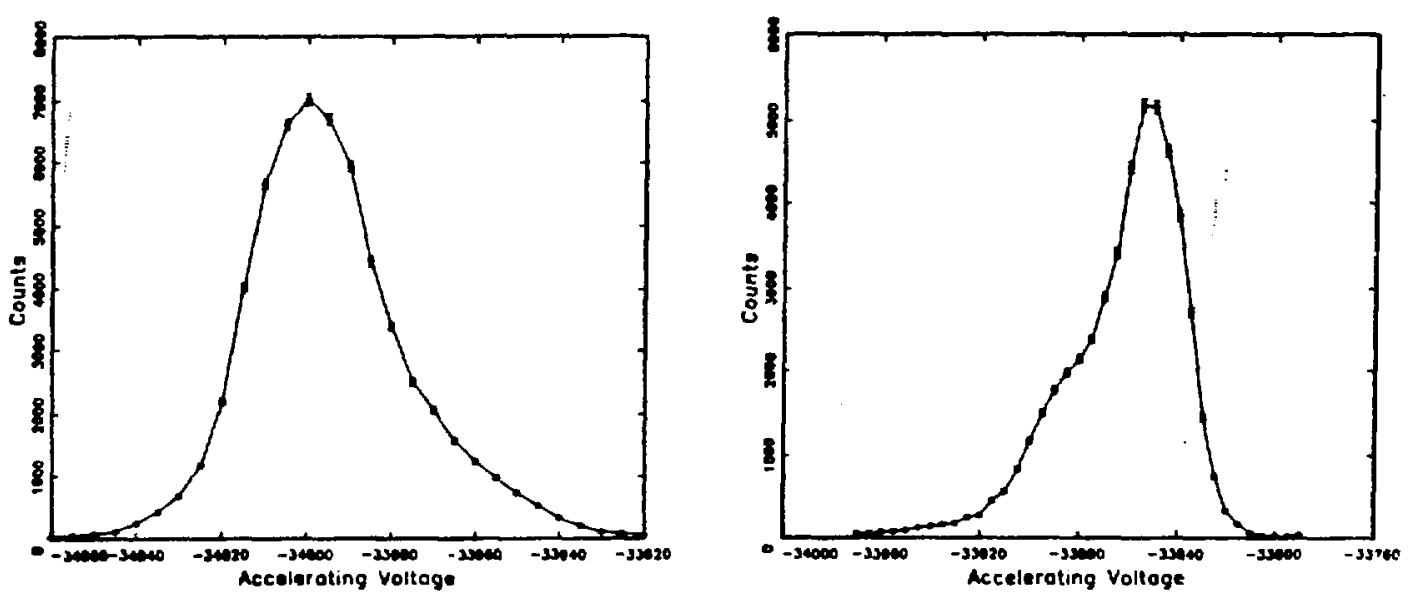

Fig. 4.27. Resolution function at different detector positions. a) $z$ - optimal position $+5.1 \mathrm{~mm}$. b) $z$ - optimal position - $5.1 \mathrm{~mm}$. 
spectrometer itself, probably due to an element in the spectrometer which had moved. Rather than open the spectrometer, the error was compensated as well as possible by introducing an offset into the magnetic shielding. This allowed the spectrometer resolution to reach $37 \mathrm{eV}$.

Finally, the spectrometer was tested to see if tritium decaying on the walls of the source tube could be detected in the spectrometer detector. Initial measurements indicated that, with no gas in the tube, about 38 of the count rate remained. However, this effect turned out to be a result of a reversed field in the solenoid, which allowed some betas from the walls of the source tube to enter the spectrometer. When the fields were corrected, no sign of the betas from tritium on the walls was observed, and none has been observed since.

\section{Beta Monitor}

The performance of the beta monitor is crucial for obtaining good data. The beta monitor was positioned by moving it into the electron beam until the effect of its presence began to affect the spectrometer counting rate. It was positioned just outside of that radius. Tests of the counting rate with no tritium gas in the source and the magnetic fields all on indicated that no electrons originating from tritium on the walls of the source tube could be detected by the beta monitor. This result was expected because the electrons originating from the walls of the source tube cannot even traverse the pumping restriction tubes. It is possible to observe these electrons by making the field in the source lower than in the pumping restriction, in which case the 
electrons are not rejected by the pumping restriction tubes. When this was done, the beta monitor definitely registered the presence of these electrons, in numbers comparable to the number seen from the gas in the source tube. However, with the fields in their nominal configuration, the counting rate returned to the background level. No evidence of any betas from the walls of the source tube entering the beta monitor (or the spectrometer) has yet been observed with the fields in the correct configuration.

There were three problems encountered in getting the beta monitor to work as expected. Initially, it had been planned to accelerate the betas into the detector to raise the endpoint of the electrons from the source above the endpoint of the background from tritium on the surface of the detector. This did not work, however, because the detector was flooded by low energy electrons originating in the source tube and accelerated by the source potential. The beta monitor and its electronics are therefore floated at the same potential. as the source tube, so that an electron which originates in the source with some energy will deposit the same energy into the detector. An electronic threshold of about $13 \mathrm{keV}$ is set on the spectrum, well above the detector noise. This approach has the additional advantage of reducing background by rejecting those betas originating on the walls of the apparatus outside the source. Since the decelerating voltage experienced by those electrons entering the beta monitor is always more than $5 \mathrm{kV}$, these electrons are not detected above the lower level discriminator. A typical spectrum from the beta monitor is shown in Fig. 4.28. The lowenergy counts are the end of the tritium spectrum, and the peak at 

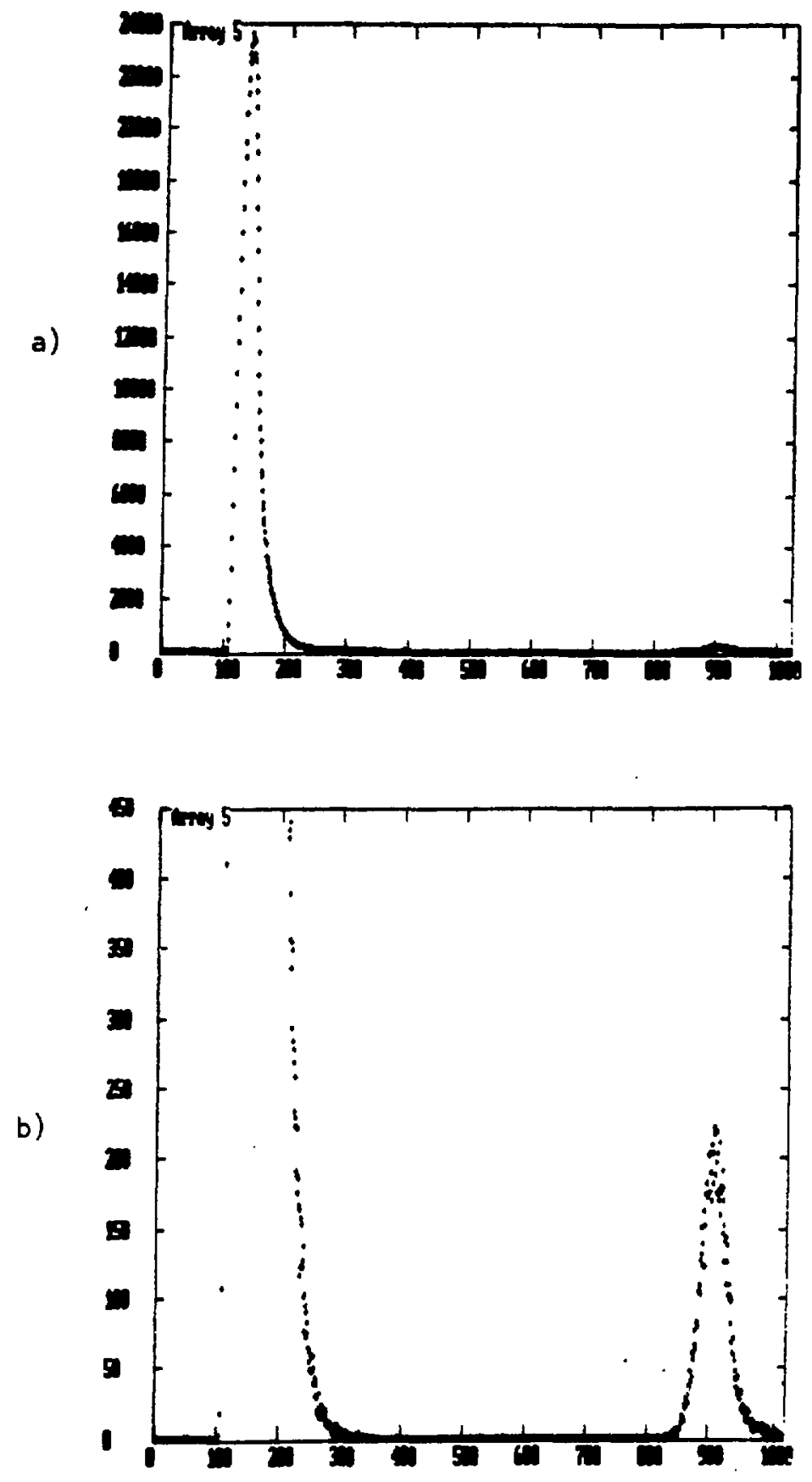

Fig. 4.28. Typical spectrum recorded from the beta monitor. Spectrum was taken for $200 \mathrm{sec}$. a) Full spectrum. b) Spectrum vertically expanded to show tritium endpoint and pulser peak. 
higher pulse height is that of a pulser that was used to monitor the gain of the electronics and to allow a deadtime correction.

The second problem was that of noise in the beta monitor electronics. Initially, a noisy preamp was replaced, which reduced the noise somewhat, but a persistent, intermittent noise remained. It was finally traced to a high-voltage spark which ignited the gas in a space heater located near the beta monitor. This problem was not solved until after the data sets presented here were taken, so the data reduction included a correction for the noise in those data sets. The noise was never of such a magnitude that it would have seriously compromised that data, but it did slightly increase the error in the determination of the actual source strength.

The final problem encountered with the beta monitor has been the buildup of background from tritium deposited on its surface. Ordinarily, with the detector under high vacuum, no change in this background is seen over long periods of time, even when tritiun is running normally. However, the electricity in the building has been shut off for periods of a week or more, so that the cryopumps were turned off and the entire source region filled with tritium to about $5 \times 10^{-3}$ Torr. After each of these episodes, the background in the beta monitor has been observed to increase. For the first data set presented here, the signal-to-background ratio in the beta monitor was about 3 , but it had decreased to 1 by the last data set taken in March 1986. This is not really a statistical problem, because the number of events typically seen during a $200 \mathrm{sec}$ run is about $1 \times 10^{6}$, but it presents deadtime problems and could eventually become a statistical problem. An 
interlock is being constructed so that the beta monitor can be removed from the vacuum chamber when the pressure of tritium is high, and a new detector has been obtained. 


\section{Chapter 5:}

\section{Data Acquisition, Reduction, and Analysis}

\section{Overview of the Acquisition and Reduction Procedure}

A beta spectrum is acquired by varying the voltage of the source tube, while maintaining a constant magnetic field in the spectrometer. The activity of the source is monitored by the beta monitor at the same time the data is taken. After every two data points are acquired, a short calibration run with the source at the highest possible potential is taken to monitor any long-term variations in experimental parameters.

The count rate for each data point is obtained from a sum window in the spectrum of energy gated by position from the spectrometer detector. The points are normalized to the count rate in the beta monitor, so that pressure fluctuations in the source are removed from the data. These normalized data points are then input into the data analysis programs.

Overview of the Analysis Procedure

The data are fitted to a predicted spectrum, which includes all the significant effects in both the beta spectrum and the apparatus. These effects include the screening corrections, weak magnetism, a linear approximation for the effects of nuclear recoil, the effects of the experimental resolution and energy loss in the source, and a 
correction for the decays which occur in the acceleration gap, in which the potential differs from that of the rest of the source. The number of fitted parameters is variable. The errors in the fitted parameters, including the neutrino mass, are determined by varying the parameter in question and refitting the rest of the parameters. The errors in the parameters are also estimated using an approximation to the shape of the likelihood curve near the minimum, and from this the correlation coefficients between parameters can be determined:

Errors in the neutrino mass arising from systematic errors in nonfitted parameters (such as the resolution function) are determined by varying the parameter in question and observing the effect on the fit to the neutrino mass. The feffects of these errors on the actual data are compared to expected valhes derived from Monte Carlo simulations.

\section{Data Acquisition}

Data points werefacquired by varying the voltage of the source tube. The total energy of the electrons detected by the spectrometer detector is equal to the sum of the initial electron energy and the voltage on the source tube. Therefore, the energy of the electrons detected by the spectrometer is kept constant, which keeps the resolution of the spectrometer and the efficiency of the detector constant. In addition, the acceleration of the beta particles raises their energy well above the endpoint of any tritium decaying elsewhere in the apparatus, a property which dramatically reduces the background.

The voltage on the source tube was varied from 7000 to 9500 volts, and the spectrometer was set to a nominal electron energy of $26 \mathrm{keV}$, 
corresponding to initial electron energies from about 16500 to $19000 \mathrm{eV}$. The data points were taken in a random order to suppress any possible effects of drifts in experimental parameters. The data points were taken for $600 \mathrm{sec}$ each. After two points were taken, a calibration point was taken for $200 \mathrm{sec}$ with the voltage of the source tube at its maximum value of 9500 volts. During the data runs, the pressure of hydrogen isotopes in the dissociator was kept as constant as possible in order to keep the pressure in the source tube roughly constant. Pressures, voltages, currents, and temperatures throughout the apparatus were nonitored during the data taking.

\section{Data Reduction}

Each data point is derived from the position and energy spectra from the spectrometer detector taken at a specified source tube voltage. The energy spectrum gated by position is summed around the position of the peak. The gate on the position spectrum is set during the data acquisition. This spectrum has been observed to be extremely stable, since it is not sensitive to small gain shifts in the detector. The position and width of the "sum window" in the energy spectrum is varied according to the peak position, which is determined for each run by the positions of the peaks in the calibration runs before and after the data point, or by the data itself if the statistics permit. The error in the determination of the position of the peak is estimated at about 5 channels out of a typical peak position of 620 . The error in the sum introduced by this error is about. 58 , considerably less than the statistical error. Background is then subtracted so that the result can 
be corrected for source intensity by the beta monitor count rate. This implies that the background must be determined before the data is analyzed. The background rate is assumed to be flat under the beta spectrum and is measured by averaging the data taken at energies well above the endpoint. No energy variations or time variations (over a period of weeks) in the background rate have been observed.

The number of counts in the beta monitor is determined by summing the spectrum above the lower level discriminator, up to an energy significantly above the endpoint. The gain of the beta monitor and associated electronics is monitored by a pulser peak located well above the maximum energy of the beta spectrum. The pulser also allows a determination of the deadtime in the detector. During some of the runs, there was noise in the beta monitor electronics, whose contribution was estimated by measuring the number of counts in a region between the endpoint of the beta spectrum in the detector and the pulser peak.

Once all the corrections have been made, the beta monitor ratio $R_{\beta}$ is formed for the calibration runs, defined as the ratio of the number of counts observed in the spectrometer detector to the number of counts observed in the beta monitor during the calibration run. In order to minimize the statistical error associated with this ratio, the calibration voltage used is the highest voltage for which data was taken, corresponding to the lowest electron energy and therefore the highest count rate. This voltage was limited for the data presented here by leakage currents (see Chapt. 4). This ratio is used to search for systematis effects in the beta monitor which vary with time. If these ratios are nearly constant throughout the entire run, they can be 
averaged and the uncertainty introduced by individual measurements minimized. If there is variation, however, as was the case with the first run analyzed, then the ratio for each data point is determined frum the calibration runs immediately preceding and following it.

Since Poisson statistics apply to the total number of count at each point, the background must be added back in after the normalization. The normalized number of counts at a voltage $v$, then, is given by:

$$
y(V)=K\left(N_{D}-B K G D\right) / N_{\beta} R_{\beta}+B K G D,
$$

where $N_{D}$ is the number of counts in the energy window of the positiongated spectrum, $N_{\beta}$ is the deadtime-corrected number of counts in the beta monitor, and $\mathrm{R}_{\beta}$ is the ratio defined above. $\mathrm{K}$ is an arbitrary normalization constant which is set so that $\mathrm{KN}_{\beta} \mathrm{R}_{\beta}$ averages 1.0 at the endpoint, a condition which makes $y(V) \approx N_{D}$ near the endpoint. This condition is necessary for the maximum likelihood method of estimating the parameters, described later.

The error in $y(V)$ (hereafter denoted $y_{i}$ ) is determined by standard error propagation. If $A_{i}=K / N_{\beta} R_{\beta}$ and $\sigma_{A}$ is determined from the various components of $A_{i}$, then the error $\sigma_{i}$ is given by:

$$
\sigma_{i}^{2}=\left(N_{D}-B K G D\right)^{2} \sigma_{A}^{2}+N^{\prime} A_{i}^{2}+\left(1-A_{i}\right)^{2}\left(\sigma_{B K G D}\right)^{2}
$$

Where $N^{\prime}=N_{D}$ for $N_{D}>0$ and 1 for $N_{D}=0$. As explained below, this number is not actually considered to be the error, but is used to compare the relative size of the Gaussian error from $A_{i}$ to the error from the number of counts. At the endpoint, $A \approx 1.0$ and $\sigma_{A}<A$, so 
$\sigma_{i} \approx N_{D}^{1 / 2}$. This property allows the use of a maximum likelihood estimator.

Generation of Theoretical Spectra

An important part of the data analysis is a program, yockup, which generates theoretical beta spectra given input parameters. Spectra can be generated with various neutrino masses, backgrounds, endpoints, final states, efficiency functions, and resolution functions. A simulated spectrum, with variable statistics, can be generated and treated in every respect like actual data.

The spectrum is generated using the form given in Chapt. 2:

$$
\begin{aligned}
N(E)= & K F(Z, R, E) P_{e} E \sum_{i} \lambda_{i}\left(E_{0}-E_{i}-E\right)\left[\left(E_{0}-E_{i}-E\right)^{2}-m_{\nu}{ }^{2} c^{4}\right]^{1 / 2} \\
& \times\left[1+\alpha_{1}\left(E_{K}-E\right)+\alpha_{2}\left(E_{K}-E\right)^{2}\right] \Delta E,\left(E \leq E_{0}-E_{i}-m_{\nu} c^{2}\right)
\end{aligned}
$$

where $\lambda_{i}$ is the probability of the transition to final state $i, E_{i}$ is the excitation energy of final state $i$, and $\alpha_{1}$ and $\alpha_{2}$ are linear and quadratic efficiency function coefficients, in an expansion around $E_{K}$. $F(Z, R, E)$ is calculated using relativistic wavefunctions, and includes the screening corrections of Rose [Ros36]. Included in $\alpha_{1}$ are firstorder perturbation terms for the effects of weak magnetism and nuclear recoil, calculated by J. Friar [Fri85]. In the absence of other effects, these corrections give it a value of $2.312 \times 10^{-9} \mathrm{ev}^{-1}$, with $E_{K}=E_{0}$. A spectrum can also be generated that approximates an imaginary neutrino mass, which, if the true mass is zero, will statistically appear on occasion. This spectrum is generated by making the following approximation: 


$$
\varepsilon\left[\varepsilon^{2}-m_{\nu}{ }^{2} c^{4}\right]^{1 / 2} \approx \varepsilon^{2}-m_{\nu}{ }^{2} c^{4} / 2 \times \operatorname{sign}(\varepsilon)
$$

where $\varepsilon=E_{0}-E_{i}-E$. This approximation is the first-order expansion of the spectrum, and has the property of behaving reasonably above the endpoint.

The spectrum thus calculated may then be corrected for the effect of tritium which decays in the acceleration gap. Next, the spectrum is convoluted with a resolution function and with an energy loss spectrum (these two effects may be combined, if desired, into one resolution function). The resulting spectrum is then integrated over the width of the detector window (see Chapt. 4) at selected energies and has a background added to it. This result gives the expected number of counts at the voltage in the center of the window. To simulate real data, this number is used as the centroid for a Poisson random number generator [IMS01]. The resulting spectrum mocks up a spectrum in which the errors are purely statistical. To simulate a spectrum with errors in the beta monitor normalization, the points of this spectrum (minus the background) are used as the centroids of a Gaussian random number generator [IMS02], the width of which is the error in the beta monitor normalization. This spectrum, then, simulates actual data.

Mockup is used to estimate the statistics needed to obtain various limits on the neutrino mass. It can also be used to investigate the impact of various systematic effects, such as the effect of the resolution function and final states. Perhaps its most important function is as a check for the fitting algorithm. Various estimators 
were tried with simulated spectra, in order to determine the least biased parameter estimation technique.

\section{Fitting Data to the Theoretical Spectrum}

The data are fit to a theoretical spectrum generated as described above, using a Levenberg-Marquardt nonlinear maximum likelihood fitting algorithm [IMS03]. A set of programs that fit the data using various parameter estimators was written. In each case, theoretical spectra are generated using the algorithms of MockuP, with free parameters chosen from the following:

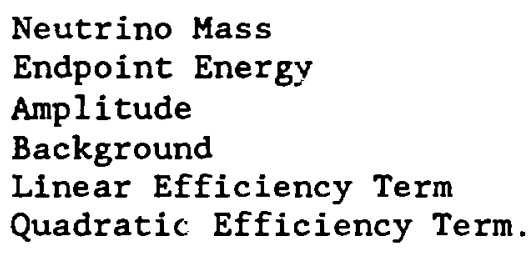

Any subset of these parameters may be fit, and those not fit are kept constant. This procedure allows the determinations of the error in each parameter, and also allows the fitting model to be varied somewhat.

An important problem was the determination of the optimal parameter estimator to use for the fits. The most familiar parameter estimator is $\chi^{2}$. The vector of parameter values $\xi$ is chosen so that:

$$
x^{2}-\sum_{i} \frac{\left[y_{i}-S\left(\xi, E_{i}\right)\right]^{2}}{\sigma_{i}^{2}} \quad \text { is a minimum, }
$$

where $y_{i}$ is the data point at energy $E_{i}, S\left(\xi, E_{i}\right)$ is the value of the theoretical spectrum at $E_{i}$ for $\xi$, and $\sigma_{i}$ is the experimental error in $y_{1}$. The expected minimum value of $x^{2}$, given properly determined 
experimental errors $\sigma_{i}$, is the number of degrees of freedom (the number of data points minus the dimension of $\xi) . \chi^{2}$ is a maximum likelihood estimator for data $y_{i}$ distributed as Gaussians about true (but unknown) values $m_{i}$. The likelihood of obtaining a vector of data $\Psi$ given a vector of true values $m$ is given by:

$$
L_{G}(\underline{m} ; y)-\Pi_{i}(2 \pi)^{-1 / 2} \exp \left[-\left(y_{i}-m_{i}\right)^{2} / 2 \sigma_{i}^{2}\right]
$$

This is not what we have in an experiment, however. Instead of $m$, we have $\underline{s}$, the values of $S\left(\xi, E_{i}\right)$. A useful theorem [Ead71] which can be used with any likelihood function is that, given a likelihood ratio $\lambda$ which is defined by:

$$
\lambda=\mathbf{L}(\underline{s} ; \underline{y}) / L(\underline{m} ; \underline{y}),
$$

then the "likelihood $\chi^{2}$, denoted by $\chi_{\lambda}{ }^{2}$, given by:

$$
x_{\lambda}^{2}=-2 \ln \lambda=-2 \ln L(\underline{s} ; y)+2 \ln L(\underline{m} ; \underline{y})
$$

will asymptotically behave like a $x^{2}$ distribution as the number of degrees of freedom goes to infinity. We can replace the unknown $m$ by $y$, in which case for Gaussian errors the second term cancels out, so that the estimator becomes the familiar $\chi^{2}$.

Data which are obtained by counting, however, follow a Poisson distribution:

$$
L_{P}(\underline{s} ; y)=\Pi_{i}: \operatorname{xp}\left(-s_{i}\right) \frac{\left(s_{i}\right)^{y_{i}}}{s_{i} !}
$$


as long as the only error in $y_{i}$ is a result of counting statistics. In this case, there are two approximations using a $\chi^{2}$ statistic:

$$
\begin{aligned}
& \text { Neyman's } \chi^{2}: \chi_{\mathrm{N}}^{2}=\sum_{i}\left(y_{i}-s_{i}\right)^{2} / y_{i} \text {, and } \\
& \text { Pearson's } \chi^{2}: \chi_{P}^{2}=\sum_{i}\left(y_{i}-s_{i}\right)^{2} / s_{i} .
\end{aligned}
$$

These statistics both reflect the fact that a Poisson distribution asymptotically converges to a Gaussian of width $y_{i}{ }^{1 / 2}$ (or $s_{i}{ }^{1 / 2}$ ) for $y_{i}, s_{i}>1$. These approximations have limitations, especially when the number of counts observed is small. Also, they do not preserve the area $A=\sum_{i} y_{i}$ in the fitted function: for $\chi_{\mathbb{N}}^{2}, A>\sum_{i} s_{i}$, and for $\chi_{P}{ }^{2}$, $A<\sum_{i} s_{i}$. This problem is severe in a case such as the fitting of a neutrino mass, which depends to a large degree on the area of the curve near the endpoint (where the counting rate, coincidentally, is very smal1). Therefore, an estimation parameter which is based on the Poisson distribution is preferable. Performing the same procedure on the Poisson likelihood function as on the Gaussian gives a Poisson " $x^{2}$ :

$$
\Xi^{2}-x_{\lambda}^{2}=2 \sum_{i} s_{i}-y_{i}-y_{i} \ln \left(s_{i} / y_{i}\right) \text {. }
$$

The function estimated using this parameter will preserve the total number of counts [Bak83, Hyn83] and therefore be much more suited to fitting the neutrino mass. $\Xi^{2}$ is in many ways similar to $x^{2}$. It has an expectation value at the minimum of 1 per degree of freedom, and the errors in the parameters can be estimated exactly as they would be with $x^{2}$. Therefore, since, for $y_{i}, s_{i} \rightarrow \infty, L_{P}\left(s_{i} ; y_{i}\right) \rightarrow L_{G}\left(s_{i} ; y_{i}\right)$, then:

$$
2\left[s_{i}-y_{i}-y_{i} \ln \left(s_{i} / y_{i}\right)\right] \approx\left(y_{i}-s_{i}\right)^{2} / y_{i} \text {, for } y_{i}, s_{i}>1 \text {. }
$$


In the current experiment, however, the error on data point $y_{i}$ is not determined solely by counting statistics, but has an additional component from the error in the beta monitor normalization. The error in the normalization factor is Gaussian and independent of the error in the number of counts observed in the detector, so the likelihood function is given by:

$$
L\left(s_{i}, y_{i}\right)=\int_{-\infty}^{\infty} d x \frac{1}{(2 \pi)^{1 / 2} N_{i} !} \exp \left(\frac{-\left(x_{i}-x\right)^{2}}{\sigma_{i}{ }^{2}}\right) x^{N_{i}} \exp (-x),
$$

Where $x_{i} A_{i}=1 / A_{i}$ is the reciprocal of the normalization, $\sigma_{i}$ is the error in $x_{i}$, and $N_{i}$ is the number of counts observed, $y_{i} x_{i}$. The expression is not amenable to use as a likelihood function because of constraints on computing resources. The properties of $x^{2}$ and $\Xi^{2}$ can be used, however, to produce an estimator which will work for the data. If the errors were completely Gaussian (a condition that is true for $y_{i}>$ 1), $\chi^{2}$ could be used, determined by:

$$
\left[\left(y_{i}-s_{i}\right) / \sigma_{i}\right]^{2}=\left[\left(y_{i}-s_{i}\right)^{2} / y_{i}\right] \times\left[y_{i} / \sigma_{i}^{2}\right]
$$

If the $x_{i}$ are about 1 where the number of counts is small (at the endpoint), and if the error in $x_{i} \leqslant 1$, then the $\Xi^{2}$ parameter can be modified to include the effects of the additional error by multiplying it by the same factor:

$$
\text { Modified } \Xi^{2} \approx 2 \cdot \sum_{i}\left[s_{i}-y_{i}-y_{i} \ln \left(s_{i} / y_{i}\right)\right] \times\left[y_{i} / \sigma_{i}^{2}\right]
$$


This approximation will work as long as $x_{i} \approx 1$ and $y_{i} / \sigma_{i}^{2} \approx 1$ when $y_{i}$ is small (less than about 25). A comparison of fits using $\chi^{2}$, modified $\Xi^{2}$, and a splice of $\chi^{2}$ and the unmodified $\Xi^{2}\left(\chi^{2}\right.$ was used for $\left.y_{i}>20\right)$ was performed using data generated by mockup, which was given statistics approximately equivalent to those of the actual data. The results are shown in Fig. 5.1 and Table 5-1. The modified $\Xi^{2}$ gives the best estimates, while $\chi^{2}$ consistently underfits the data and therefore overfits the neutrino mass. The splice method underestimates the mass since the method overestimates the area in the endpoint region compared to the rest of the spectrum. The modified $\Xi^{2}$ was also shown to produce very good confidence level estimates, based on a comparison of the predicted error in the neutrino mass to the observed standard deviation of the estimates. Hereafter, the modified $\Xi^{2}$ will be referred to as $\Xi^{2}$. The expectation value of $\Xi^{2}$ is 1 per degree of freedom. This characteristic gives a basis for a goodness of fit criterion, which is obtained exactly as for a $x^{2} \mathrm{fit}$. The probability of rejection of the fit can be determined from tables of the $\chi^{2}$ distribution. In general, however, the fit is adequate as long as $\Xi^{2}$ does not differ too much from 1 per degree of freedom. The contribution of each parameter $\xi_{j}$ to the fit can be determined from the amount that it changes the overall $\Xi^{2}$. If the change in $\Xi^{2}$ is "small", that is, the addition of a new parameter does not reduce $\Xi^{2}$ by many more than 1 (the expected reduction from the decrease in degrees of freedom), then the parameter is not needed for the fit. of course, some parameters, such as the neutrino mass, endpoint, background, and amplitude, must be present in any model of the spectrum, and should be fit if their values are not known. If the value 


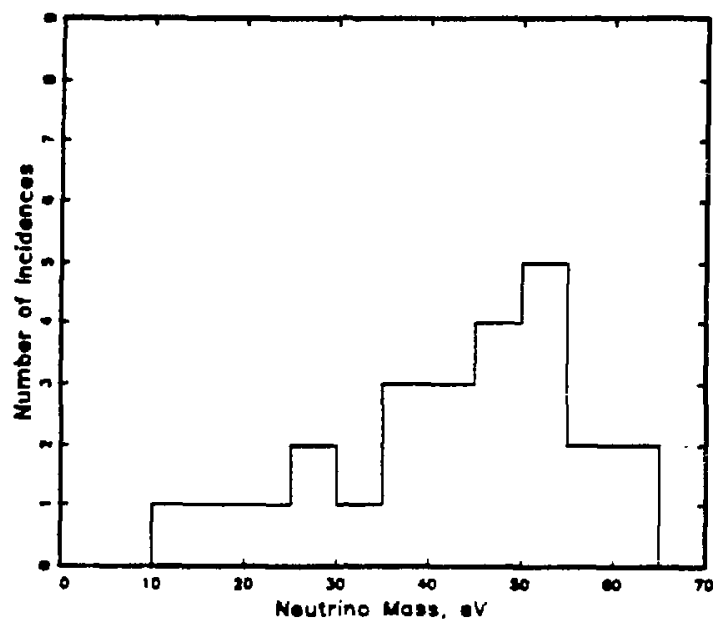

(a)

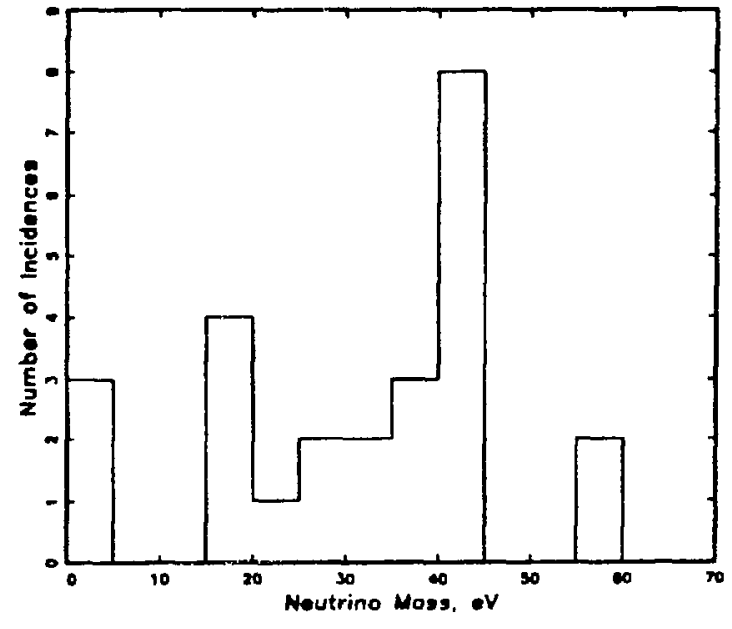

(b)

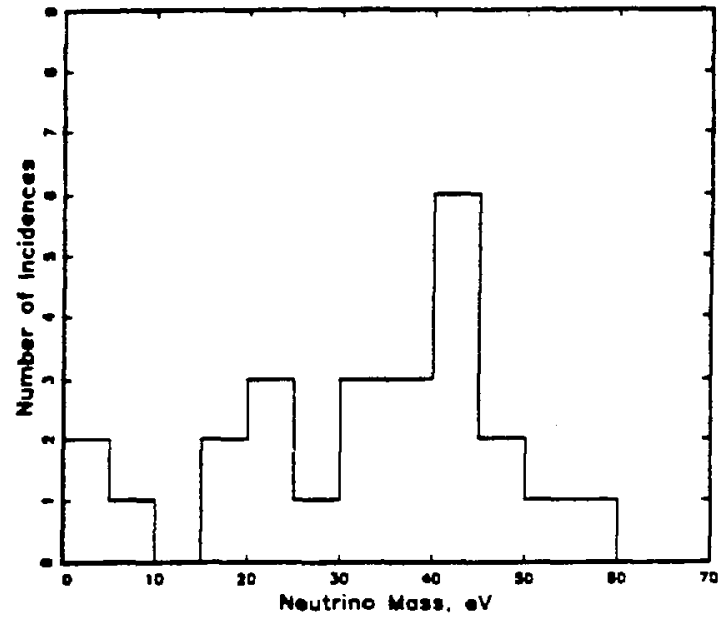

(c)

Fig. 5.1. Distributions of fitted neutrino masses for data created by Mockup. The expected neutrino mass is $35 \mathrm{eV}$. a) $\chi^{2}$ estimator. b) "Splice" estimator with crossover from Poisson to Gaussian statistics at 20 events. c) Maximum likelihood estimator $\Xi^{2}$. 
Table 5-1:

Tests of Various Estimators with Random Data

\begin{tabular}{|c|c|c|c|c|c|c|c|}
\hline \multirow[t]{2}{*}{ Data } & \multirow[t]{2}{*}{ Set } & \multicolumn{2}{|c|}{ Neyman's $x^{2}$} & \multicolumn{2}{|c|}{ Splice $(20)$} & \multicolumn{2}{|c|}{$\Xi^{2}$} \\
\hline & & Mass & Endpoint & Mass & Endpoint & Mass & Endpoint \\
\hline True & Value & 35.0 & 18580.0 & 35.0 & 18580.0 & 35.0 & 18580.0 \\
\hline 1 & & 61.7 & 18582.7 & 56.7 & 18581.7 & 54.4 & 18582.4 \\
\hline 2 & & 37.6 & 580.6 & 30.8 & 579.8 & 31.8 & 581.8 \\
\hline 3 & & 34.5 & 577.5 & 0.0 & 575.2 & 0.0 & 576.1 \\
\hline 4 & & 39.4 & 582.2 & 29.8 & 581.3 & 31.5 & 582.1 \\
\hline 5 & & 27.5 & 579.5 & 19.9 & 578.9 & 24.0 & 580.0 \\
\hline 6 & & 57.7 & 585.3 & 44.4 & 582.7 & 46.0 & 584.4 \\
\hline 7 & & 52.2 & 580.0 & 43.7 & 578.3 & 43.5 & 579.4 \\
\hline 8 & & 57.9 & 582.9 & 42.7 & 580.1 & 44.1 & 580.9 \\
\hline 9 & & 60.4 & 581.5 & 57.5 & 580.9 & 57.3 & 581.7 \\
\hline 10 & & 37.0 & 579.0 & 17.3 & 577.2 & 19.7 & 578.7 \\
\hline 11 & & 13.3 & 572.3 & 0.0 & 572.5 & 3.6 & 573.0 \\
\hline 12 & & 41.4 & 579.4 & 21.2 & 577.1 & 21.6 & 577.6 \\
\hline 13 & & 52.9 & 579.9 & 36.1 & 577.1 & 36.8 & 577.8 \\
\hline 14 & & 45.1 & 577.7 & 27.8 & 575.7 & 29.9 & 576.9 \\
\hline 15 & & 27.4 & 580.4 & 16.0 & 579.4 & 21.6 & 580.7 \\
\hline 16 & & 45.1 & 583.1 & 41.6 & 582.5 & 45.0 & 584.4 \\
\hline 17 & & 43.1 & 584.1 & 36.2 & 583.2 & 38.3 & 584.3 \\
\hline 18 & & 45.0 & 583.7 & 40.7 & 583.0 & 42.8 & 584.1 \\
\hline 19 & & 15.8 & 574.2 & 3.9 & 573.9 & 7.5 & 574.5 \\
\hline 20 & & 23.0 & 577.5 & 15.8 & 577.2 & 18.9 & 578.6 \\
\hline 21 & & 51.4 & 582.4 & 37.3 & 580.0 & 36.5 & 580.7 \\
\hline 22 & & 52.9 & 583.6 & 32.7 & 580.2 & 31.8 & 581.2 \\
\hline 23 & & 52.3 & 584.3 & 42.6 & 582.9 & 42.7 & 583.7 \\
\hline 24 & & 48.0 & 583.9 & 43.2 & 583.2 & 42.8 & 583.7 \\
\hline 25 & & 43.1 & 579.1 & 41.2 & 578.9 & 43.0 & 580.0 \\
\hline Mean & & 42.6 & 580.7 & 31.2 & 579.3 & 32.6 & 580.3 \\
\hline Std. & Dev. & 13.3 & 3.2 & 15.9 & 3.0 & 14.9 & 3.1 \\
\hline Predi & $\ddot{c}$ red $S$ & tandarc & Deviation & $\operatorname{rom} \Xi^{2}$ & & 14.5 & 3.2 \\
\hline
\end{tabular}


of any of these can be determined a priori to better precision than would be possible from the experimental data, then the parameter can be fixed and the effect of its covariance with other parameters in the fit can be eliminated.

The fitting programs output a set of residuals, which can be examined for systematic variations with energy, in order to detect the presence of inadequacies in the fitting model or systematic errors. A Kurie plot of the data and best fit is also output.

\section{Error Analysts}

To determine the error on a parameter $\xi_{j}$, it is stepped through a series of fixed values while all other parameters are fit. The parameter $\xi_{j}$ is varied in each direction until $\Xi^{2}$ increases by 1 , and these limits now represent the $1 \sigma$ limits on that value. These limits include the uncertainty from covariance with other parameters in $\xi$ which also have uncertainties. The $2 \sigma$ limits are where $\Xi^{2}$ increases by 4 , etc. This procedure works on any likelihood function obtained from the likelihood ratio theorem [Ead71].

These uncertainties can be estimated another way if the $\Xi^{2}$ hypersurface is nearly parabolic at the minimum, as is the case when a parameter varies linearly with the statistics. The $\Xi^{2}$ parabola can be expanded to first order, and an approximation to the curvature matrix $\underline{\alpha}$ obtained:

$$
\alpha_{j k} \approx 1 / 2\left(\partial^{2} \bar{\Xi}^{2} / \partial \xi_{j} \partial \xi_{k}\right)
$$


Then the covariance matrix (also known as the error matrix) is given by:

$$
\epsilon=\underline{x}^{-1}
$$

Using this matrix, the error in parameter $\xi_{j}$ is given by:

$$
\sigma^{2} j \mathrm{j}=\sigma^{2}\left(\xi_{j}\right)=\epsilon_{j j}
$$

In the case of the parameters being fitted in this case, these approximations are not very good. For example, $\mathrm{m}_{\nu}$ is expected to vary as the number of "missing" counts cubed, so a linear approximation is inadequate. The error matrix does have one use, however: it can generate an approximation to the correlation matrix $\mathrm{I}$ :

$$
r_{j k}=\sigma_{j k}^{2} / \sigma_{j} \sigma_{k} \approx \epsilon_{j k} /\left(\epsilon_{j j} \epsilon_{k k}\right)^{1 / 2} .
$$

Even though the correlation matrix is determined from an inadequate approximation for the errors, it nevertheless can be used to get some idea of the correlations between the elements of $\xi$.

\section{Systematic Errors}

Those parameters which are not varied in the fits may also have errors associated with them, either statistical or systematic. These errors must be determined independently of the fit. At issue is the effect of these errors on the neutrino mass. This effect can be determined simply, using the same fitting programs used to obtain the mass. A new value for the parameter or parameters of interest is used to generate the theoretical spectra, and a best fit to the fitted parameters is obtained. The difference between the neutrino mass in the 
new fit and that in the old is the systematic change which results from the new value for the parameter(s) of interest. This method can be used to determine the systematic error in the mass from many different sources, including:

Resolution Function

Energy Loss in the Source

Acceleration Gap Effects

Final State Effects

Placing all of the non-fitted parameters at extreme values gives an extreme upper limit to the effect of these parameters on the neutrino mass. Because of the nonlinearity of the effect of the neutrino mass on the beta spectrum, the effects of the systematic errors will vary with the neutrino mass, so they cannot simply be added in quadrature. As was shown in Chapt. 2, the neutrino mass squared is approximately linear in the statistics, so a good approximation of the effect of the systematic errors is to add the changes in $m_{\nu}{ }^{2}$ in quadrature as long as the errors are independent.

A search for the presence of systematic errors which are not immediately apparent from an inspection of the residuals plot can be performed by fitting different sections of the data to ensure that the parameters do not systematically vary with energy. This is most practically accomplished by holding the efficiency coefficients constant while reducing the energy range of the fit. The efficiency coefficients are held constant so that the error in them, which gets very large when small energy ranges are fit, does not conceal a small systematic variation in another parameter. This method is still sensitive to 
systematic errors in the efficiency coefficients, even though they are not fit, since the systematic error will appear in another parameter which is covariant with them, most likely the endpoint.

\section{Determination of the Resolution Function}

The spectrometer resolution function is determined by fitting the observed ${ }^{83} \mathrm{~m}_{\mathrm{Kr}}$ 1ineshape. The line is fit to a skewed Gaussian, given by :

$$
P(x)=A \exp \left(\frac{-(x-\mu)^{2}}{2(\sigma+\lambda(x-\mu))^{2}}\right),
$$

where $\mathrm{A}$ is the amplitude, $\mu$ is the "mean", $\sigma$ is the "width", and $\lambda$ is the skewness. For large values of the skewness $\lambda$, the mean and width of the spectrum can be very different from those which would be expected in a normal Gaussian, namely $\mu$ and $\sigma$. This is not the case for the data presented here, however. The function is convoluted with the predicted shakeoff spectrum for the $\mathrm{Kr}$ line and with the window width of the detector, and then fitted to the observed $\mathrm{Kr}$ lineshape, using a nonlinear least squares algorithm [IMS03]. The error in the width of the peak and in the position of the peak can be determined in a manner identical to the determination of errors, in the tritium spectrum. There is a component of scattering in some of the $\mathrm{Kr}$ spectra which results from other gases in the source which build up as the $\mathrm{Kr}$ is recirculated. No attempt was made to calculate this effect, but rather, the peak is fit above a pedestal of background which is not considered part of the resolution function. This procedure tends to underestimate the peak 
width, but, since the ratio of peak height to the pedestal is typically greater than 20 to 1 , and since the high-energy side of the peak is observed to fall off as a Gaussian, the effect on the width is small. The actual width of the peak was taken to be the average of the fitted width with the pedestal and the width the peak would have with no pedestal, obtained by assuming the same peak shape but no background. The difference in widths determined by these two methods was always less than $.5 \mathrm{eV}$. No correction was applied if the krypton was not recirculated.

The shakeup and shakeoff spectrum is determined as follows. The electronic configuration of $\mathrm{Kr}$ is $1 \mathrm{~s}^{2} 2 \mathrm{~s}^{2} 2 \mathrm{p}^{6} 3 \mathrm{~s}^{2} 3 \mathrm{p}^{6} 3 \mathrm{~d}^{10} 4 \mathrm{~s}^{2} 4 \mathrm{p}^{6}$. The electron line observed is the $K$ internal conversion line of the $32.14 \mathrm{keV}$ gamma from $83 \mathrm{~m}_{\mathrm{Kr}}$. The total shakeoff (and shakeup) probability has been calculated by Carlson and Nestor [Car73]. The shakeup occurs mostly from the $4 \mathrm{p}$ subshell, since electrons from shells further in have a much higher probability of shakeoff than shakeup [see Car73]. The relative intensity of the $4 \mathrm{p}$ shakeup line to the primary has been measured by Spears et al. for a vacancy in the $3 p$ or $3 \mathrm{~d}$ subshells [Spe74], and the shapes of the shakeoff spectra from inner shells have been calculated by Levinger [Lev53]. These shapes, shown in Fig. 5.2, get narrower as the principal quantum number of the shaken-off electron increases. For this approximation, the $2 \mathrm{~s}$ and $2 \mathrm{p}$ shapes were used, although the electrons of interest come mostly from the $4 \mathrm{~s}$ and $4 \mathrm{p}$ subshells. The widths of the Levinger spectra are, in any case, negligible compared to the width of the spectrometer resolution. The 
width of the primary line is just the width of the $K$ vacancy, which is given by:

$$
W \approx 1.73 \times 10^{-6} \mathrm{z}^{3.93} \mathrm{eV} \quad[\operatorname{Bam} 72] .
$$

For $\mathrm{z}-36$ this yields a width of $2.26 \mathrm{eV}$. A Lorentzian of this width was used for both the primary peak and the $4 \mathrm{p}$ shakeup peak. With a vacancy in the $K$ shell, the binding energies of the $\mathrm{Kr}^{+}$electrons are very similar to those of the $\mathrm{Rb}$ atom, with an added potential because the atom is an ion. This added potential was calculated by Carlson and Nestor [Car73]. Assuming that the relative intensity of the $4 \mathrm{p}$ shakeup peak to the total shakeoff and shakeup probability would remain unchanged for a is vacancy compared to $a 3 p$ or $3 d$ vacancy, the spectrum was calculated, with the result shown in Fig. 5.3.

\section{Acceleration Gap Correction}

Tritium that decays in the acceleration gap decays at a potential different from that determined for the rest of the tube. This effect can distort the spectrum if the amount of tritium decaying in this region is large. The potential in the acceleration gap was calculated using the CHARGe program written by K. Crandall [Cra84,Cra66]. This program numerically calculates the potential in space for an input geometry of conducting surfaces at various potentials. The potential thus determined at a point $z$ (in $\mathrm{cm}$ ) along the axis of the rings is fit wel1 by:

$$
\left(v_{0}-V\right) \approx 0.20 v_{0} \exp \left[1.206\left(z-z_{0}\right)\right] \quad \text {, for } v \geq v_{0} / 2
$$




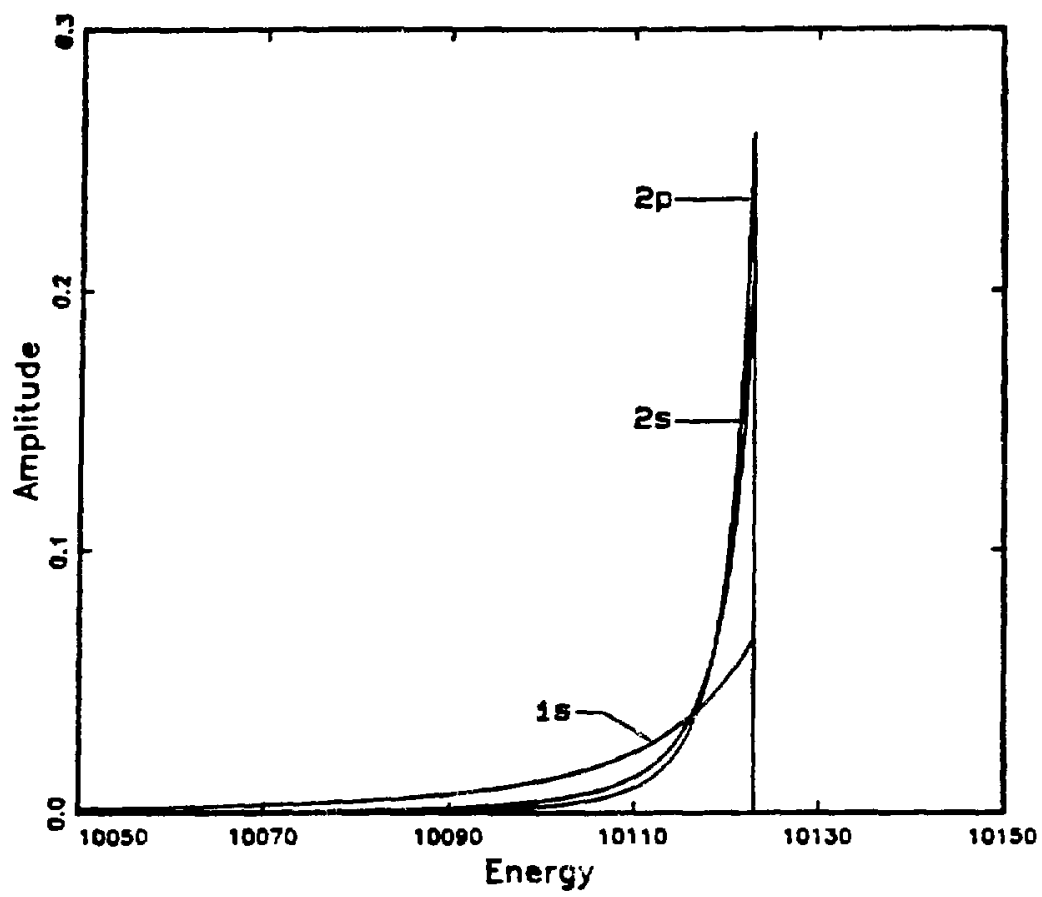

Fig. 5.2. Shakeoff energy spectra for krypton, calculated by Levinger [Lev53], normalized to unit area. Assumed central energy of $10150 \mathrm{eV}$ with transition energy to continuum of $26 \mathrm{eV}$.

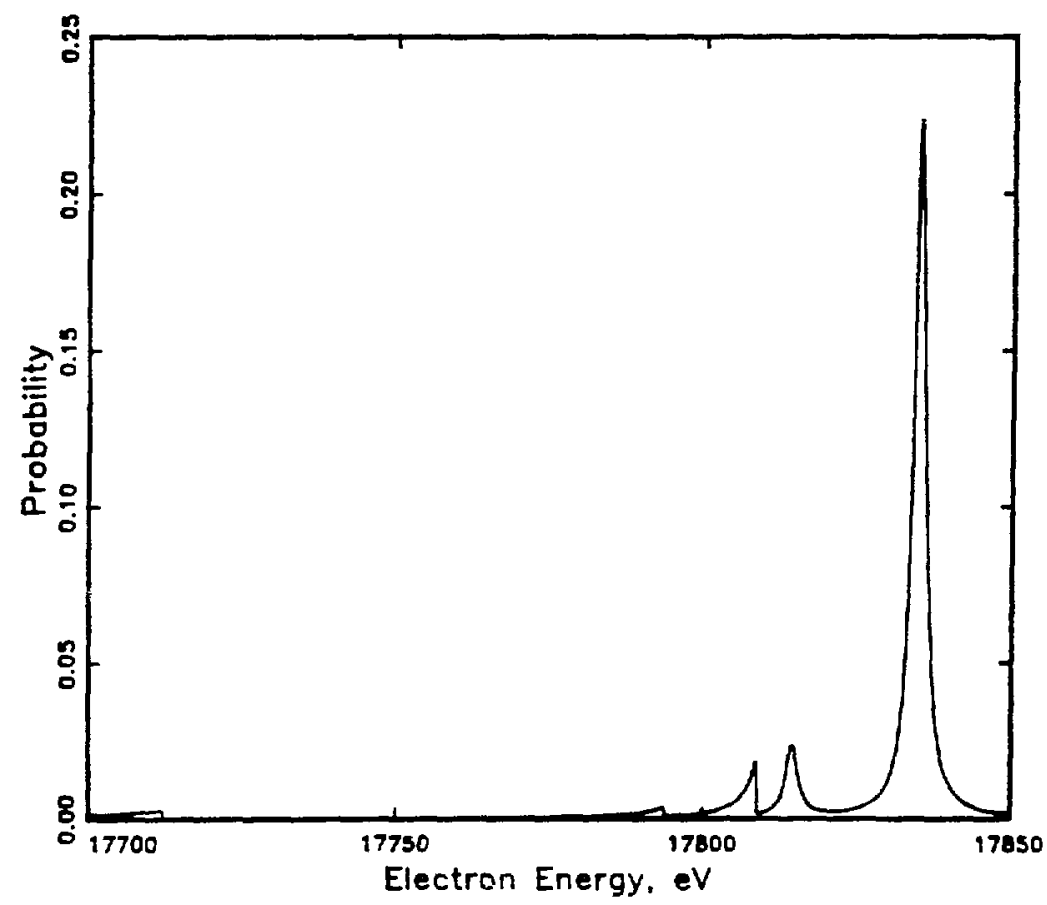

Fig. 5.3. The energy spectrum of shakeoff from krypton, normalized to unit area. 
where $z_{0}$ is the position of the end of the rings, and $v_{0}$ is the potential of the source tube. Assuming that the pressure is constant as a function of $z$ (which should be true in the rings), then the amount of gas which can decay between potentials $v_{0}-V$ and $v_{0}-(V+\Delta V)$ is proportional to the length $\Delta z$ for which that potential exists. Differentiating the expression above gives:

$$
\mathrm{dV} / \mathrm{dz}=-1.206\left(\mathrm{~V}_{0}-\mathrm{V}\right) \text {, so that } \Delta \mathrm{z} \approx \Delta \mathrm{V} / 1.206\left(\mathrm{~V}_{0}-\mathrm{V}\right)
$$

Since the effect of the acceleration gap only defends on $v_{0}-v$, it does not depend on the voltage applied as long as the minimum voltage applied during the measurement of a spectrum is more than half the maximum. The ratio of tritium per centimeter in the gap to the total source strength is known from the pressure in the pump tank and the temperature of the source tube. For each value of $V_{0}-v$, a spectrum shifted by $-V$ whose amplitude is the relative probability of decaying between $V_{0}-V$ and $V_{0}-(V+\Delta V)$ is added to the theoretical spectrum. Usually the tritium pressure in the pump tank is proportional to the density of tritium in the source tube, so the effect on the neutrino mass is constant. For a pressure of $1 \times 10^{-5}$ torr in the grids and a total integrated density of $10^{16}$ atoms $/ \mathrm{cm}^{2}$ in the source the effect on a neutrino mass of $35 \mathrm{eV}$ is $.1 \mathrm{eV}$. In actual runs, the ratio of pressure in the tank to source density is lower, so this is an upper limit. Therefore, this effect is neglected. 


\section{Determination of Final States}

The final states for molecular tritium were calculated by Martin and Cohen [Mar85] at Los Alamos. Using an expansion for the electronic wavefunctions composed of Cartesian Gaussians, they calculated the first few bound states, and a series of eigenvectors whose energies lie in the continuum. The latter eigenvectors do not represent the actual shape of the continuum, but they do approximate the spectral moments $\mathrm{S}(-\mathrm{k})$ :

$$
S(-k)=\int d E E^{-k} \frac{d P}{d E} \approx \sum_{i=1}^{N} P_{i} \Delta E_{i}^{-k}
$$

where $P_{i}$ is the probability of state $i, \Delta E_{i}$ is the energy loss in the transition to state $i, N$ is the number of states in the continuum, and $\mathrm{dP} / \mathrm{dE}$ is the actual distribution. The approximation is good for $2 \mathrm{M}$ moments, where $M<N$. The moments can then be inverted to give an approximation to the true distribution. They can also be inverted to give $M$ states which exactly reproduce the first $2 M$ moments of the approximation to the true distribution. Since in the Los Alamos experiment the resolution is wide enough to smear out any fine structure in the continuum, such an approximation was used. Six states in the continuum were generated by inversion of the moments. These states exactly reproduce the first 12 spectral moments of the actual distribution. They also approximate the first few positive moments well. The neutrino mass is most sensitive to the first few moments of the final state spectrum, so this approximation is certainly adequate. The approximation was tested against a more elaborate continuum 
distribution, consisting of the continuous spectrum binned into $3 \mathrm{eV}$ bins, by fitting the neutrino mass of a mockup spectrum using each set of final states. No change in the fitted neutrino mass was observed.

The calculations of Kolos et al. [Ko185] are in good agreement with these calculations, even though a different method is used which is more sensitive to the initial wavefunction approximations. The Kolos calculations are much more focused on trying to understand the fine details of the spectrum, including rotational and vibrational effects, which were neglected in the Los Alamos analysis.

The bound atomic final states, including electron exchange effects, have been calculated by Williams and Koonin [Wi183]. They found that the states differed very little from those obtained using the "sudden" approximation. The continuum, in the sudden approximation case, will just vary as $\mathrm{dP} / \mathrm{dE} \propto \mathrm{E}^{-7 / 2}$. The final states for atomic and molecular tritium are shown in Fig. 5.4. The actual final states used in the calculations are given in Table 5-2.

\section{Determination of Scattering in the Source}

The effect of energy loss from scattering in the source was also calculated. The electrons in the source fall into two categories: either they escape the source with a small probability of scattering or they are trapped in the source and can only be detected when they scatter into the solid angle which escapes.

The energy spectrum of the singly scattered electrons was estimated using data obtained by Ulsh et al. [U1s74] and Geiger [Gei64], both of whom measured the differential inelastic cross-sections for 

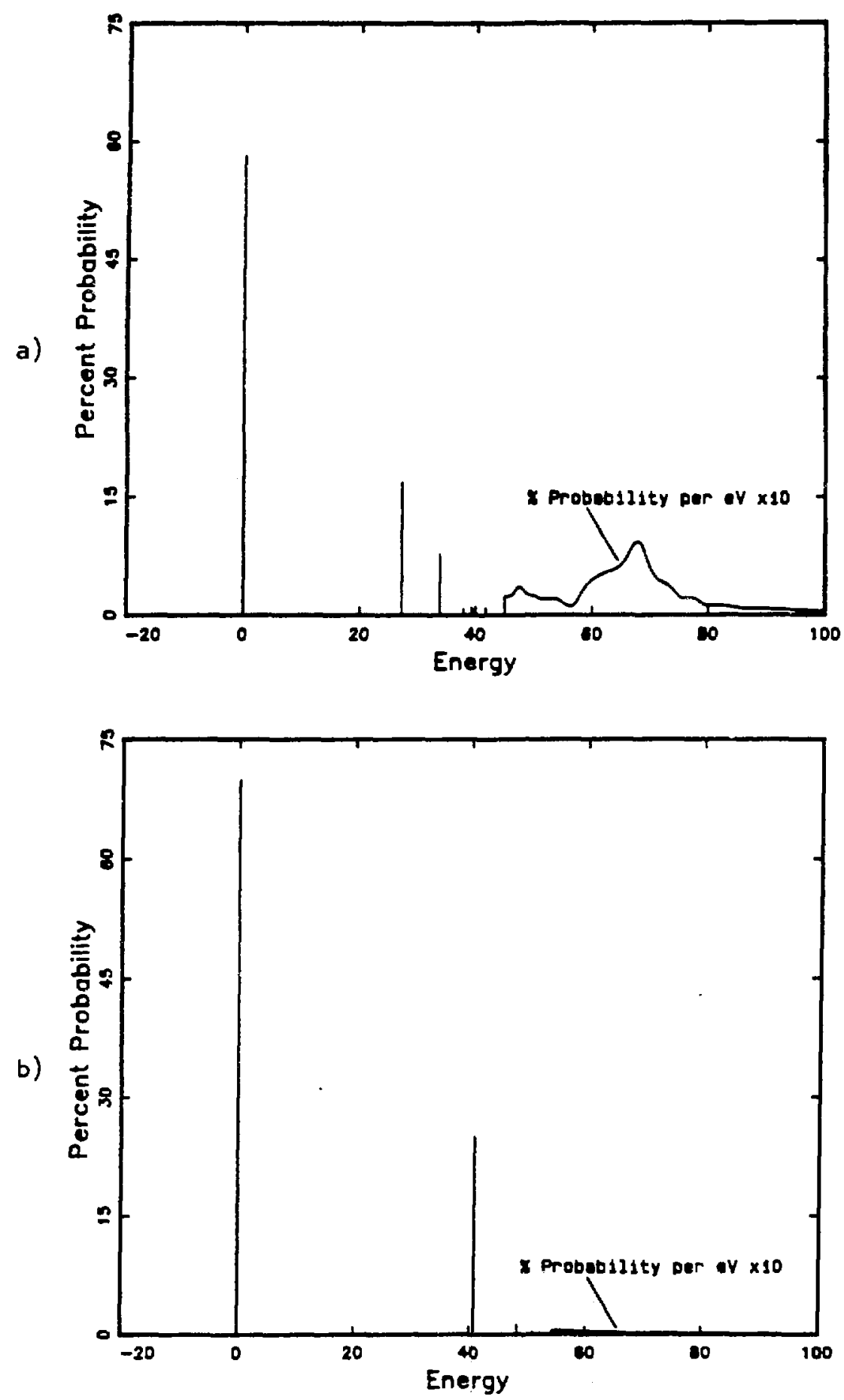

F18. 5.4. Final state spectra for gaseous tritium sources. a) Molecular tritium or tritium hydride. b) Atomic tritium. 
Tabie 5-2:

Final States Used in the Analysis

$\begin{array}{cc}\text { Energy Loss } & \text { Amplitude } \\ 0.0 & .5822 \\ 27.29 & .1675 \\ 33.89 & .0787 \\ 37.96 & .0081 \\ 38.82 & .0001 \\ 39.38 & .0092 \\ 41.75 & .0089 \\ 46.03 & .0143 \\ 51.71 & .0160 \\ 65.28 & .0789 \\ 74.59 & .0297 \\ 88.07 & .0061\end{array}$


$25 \mathrm{keV}$ electrons on $\mathrm{H}_{2}$. The total cross-sections for inelastic scattering, ionization, and discrete excitation as a function of incident electron energy were calculated by Liu [Liu73] from measurements made by a number of investigators. The total cross-section for inelastic scattering is given by:

$$
\sigma_{\text {tot }}=\left(4 \pi a_{0}{ }^{2} z^{2} \mathrm{R} / \mathrm{T}\right)[1.5487 \ln (\mathrm{T} / \mathrm{R})+2.2212],
$$

where $a_{0}$ is the Bohr radius, $z$ is the charge of the incident particle, $R$ is the Rydberg energy $(13.6 \mathrm{eV})$, and $T$ is the kinetic energy of the incident electron. The total cross section is about $3.45 \times 10^{-18} \mathrm{~cm}^{2}$ for $18.5 \mathrm{keV}$ electrons. Making the approximation that this is all from excitations to either discrete states or to the continuum (and not to quasi-bound dissociative states), then about 408 of the strength goes to discrete states and 608 to the continuum. The discrete states were approximated as a Lorentzian with width $1.7 \mathrm{eV}$, based on the shapes observed by Geiger [Gei64]. An empirical fit to the observed shapes of the continuum at different angles [U1s74] gives the following energy spectrum in the continuum:

$$
\frac{\mathrm{dP}(E)}{\mathrm{dE}} \propto \int_{\theta_{\min }}^{\pi / 4} \mathrm{~d} \theta \frac{1}{\sin ^{4} \theta} \exp \left(\frac{-\left(E-E_{0} \sin ^{2} \theta\right)^{2}}{2\left(.02435 \mathrm{E}_{0} \sin \theta\right)^{2}}\right), E>15.45 \mathrm{eV}
$$

where $E_{0}$ is the electron energy $(18.6 \mathrm{keV}$ in this case). The resulting spectrum normalized to unit area is shown in Fig. 5.5. The spectrum was observed to be insensitive to the minimum angle $\theta_{\min }$, since most inelastic scatters in the forward direction go to discrete states. In 


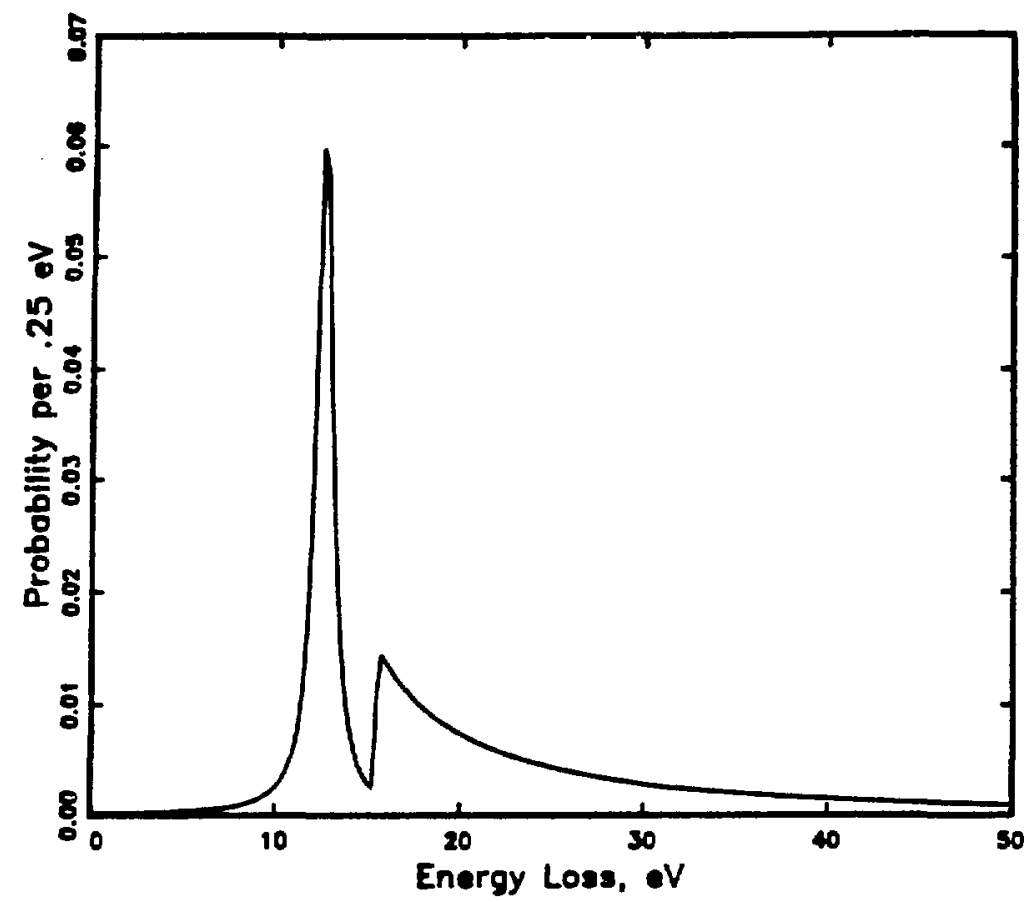

Fig. 5.5. Energy loss spectrum of inelastically scattered electrons in tritium gas, normalized to unit area.

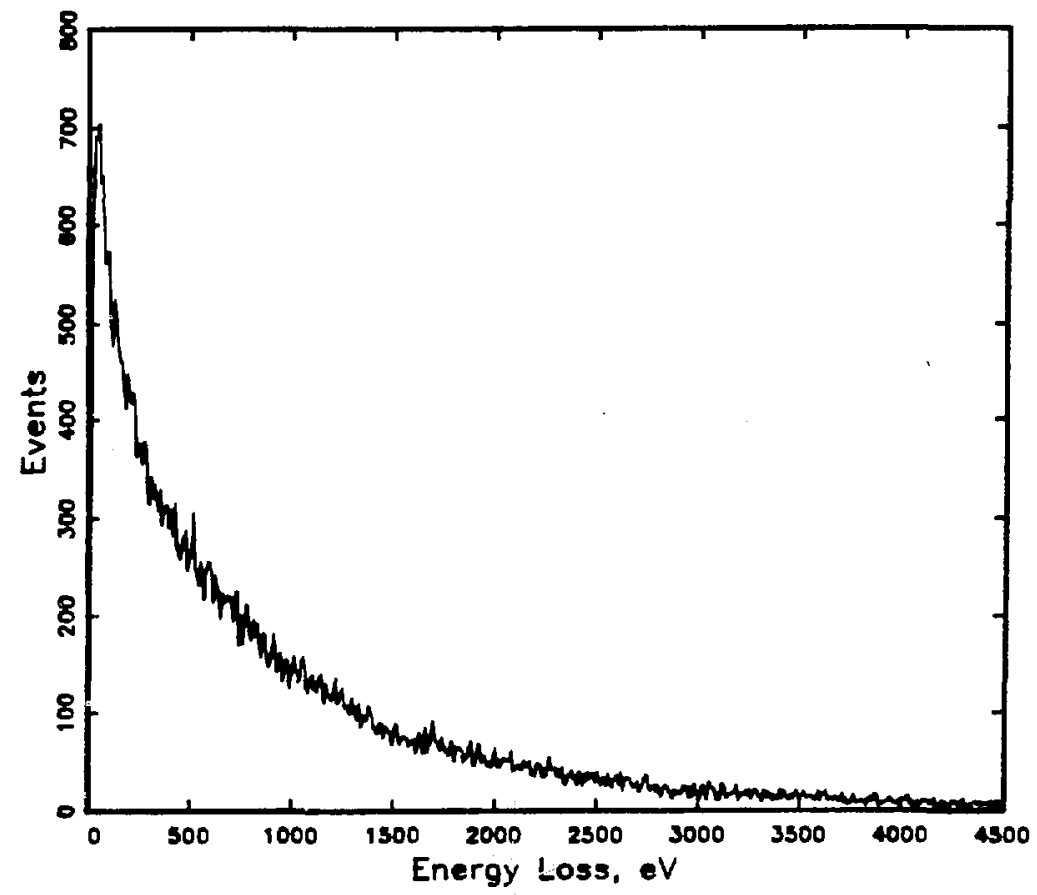

FIg. 5.6. Energy loss spectrum of trapped particles, calculated by Monte Carlo sinulation of 50,000 particles. The energies have been binned into $9 \mathrm{eV}$ wide bins. The spectrum continues down to much higher energy losses, which are not shown. The average energy loss in this simulation was $1009 \mathrm{eV}$. 
this case $\theta_{\min }-0.1^{\circ}$ was used. The probability of scattering $n$ times, assuming that the scatter does not change the particle's trajectory significantly, is given by a Poisson probability distribution:

$$
P(n)=\exp (-\mu) \frac{\mu^{n}}{n !},
$$

where $\mu$ is the average number of scattering events per particle. For a total scattering probability of 58, the probability of multiple scattering is .138. Therefore, for the particles which were not trapped, the scattering was assumed to be completely single scatters. In the approximation of single scattering, the only parameter of interest is the mean of the of the path length times density distribution, which, when multiplied by the cross-section for inelastic scattering, gives the total probability of scattering. Elastic scattering, which has a much smaller cross section than does inelastic scattering [see Uls74], and a maximum energy loss of only $3 \mathrm{eV}$, was ignored.

The average path length times density in molecules/ $\mathrm{cm}^{2}$ for those particles which were not trapped, which will be abbreviated $\langle p z\rangle$, was determined by approximating the magnetic field in the source as a uniform field with a perfectly elastic pinch at one end. This approximation is justified because the density of gas at the magnetic pinch is less than 18 the density in the center of the source tube, so that the contribution to $\langle\rho z\rangle$ of the turns near the pinch is negligible. For any particle emitted with opening angle $\theta$ (the angle between the 
magnetic axis and the direction of the particle), the path length is given by:

$$
\rho z(\theta, z)=\int \frac{\rho(z)}{\cos \theta} d z
$$

where $\rho(z)$ is the density in molecules $/ \mathrm{cm}^{3}$ at $z$, a quantity which does not depend on the radius $r$. This expression can be simplified by observing that the sum of the path lengths of a particle originating with angle $\theta$ and angle $2 \pi-\theta$ is only a function of $\theta$ :

$$
\rho z(\theta, z)+\rho z(2 \pi-\theta, z)=\frac{2 \rho z_{t o t}}{\cos \theta}
$$

where $\rho z_{\text {tot }}$ is the total integrated source density in molecules $/ \mathrm{cm}^{2}$. Integrating this over the solid angles gives:

$$
\langle\rho z\rangle=\int_{\theta=0}^{\theta} \rho z_{\text {tot }} \tan \theta \mathrm{d} \theta=-\rho z_{\text {tot }} \ln \left(\cos \theta_{\max }\right) .
$$

This expression diverges if integrated up to $\theta=\pi / 2$, but it only needs to be integrated up to so: maximum angle $\theta_{\max }$, which is defined as the angle above which all the particles are trapped: all the particles emitted with $\theta>\theta_{\max }$ are trapped in the source and must multiply scatter to escape. For this approximation, the average trapping angle was used as $\theta_{\max }$ (the derivation of the average trapping angle is given below). The total probability of single scattering is given by:

$$
P(\text { scattered })=\langle\rho z\rangle \sigma_{\text {tot }}[1-P(\text { trapped })]
$$

Because of the configuration of the field in the solenoid, a substantial fraction of the emitted beta particles were trapped in the 
source, and could only get out through multiple scattering. The "bump" in the field responsible for this trapping was located near the acceleration gap. The number of particles trapped was determined by integrating the solid angle in which particles are trapped at each point along the tube multiplied by the pressure at that point. The minimum angle trapped in a field $B_{z}$ with a pinch field of $B_{0}$ is given by:

$$
\tan \theta_{\min }=\left(B_{0} / B_{z}-1\right)^{-1 / 2} \text {, }
$$

and the relative solid angle of the trapped particles is given by $\cos \theta_{\min }$. So the number of particles trapped is given by:

$$
P(\text { trapped })=\frac{\int d z \rho(z) \cos \theta_{z}}{\int d z \rho(z)} \text {, }
$$

where $\theta_{z}$ is the minimum angle trapped by the field at $z$, and $\rho(z)$ is the pressure at $z$. The percentage of particles trapped is independent of the pressure in the tube, and only depends on the distribution of the pressure in the tube and the magnetic fields. The particles must scatter out to an angle less than $\theta_{z}$ to be detected in the spectrometer. The average trapping angle can also be determined, by:

$$
\left\langle\theta_{2}\right\rangle=\frac{\int \mathrm{d} \theta_{2} \mathrm{P}\left(\theta_{2}\right) \theta_{2}}{\int \mathrm{d} \theta_{2} \theta_{2}},
$$

where $P\left(\theta_{2}\right)$ is the probability of an electron decaying at a point in the source tube with trapping angle $\theta_{z}$. This average angle was used as the 
maximum angle for the singly scattered particles (see above). The calculations for the source revealed that almost all the particles decay with trapping angles between $81^{\circ}$ and $85^{\circ}$, with an average trapping angle of $83.2^{\circ}$, which was used to determine the single scattering probability. The total trapping probability, P(trapped), was determined to be 10.18 .

The energy loss spectrum of the trapped particles was calculated by performing a Monte Carlo simulation of the multiple scattering in the source. A particle was generated at a random angle inside a random trapping angle and scattered until it escaped. The energy loss spectrum for the scatters was as described above, with the differential crosssection for scatters to the continuum approximated by Moller scattering [Mol32]. Scatters to discrete states were simulated by a single angle, an approximation which is valid because these scatters take place only at small angles. The resulting energy loss spectrum for 50000 particles is shown in Fig. 5.6. This spectrum was insensitive to the angle used for discrete scatters. Adding the single-interaction energy loss spectrum to the multiple-interaction spectrum gives the total energy loss spectrum. 


\section{Chapter 6:}

\section{Results}

\section{Data Sets}

Two scans of the tritium spectrum were taken, labeled as data sets TBD3, and TBD4-1. The data were obtained in January and March 1986. Each set will be treated independently, since the resolution, background, and count rate were different. However, the results can be combined by adding the $\Xi^{2}$ for each as a function of neutrino mass, which gives an overall estimate of confidence intervals.

These data sets were not the only data taken. Earlier data had been obtained, but the resolution functions were not known and the beta monitor normalization was not fully implemented, so they were not used to determine the neutrino mass.

Data Set TBD3:

The first data set was taken between Jan. 14 and Jan. 18, 1986 . The data reduction had to be split into two parts because a new bcttle of tritium was inserted in the middle of the run. The first part, which contained about 258 of the points, had a count rate which dipped by the end to half the average rate in the second part, so that the points from the first portion contributed relatively little to the statistics. The beta monitor ratio $\mathrm{R}_{\beta}$ (see Chapt. 5) varied somewhat during the run, probably the result of an inadequate linear gate stretcher module, which was replaced after the run. These variations had a long time constant, 


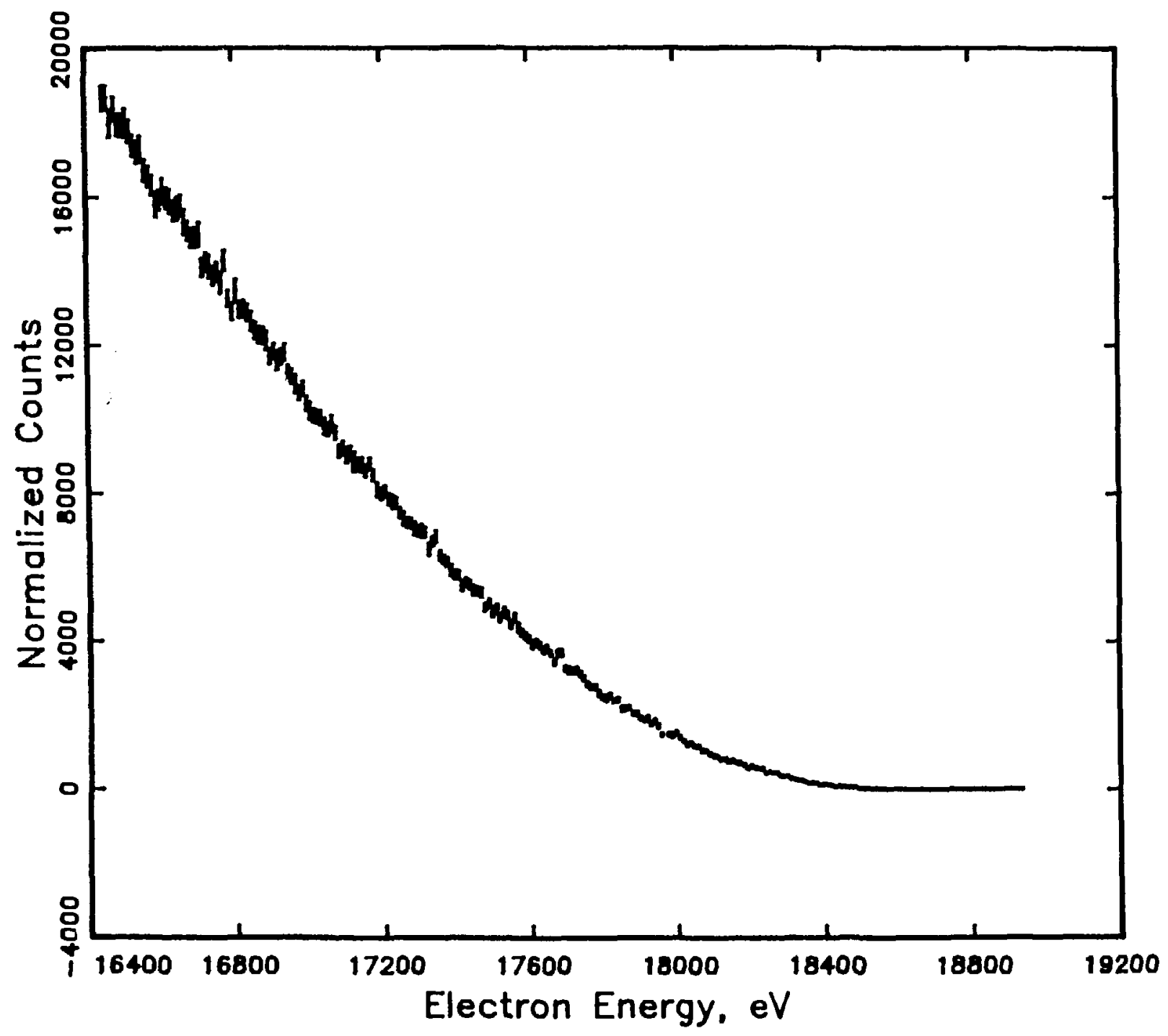

FIg. 6.1. Normalized data from run TBD3.

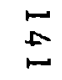


on the order of hours, so the calibration points taken before and after each two data points were used to determine the value of $R_{\beta}$ for the data points. A plot of the normalized data is shown in Fig. 6.1.

A series of krypton resolution scans were taken after the data set was completed. These scans were taken with the krypton in single-pass mode, which means that there was very little scattering of the conversion electrons from gas in the source. Therefore, the width of the resolution function obtained by fitting these spectra was not adjusted for the apparent background introduced by scattering. The scans showed that the long-term stability of the resolution function was quite good, and they were averaged to produce a single peak which could be fit with good statistics. The fit to the krypton data is shown in Fig. 6.2 and Table 6-1. The error in the width of the resolution function was determined by varying it while fitting all the other parameters, until the value of $x^{2}$ increased by 1 . This resulted in an error in the width of $.51 \mathrm{eV}$. The systematic error in the width was determined by varying the shakeoff amplitude by $10 \%$, a process which resulted in a $.3 \mathrm{eV}$ increase in the width, and by adding an error from possible long-term voltage variations in the source tube, estimated to have a FWHM of about $1 \mathrm{eV}$, or a width contribution of $.4 \mathrm{eV}$. Adding these terms in quadrature gives an overall error in the width of $.71 \mathrm{ev}$. The average integrated source strength for the second part of this run was determined to be $7.8 \times 10^{15}$ molecules $/ \mathrm{cm}^{2}$, which gives a single scattering probability of 5.158, given a trapping probability of 10.18 . This scattering probability was used for the fit to the whole scan. An estimate of the systematic error in this parameter was obtained by 

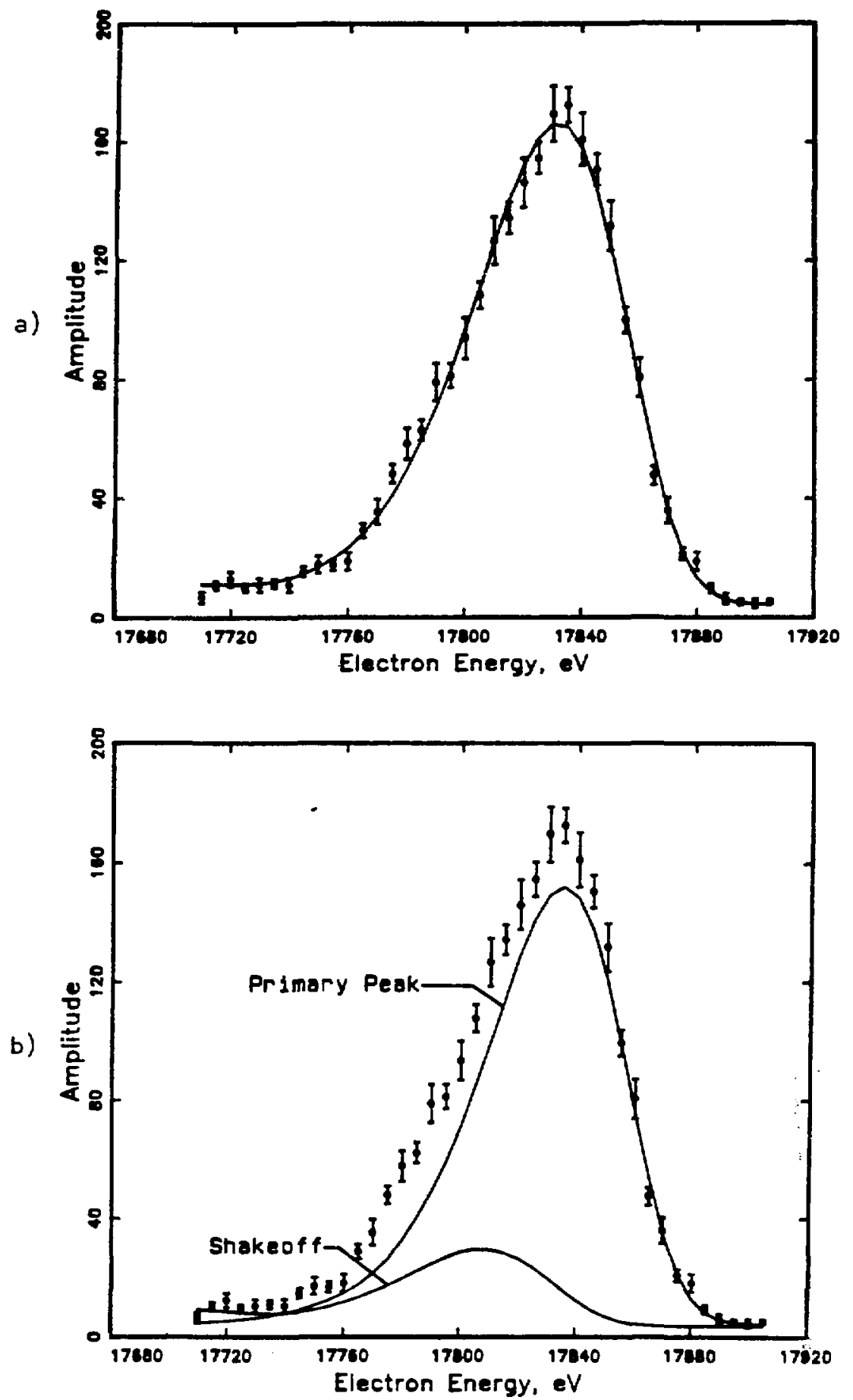

Fig. 6.2. Fit to the krypton peak for TBD3. a) overall fit. b) Components of the spectrum, including the main peak and the shakeoff spectrum. 
Table 6-1:

Krypton Fits

$$
\begin{aligned}
& \text { Data Set: } 3-1 \\
& \text { Centroid }=17835.8 \mathrm{eV} \\
& \text { Width }=22.241 \mathrm{eV} \\
& \text { Skewness }=-0.1334 \\
& \text { Amplitude }=9.6123 \mathrm{eV}^{-1} \\
& \text { Background }=0.159 \mathrm{eV}^{-1} \\
& x^{2} / \text { DoF }=40.5 / 35
\end{aligned}
$$

Data Set: 4-1a

Centroid $=17834.4 \mathrm{eV}$ Width $=15.473 \mathrm{eV}$

Skewness $=+0.0190$

Amplitude $=28.33 \mathrm{eV}^{-1}$

Background $=1.087 \mathrm{eV}^{-1}$

$x^{2} /$ DOF $=29.0 / 28$
Taken After TBD3; Average of 4 scans. for Spect. Energy $=25945 \mathrm{eV}$
Taken Before TBD4-1

for Spect. Energy $=25967 \mathrm{eV}$
Taken After TBD4-1

for Spect. Energy $=25967 \mathrm{eV}$

$$
\begin{aligned}
& \text { Centroid }=17835.9 \mathrm{eV} \\
& \text { Width }=16.188 \mathrm{eV} \\
& \text { Skewness }=-0.0137 \\
& \text { Amplitude }=28.79 \mathrm{eV}^{-1} \\
& \text { Background }=.995 \mathrm{eV}^{-1} \\
& x^{2} / \text { DOF }=38.5 / 28
\end{aligned}
$$


adding 18 to the scattered intensity as an estimate of possible errors in the approximation of path length times density and in the determination of source tube pressure. The error in the trapping probability was estimated to be .28 , added coherently with the error in the single scatters. The total resolution function, including the effects of window width and energy loss, is shown in Fig. 6.3. The full width at half maximum (FWHM) of this peak is about $54 \mathrm{eV}$.

The best-fit parameters for this data set are shown in Table 6-2. The best-fit real neutrino mass is zero. The residuals plot for zero neutrino mass is shown in Fig. 6.4, and a Kurie plot of the data and this fit is shown in Fig. 6.5. For this data set, and for the other two as well, it was found that either the linear or the quadratic efficiency term fit the data well, but that both were not needed. In this case, adding the linear term reduced the best-fit $\Xi^{2}$ by 0.5 , not even compensating for the decrease in degrees of freedom. Since the sign of the coefficients is negative, the linear term will tend to give a lower neutrino mass. The linear term alone also gave systematic variations in the parameters as a function of lowest energy fit; therefore, for all the data presented here only the quadratic coefficient was fitted. Since the best-fit neutrino mass was zero, a fit which would include the nonphysical region where $\mathrm{m}_{\nu}{ }^{2}<0$ was performed to assure that the bestfit value would be statistically consistent with a zero mass. The minimum $\Xi^{2}$ was found at $m_{\nu}{ }^{2}--1190 \mathrm{ev}^{2}$, or $\mathrm{m}_{\nu}=34.5 \mathrm{i} \mathrm{ev} . \Xi^{2}$ increased by 1.2 between the minimum and $0 \mathrm{eV}$, which indicates that the best-fit value for the mass is about $1.1 \sigma$ away. Clearly, this measurement is 


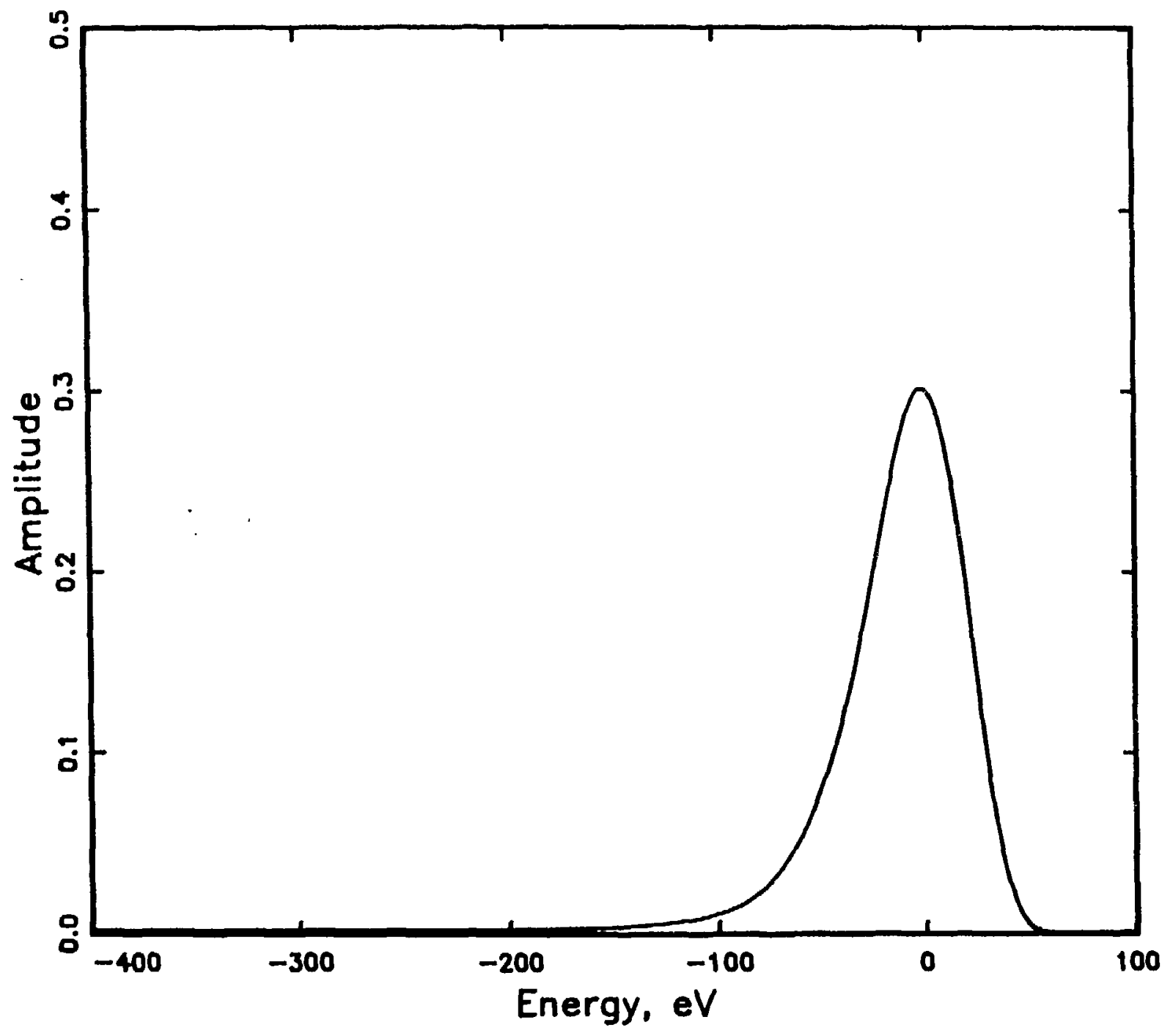

Fig. 6.3. Total experimental resolution function for TBD3. This funition includes the spectrometer resolution, the window width, and the ellelgy loss spectrun. 
Table 6-2:

\section{Data Fits}

\begin{tabular}{lccccccc} 
Data Set & $\begin{array}{c}\text { Mass } \\
\mathrm{eV}\end{array}$ & $\begin{array}{c}\text { Endpoint } \\
\mathrm{eV}\end{array}$ & $\begin{array}{c}\text { BKGD } \\
\mathrm{eV}^{-1} \mathrm{~s}^{-1} \\
\times 10^{4}\end{array}$ & $\begin{array}{c}\text { Amp. } \\
\times 10^{2}\end{array}$ & $\begin{array}{c}\text { Quad. } \\
\mathrm{eV}^{-2} \\
\times 10^{8}\end{array}$ & $\Xi^{2}$ & DOF \\
\hline TBD3 & 0.0 & 18587.4 & 2.43 & 12.6 & -1.18 & 273.2 & 249 \\
TBD3 & $34.5 i$ & 18584.8 & 2.37 & 12.7 & -1.28 & 272.0 & 249 \\
TBD4-1 & 0.0 & 18586.3 & 1.96 & 8.81 & -1.94 & 211.0 & 245 \\
TBD4-1 & 38.9 & 18589.4 & 2.05 & 8.75 & -1.83 & 209.9 & 245
\end{tabular}




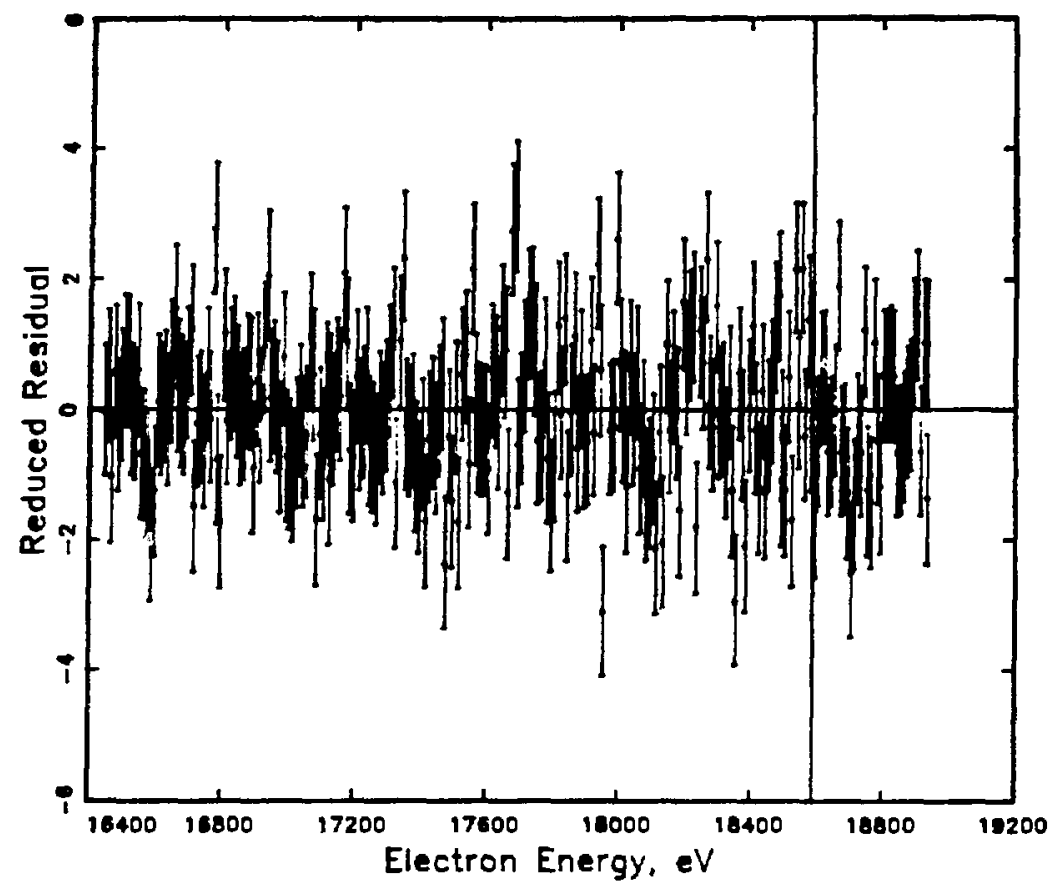

F1g. 6.4. Reduced residuals for $T B D 3, m_{\nu}-0$. The values of the reduced residuals are the values for $\Xi^{2}$ at each point, with error bars of length 1.

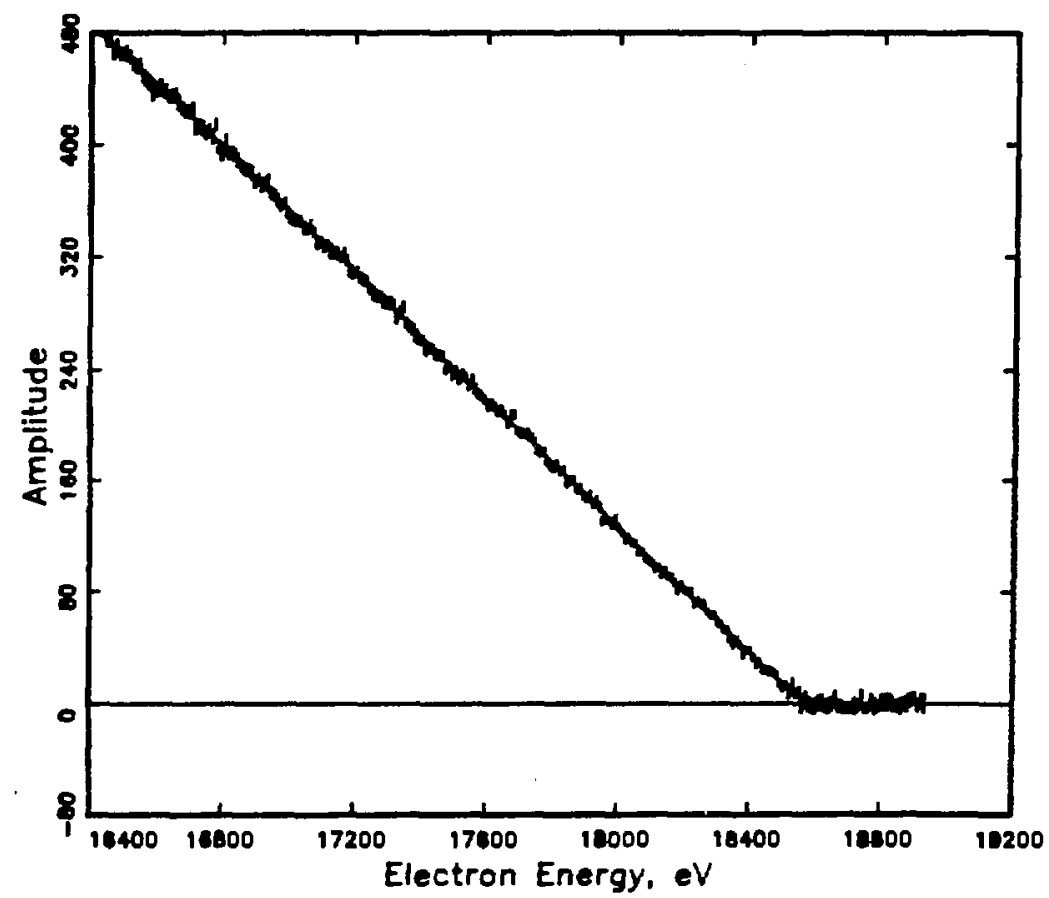

Fig. 6.5. Kurie plot of TBD3, with fit for $m_{\nu}-0$. 
consistent with zero neutrino mass. A $\Xi^{2}$ plot for real values of the neutrino mass is showr in Fig. 6.6 .

A search for systematic errors not readily apparent in the data was performed by fitting progressively less data, as described in Chapt. 5. As can be seen from Table 6-3, no systematic variations in any parameter were observed. This observation provides good evidence that the model used in fitting the data was adequate. The curvature, covariance, and correlation matrices at the minimum of this fit are shown in Table 6-4.

Considering the increase of $\Xi^{2}$ from its value at $m_{\nu}=0$, this measurement yields a $2 \sigma$ upper limit of about $33.5 \mathrm{eV}$. The effect of systematic errors was determined by observing their effect on the bestfit $m_{\nu}{ }^{2}$ of MOCxup data files with similar statistics, and choosing the largest effect seen in $\mathrm{m}_{\nu}{ }^{2}$. This procedure was necessary because the fitting algorithm fits imaginary neutrino masses only with great difficulty, so the fitted mass is good to only about $.2 \mathrm{ev}$, an error which can lead to substantial errors in the determination of the effects of systematic errors. The error in the width of the resolution function yields a $\Delta \mathrm{m}_{\nu}{ }^{2}$ of $70 \mathrm{ev}^{2}$, and the error in the scattering produces a $\Delta \mathrm{m}_{\nu}{ }^{2}$ of $50 \mathrm{ev}^{2}$. Adding these in quadrature and doubling the result to get the $2 \sigma$ limit, the $2 \sigma$ upper limit for this data set becomes $36 \mathrm{eV}$.

Data Set TBD4-1:

The second data set considered here was obtained between March 2 and March 4, 1986. The data reduction was easier for this set than it was for TBD3, since the source pressure was kept fairly constant during 


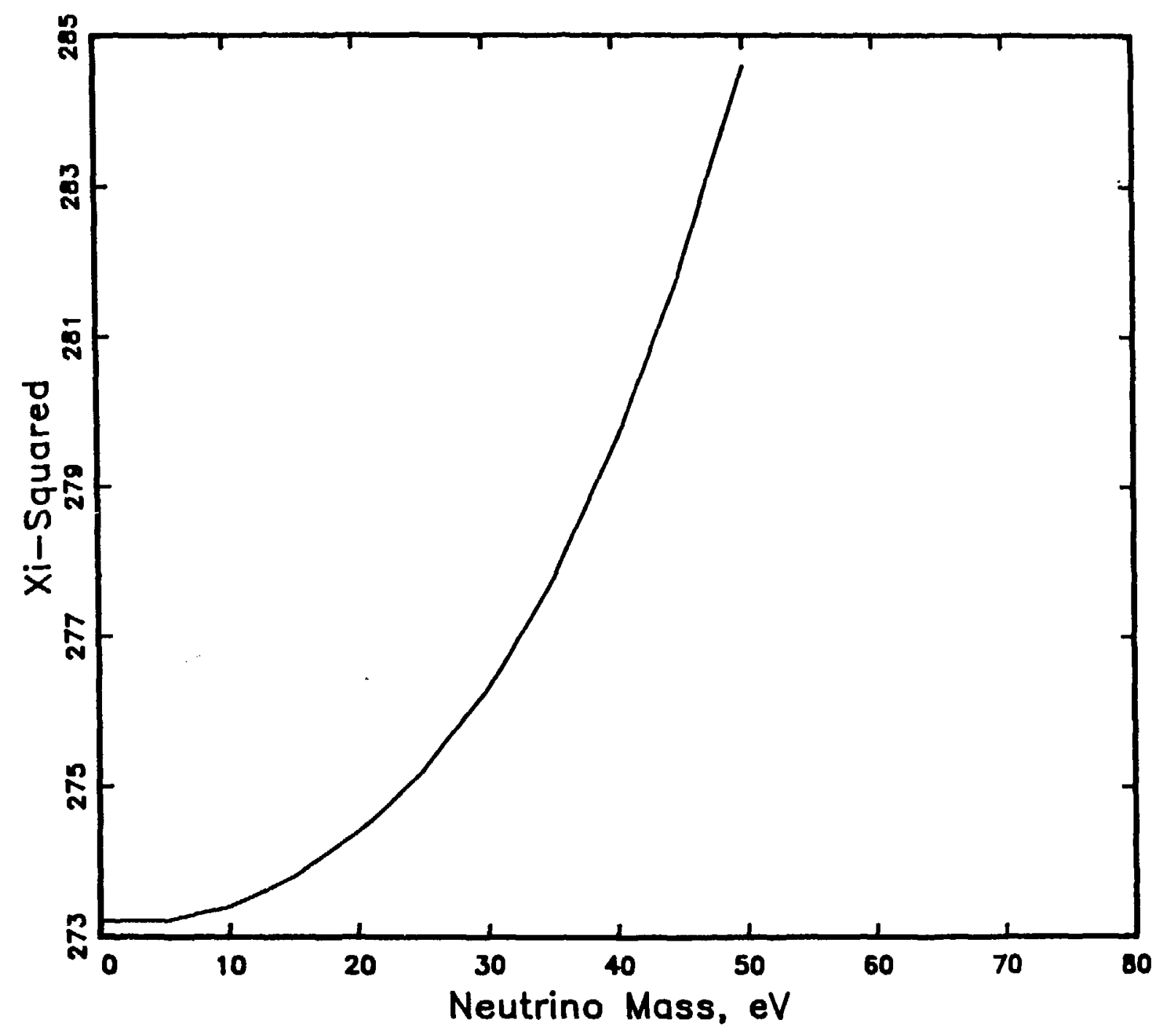

F1g. 6.6. $\Xi^{2}$ plot for TBD3. Only the $\Xi^{2}$ for real values of the neutrino mass are shown. 
Table 6-3:

Systematic Variation Checks

Data Set: TBD3

\begin{tabular}{ccrrrrr}
$\begin{array}{c}\text { Min. Energy } \\
\mathrm{eV}\end{array}$ & $\begin{array}{c}\text { Mass } \\
\mathrm{eV}\end{array}$ & $\begin{array}{c}\text { Endpt. } \\
\mathrm{eV}\end{array}$ & $\begin{array}{c}\text { Amp } \\
\begin{array}{c}\text { BKGD } \\
\mathrm{eV}^{-1} \mathrm{~s}^{-1} \\
\times 10^{4}\end{array}\end{array}$ & $\Xi^{2}$ & DOF \\
\hline 16445 & $35.0 i$ & 18584.7 & .1271 & 2.37 & 272.0 & 250 \\
16645 & $36.4 i$ & 84.3 & .1272 & 2.37 & 261.1 & 229 \\
16845 & $33.1 i$ & 85.1 & .1269 & 2.37 & 240.1 & 209 \\
17045 & $32.3 i$ & 85.4 & .1268 & 2.37 & 229.7 & 189 \\
17245 & $31.3 i$ & 85.5 & .1268 & 2.37 & 215.9 & 168 \\
17445 & $36.8 i$ & 84.1 & .1275 & 2.37 & 198.8 & 148 \\
17645 & $50.0 i$ & 79.3 & .1299 & 2.37 & 175.3 & 128 \\
17845 & $39.9 i$ & 83.3 & .1276 & 2.37 & 144.2 & 108 \\
18045 & $36.3 i$ & 85.0 & .1262 & 2.37 & 114.0 & 89 \\
18245 & $48.6 i$ & 76.0 & .1367 & 2.37 & 85.1 & 69
\end{tabular}

Data set: TBD4-1

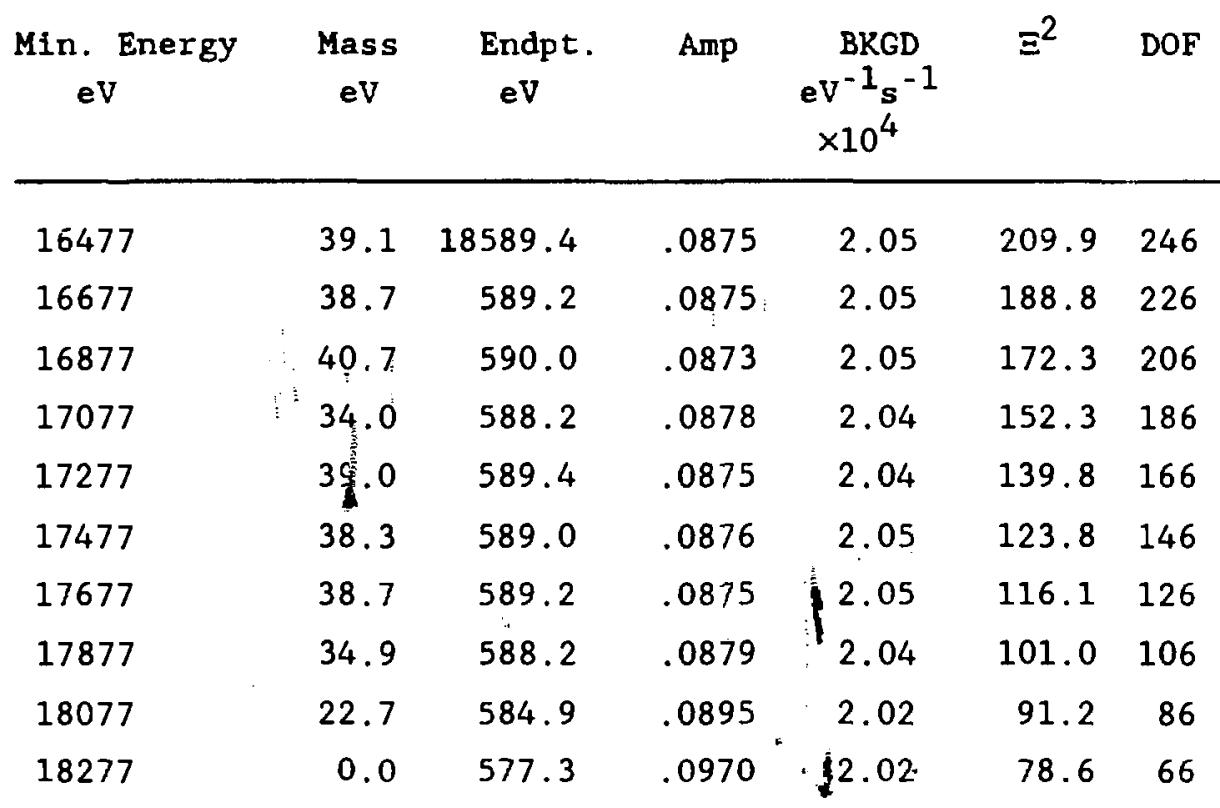


Table 6-4:

Curvature, Covariance, and Correlation Matrices

Data Set TBD3:

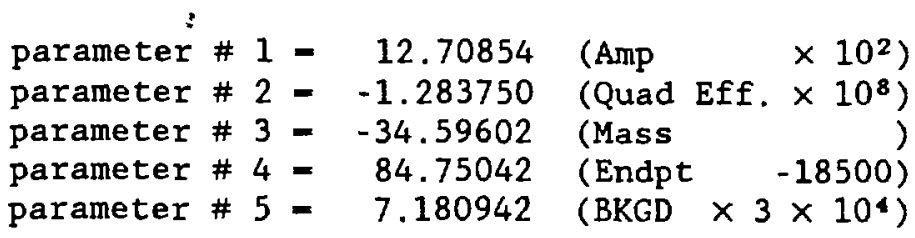

Curvature Matrix:

$\begin{array}{lccccc} & \text { Amp } & \text { Quad } & \text { Mass } & \text { Endpt } & \text { BKGD } \\ \text { Amp } & 2380.072 & 696.1289 & -1.209932 & 52.60359 & 3.310593 \\ \text { Quad } & 696.1289 & 270.0240 & -0.1437998 & 11.42744 & 0.3868461 \\ \text { Mass } & -1.209932 & -0.1437998 & 0.1189086 .-01 & -0.8446690 .-01 & -0.2991164 \text { - } 01 \\ \text { Endpt } & 52.60359 & 11.42744 & -0.8446690-01 & 1.994448 & 0.2453101 \\ \text { BKGD } & 3.310593 & 0.3868461 & -0.29911640-01 & 0.2453101 & 2.009711\end{array}$

Covariance Matrix:

$\begin{array}{lccccc} & \text { Amp } & \text { Quad } & \text { Mass } & \text { Endpt } & \text { BKGD } \\ \text { Amp } & 0.4673121 \bullet-02 & -0.8654434 \bullet-02 & -0.2159939 & -0.8292208 e-01 & 0.8747654 \mathrm{e}-03 \\ \text { Quad } & -0.8654434 \bullet-02 & 0.2127410 e-01 & 0.1867472 & 0.1144029 & -0.1023420 \mathrm{e}-02 \\ \text { Mass } & -0.2159939 & 0.1867472 & 141.7890 & 10.49035 & 1.249707 \\ \text { Endpt } & -0.8292208 \bullet-01 & 0.1144029 & 10.49035 & 2.481208 & -0.3215232 \mathrm{e}-01 \\ \text { BKGD } & 0.8747654 e-03 & -0.1023420 \bullet-02 & 1.149707 & -0.3215232 \mathrm{e}-01 & 0.5173763\end{array}$

Correlation Matrix:

$\begin{array}{lccccc} & \text { Amp } & \text { Quad } & \text { Mass } & \text { Endpt } & \text { BKGD } \\ \text { Amp } & 1.000000 & -0.8679797 & -0.2653486 & -0.7700787 & 0.1779036 \mathrm{e}-01 \\ \text { Quad } & -0.8679797 & 1.000000 & 0.1075244 & 0.4979434 & -0.9754949 \mathrm{e}-02 \\ \text { Mass } & -0.2653486 & 0.1075244 & 1.000000 & 0.5592900 & 0.1342341 \\ \text { Endpt } & -0.7700787 & 0.4979434 & 0.5592900 & 1.000000 & -0.2837772 \mathrm{e}-01 \\ \text { BKGD } & 0.1779036 \mathrm{e}-01 & -0.9754949 \mathrm{e}-02 & 0.1342341 & -0.2837772 \mathrm{e}-01 & 1.000000\end{array}$


Table 6-4 Continued:

Data Set TBD4-1:

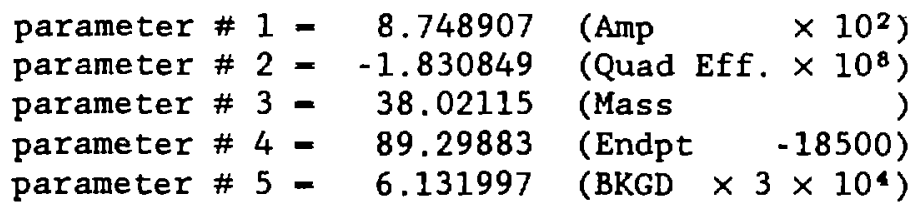

Curvature Matrix:

\begin{tabular}{lccccc} 
& Amp & Quad & \multicolumn{1}{c}{ Mass } & \multicolumn{1}{c}{ Endpt } & \multicolumn{1}{c}{ BKGD } \\
Amp & 4820.149 & 993.2224 & -1.694819 & 71.44900 & 5.951444 \\
Quad & 993.2224 & 268.1780 & -0.1534983 & 11.18045 & 0.5490045 \\
Mass & -1.694819 & -0.1534983 & $0.7456113 .-01$ & -0.1783024 & $-0.7115168 \mathrm{e}-01$ \\
Endpt & 71.44900 & 11.18045 & -0.1783024 & 1.694945 & 0.3129463 \\
BKGD & 5.951444 & 0.5490045 & $-0.7115168 \bullet-01$ & 0.3129463 & 3.052108
\end{tabular}

Covariance Matrix:

$\begin{array}{llcccc} & \text { Amp } & \text { Quad } & \text { Mass } & \text { Endpt } & \text { BKGD } \\ \text { Amp } & 0.4566528-02 & -0.1078359 e-01 & -0.2773530 & -0.1509198 & 0.2043990 e-02 \\ \text { Quad } & -0.1078359-01 & 0.3116402-01 & 0.5515839 & 0.3076317 & -0.3262445 e-02 \\ \text { Mass } & -0.2773530 & 0.5515839 & 36.79808 & 11.90972 & 0.7829701 e-01 \\ \text { Endpt } & -0.1509198 & 0.3076317 & 11.90972 & 6.197450 & -0.1188598 \\ \text { BKGD } & 0.2043990-02 & -0.3262445 e-02 & 0.7829701-01 & -0.1188598 & 0.3382561\end{array}$

Correlation Matrix:

$\begin{array}{lccccc} & \text { Amp } & \text { Quad } & \text { Mass } & \text { Endpt } & \text { BKGD } \\ \text { Amp } & 1.000000 & -0.9039485 & -0.6765926 & -0.8971121 & 0.5200712 \mathrm{e}-01 \\ \text { Quad } & -0.9039485 & 1.000000 & 0.5150772 & 0.6999998 & -0.3177559 \mathrm{e}-01 \\ \text { Mass } & -0.6765926 & 0.5150772 & 1.000000 & 0.7866466 & 0.2219268 \mathrm{e}-01 \\ \text { Endpt } & -0.8971121 & 0.6999998 & 0.7886466 & 1.000000 & -0.8209292 \mathrm{e}-01 \\ \text { BKGD } & 0.5200712 \mathrm{e}-01 & -0.3177559 \mathrm{e}-01 & 0.2219268 \mathrm{e}-01 & -0.8209292 \mathrm{e}-01 & 1.000000\end{array}$


the run. Instead of performing a point-to-point average of the beta monitor ratio $R_{\beta}$, a constant ratio was assumed. There were some small long term variations, but the additional error introduced by these variations was less than the error which would have resulted from a point-to-point average. The normalized data is shown in Fig. 6.7.

For this data set, krypton data was obtained before and after the run. Both scans were taken with the krypton in recirculation mode. The fits to the two peaks are shown in Fig. 6.8 and in Table 6-1. The two peaks are in statistical agreement, with an average skewness of zero, a result which is not surprising since the spectrometer had been tuned to provide the best resolution possible. The width used in the data analysis was the average of the fitted widths for the two scans. The error in this width was calculated in a manner analogous to that of TBD3, but an additional term was introduced resulting from the correction applied for the background pedestal. The total error in the width was $.6 \mathrm{eV}$.

The source pressure for this run was the same as for the second half of TBD3, so the energy loss spectrum and the error in that spectrum were the same. The total resolution function for this data set is shown in Fig. 6.9. It has a FWHM of about $41 \mathrm{eV}$.

The best-fit parameters to this data set are shown in Table 6-2. For this data set, the best-fit neutrino mass was $38.9 \mathrm{eV}$. This value for neutrino mass is in statistical agreement with zero, $\Xi^{2}$ increasing by 1.1 between the minimum and zero neutrino mass, indicating that the value is 1.050 away from zero. The count rate for this data set was lower than for TBD3, a result of the offset introduced into the 


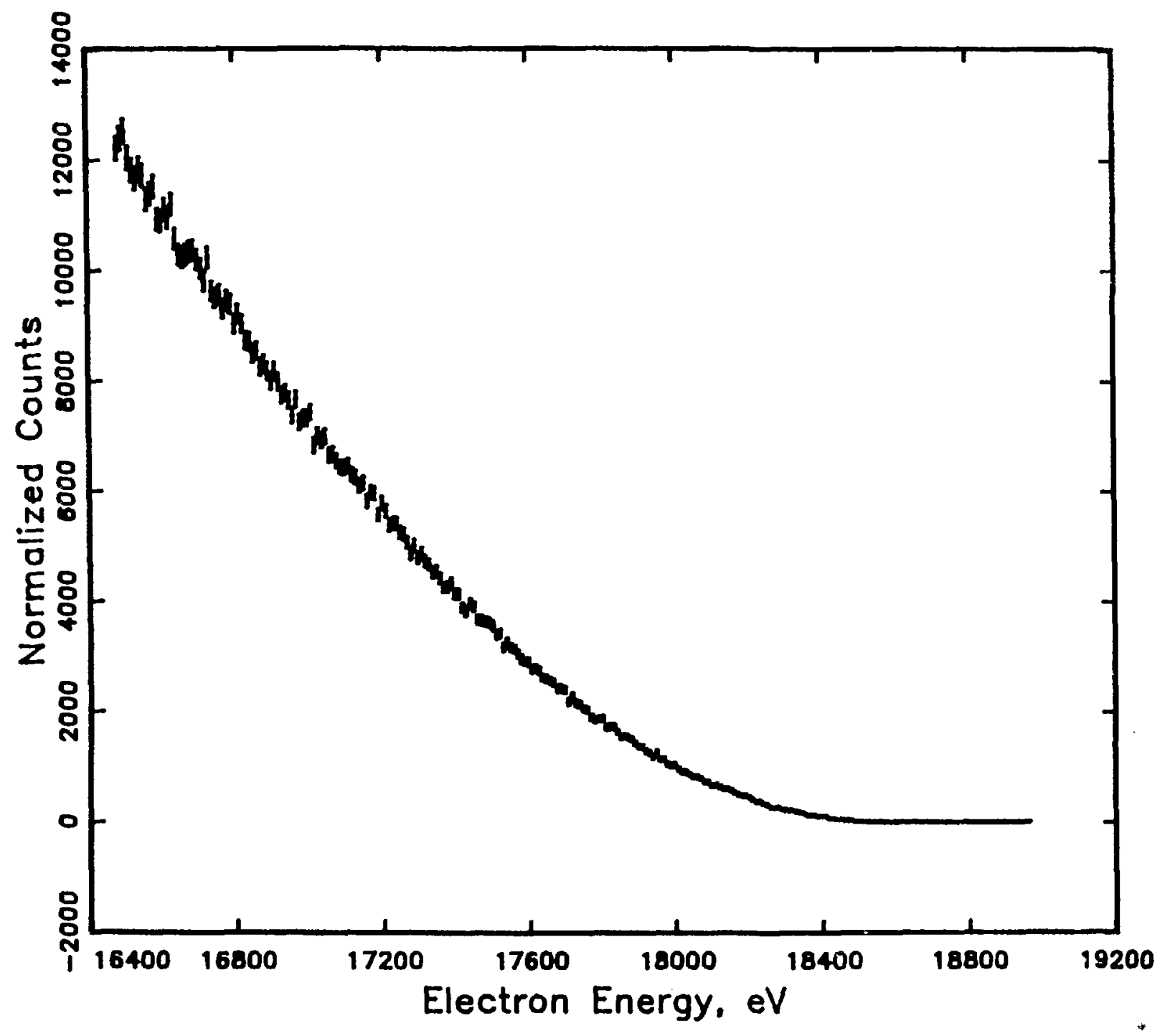

F1g. 6.7. Normalized data for TBD4-1. 

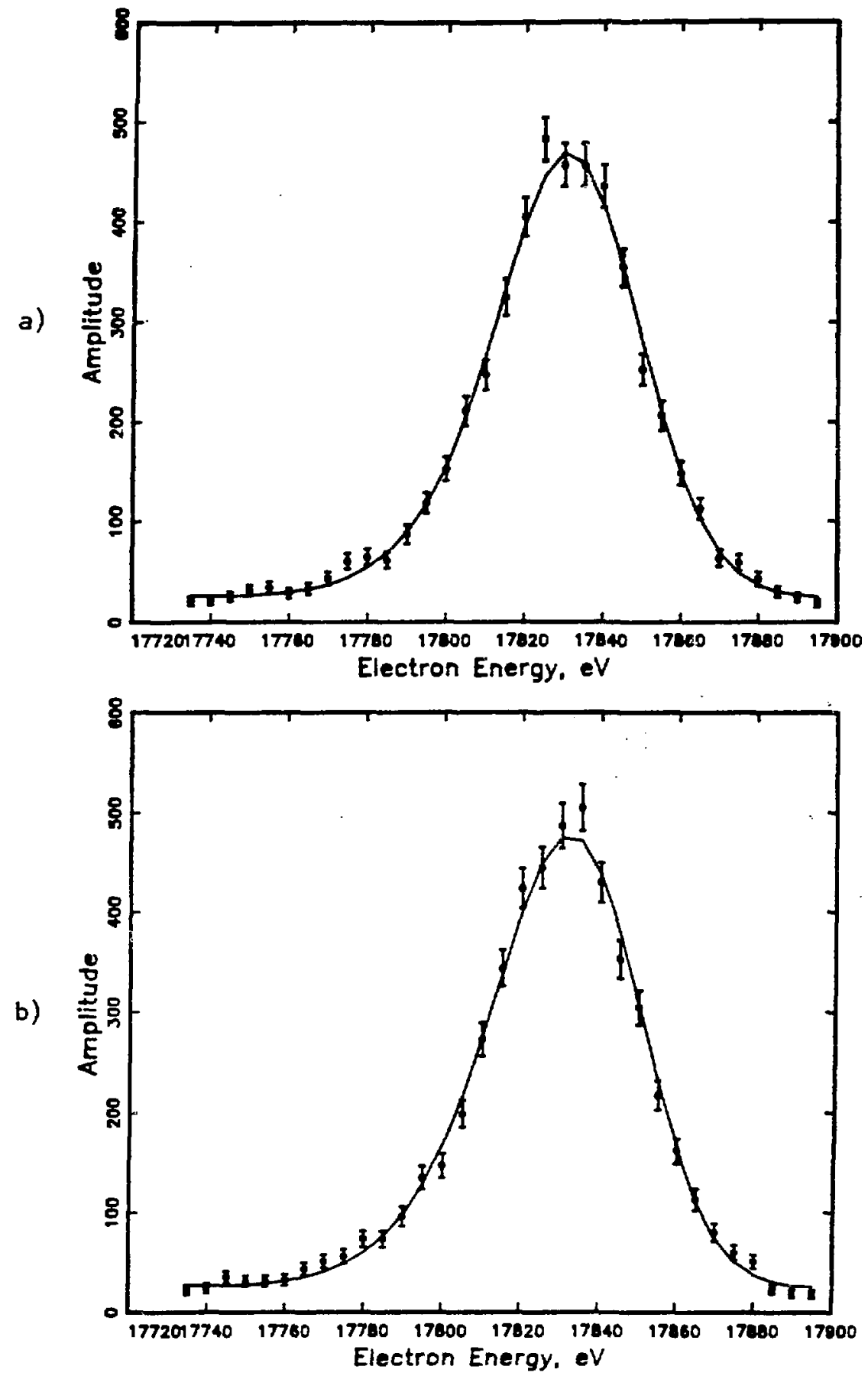

Fig. 6.8. Fits to the krypton peaks for TBD4-1. a) Krypton run taken before data, b) Krypton run taken after data. 


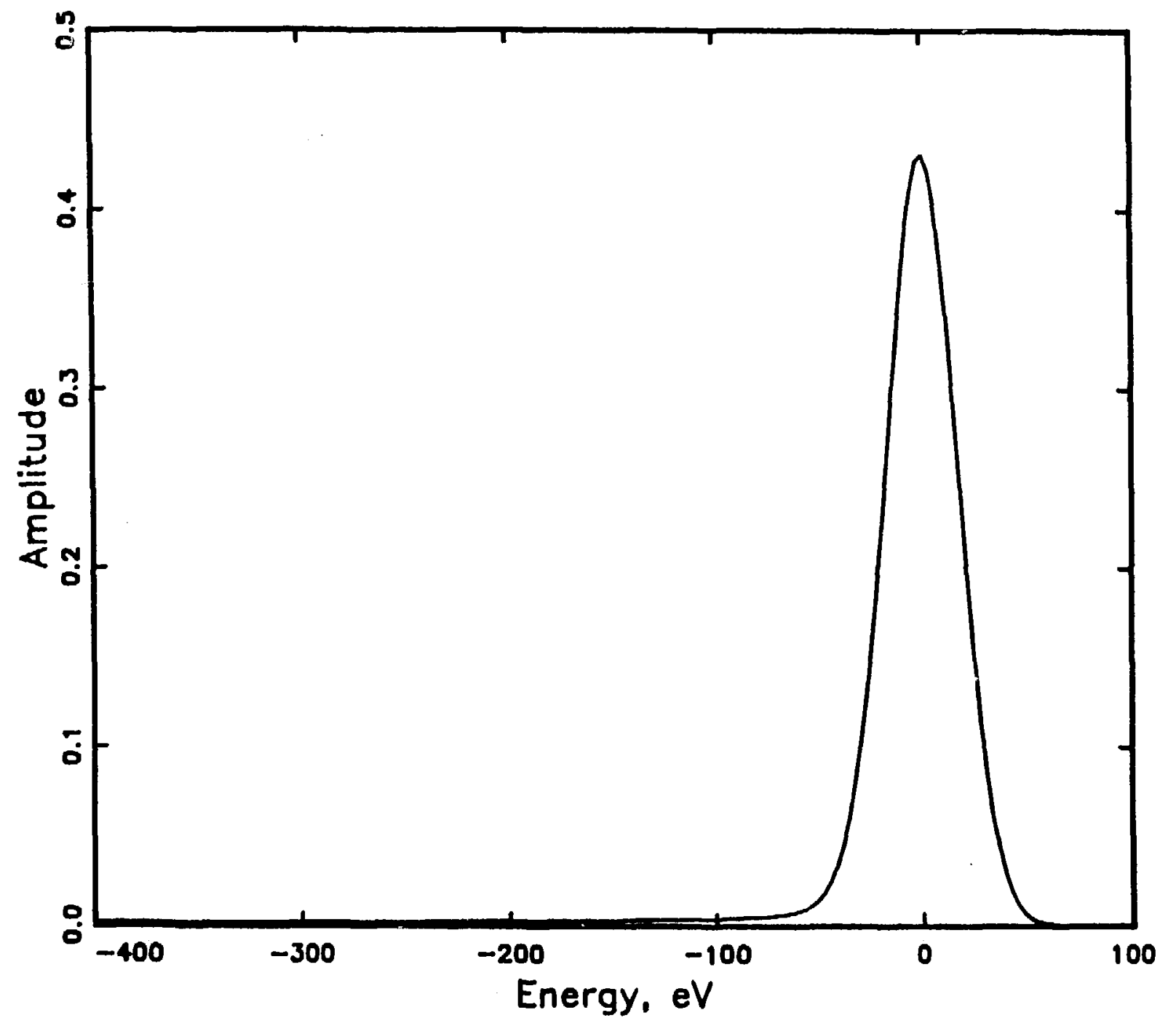

F18. 6.9. Total experinental resolution function for TBD4-1. 
spectrometer compensation field to improve the resolution. The statistical accuracy was therefore somewhat worse than for TBD3. The residuals plot for zero neutrino mass is shown in Fig. 6.10, and the Kurie plot of the data and this fit are shown in Fig. 6.11.

As in TBD3, a check for systematic errors was undertaken by fitting progressively less data. As can be seen in Table 6-3, there was no systematic variation of any parameter. The curvature, covariance, and correlation matrices are shown in Table 6-4.

The $\Xi^{2}$ plot for real values of the neutrino mass is shown in Fig. 6.12. The $2 \sigma$ upper limit which is derived from the increase in $\Xi^{2}$ is about $68 \mathrm{eV}$. The systematic error in the resolution function yields a $\Delta \mathrm{m}_{\nu}{ }^{2}$ of $60 \mathrm{eV}^{2}$ and the systematic error in the energy loss a $\Delta \mathrm{m}_{\nu}{ }^{2}$ of 60 $\mathrm{eV}^{2}$. This yields a $2 \sigma$ upper limit of $69 \mathrm{eV}$.

Combining the Data Sets

The two measurements are in agreement with each other, as long as they both come from a true neutrino mass near zero. The probability of obtaining two measurements farther apart than these two, given a true neutrino mass of zero, is about 148. Further evidence that the data sets are compatible comes from a comparison of the fitted endpoints for zero neutrino mass. These endpoints differ by $1.1 \mathrm{eV}$, compared to the error in each of about $2 \mathrm{eV}$. Therefore, it is likely that the measurements come from the same parent distribution and the $\Xi^{2}$ for both can be added. This procedure results in the $\Xi^{2}$ plot shown in Fig. 6.13. The minimum in this curve is slightly below zero, but certainly consistent with it. The statistical $2 \sigma$ upper limit derived from both 


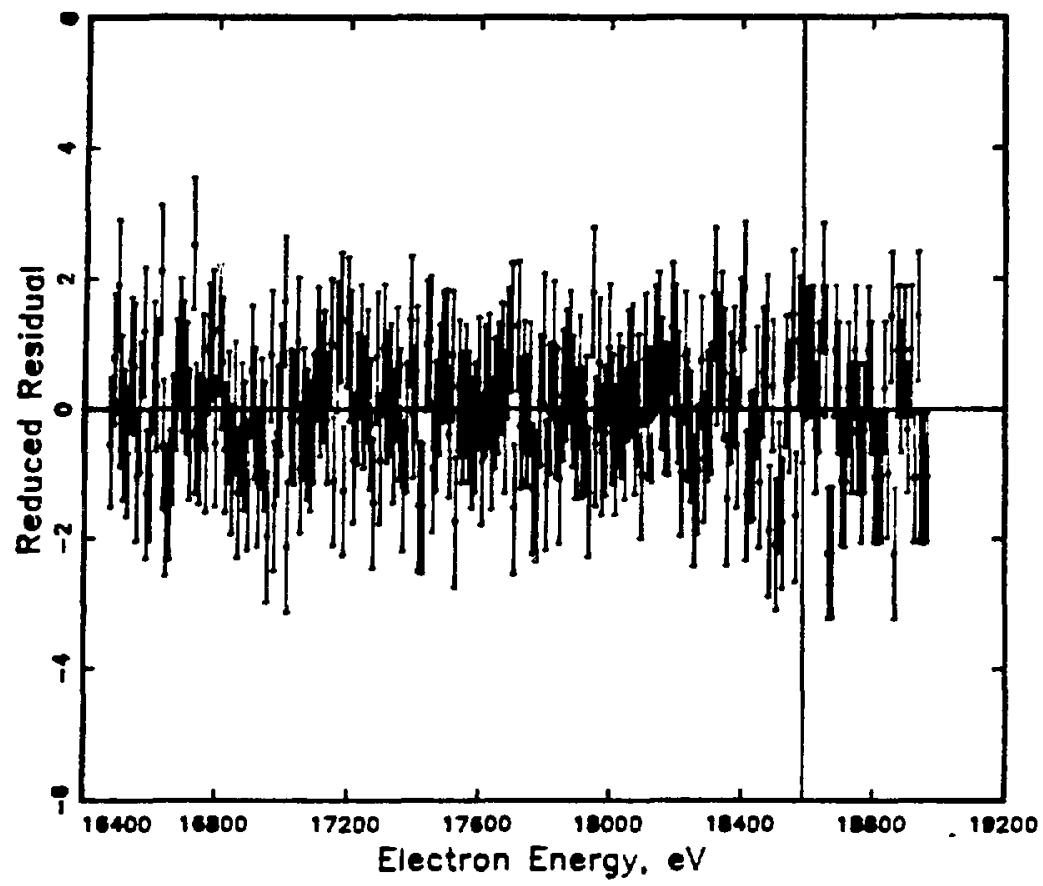

Fig. 6.10. Reduced residuals plot for TBD4-1, $m_{\nu}=0$.

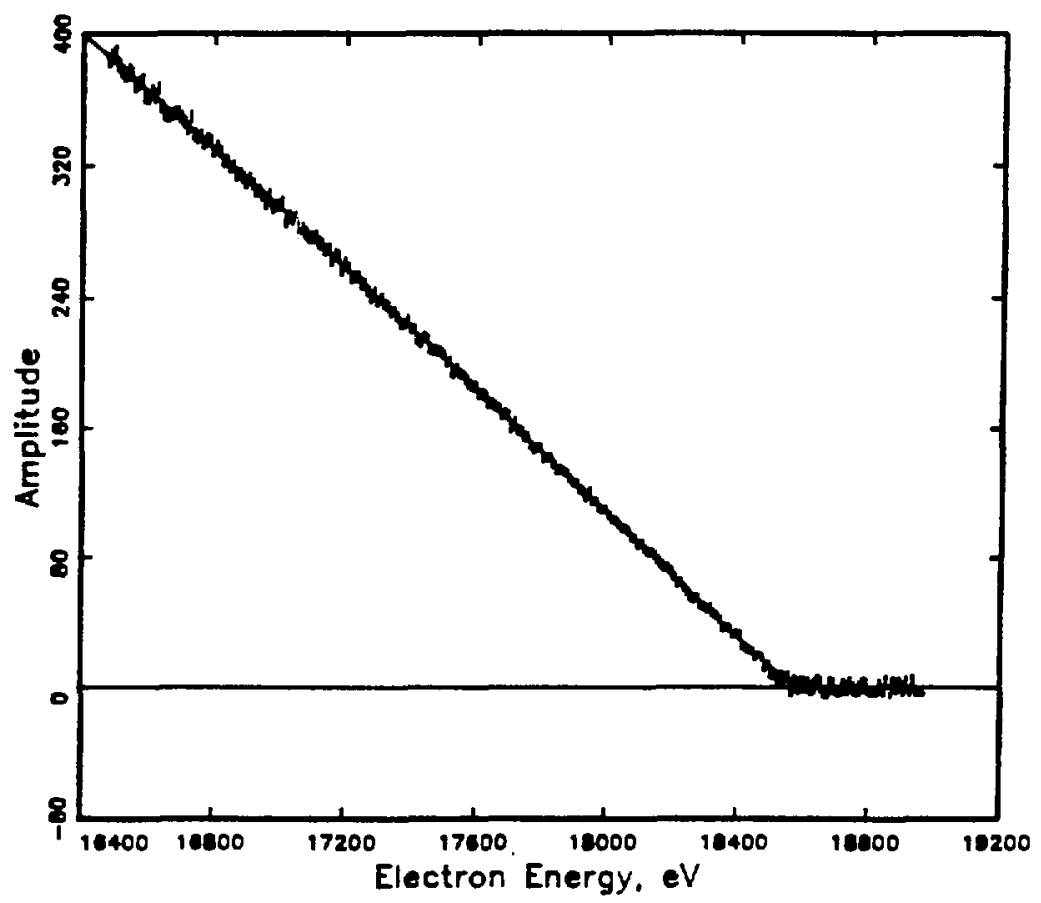

Fig. 6.11. Kurie plot of TBD4-1, with fit to $m_{\nu}=0$. 


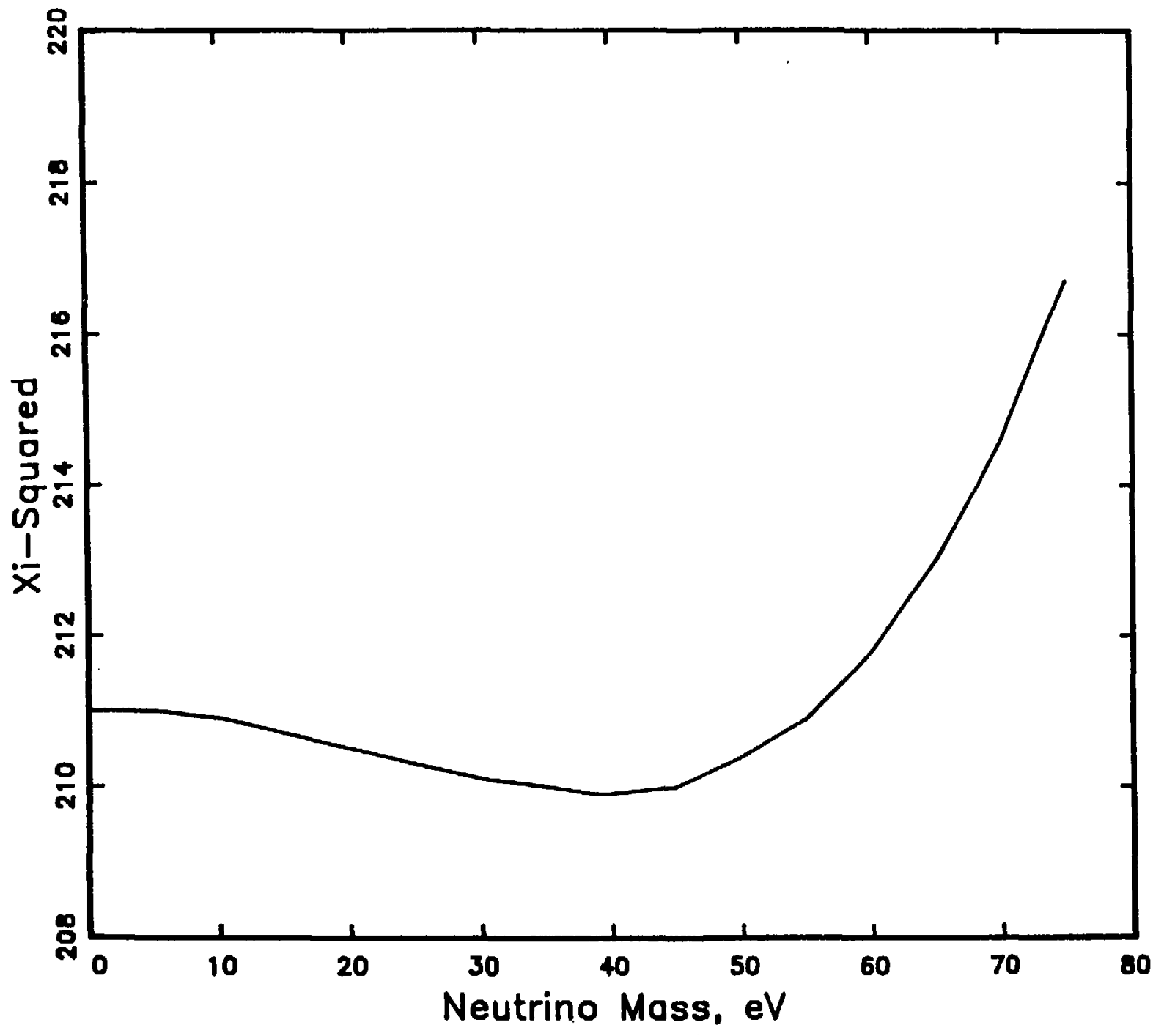

F18. 6.12. $\Xi^{2}$ plot for TBD4-1, real values of neutrino mass. 


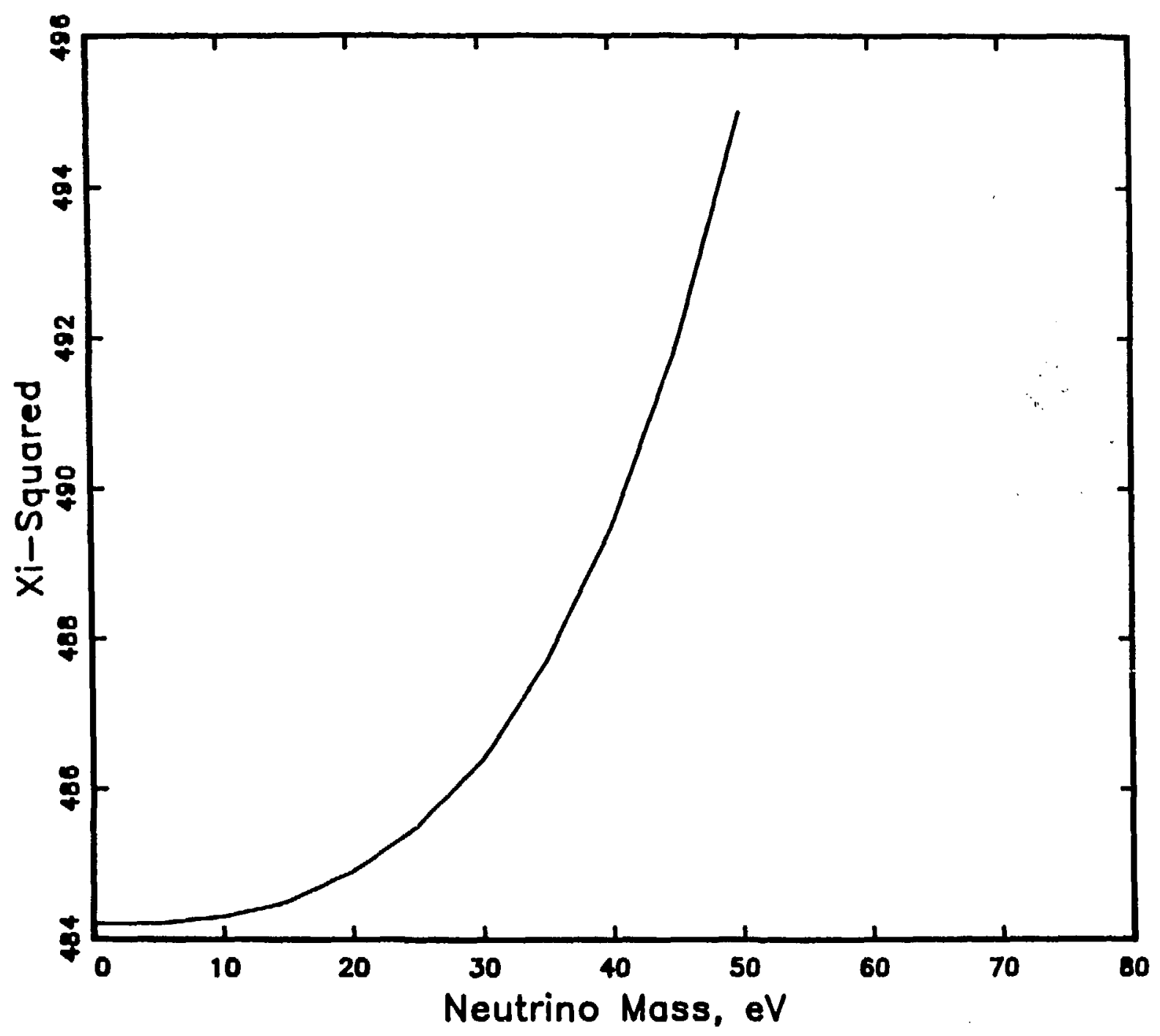

F1g. 6.13. $\Xi^{2}$ plot for both sets of data combined. 
data sets is $37 \mathrm{eV}$, with the systematic error (which is now added coherently for the error in the energy loss, but incoherently for the error in the resolution functions) increasing this to $41 \mathrm{eV}$. The 958 confidence level upper limit is given by the amount that $\Xi^{2}$ must increase from its minimum in order to have 958 of the probability below the value set for the mass. If $\Xi^{2}$ is an unbiased estimator, which it appears to be from data presented in Chapt. 5, this value falls where $\Xi^{2}$ increases by 2.71. This would give a 958 C.I. upper limit on the neutrino mass of $36 \mathrm{eV}$, including the effects of the systematic errors added in at their $95 \%$ C.I. upper limit.

Any statement about the value of the endpoint depends upon an assumption about the value of the neutrino mass. In addition, the endpoint is more sensitive than the neutrino mass to errors in the energy spectrum of the scattered electrons. However, since the endpoints from the two data sets agree for $m_{\nu}=0$, some estimate of the endpoint and its error can be made. The change in the $m_{\nu}=0$ endpoint between data fitted with all of the energy loss going into single scatters, assuming no trapping, and the endpoint for data fitted with the scattering spectrum actually used is about $2 \mathrm{eV}$, averaged between the two data sets. This can be taken as an upper limit to the size of the effect that might be seen from systematic errors in the energy loss spectrum. The error in the relative calibrations between runs can be derived from the errors in the positions of the krypton peaks, about $2 \mathrm{eV}$, and another $1 \mathrm{eV}$ systematic error from variations in the calibration voltage. All these systematic errors yield a total systematic error of $3 \mathrm{eV}$ in the endpoint energy. The statistical error 
in the endpoint energies of each run are about $2.5 \mathrm{eV}$, so the statistical error in the mean is $1.3 \mathrm{eV}$. Therefore, the endpoint energy, corrected for the krypton calibrations, is about $18586.5 \pm 1.3 \pm 3 \mathrm{eV}$. This estimation is, admittedly, a bit crude, but a more detafled analysis is not justiried since the energy of the $\mathrm{Kr}$ line is known only to about $20 \mathrm{eV}$. Therefore, the absolute endpoint energy cannot be known to better than that accuracy. This endpoint energy gives a ${ }^{3} \mathrm{H}-3^{3} \mathrm{He}$ mass difference of $18605.2 \pm 20 \mathrm{eV}$, in good agreement with ion cyclotron resonance measurements [Lip85]. 


\section{Chapter 7:}

\section{Conclusion}

\section{Results}

This experiment has been able to set an upper limit of $36 \mathrm{eV}$ on the electron antineutrino mass, using two scans of the tritium beta decay spectrum near the endpoint. A measurement of the endpoint of the spectrum, which is dependent upon the value of the energy of the $k$ conversion line in krypton, yields $18586.5 \pm 1.3 \pm 3 \mathrm{eV}$ for $\mathrm{m}_{\nu}=0$ and a conversion Iine energy of exactly $17835 \mathrm{eV}$. This result does not contradict the ITEP result of $30 \mathrm{eV}$ for the mass, but it does exclude the upper range of the ITEP result and makes a $35 \mathrm{eV}$ neutrino mass unlikely. This is the first measurement of the beta spectrum of gaseous tritium, and is therefore significant in showing the potential of this kind of experiment. Currently, the limit on the neutrino mass is dominated by statistical errors. The systematic uncertainties associated with this upper limit are considerably less than those which would be encountered in an experiment using a solid source, because the energy loss effects are less significant and the final state distribution is known. Because of the knowledge of the molecular final states, this measurement represents the best model-independent upper limit on the neutrino mass, and the first such measurement for many years. The analysis of this data is also the first to use a maximum- 
likelihood estimator which preserves the area of the spectrum, a possible source of considerable systematic error in the analyses of other experiments.

\section{Future Directions for the Experiment}

The data obtained so far in this experiment indicate that this technique has great potential for reducing the limit on the neutrino mass. However, there are several improvements to this experiment which are necessary to obtain the best possible upper limit on the neutrino mass, expected to be on the order of $10 \mathrm{eV}$. The upper limit which has been set in this work has been obtained by making conservative assumptions about many parameters, including the energy loss in the source and the choice of a quadratic efficiency term. This limit could be improved immediately by a more precise understanding of the actual energy loss in the source and by an a priori justification for the use of the a quadratic instead of a linear efficiency term. Over a longer term, the count rate must be increased while the background is reduced, and the experimental resolution must be improved. There are also possible improvements to the beta monitor which may help the experiment be more sensitive to the neutrino mass.

Because the direction of the curvature in the spectrum is negative, a fit with a linear term always gives a lower neutrino mass than a fit to a quadratic term. The magnitude of this effect depends on the details of the spectrum and the resolution function, but it can be significant. The size of the efficiency term is fairly small for the data presented here, decreasing the normalized data by about 108 at the 
lowest energies taken. The quadratic term was used because it is less covariant with the neutrino mass than is the linear term and because the systematic variations with energy observed using a linear term indicated that it was not as good a description of the shape of the data as the quadratic term was. However, it is impossible to distinguish which of the two terms is the correct one based on the $\Xi^{2}$ of each because the statistics for these spectra are not good enough. Some things can be done to improve the knowledge of the efficiency variations. The efficiency of the beta monitor as a function of energy can be mapped out over a large energy range, which would make it easier to distinguish between the quadratic and linear efficiency terms. The efficiency variations can be observed at different spectrometer settings, which allows the variation of the efficiency with accelerating voltage to be observed for different electron energies. Lower initial electron energies can be detected by raising the maximum voltage on the source tube, which once again will allow the difference between the quadratic and linear terms to be seen more easily. Finally, the counting rate can be increased, which will provide better statistical accuracy and therefore a better determination of the shape of the efficiency variation.

The energy loss in the source can most easily be reduced by implementing the control system for the gradient in the field of the solenoid to eliminate trapped particles and to reduce the average path length. More detailed ray-tracing calculations which will improve the estimates of the total scattering probability and the trapping energy loss can be undertaken. Finally, the energy loss can be studied by 
mixing krypton with various pressures of hydrogen and measuring the shape of the peak. This measurement has not been possible yet because of leaks in the source, which cause the pressure of gas recirculated with the krypton to increase fairly quickly, contaminated with an unknown mixture of gases.

Probably the most important improvement in the experiment will be the increase in the count rate. The originally anticipated count rate is about 10 times the count rate observed so far. Much of this problem is a result of not having all the features implemented yet. One problem is the low tritium pressure necessitated by the high-voltage discharge in the source. When this problem is corrected by the replacement of a defective sapphire piece, the source pressure can be increased by a factor of at least two. In addition, a new detector, with a better position resolution and a longer window, will improve the count rate by about a factor of three without sacrificing the experimental resolution. Part of the problem is the acceptance of the spectrometer, which has been reduced somewhat by the offset introduced into the compensation fields in order to improve the resolution. The geometrical errors responsible for this problem will be corrected, and the resolution will presumably improve along with the count rate. But further improvements in the count rate will depend on improvements in the extraction of betas from the source. The ray-tracing codes have been improved and can be used to investigate the effects of various corrections to the extraction field. It is quite likely that improvements in the extraction field will improve the count rate by another factor of two. All these improvements would add a factor of at least 10 in the count rate. 
The background rate will be reduced by the improved position resolution of a new detector, and by another feature of it as we11. The new detector, which will have multiple cathode wires, will be able to discriminate between beta events, which enter the detector radially and are stopped in a short distance, and cosmic-ray events, which can enter the detector in any direction and usually pass through. These improvements may decrease the background by a factor of two.

The spectrometer resolution will be improved by the correction of geometrical errors in the spectrometer, and by further improvements in the homogeneity of the ambient field. The resolution will quite likely be improved to about $30 \mathrm{eV}$ FWHM.

Finally, the beta monitor can be improved to generate smaller errors in the data points at energies far below the endpoint, where the error in the beta monitor ratio is currently the dominant component of the error. The background in the beta monitor will be improved by installing an interlock which will allow the detector to be isolated from the rest of the vacuum system when the tritium pressure is high, and thus minimize the tritium contamination of its surface. The electronics will be improved to reduce the temperature sensitivity of the gain and to improve the stability of the linear gate stretcher which is used as a lower level discriminator. This will reduce the systematic variations in the beta monitor ratio.

Once these improvements are in place, a great deal more data with molecular tritium will be obtained. As a check of the result obtained with molecular tritium, spectra will eventually be obtained from a source of atomic tritium. It seems probable that the eventual 
sensitivity of the experiment to neutrino mass will be around $10 \mathrm{eV}$, and that it will prove to be a valuable and reliable measurement. The original purpose of this experiment was to provide a believable limit on the neutrino mass. Thus far, it has lived up to its promise. 


\section{References:}

Alt85 T. Altzitzoglou, R. Kouzes, F. Loeser, M. Lowry, and R. Naumann, Phys. Rev. C31, 360 (1985)

Alv39a L.W. Alvarez and R. Cornog, Phys. Rev. 56, 613 (1939)

Alv40a L.W. Alvarez and R. Cornog, Phys. Rev. 57, 248a (19:40)

Alv40b L.W. Alvarez and R. Cornog, Phys. Rev. 58, 197a (1940)

And82 J. Andersen, G. Beyer, G. Charpak, A. de Rujula, B. Elbek, H. Gustafsson, P. Hansen, B. Jonson, P. Knudsen, E. Laegsgaard, J. Pedersen, and H. Ravn, Phys. Lett. 113B, 72 (1982)

Bah84

J. Bahcal1, AIP Conf. Proc. \#126, p.60 (Homestake 1984)

Bak83 S. Baker and R. Cousins, Nucl. Instrum. and Meth. 221, 437 (1983)

BAL01 Balzers Mode1 QMA 140 Quadrupole Analyzer with Mode1 QME 201 Electrometer Amplifier, Balzers AG, Balzers, Liechtenstein

Bam72 W. Bambynek, B. Crasemann, R. Fink, H. Freund, H. Mark, C. Swift, R. Price, P. Venogopala Rao, Rev. Mod. Phys. 44, 716 (1972)

Be183 E. Bellotti, O. Cremonesi, E. Fiorini, C. Liguori, A. Pullia, P. Sverzellati, and L. Zanotti, AIP Conf. Proc. \#96, (Los Alamos 1983)

Ben80 C. Bennett, A. Hallin, P. Springer, and R. Naumann, Princeton Nuclear Physics Proposal, 1980 (unpublished) 
Ben81 C. Bennett, A. Hallin, R. Naumann, P. Springer, and $M$. Withere11, Phys. Lett. 107B, 19 (1981)

Ben84 C. Bennett, Neutrino Mass and Low-Energy Weak Interactions, (Telemark 1984), p. 128 (1984)

Ben85 C. Bennett, A. McDonald, P. Springer, T. Chupp, and M. Tate, Phys. Rev. C31, 197 (1985)

Ber72a K.E. Bergkvist, Nuc1. Phys. B39, 317 (1972)

Ber72b K.E. Bergkvist, Nucl. Phys. B39, 371 (1972)

Ber82 K. Bergkvist, letter to V. Lyubimov, 1982

Ber85a K. Bergkvist, Phys. Lett. 154B, 224 (1985)

Ber85b K. Bergkvist, Phys. Lett. 159B, 408 (1985)

Bet39 H.A. Bethe, F. Hoyle, and R. Peierls, Nature 143, 200 (1939)

Boe84 F. Boehm, Neutrino Mass and Low-Energy Weak Interactions, p.168 (Telemark 1984)

Bor83 S. Boris, A. Golutvin, L. Laptin, V. Lyubimov, V. Nagovizin, E. Novikov, V. Nozik, V. Soloshenko, I. Tichomorov, and E. Tret'yakov, Preprint 1983

Bor 85 S. Boris, A. Golutvin, L. Laptin, V. Lyubimov, V. Nagovizin, E. Novikov, V. Nozik, V. Soloshenko, I. Tichomorov, and E. Tret'yakov, Phys. Lett. 159B, 217 (1985)

Bro41 S.C. Brown, Phys. Rev. $\underline{59}, 954$ (1941)

Bru85 V. Brudanin, T. Vylov, C. Briancon, V. Gorojankin, K. Gromov, A. Marinov, A. Novgorodov, V. Pokrovski, N. Rukhadze, and V. Sidorov, Nucl. Phys. 11, L119 (1985) 
Bya48 W.J. Byatt, F.T. Rogers, and A. Waltner, Phys. Rev. 74, 699 (1948)

Bya49 W.J. Byatt, F.T. Rogers, and A. Waltner, Phys. Rev. 75, 909 (1949)

$\operatorname{Car} 73$

T. Carlson and C. Nestor, Phys. Rev. A8, 2887 (1973)

Cav84

J. Cavaignac, A. Hoummada, D. Koang, B. Vignon, Y. Declais,

H. deKerret, H. Pessard, and J. Thenard, Phys. Lett. 148B, 387 (1984)

CCLO1

Cryogenic Consultants, Ltd., Metrostore Bldg., 231 The Vale, London, England

Cea84

P. Cea, Phys. Lett. 146B, 75 (1984)

Coo50

E.P. Cooper and F.T. Rogers, JrPhys. Rev. 77, 402 (1950)

Cow72

R. Cowsik and J. McClelland, Phys. Rev. Lett. 29, 669 (1972)

Cra66

K. Cranda11, Los Alamos Report LA-3512 (1966)

Cra84

K. Crandall, private communication (1984)

CTI01

CTI Model 21SC Cryocooler, with Model SCW Compressor Unit, CTI-Cryogenics, Kelvin Park, Waltham, MA

Cur48

S.C. Curran, J. Angus, and A.L. Cockroft, Nature 162, 302 (1948)

Cur49a S.C. Curran, J. Angus, and A.L. Cockroft, Phil. Mag. 40, 53 (1949)

Cur49b S.C. Curran, J. Angus, and A.L. Cockroft, Phys. Rev. 76, 853 (1949)

Dar69

R. Daris and C. St-Pierre, Nucl. Phys. A138, 545 (1969) 
Dav81 M. Davis, M. Lecar, C. Pryor, and E. Witten, Astrophys. Journal 250, 423 (1981)

Dus49 S. Dushman, Scientific Foundations of Vacuum Technique, Wiley \& Sons (1949)

Ead71 W. Eadie, D. Drijard, F. James, M. Roos, and B. Sadoulet, Statistical Methods in Experimental Physics, North-Holland (1971)

EDC01 EDC Mode1 501 Series J, Electronic Development Corp., 11 Hamlin St., Boston, MA

EDW01 Edwards Mode1 9M3A Mercury Diffusion Pump, Edwards High Vacuum, Manor Royal, Crawley, Sussex, England

EDW02 Edwards Mode1 244B Mercury Diffusion Pump, Edwards High Vacuum, Manor Royal, Crawley, Sussex, England

Fab79 S.M. Faber and J.S. Gallagher, Ann. Rev. Astron. Astrophys. 17. 135 (1979)

Fer34 E. Fermi, Z. Phys 료, 11 (1934)

For83 A. Forster, H. Kwan, J. Markey, F. Boehm, And H. Hendrickson, AIP Conf. Proc. 114, 199 (Blacksburg 1983)

Fra82 P. Frampton and P. Vogel, Phys. Rev. $\underline{82}, 339$ (1982)

Fri85 J. Friar, private communication (1985)

Fri86 M. Fritschi, E. Holzschuh, W. Kundig, J. Petersen, R. Pixley, and H. Stussi, preprint (1986)

Gei64 J. Geiger, Z. Phys. 181, 413 (1964) 
M. Gell-Mann, P.Ramond, and R. Slansky, Supergravity, eds. P. van Nieuwenhuizen and D.Z. Frredman (North-Holland, Amsterdam, 1979)

Gra49

E.R. Graves and D.I. Meyer, Phys. Rev. 76, 183a (1949)

Gut81

A.H. Guth, Phys. Rev. D23, 347

$\operatorname{Ham} 52$

D.R. Hamilton, W.P. Alford, and L. Gross, Phys. Rev. $\underline{83}, 215$ (1952)

Han49 G.C. Hanna and B. Pontecorvo, Phys. Rev, 75, 983 (1949)

Har82 F.D.A. Hartwick, Astrophys. Journal 255, L91 (1982)

Hax83 W. Haxton and G. Stephenson, Prog. in Part. and Nuc1. Phys. 12, 409 (1983)

Hut 84

P. Hut and S.D.M. White, Nature $\underline{310}, 637$ (1984)

Hyn83 M. Hynes, J. Kelly, B. Peterson, and B. Norum, preprint 1983 IMSO1 Routine GGPON, from the International Mathematical And Statistical Library, 75000 Bellaire Boulevard, Houston, TX.

IMSO2

Routine GGNML, from the International Mathematical And Statistical Library, 75000 Bellaire Boulevard, Houston, TX.

IMSO3

Routine 2XSSQ, from the International Mathematical And Statistical Library, 75000 Bellaire Boulevard, Houston, TX.

Jen49 G.H. Jenks, J.A. Ghormley, and F.H. Sweeton, Phys. Rev. $\underline{75}$, 701 (1949)

Jen50 G.H. Jenks, J.A. Ghormley, and F.H. Sweeton, Phys. Rev. $\underline{80}$, $990(1950)$ 
Jon83 B. Jonson, J. Andersen, G. Beyer, G. Charpak, A. de Rujula, B. Elbek, H. Gustafsson, P. Hansen, P. Knudsen, E. Laegsgaard, J. Pedersen, and H. Ravn, Nucl. Phys. A396, 479c (1983)

Kai83 N. Kaiser, Astrophys. Journal 273, L17 (1983)

Kir83 T. Kirsten, H. Richter, and E. Jessberger, Phys. Rev. Lett. 50,474 (1983)

Koa84 D. Koang, J. Cavaignac, A. Hoummada, B. Vignon, Y. Declais, H. deKerret, H. Pessard, and J. Thenard, Proc. XIth Int. Conf. on Neutrino Physics and Astrophysics, p.104 (1984)

Ko185 W. Kolos, B. Jeziorski, and K. Szalewicz, Phys. Rev. A31, 551 (1985)

Kon35 E.J. Konopinski and G.E. Uhlenbeck, Phys. Rev. 48,7 (1935)

Kon47 E.J. Konopinski, Phys, Rev. 72,518 (1947)

Kra84 L.M. Krauss, Neutrino Mass and Low-Energy Weak Interactions, p. 20 (Telemark 1984)

Kur36 F.N.D. Kurie, J.R. Richardson, and H.C. Paxton, Phys. Rev. 49, 368 (1936)

Lan52 L.M. Langer and R.J.D. Moffat, Phys. Rev. $\underline{88}, 689$ (1952)

LEC01 LeCroy Model 3001 qVt Multichannel Analyzer, LeCroy Research Systems Corp., $700 \mathrm{~s}$. Main St., Spring Valley, NY

Lev53 J. Levinger, Phys. Rev. 90, 11 (1953)

Lew70 V.E. Lewis, Nuc1. Phys. A151, 120 (1970)

Lib39 W.F. Libby and D.D. Lee, Phys. Rev. 55, 245 (1939) 
Lip85 E. Lipmaa, R. Pikver, E. Suurmaa, J. Past, J. Puskar, I. Koppe1, and A. Tammik, Phys. Rev. Lett. 54, 285 (1985)

Liu73

J. Liu, Phys. Rev. A7, 103 (1973)

Lon49

C. Longmire and H. Brown, Phys. Rev. 75, 264 (1949)

Los 85

J. LoSecco, J. Thomas, D. deJongh, B. Yip, J. Gimlett, T. Altzitzoglou, and R. Naumann, preprint 1985

Lyu80a

V. Lyubimov, E. Novikov, V. Nozik, E. Tret'yakov, and V. Kosik, Phys. Lett. 94B, 266 (1980)

Lyu80b V. Lyubimov, E. Novikov, V. Nozik, E. Tret'yakov, V. Kosik, and N. Myasoedov, Zh. Eksp. Teor. Fiz. 11,1158 (1980)

Lyu81 V. Ly:abimov, Soviet Atomic Energy 49, 804, Pub1ished in Soviet Phys. Rev. 4, 2.1 (1982)

Lyu82 V. Lyubimov, letter to J. Simpson, 1982

Lyu86 V. Lyubimov, preprint 1986

Mar85 R. Martin and J. Cohen, Phys. Lett. 110A, 95 (1985)

Moe80 M. Moe and D. Lowenthal, Phys. Rev. C22 2186 (1980)

Mo132 C. Moller, Ann. Physik 14, 568 (1932)

NBS51 Nat'1 Bureau of Standards Applied Mathematics Series \#13 (1951)

Nie41 C.E. Nielsen, Phys. Rev. $\underline{60}$, 160a (1941)

One40a R.D. O'Neal and M. Goldhaber, Phys. Rev, 57, 1086a (1940)

One40b R.D. O'Neal and M. Goldhaber, Phys. Rev. 58, 574 (1940)

One41 R.D. O'Neal, Phys. Rev. 60, 359 (1941) 


\author{
Pe183 R. Peierls, Contemp. Phys. 24, 221 (1983) \\ Per40 G.J. Perlow, Phys. Rev. 58, 218 (1940) \\ Pet86 J. Petersen, talk given at Moriond 1986 \\ Por59 F.T. Porter, Phys. Rev. 115, 450 (1959) \\ Pru48 J.R. Pruett, Phys. Rev. 73, 1219a (1948) \\ Rag83 R. Raghavan, Phys. Rev. Lett. 51, 975 (1983) \\ Rei80 F. Reines, H. Sobel, and E. Pasierb, Phys. Rev. Lett. $\underline{45}$, \\ 1307 (1980) \\ Ron83 M. Roncadelli and D. Wyler, Phys. Lett. 133B, 325 (1983) \\ Ros36 M.E. Rose, Phys. Rev. 49, 727 (1936) \\ Ros 86 S.P. Rosen, talk given at Moriond 1986 \\ Row84 J. Rowley, B. Cleveland, and R. Davis, AIP Conf. Proc. \#126, \\ p.1 (Homestake 1984) \\ Rub83 V.C. Rubin, Science 220, 1339 (1983) \\ Ruj81 A. de Rujula, Nucl. Phys. B188, 414 (1981) \\ SAE01 S.A.E.S. Model GP50W, S.A.E.S. Getters S.p.A., Via Gallarate \\ 215, Milano, Italy \\ Sal69 R.C. Salgo and H.H. Staub, Nucl. Phys. A138, 417 (1969) \\ Sch81 D. Schramm and G. Steigman, Astrophys. Journal 243, 1 (1981) \\ Sim81 J.J. Simpson, Phys. Rev. D23, 649 (1981) \\ Sim82 J. Simpson, letter to V. Lyubimov, 1982
}


S1a49

L. Slack, G.E. Owen, and H. Primakoff, Phys. Rev. 75, 1448 (1949)

Spe 74

Tak84

Tre 75

Tre76

U1s 74

Vui84

Wa182

Wat46

Wil83

Wis 67

Wit80

Yan84

Yas 83

D. Spears, H. Fischbeck, and T. Carlson, Phys. Rev. A9, 1603 (1974)

E. Takasugi, Phys. Lett. 149B, 372 (1984)

E. Tret'yakov, Izvest. Akad. Nauk SSSR. Ser. Fiz, 39, 583 (1975)

E. Tret'yakov, N. Myasoedov, A. Apalikov, V. Konyaev, V. Lyubimov, and E. Novikov, Izvest. Akad. Nauk SSSR. Ser. Fiz. 40, 2026 (1376)

R. Ulsh, H. Wellenstein, and R. Bonham, J. Chem. Phys. $\underline{60}$, 103 (1974)

J.-L. Vuilleumier, Proc. XIth Int. Conf. on Neutrino Physics and Astrophysics, p.94 (1984)

J. Walraven and I. Silvera, Rev. Sci. Instrum. 53, 1167 (1982)

R.D. Watts and D. Williams, Phys. Rev. 70, 640 (1946)

R. Williams and S. Koonin, Phys, Rev, C27, 1815 (1983)

H. Wise and B. Wood, Adv, in Atomic and Mol. Phys. 3, 291 (1967)

E. Witten, Phys. Lett. 91B, 81 (1980)

J. Yang, M.S. Turner, G. Steigman, D.N. Schramm and K.A. Olive, Astrophys. Journal 281, 493 (1984)

S. Yasumi, G. Rajasekaran, M. Ando, F. Ochiai, H. Ikeda, T. Ohta, and D. Stefan, Phys. Lett. 122B, 461 (1983) 
Acknowledgements :

This work was supported by the Department of Energy through a Los Alamos National Laboratory Institutionally Supported Research and Development grant. The experiment described herein was designed by and has been under the direction of T.J. Bowles and R.G.H. Robertson.

I would like to thank my advisors, Hamish Robertson at Los Alamos and Art McDonald at Princeton, for their help, encouragement, and advice, and for reading this work on such short notice. I would also like to thank my coworkers, Tom Bowles, John Wilkerson, Richard Martin, James Cohen, Tom Burritt, and Joe King for all their efforts and many profitable discussions. 